\title{
FLUE GAS DESULFURIZATION BY ROTATING BEDS
}

DOE/PC/79924--T6

DE93 004919

Final Technicall Report

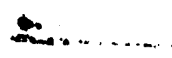

by

WW $4+100 h$

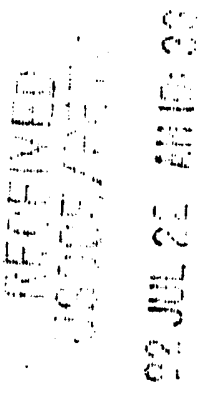

\author{
prepared for \\ US Department of Energy \\ Pittsburgh Energy Technology Center \\ Pittsburgh, Pennsylvania \\ DOE\#DE-FG22-87PC 79924
}

Nelson Gardner (PI)

Majid Keyvani

Aydin Coskundeniz

Department of Chemical Engineering

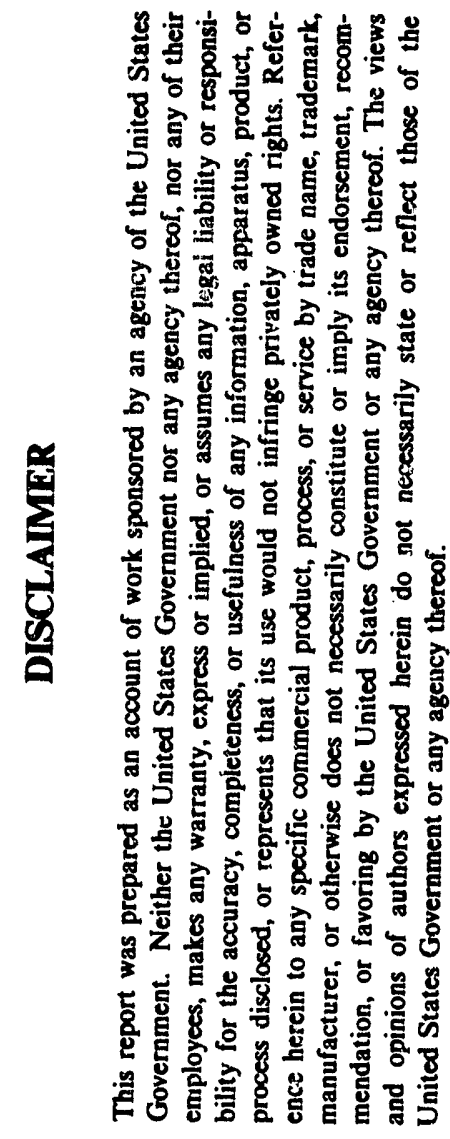

Case Westeru Reserve University

Cleveland, Ohio 44106

US/DOE putent clearance is not required prior to the publication of this document 


\section{PART I}

\section{OPERATING CHARACTERISTICS OF ROTATING FOAM METAL BEDS FOR FLUE GAS DESULFURIZATION}

BY

Majid Keyvani 
TABLE OF CONTENTS

1. INTRODUCTION

2. PREVIOUS WORK

2.1. Centrifugal Tray Absorber ................................................................

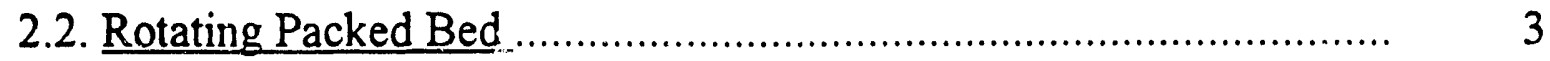

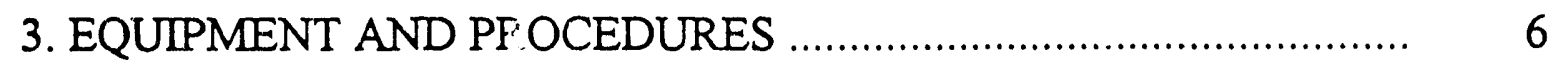

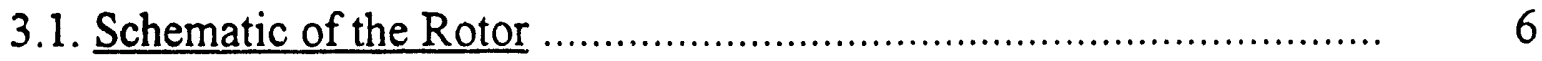

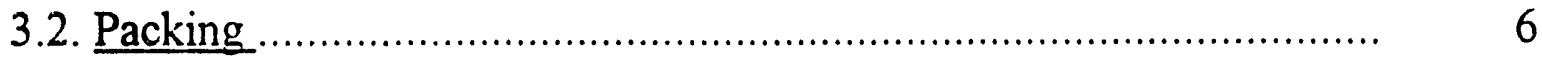

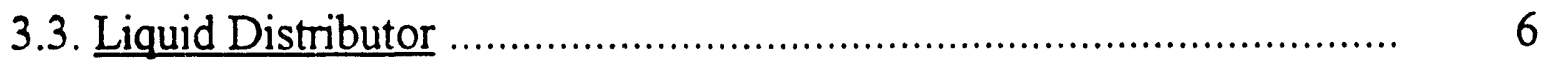

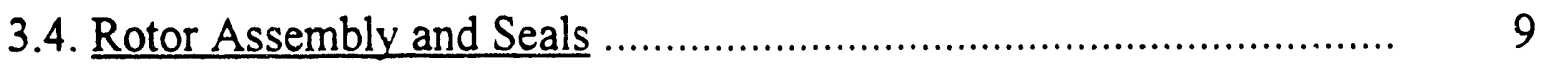

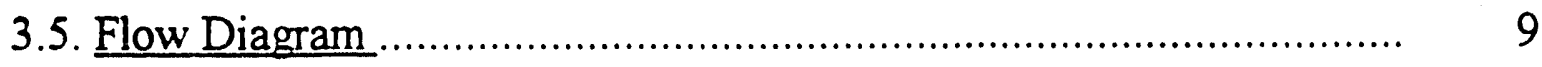

3.6. Measurements of Operating Parameters ...........................................

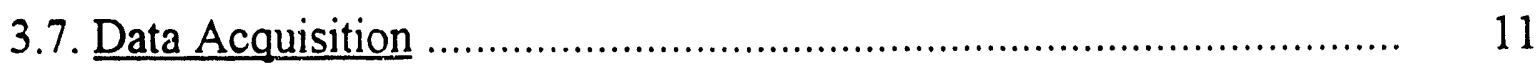

4. PRESSURE DROP CHARACTERISTICS OF ROTATING BEDS ..... 12

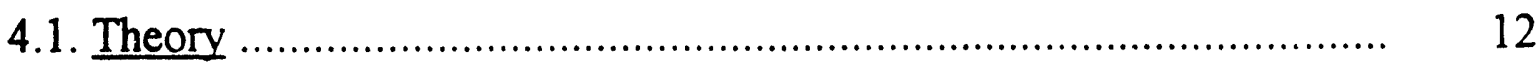

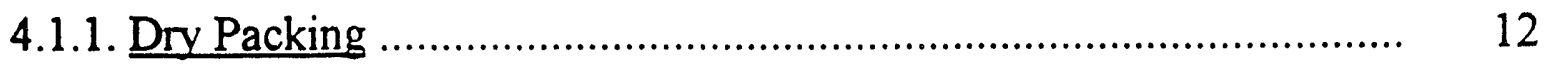

4.1.1.a. Pressure Drop Across the Bed .................................................. 12

4.1.1.b. Pressure Drop Between the Housing and Rotor .............................

4.1.2. Imigated Packing .................................................................... 16

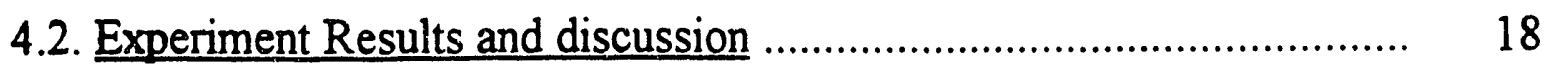


4.2.1. Typical Pressure Drop Observation ............................................. 18

4.2.2. Gravitational Effects ................................................................. 20

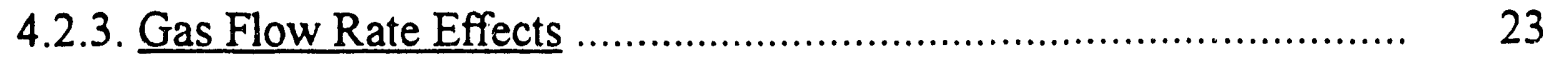

4.2.4. Liquid Flow Rate Effects …...................................................... 25

4.2.5. Effect of Packing Specific Surface Area ........................................ 25

4.3. Comparison with Model ........................................................... 29

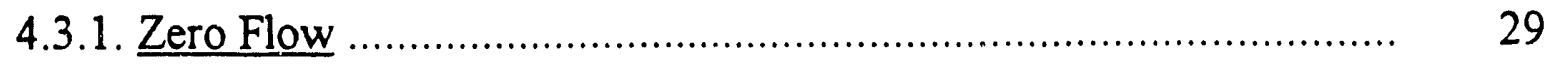

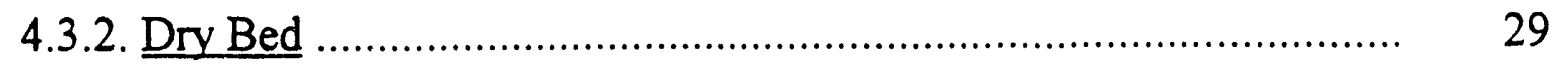

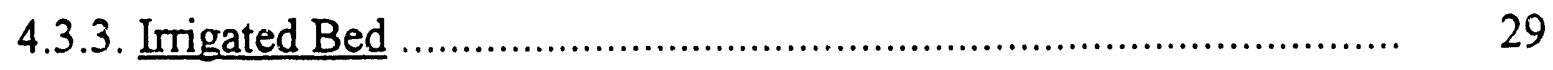

4.3.4. Comparison with Conventional Packed Bed ................................. 34

5. MASS TRANSFER CHARACTERISTICS OF ROTATING BEDS .... 37

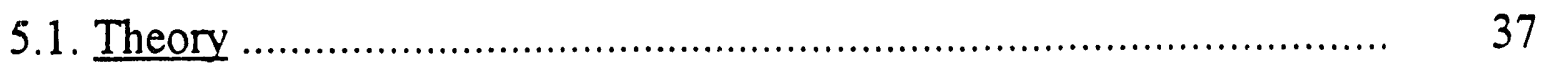

5.2. Experimental Results and Discussion ........................................ 45

5.2.1. Gravitational Effect .......................................................... 46

5.2.2. Effect of Packing Specific Surface Area ....................................... 46

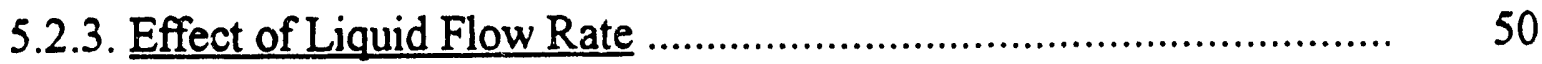

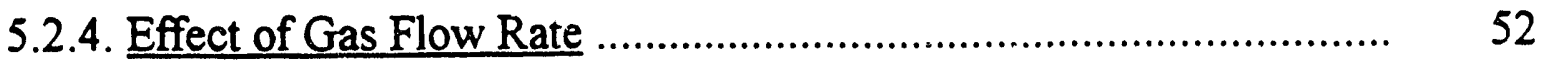

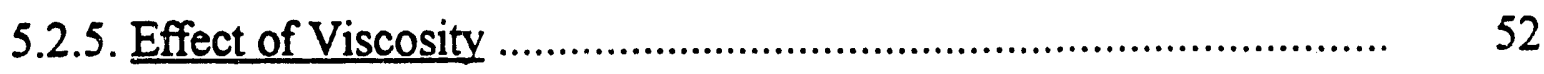

5.3. Comparison with Models ........................................................... 52 


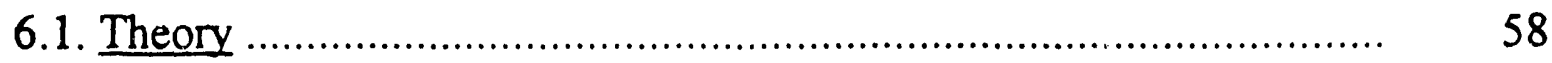

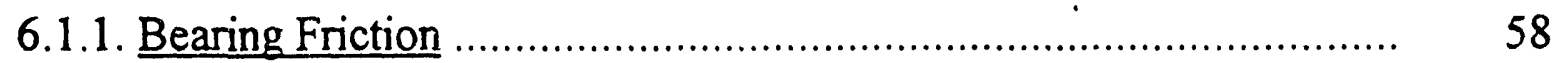

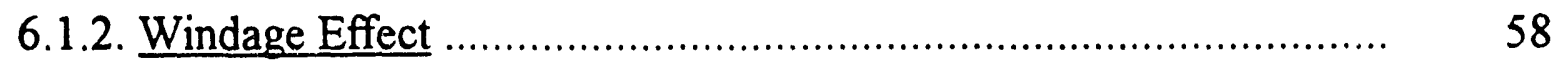

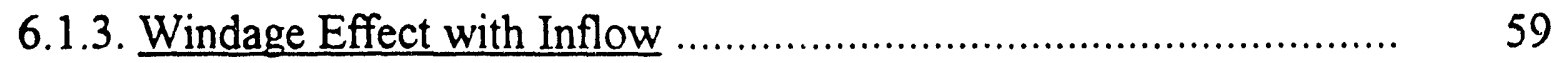

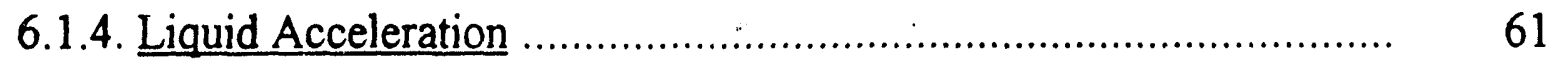

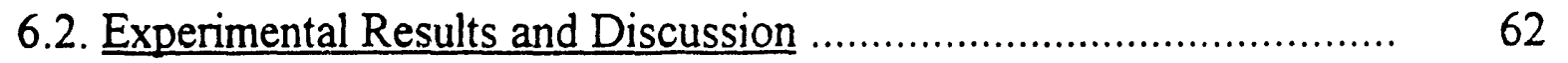

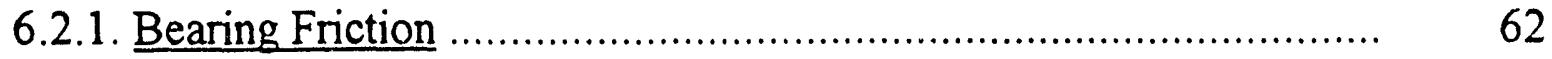

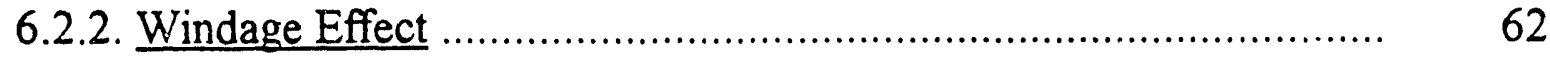

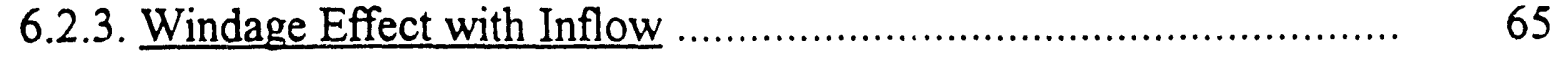

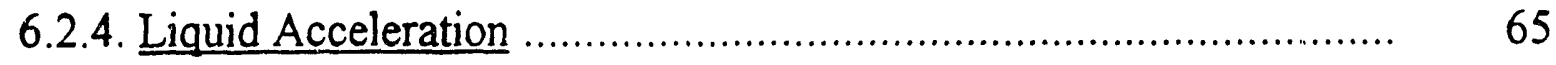

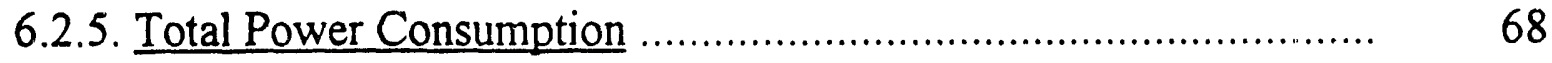

7. RESIDENCE TIME DISTRIBUTION .........................................

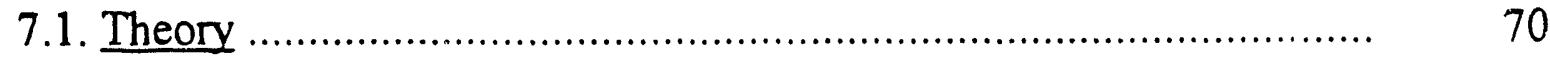

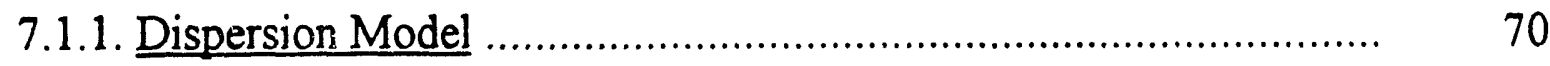

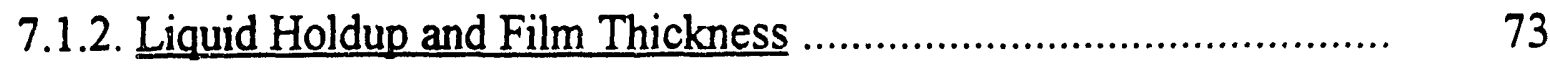

7.2. Experimental Results and Discussion ............................................

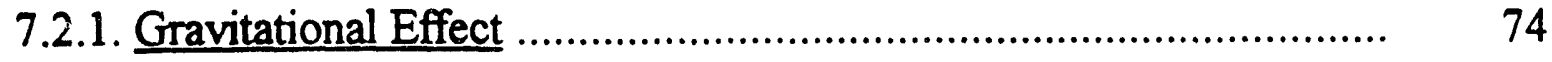

7.2.2. Liquid and Gas Flow Rate Effects ...............................................

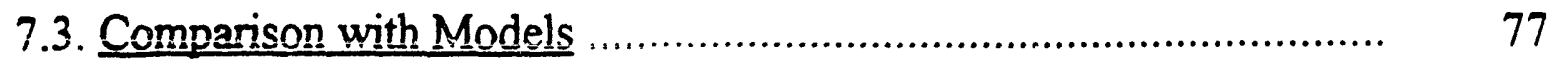


8. CONCLUSIONS AND RECOMMENDATIONS ............................ 82

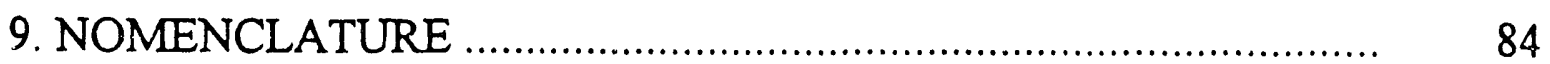

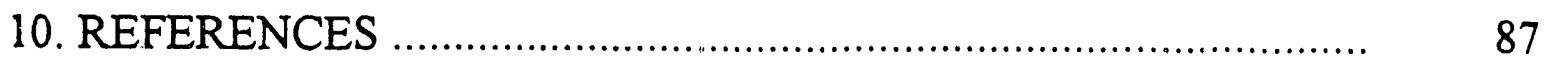

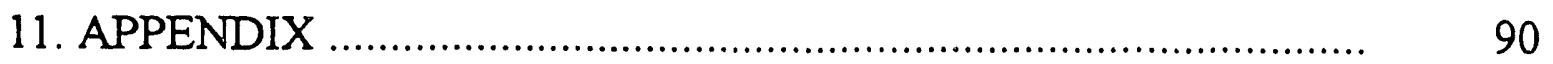

11.1. Stress Analysis for Aluminum Foam Metal Packing ....................... 90

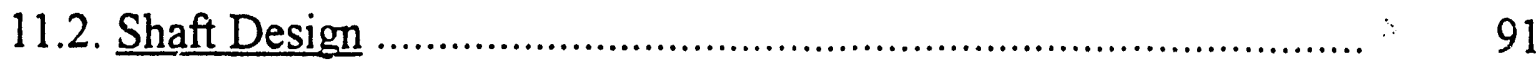

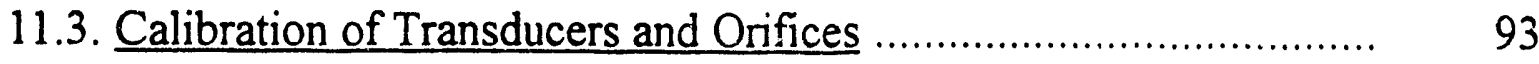

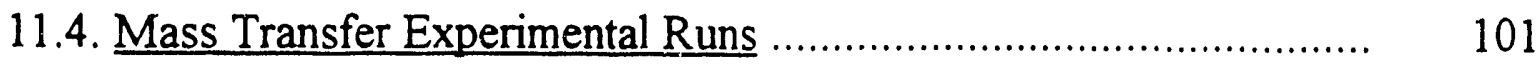

11.5. Residence Time Distribution Experimental Runs ........................... 106

11.6. Selected Coordinate System for the Control Volume ...................... 128 


\section{OPERATING CHARACTERISTICS OF ROTATING FOAM METAL BEDS FOR FLUE GAS DESULFURIZATION}

\section{INTRODUCTION}

Flue gas desulfurization (FGD) is an important problem, particularly considering the utilization of the high-sulfur coals for power generation. The economical separation of sulfur dioxide from flue gas is difficult, as the volume of gas to be treated is large, about 3000 actual cubic feet per minute per megawatt generating capacity, and the concentration of sulfur dioxide in the flue gas is small, on the order of $0.5 \%$.

Absorption processes, the scrubbing of sulfur dioxide by counter-current contacting of the flue gas and solvent in a packed tower, is one approach. Substantial improvements in scrubber performance have been achieved through superior packing design, but that technology is now mature, and further substantial gains in performance are unlikely. The perception has been that the major improvements in the economics of FGD would most likely be forthcoming in novel process ideas, careful selection of solvents, and care in the conversation of energy.

Process intensification, the use of high gravitational, electric acoustic, or magnetic fields to enhance reaction and separation processes, is a very interesting and promising research and development area. In particular, heat and mass transfer can be intensified by carrying out processes in high gravitational fields. Imperial Chemical Industries (ICI) pioneered the development of rotating packed beds, which are one to two orders of magnitude smaller in volume than conventional packed columns. Heights of theoretical plates are much reduced, typically from about two feet to four feet in conventional column, operating at $1 \mathrm{~g}$ t to one-half to one inch in rotating packed beds, operating at 300 g's. Benefits include:

- much smaller equipment and supporting structures

- much reduced solvent inventory 
- shorter start-up and shut-down times

- faster response with improved process control

- reduced pressure drop

The benefit of much reduced solvent inventory is particularly important, as there is the possibility of using solvents that hitherto have been considered too expensive for use in FGD scrubbers. With rotating packed beds, there is also the possibility of substantial gains in scrubber performance and a possible breakthrough in the economics of flue gas desulfurization. To date, there is little in the literature about the operating characteristics of rotating beds, particularly high performance foam metal beds.

This work is concerned broadly with the operating characteristics of rotating beds. The objectives of this work are to 1) characterize the pressure drop in rotating bed, 2) investigate the effect of gravitational acceleration on volumetric mass transfer coefficients of $\mathrm{SO}_{2}$ absorption, 3) determine the residence time distribution of the liquid phase in rotating beds, 4 ) investigate the required power consumption of rotating beds. 


\section{PREVIOUS WORK:}

\subsection{Centrifugal Tray Absorbers:}

The concept of contacting liquids and vapors in a centrifugal field was reported in early studies of Podbielniak $[1,2]$. The centrifugal absorber consisted of perforated cylindrical sieve trays for gas-liquid contacting. The perforations were dimpled inward to provide weirs for retaining some of the liquid. The perforations were off-set axially from tray to tray to prevent any direct short circuiting. This device was later improved by Todd $[3,4]$ to provide more effective contacting for the phases, and to increase the stage capacity by utilizing a number of units operating on a vertical shaft. Similar work was reported by Chambers and Walls [5]: their centrifugal absorber consisted of a set of rings attached to :te rotating plate to generate climbing liquid films and sprays repeatedly.

\subsection{Rotating Packed Beds:}

Vivian et al [6] studied the effect of gravitational field on gas absorption in a packed column by mounting a cylindrical packed bed on the horizontal arm of a large centrifuge. The gravitational force was varied from 1 to 6.4 times the gravity force. For their system configuration, the liquid phase volumetric mass transfer coefficient varies with the gravitational force to the 0.41 to 0.48 power.

In the late 1970's, there appeared in the European and U.S. patents literatures a series of patents assigned to Imperial Chemical Industries (ICI London) [7-10], introducing the HIGEE packed bed rotor for absorption and distillation applications. The HIGEE packed bed was operated at mean acceleration fields of 200 to $500 \mathrm{~g}$, and the reported volumetric mass transfer 
coefficients were up to 100 times larger than the values obtained in conventional packed columns. The reported volumetric mass transfer coefficients varied with the gravitational force to the 0.14 to 0.54 powers. $\mathrm{ICI}$, in spite of extensive tests, published very little information. Glitch Inc. (Dallas), which has acquired worldwide marketing rights, also reported a test history of HIGEE with various systems at a wide range of process conditions and physical properties [11]. Glitch Inc. already installed a unit two years ago for the U.S. Coast Guard at a facility near Traverse City, Michigan, ic air-strip aviation fuel from contaminated ground water, and field tests are underway for an undisclosed natural-gas producer in the U.S. [12]. However, no publications are cited in the open literature about the results of the tests.

Recently, Dudukovic et al reported theoretical and experimental work on rotating packed beds $[13,14,15]$. They reported separately the effect of rotational speed on gas-liquid interfacial area and liquid side mass transfer coefficient to explain the overall enhancement of volumetric mass transfer coefficients in rotating packed beds. Their findings show that the volumetric mass transfer coefficient increases with the rotational speed as a fractional power of 0.61 to 0.59 which is in agreement with the data of Vivian et al and within the range estimated by ICI. They showed that the effective surface area increases with the body force with 0.14 to 0.28 power (contribution of effective surface area to the enhanced volumetric mass transfer coefficient). Their theoretical framework was based on film flow on rotating blades and rotating disks. Their flooding tests with air-water system showed that the Sherwood correlation for the dumped rings is 
conservaive by $40-70 \%$. The bed in this study was $3 \mathrm{~mm}$ glass beads which had a specific surface area of $120 \mathrm{~m}^{2} / \mathrm{m}^{3}$ and a porosity of 0.4 .

In all the above work, no attempt was made to model the pressure drop characteristics of the rotating pacied bed, nor is there any information available about residence time distribution on rotating packed beds. No work has been reported in the literature on power requirement of rotating packed beds. 


\section{EQUIPMENT and PROCEDURE:}

\subsection{Schematic of the Rotor:}

Figure 1 shows a simplified schematic of the rotating bed. The liquid enters from the inner pipe of the stationary double pipe (2), is sprayed by the distributor (1) on the inside edge of the bed, and then is thrown out into the housing (3) by the centrifugal force. The gas enters the stationary hrusing and, due to the imposed pressure gradient, flows countercurrent to the liquid hrough the rotating bed. After leaving the bed, the gas exits through the double pipe (4). The liquid collects in the bottom of the housing wall and forms a liquid seal (5) in a trap.

\subsection{Packing:}

The packing for the rotating bed is a one piece torus-shaped unit (6), made of an aluminum foam metal. It is dimensionally stable in the high gravitational fields. The outside diameter is $45.7 \mathrm{~cm}$, the inside diameter is $25.4 \mathrm{~cm}$, and the thickness is $4.4 \mathrm{~cm}$. Three different beds were studied. The beds'specifications are presented in Table 1. Our stress analysis indicates the beds can be operated from 600 to $2000 \mathrm{rpm}$, giving a gravitational field of 100 to 1000 times gravity (see the Appendix).

\subsection{Liquid Distributor:}

The liquid distributor is a manifold consizting of twelve distributing tubes. The division of the liquid stream into parts by means of this type of manifold is uneven because it is not possible to keep the liquid pressure perfectly constant inside the main channel [16]. However, for the above specifications, the difference 


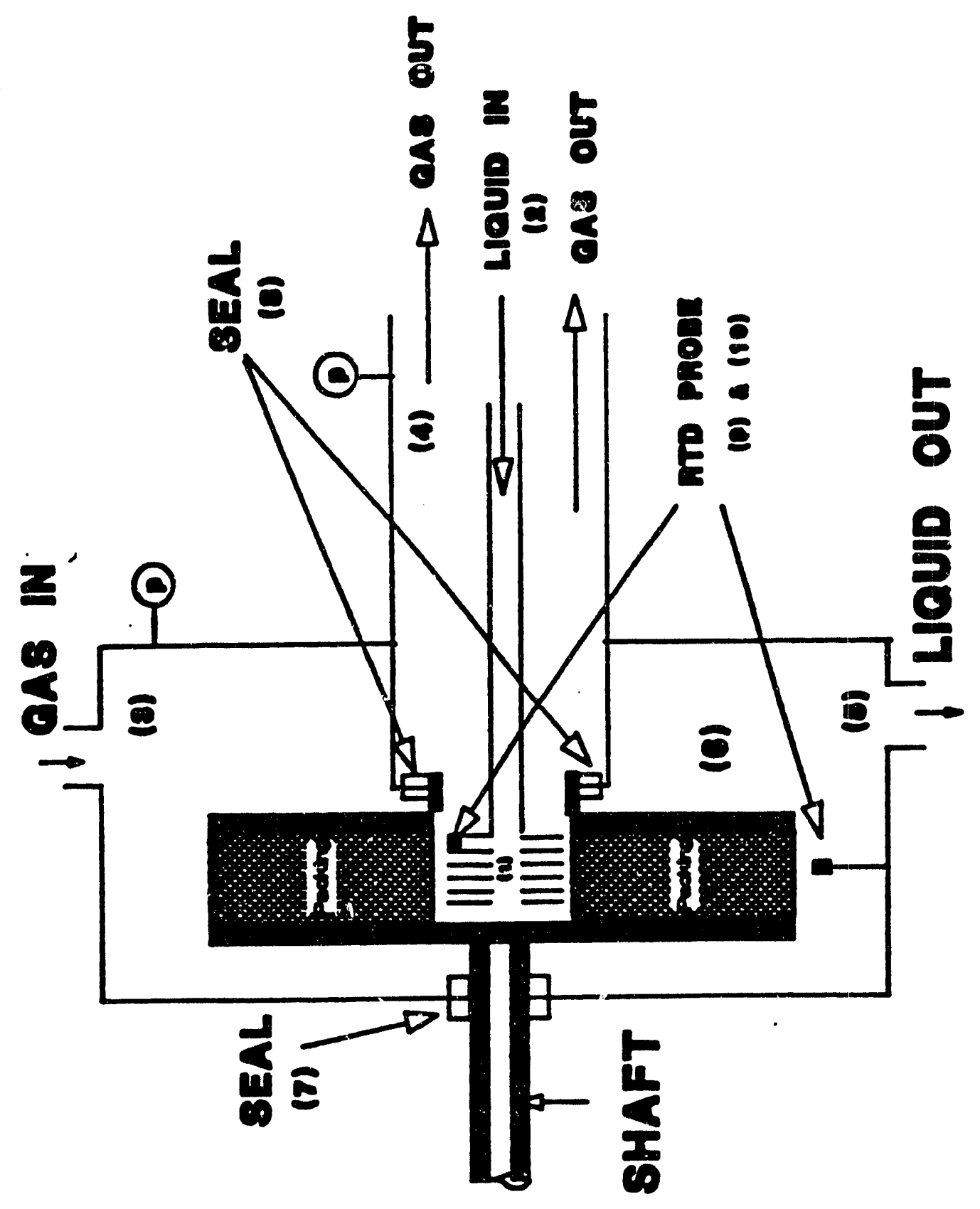

Figure 1. Schematic of Rotating Bed 
TABLE 1. PACINIG SPDCIFTCATION

\begin{tabular}{|c|c|c|c|}
\hline $\begin{array}{l}\text { Specific Surface } \\
\text { Area }\left(\mathrm{m}^{2} / \mathrm{m}^{3}\right)\end{array}$ & Porosity & of Pores/cm & Pore Size \\
\hline 656 & 0.92 & 4 & 0.4 \\
\hline 1476 & 0.92 & 8 & 0.2 \\
\hline 2952 & 0.92 & 16 & 0.1 \\
\hline
\end{tabular}


in the rate of the flow between two extreme ports is less than $5 \%$. This manifold provided adequate liquid distribution and reduced the chance of the liquid entrainment in the inner edge of the rotor where the llooding is most probable, since the cenirifugal acceleration is lowest and the gas velocity highest at this point.

\subsection{Rotor Assembly and Seals:}

The rotor assembly is mounted on a $5.1 \mathrm{~cm}$ diameter shaft, supported by two bearings, in a cantilevered design. A variable speed $5 \mathrm{hp} \mathrm{AC}$ motor with a belt drives the rotor assembly.

Seals are required in two locations in the rotor assembly: the shaft $(\tau)$, and the double pipe assembly for the gas exit (8). Inexpensive silicone rubber radial lip seals were used in both locations.

\subsection{Flow Diagram:}

Figure 2 shows the flow diagram of the general experimental apparatus. The feed and recycle pumps can supply up to $45 \mathrm{~kg} / \mathrm{sec}-\mathrm{m}^{2}$ for this packing ( $30 \mathrm{gpm}$ ). The compressor can deliver up to $2.5 \mathrm{~kg} / \mathrm{sec}-\mathrm{m}^{2}$ air for this packing (120 acfm). The air can be heated or it can enter the rotor at room temperature.

\subsection{Measurements of Operating Parameters:}

Both the liquid and vapor flowrates were determined by measuring the pressure drop across restriction orifices (RO). Differential pressure transducers were used to measure the pressure drop across the orifices. The pressure drops across the bed were measured by placing the probes for the transducers at the inlet and outlet of the rotor as shown in Figure 1. 


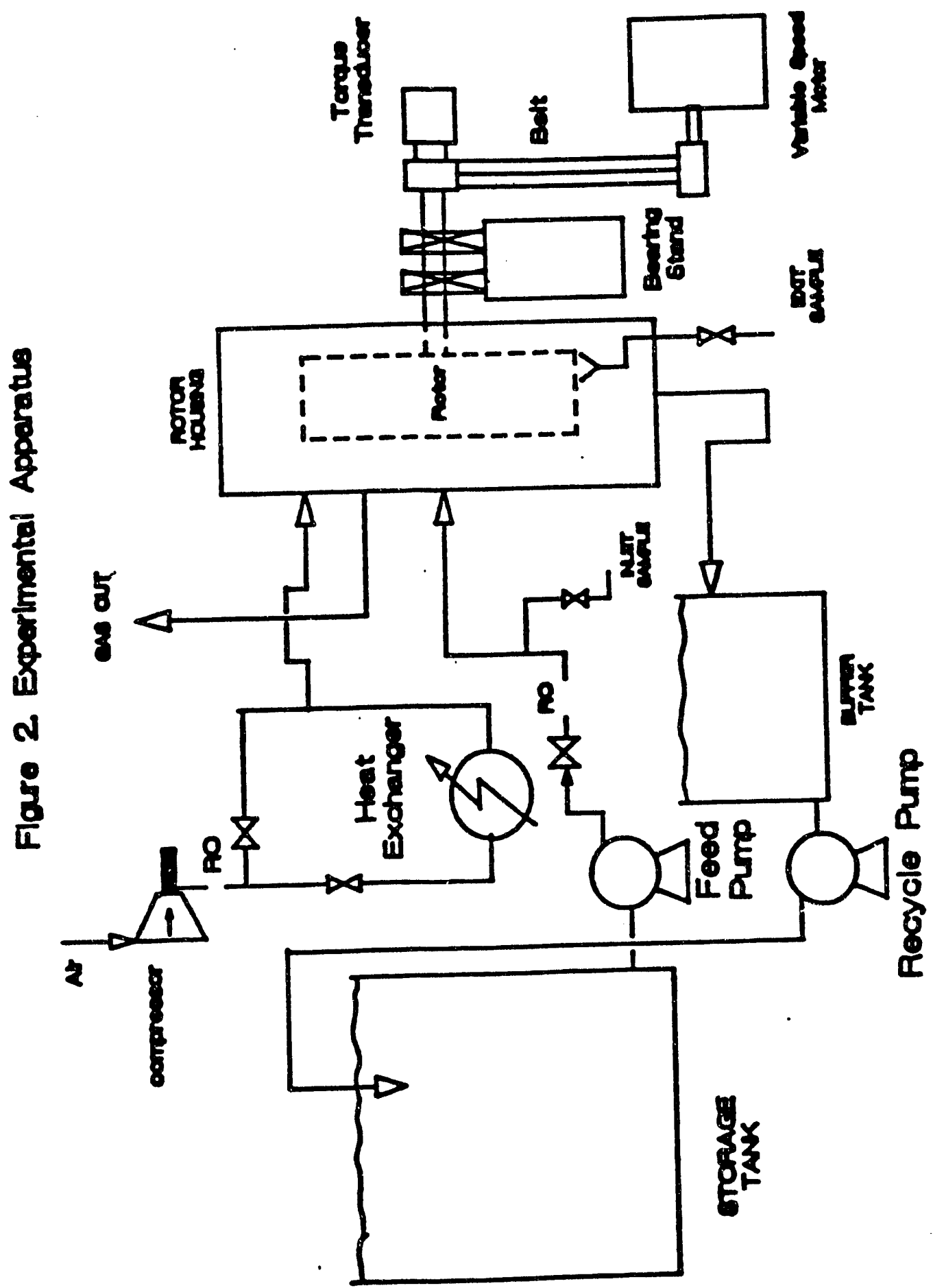


The torque and rotational speed measurements were made to determine the power required to rotate the beds. A sprocket/pulley torque transducer was connected to the end of the drive shaft to measure the required torque for the rotating bed. A magnetic pick-up tachometer was used to measure the speed of rotation.

For residence time distribution experiments (RTD), concentrated $\mathrm{NaNO}_{3}$ solutions were prepared and charged to a tracer inlet loop. By a simultaneous half turn of two shut-off valves, the tracer was introduced into the liquid stream inlet. The liquid passes the RTD sensors at the inlet (6) and outlet (7) of the rotor. The electrical conductivity inlet sensor consisted of two $0.158 \mathrm{~cm}\left(1 / 16^{\prime \prime}\right)$ stainless steel rods mounted perpendicularly and 180 degrees apart on the body of a teflon tube. The teflon tube serves as one of the distributing tubes for the liquid distributor. The outlet sensor consisted of two $0.158 \mathrm{~cm}$ stainless steel rods (1/16" rods) mounted on a teflon trough which is placed very close to the rotor outlet. The purpose of the trough design is to catch the spray from the rotor.

Carbon dioxide concentrations in the liquid were determined by a specific ion electrode. Carbon dioxide concentration in the gas phase were detcrmined by gas chromatography.

\subsection{Data Acquisition:}

All the flow, pressure, temperature, torque, and rotational speed transducers are interfaced to a computer. The data for the RTD experiments was taken by a digital oscilloscope. 


\section{PRESSURE UROP CHARACTERISTICS OF ROTATING BEDS}

\subsection{THEORY:}

\subsubsection{Dry Packing:}

The total pressure drop in rotating packed beds is the sum of pressure drops from two regions: the pressure drop between the stationary housing and the spinning rotor, $\Delta \mathrm{P}_{0}$, and the pressure drop inside of the rotor (across the bed), $\Delta \mathrm{P}_{\mathrm{i}}$. Each region will be analyzed.

\subsubsection{Pressure Drop Acrass the Bed:}

If the pressure drop through the bed is not large, the gas phase can be assumed incompressible. Assuming that the velocity in the axial direction is zero, and the angular velocity does not vary in the tangential direction, then the continuity equation in a cylindrical coordinate for the gas phase, at steady state, may be written as:

$$
\frac{1}{r} \frac{\partial}{\partial t}(\epsilon \mathrm{r} V)=0
$$

Here $V$ is the velocity in radial direction, $r$ is the radial distance, and $\epsilon$ is the packing porosity. See the Appendix for the selected coordinate system for a control volume.

A general momentum balance can be written as follows:

\{rate of momentum accumulation $\}=\{$ rate of momentum in $\}-$ \{rate of momentum out $\}+$ sum of forces acting on system $\}$ 
The radial momentum balance, at steady state, may be expressed as:

\{change in momentum $\}=$ sum of pressure, drag, and centrifugal forces $\}$, while in the targential direction ( $\theta$ direction) the momentum balance may be written as: \{change in momentum $\}=\{$ sum of drag and coriolis forces $\}$.

The equations of motion bave the following forms:

$$
\begin{array}{ll}
-\rho_{\mathrm{g}} \mathrm{V} \frac{\mathrm{dV}}{\mathrm{dr}}=\frac{\mathrm{dP}}{\mathrm{dr}}+\rho_{\mathrm{g}} \frac{\mathrm{U}^{2}}{\mathrm{r}}+\frac{\text { Drag Force }}{\text { unit volume }} & \text { r-direction } \\
-\rho_{\mathrm{g}} \mathrm{V} \frac{\mathrm{dU}}{\mathrm{dr}}=\rho_{\mathrm{g}} \frac{\mathrm{UV}}{\mathrm{r}}+\frac{\text { Drag Force }}{\text { unit volume }} & \text { O-direction }
\end{array}
$$

Here $U$ is the tangential velocity, $P$ is the pressure, and $\rho_{\mathrm{g}}$ is the gas density.

To further simplify the model, a solid body rotation in the bed is considered and the coriolis force is ignored (an assumption that may be verified later). Assuming that the drag force exerted on the unit volume of gas by viscous transfer in the bed has the form of Ergun correlation (or similar available models such as the Carman-Kozeny or Morton model), equations (2) and (3) reduce to:

$-\rho_{\mathrm{g}} \frac{\bar{V}}{\bar{E}^{2}} \frac{d \bar{V}}{d r}=\frac{d P}{d r}+\rho_{g} \omega^{2} r+\rho_{g} A \bar{V}+\rho_{g} B \bar{V}^{2}$

Here $\bar{V}$ is the local superficial gas velocity, related to interstitial gas velocity by $\bar{V}=V_{\epsilon}, \omega$ is the rotational speed, and $A$ and $B$ are Ergun correlation coefficients which depend on gas physical properties and the packing specifications and defined as follows: 
For Ergun's model: $A=150(1-\epsilon) \mu_{g} / \epsilon^{3}\left(\varphi_{s} d_{p}\right)^{2}, B=1.75(1-\epsilon) \rho_{g} / \epsilon^{2} \varphi_{s} d_{p}$ For Morton's model: $A=8.5 \mu_{g} a_{p} / \epsilon^{3}, B=a_{p} \rho_{g}(R e)^{-1} / \epsilon^{3}$

Here $a_{p}$ is the specific surface area of the packing, $d_{p}$ is the packing diameter, $\varphi_{s}$ is the sphericity of the packing particles, and Re is the gas Reynold's number based on the specific surface area. In general, these models are written in terms of the slip velocity of the two phases. However, since the superficial solid velocity is zero, only the gas velocity appears in the equation (we will see later that this is not the case when the bed is irrigated by liquid).

Equation (4) may be integrated across the entire bed to obtain the pressure drop inside of the rotor, $\Delta \mathrm{P}_{\mathrm{i}}$, for a dry bed.

\subsection{1.b. Pressure Drop Between the Housing and Rotor:}

Outside of the rotor, we have a flow situation similar to flow between two disks, one spinning and one stationary, with the inflow. There have been numerous studies of this type of flow in the literature [17-21]. The basic flow configuration is as follows: the rotating disk imparts rotation to the fluid, leading to a distribution of angular velocity from the rotating disk value at the disk to zero at the stationary bousing wall. The drag forces act on the fluid in the direction of rotation at the rotating disk and in the opposite direction at the wall surface. The pressure gradient is generated centrifugally, in the gap between the disk and the wall, and its value depends on the effective average angular velocity of the flow 
field. The pressure tends to be a function of radius only and independent of axial position in the gap. The angular velocity of the fluid near the rotating disk is above the effective average, and hence the fluid near the rotating disk is thrown radially outward. On the other hand, fluid close to the stationary housing has smaller angular velocity than the effective average so that it flows radially inward. The effect of inflow due to leakage (in the rotating packed bed the leakage is the total gas flow rate) is to increase the radial pressure gradient. This increase in pressure gradient can be explained in terms of the rotation effect of inflow. The fluid tends to rotate faster due to inflow and so generates larger pressure gradient.

A momentum integral method is used to analyze the pressure gradient between the rotating disk and the stationary bousing. The governing equations are the same as equations (1) to (3). The difference is that the simplifications made in evaluating $\Delta \mathrm{P}_{\mathrm{i}}$, ignoring the coriolis force and the assumption of solid body rotation, are not valid here. A distribution of tangential velocity exists which can be obtained by solving equation (3). The drag forces are the radial and tangential friction stresses on the wall and the rotating disk. These forces are deduced from work performed by Von. F. Shultz-Grunow [20] and are defined as follows:

$\tau_{\mathrm{rw}}$ stress in radial direction at wall $=\rho_{\mathrm{r}} \rho_{\mathrm{g}} \frac{U^{2}}{2}$

$\tau_{r d}$ stress in radial direction at rotating disk $=\rho_{\mathrm{r}} \rho_{\mathrm{g}} \frac{(\mathrm{r} \omega-U)^{2}}{2}$

$\tau_{\text {tw }}$ stress in tangential direction at wall $=f_{\imath} \rho_{g} \frac{U^{2}}{2}$

$\tau_{\text {Ld }}$ stress in tangential direction at rotating disk= $\int_{\mathfrak{l}} \rho_{g} \frac{(r \omega-U)^{2}}{2}$ 
where $f_{r}$ and $f_{t}$ are the friction coefficients that take the following expressions for the turbulent flow:

$$
\begin{aligned}
& f_{r}=\frac{0.0391}{\left(\rho_{g} \omega r_{0}^{2} / \mu_{g}\right)^{0.2}} \times \frac{1}{\left(1-r / r_{0}\right)^{0.4}} \\
& f_{t}=\frac{0.0996}{\left(\rho_{g} \omega r_{0}^{2} / \mu_{g}\right)^{0.2}} \times \frac{1}{\left(1-r / r_{0}\right)^{0.4}}
\end{aligned}
$$

Here $\mu_{\mathrm{g}}$ is the gas viscosity, and $r_{0}$ is the disk outside radius. After substituting for $f_{r}$ and $f_{i}$ from equations (5) and (6), equations (2) and (3) can be solved simultaneously to evaluate the pressure gradient in the gap between the rotating disk and the stationary wall, $\Delta \mathrm{P}_{0}$. The equations were solved numerically and a $4^{\text {th }}$ order Runge-Kutta method was employed to approximate the integrations.

\subsubsection{Irrigated Packing:}

\subsection{2.a Pressure Drop Across the Bed:}

To extend the proposed model for dry packing to the irrigated packing equation (4) will be modified to account for the liquid presence. The velocity terms related to Ergun formula will be written in terms of the slip velocity of the gas and liquid phases:

$$
\frac{d P}{d I}=A\left(\bar{V}-V_{i}\right)+B\left(\bar{V}-V_{i}\right)^{2}+\rho_{g} \omega^{2} t+\rho_{g} \frac{d \bar{V}}{d r}
$$

Here $\mathrm{V}_{\mathrm{i}}$ is the interfacial velocity. 
The equation of motion for the liquid steam, assuming laminar and fully developed flow within the short distance that both phases are brought into contact, may be written as follows:

$$
\mu_{1} \frac{d^{2} V_{1}}{d z^{2}}=-\frac{d P}{d r}+\rho_{1} w^{2} r
$$

Here $V_{1}$ is the liquid phase velocity, $\mu_{1}$ is the liquid viscosity, $\rho_{1}$ is the liquid density, and $z$ is the distance in direction perpendicular to the radial direction. The above equation can be solved based on the boundary conditions:

$$
\begin{aligned}
& V_{1}=v_{i} @ z=\delta \\
& v_{1}=0 @ z=0
\end{aligned}
$$

$\delta$ is the liquid film thickness. The solution, after integrating over the liquid film thickness, may be written in terms of the liquid flowrate as follows:

$$
Q=\left(\rho_{1} \omega^{2} r-\frac{d P}{d r}\right) \frac{h \delta^{3}}{12 \mu_{1}}+\frac{h V_{i} \delta}{2}
$$

where $Q$ is the liquid flowrate, and $h$ is the axial height of the packing.

Equations (7) and (9) are coupled through $d P / d r, V_{i}$, and $\delta$ terms.

Therefore, one more equation is needed to solve for the above unknowns and that arises from the boundary condition indivating equal stresses at the gas and liquid interface: 


$$
\tau_{g}=\tau_{1} \bigcirc 2 . \delta
$$

Here $\tau_{\mathbf{g}}$ and $\tau_{1}$ are the gas and liquid shear stresses, respectively.

Solving the equations by using the above boundary condition is excessively involved and complicated. Instead, we try to estimate the liquid film thickness by using either the asymptotic solution for liquid film on a rotating disk [13] or liquid film flow on an inclined surface [22] after substituting centrifugal acceleration for the gravity (it is shown later that the film thickness can be estimated for a rotating bed by carrying out the Residence Time Distribution (RTD) experiments).

For given film thickness, gas and liquid flowrates, speed of the rotation, and other liquid and gas physical properties, equations (7) and (9) can be solved to evaluate the pressure drop across the rotating bed. A $4^{\text {th }}$ order Runge Kutta method was employed to solve the equations numerically.

\subsection{2.b. Pressure Drop Between the Housing and Rotor:}

The above analysis for the irrigated packing gives the pressure drop across the bed. It is assumed that the pressure drop between the the rotating disk and the stationary housing is not affected by the presence of the liquid; therefore, the same analysis as dry packing is applied for the irrigated packing for the outside of the rotor.

\subsection{EXPERIMENTAL RESULTS \& DISCUSSIONS:}

\subsubsection{Typical Pressure Drop Observation:}

A peculiar phenomenon is observed in pressure drop measurements of rotating beds. Figure 3 shows a typical plot of pressure drop against time. When 


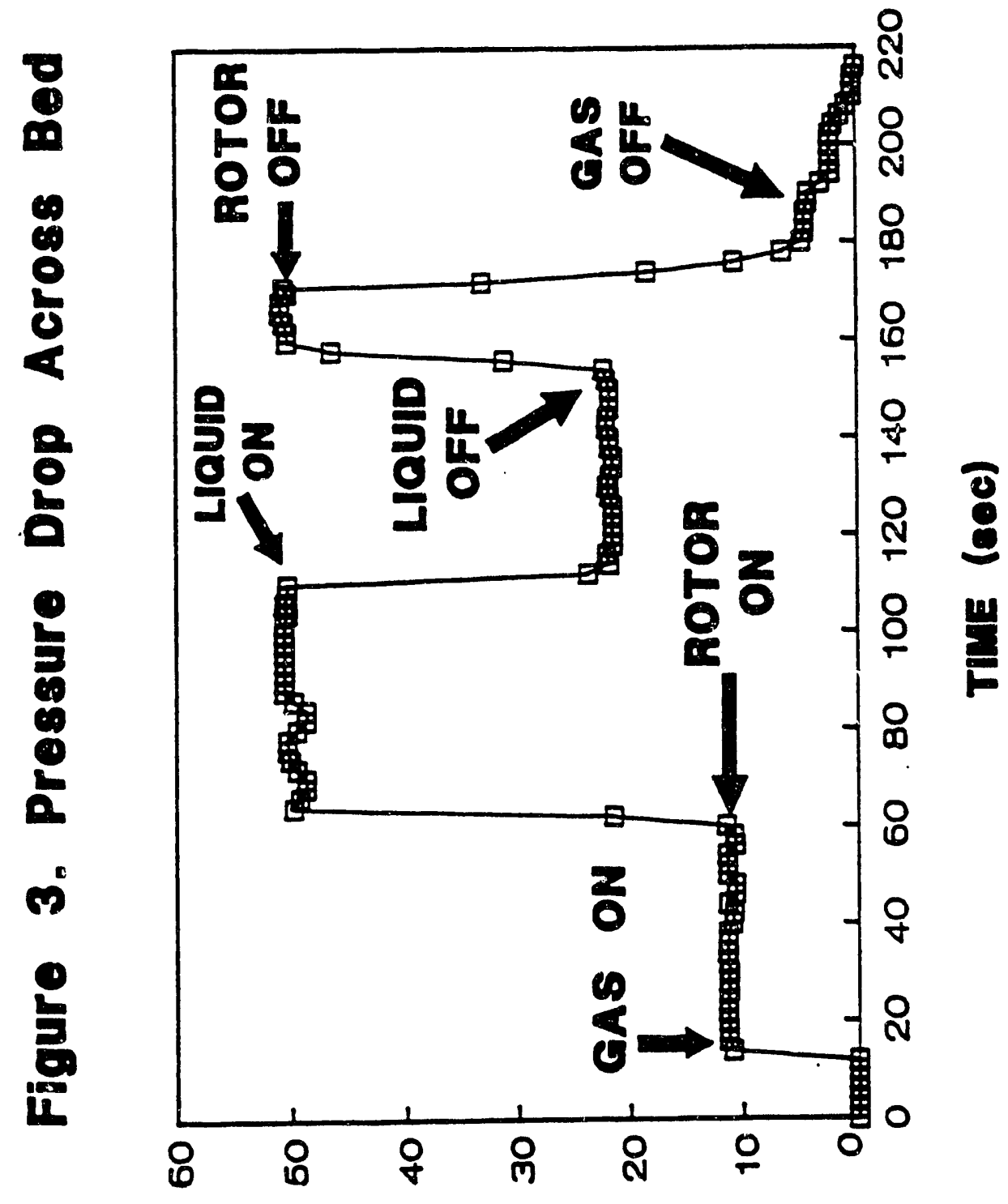

pored dong anewed 
the rotor is stationary and the gas is turned on, the pressure drop is $11 \mathrm{~Pa} / \mathrm{cm}$ of bed. When the rotor is turned on, the pressure drop increases to $50 \mathrm{~Pa} / \mathrm{cm}$ of bed. When the liquid is turned on, countercurrent to the gas flow, the pressure drop decreases by more than a factor of 2, to $22 \mathrm{~Pa} / \mathrm{cm}$ of bed. For this experiment, a gas flowsate of $1.0 \mathrm{~kg} / \mathrm{sec}-\mathrm{m}^{2}$ and a liquid flowrate of $36 \mathrm{~kg} / \mathrm{sec}-\mathrm{m}^{2}$ were user. Packing surface area was $1476 \mathrm{~m}^{2} / \mathrm{m}^{3}$, and the packed bed was rotating at 600 rpm.

To understand this phenomenon, various experiments were performed to isolate the influence of different operating parameters.

\subsubsection{Gravitational Effects (Constant Liquid and Gas Flow):}

Experiments were carried out to measure the pressure drop at various acceleration fields (varying speed of rotation). The liquid and gas flowrates were held constant at $36 \mathrm{~kg} / \mathrm{sec} \mathrm{m}^{2}$ and $1 \mathrm{~kg} / \mathrm{sec} \mathrm{m}^{2}$ respectively. The packing with the specific surface area of $1476 \mathrm{~m}^{2} / \mathrm{m}^{3}$ was used. The pressure drops were measured for the following situations:i) zero flow (gas off-liquid off), ii)liquid off-gas on, iii)liquid on-gas off, and iv) liquid on-gas on. Representative data are presented in Table 2. and are plotted in Figure 4.

At zero flow a radial pressure gradient develops because of the rotation of the air both inside the rotor and between the rotor and the stationary housing. This is associated with the pressure required to overcome the centrifugal head set due to the rotation. The pressure drop iscieases as the gas flow rate is turned on (gas on-liquid off). This increase results mainly from the drag (frictional pressure drop) and inertia effects. The measured pressure drops for the case of liquid-on 
TABLE 2. RESULTS OF PRESSORE DROP IT VIRYTIG ROTATIONAL SPEED

\begin{tabular}{|c|c|c|}
\hline $\begin{array}{l}\text { State of Experimental } \\
\text { Conditions }\end{array}$ & $\begin{array}{l}\text { Speed of Rotation } \\
(\mathrm{rp})\end{array}$ & $\begin{array}{c}\text { Pressure Drop } \\
(\mathbb{( T , / \mathrm { cm } )}\end{array}$ \\
\hline $\begin{array}{l}\text { LIQUTD OFF } \\
\text { GAS OFF }\end{array}$ & $\begin{array}{r}400 \\
600 \\
679 \\
797 \\
853 \\
944 \\
1025 \\
1182 \\
1253\end{array}$ & $\begin{array}{l}10.3 \\
17.0 \\
22.1 \\
29.4 \\
34.8 \\
: 2.3 \\
49.2 \\
64.6 \\
72.0\end{array}$ \\
\hline $\begin{array}{l}\text { LIQUTD OFF } \\
\text { GAS ON } \\
\left(1.0 \mathrm{~kg} / \mathrm{sec} \mathrm{m}^{2}\right)\end{array}$ & $\begin{array}{r}400 \\
600 \\
681 \\
790 \\
855 \\
947 \\
1047 \\
1136 \\
1203\end{array}$ & $\begin{array}{r}50.9 \\
69.5 \\
86.2 \\
109.9 \\
127.3 \\
147.4 \\
174.1 \\
189.3 \\
221.1\end{array}$ \\
\hline $\begin{array}{l}\text { LIQUTD ON } \\
\left(36 \mathrm{~kg} / \mathrm{sec}^{2}\right) \\
\text { GAS OFF }\end{array}$ & $\begin{array}{r}400 \\
600 \\
652 \\
740 \\
830 \\
937 \\
1039 \\
1159 \\
1269\end{array}$ & $\begin{array}{r}9.9 \\
16.7 \\
17.4 \\
19.6 \\
26.6 \\
35.8 \\
45.8 \\
54.1 \\
65.5\end{array}$ \\
\hline $\begin{array}{l}\text { LIQUTD ON } \\
\left(36 \mathrm{~kg} / \mathrm{sec}^{2}\right) \\
\text { GAS ON } \\
\left(1.0 \mathrm{~kg} / \mathrm{sec}^{2}\right)\end{array}$ & $\begin{array}{r}400 \\
600 \\
700 \\
840 \\
950 \\
1200\end{array}$ & $\begin{array}{r}19.5 \\
30.0 \\
39.9 \\
51.0 \\
74.5 \\
124.0\end{array}$ \\
\hline
\end{tabular}




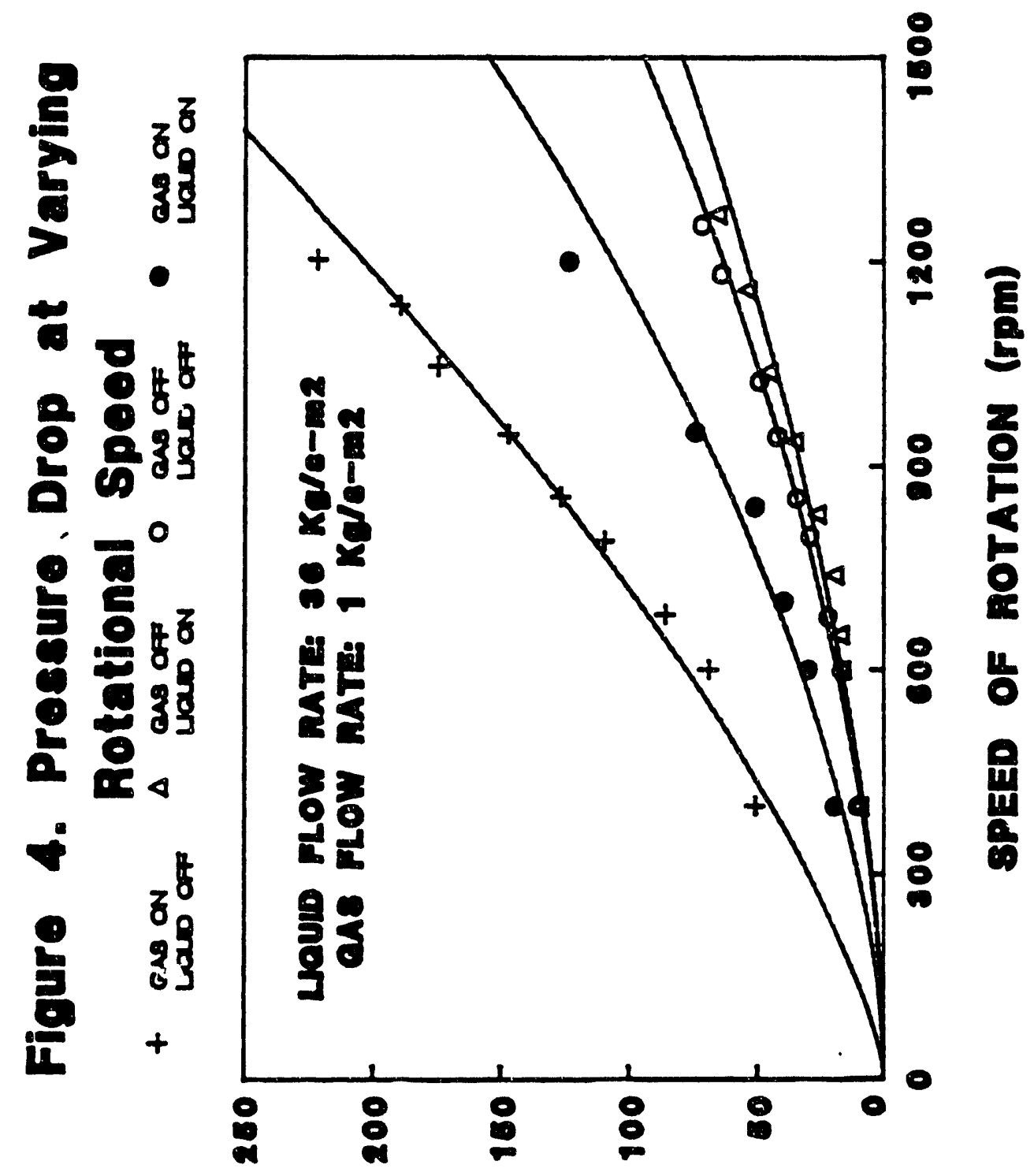

(wo/d) doua Elneseld 
gas-off are almost the same as those of the liquid-off gas-off case, indicating that liquid has no effect on pressure drop for gas-off situations. However, a decrease in the pressure drop is observed in the case of liquid-on gas-on as compared with liquid-off gas-on case. This is not a typical trend observed in conventional packed towers. In conventional towers the irrigated bed shows a higher pressure drop than the dry bed.

The solid lines in Figure 4 are indicate that the dependency of the pressure drop on rotational speed is $\Delta P \propto \omega^{2}$ at constant liquid and gas flowrates, as suggested in the proposed model.

\subsubsection{Gas Flowrate Effects:}

Pressure drop vs. gas flowrates curves were obtained at constant liquid flowrate and speed of rotation. Gas flowrates ranged from $0.1 \mathrm{~kg} / \mathrm{sec}-\mathrm{m}^{2}$ to 1.95 $\mathrm{kg} / \mathrm{sec}-\mathrm{m}^{2}$. Corresponding semi-log representation of the results (as suggested by Teutsch [23]) has iwo advantages: i) lines for constant pressure and liquid flowrate are practically straight, ii) flooding points can be clearly identified. Figure 5 shows a typical result for $12 \mathrm{~kg} / \mathrm{sec}-\mathrm{m}^{2}$ and $36 \mathrm{~kg} / \mathrm{sec}-\mathrm{m}^{2}$ liquid flowrates at 600 Ipm speed of rotation with $1476 \mathrm{~m}^{2} / \mathrm{m}^{3}$ packing specific surface area. However, the flooding points, the breakpoint in the plots where the pressure drop increases rapidly, was not reached. Liquid entrainment in the region of the distributor was encountered at the points indicated. The fact that the distributing tubes of the distributor are not placed as close as possible to the inner surface of the packing may cause the excessive entrainment of the liquid before the flooding (the liquid is 


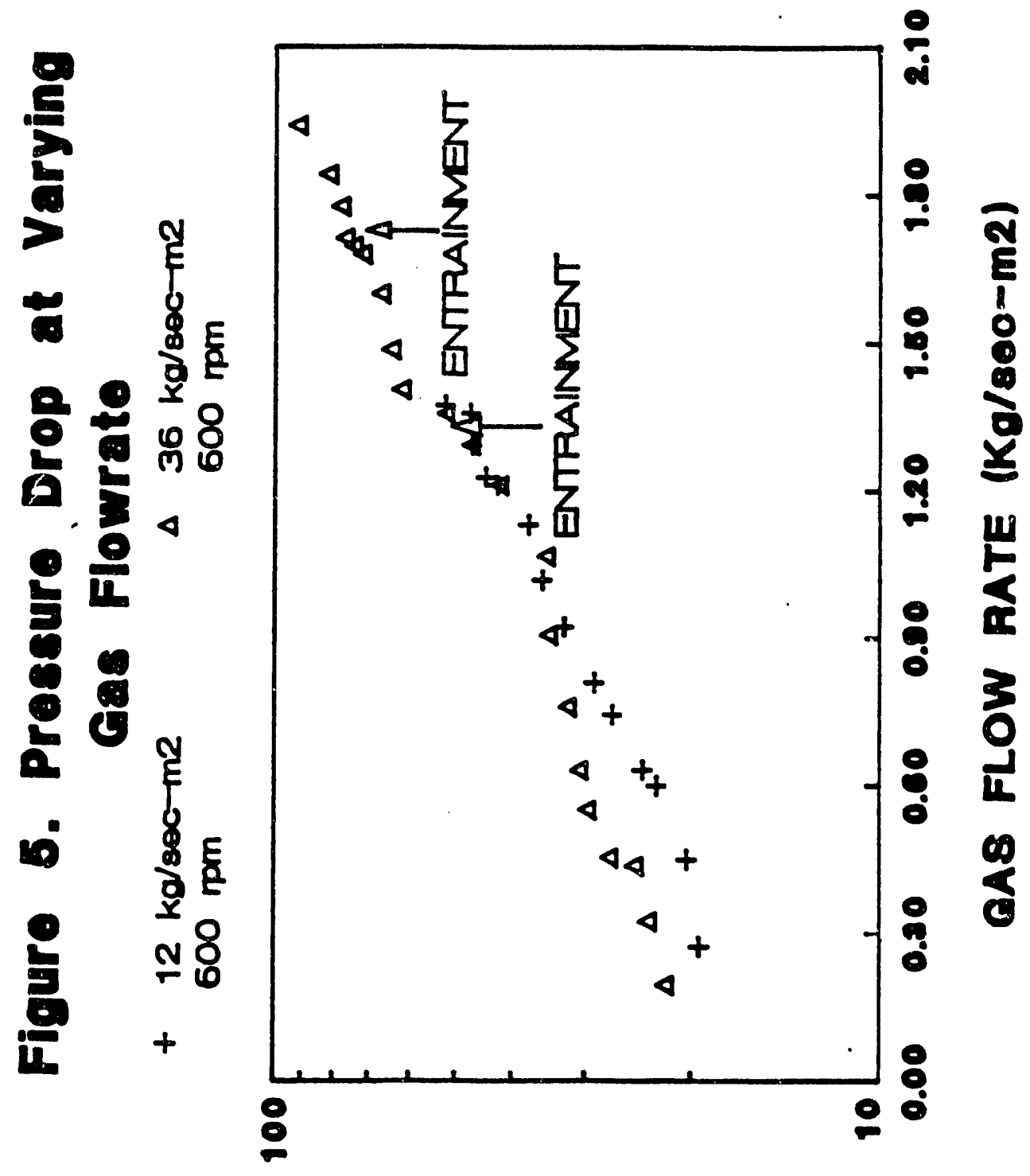

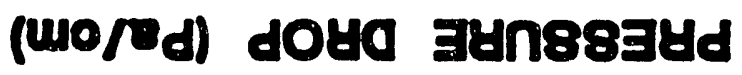


not accelerated since it does not reach the bed). Note that the change in pressure drop is not significant as the liquid flowrate is tripled. To confirm that the same pressure drop characteristics are observed at different acceleration fields, a liquid flowrate was selected $\left(36 \mathrm{~kg} / \mathrm{sec}-\mathrm{m}^{2}\right)$, and the total pressure drop was measured at varying gas flowrates for the rotational speeds of $600,840,1200 \mathrm{rpm}$ to give a gravitational acceleration of 50,100 , and $200 \mathrm{~g}$, respectively (based on inner radius of the rotor). Figure 6 shows the results. Obviously, a higher pressure drop is observed, at constant liquid and gas flowrates, for higher speed of rotation (the centrifugal force contribution to the total pressure drop). It is very clear that the curve corresponding to $1200 \mathrm{rpm}$ is not even near flooding (no entrainment was observedat full capacity liquid and gas flowrates).

\subsubsection{Liquid Flowrate Effects:}

Pressure drop versus liquid flowrates curves were obtained at constant rotational speed and gas flowrate. The results are shown in Figure 7. The pressure drop is not influenced as much by liquid flowrates as it is by gas flowrates.

\subsubsection{Effect of Packing Specific Surface Area:}

To investigate the effects of packing surface area, tests were made with different surface area packings. The packings have a specific surface area of 656 , 1476 , and $2952 \mathrm{~m}^{2} / \mathrm{m}^{3}$, all with 0.92 void fraction. The pressure drops were measured for dry and wet beds at rotational speeds of $6 j 0$ and $900 \mathrm{rpm}$. The results are shown in Figure 8. The higher pressure drops are associated with the higher surface area packing for the dry and wet beds. Note also that the dry bed shows higher pressure drop than the wet bed. 


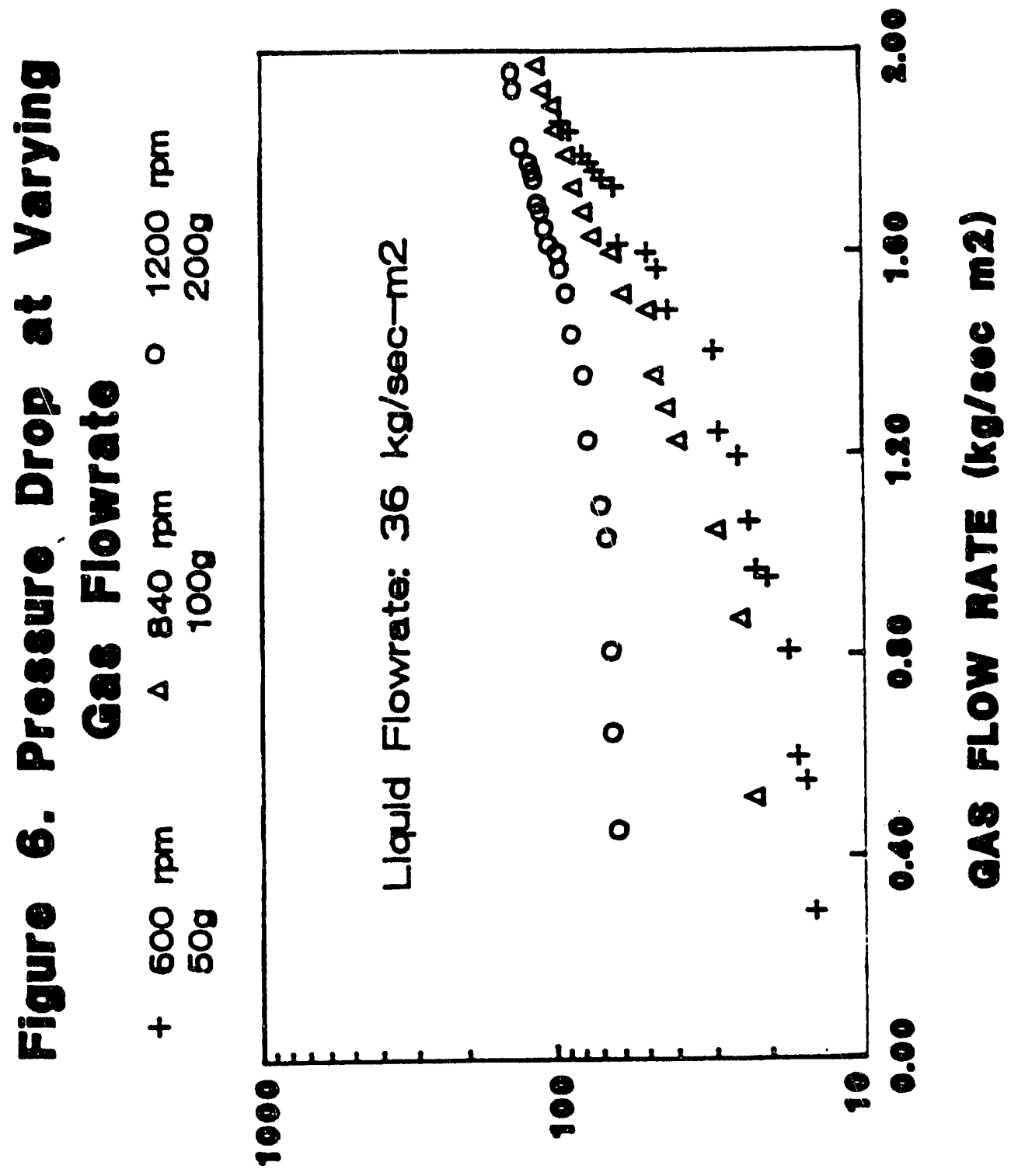

(urped) doud Eunsezyd 


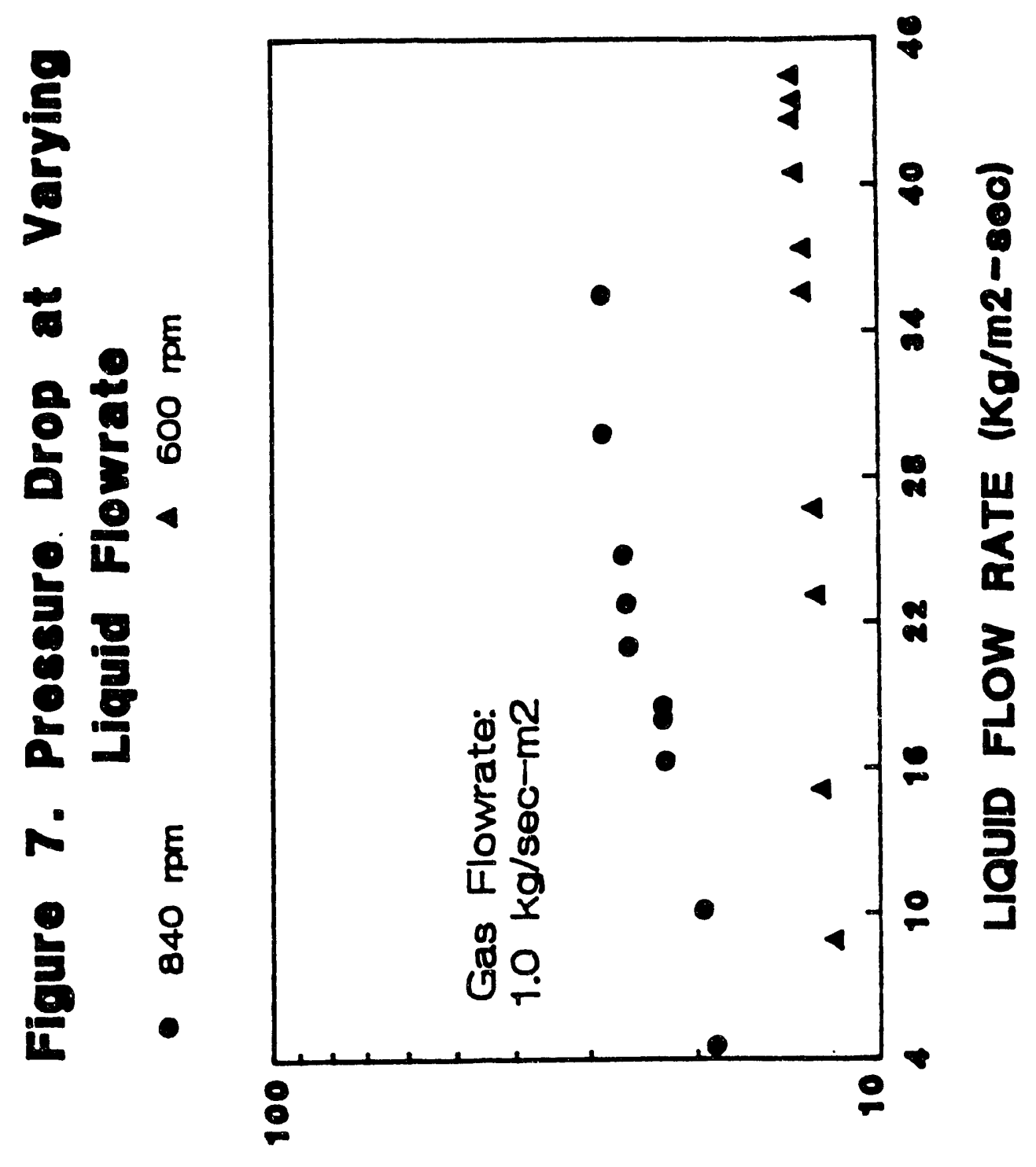

(wo/ed) doyg تtns83td 


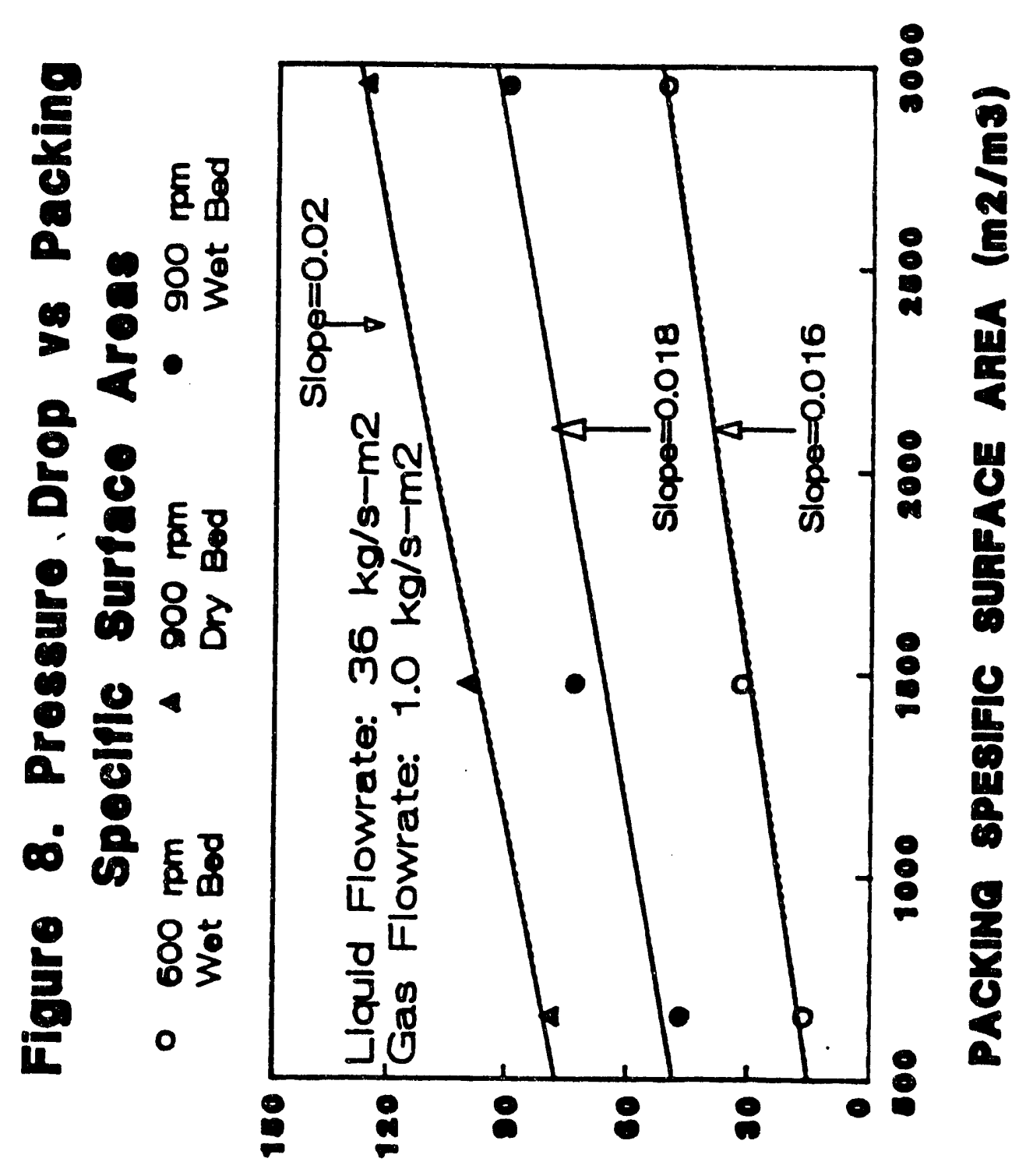

(wo/rd) doya Eunsegyd 


\subsection{Comparison with Model:}

\subsubsection{Zero Flow:}

With no gas and liquid flowing, the pressure drops were measured at varying rotational speeds. The experimental results for dry bed (gas flowrate of 1.0 $\mathrm{kg} / \mathrm{sec}-\mathrm{m}^{2}$, and packing surface area of $1476 \mathrm{~m}^{2} / \mathrm{m}^{3}$ ) are compared with the values estimated by the proposed model. Figure 9 shows this comparison.

\subsubsection{Dry Bed:}

The experimentally obtained values of the pressure drop, for dry packing, are compared with the values estimated by the proposed model in Figure 10. All of the predictions are within $\pm 20 \%$ error shown by the two broken lines in Figure

10. The experimental data was taken as the gas flowrates were varied from 0.163 to $1.31 \mathrm{~kg} / \mathrm{sec}-\mathrm{m}^{2}$, while the speed of rotation was ranging from 600 to $1200 \mathrm{rpm}$. Packing surface areas of 1476 and $2952 \mathrm{~m}^{2} / \mathrm{m}^{3}$ were used. Table 3 presents these results.

\subsubsection{Irrigated Bed:}

The experimentally obtained values of the total pressure drop, for irrigated packing, are compared with the values estimated by the model proposed for the wet packing in Figure 11. The operating conditions were similar to those of the dry packing experiments; however, the liquid flowrates were varied from 9 to 36 $\mathrm{kg} / \mathrm{sec}-\mathrm{m}^{2}$. The agreement between the calculated values and the experimental data is satisfactory (within $\pm 20 \%$ error). Although the agreement between the experimental data and the proposed model for the wet packing is promising, but 


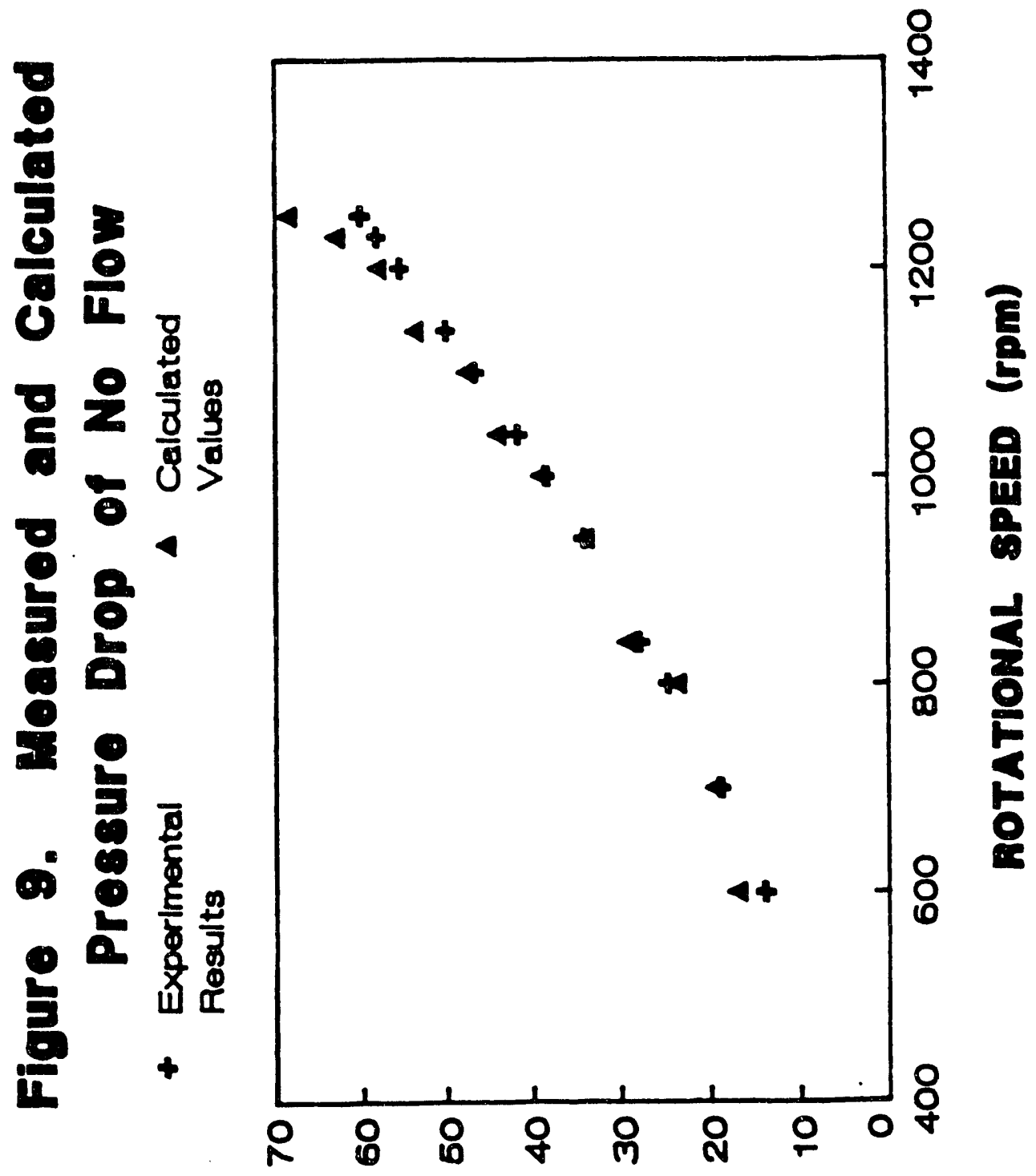

(wo/sed) doya zuns8zud 
TABLE 3. COIPARISON OF IEASURED AND CALCULATED PRESSURE DROPS AT VARYING EXPERDTENTAL CONDITIONS FOR THE DRY BED

\begin{tabular}{|c|c|c|c|c|}
\hline $\begin{array}{c}{ }^{\mathrm{a}} \mathrm{p} \\
\left(\mathrm{m}^{2} / \mathrm{m}^{3}\right)\end{array}$ & $\begin{array}{c}\text { Speed of Rotation } \\
(\mathrm{rpm})\end{array}$ & $\begin{array}{c}\text { Gas Flow } \\
\left(\mathrm{kg} / \mathrm{s}-\mathrm{m}^{2}\right)\end{array}$ & $\begin{array}{c}\Delta \mathrm{P}_{\text {measured }} \\
(\mathrm{Pa} / \mathrm{cm})\end{array}$ & $\begin{array}{c}\Delta \mathrm{P}_{\text {calculated }} \\
(\mathrm{Pa} / \mathrm{cm})\end{array}$ \\
\hline 2952 & 600 & 1.0 & 68.4 & 73.2 \\
2952 & 695 & 1.0 & 81.9 & 79.0 \\
2952 & 766 & 1.0 & 91.2 & 81.9 \\
2952 & 840 & 1.0 & 111.8 & 86.7 \\
2952 & 917 & 1.0 & 127.2 & 95.4 \\
2952 & 600 & 0.33 & 27.0 & 24.1 \\
2952 & 600 & 0.48 & 32.8 & 32.8 \\
2952 & 600 & 0.52 & 34.7 & 33.7 \\
2952 & 600 & 0.58 & 45.3 & 40.5 \\
2952 & 600 & 0.69 & 48.2 & 47.2 \\
2952 & 600 & 0.86 & 49.2 & 55.9 \\
2952 & 600 & 0.97 & 55.9 & 60.7 \\
2952 & 600 & 1.03 & 61.7 & 73.3 \\
\hline 1476 & 600 & 1.03 & 55.9 & 55.9 \\
1476 & 681 & 1.03 & 65.5 & 59.8 \\
1476 & 790 & 1.03 & 82.9 & 66.5 \\
1476 & 855 & 1.03 & 96.4 & 68.4 \\
1476 & 947 & 1.03 & 111.8 & 73.3 \\
1476 & 1040 & 1.03 & 132.1 & 80.1 \\
1476 & 1140 & 1.03 & 151.3 & 86.0 \\
1476 & 1200 & 1.03 & 167.7 & 95.0 \\
\hline 656 & 600 & 0.69 & 21.2 & 19.3 \\
656 & 600 & 0.86 & 23.1 & 21.2 \\
656 & 600 & 1.03 & 29.9 & 28.9 \\
\hline
\end{tabular}




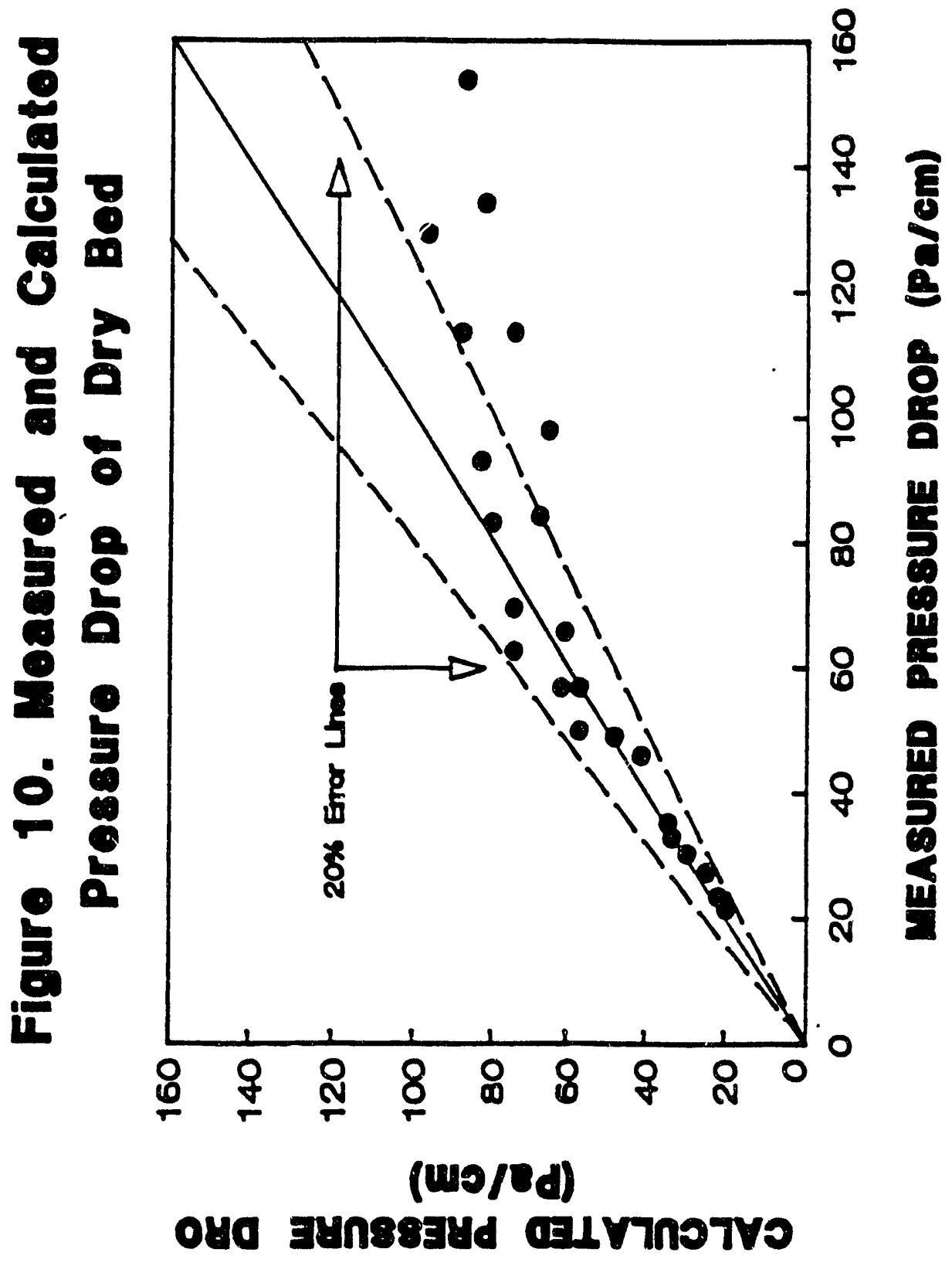




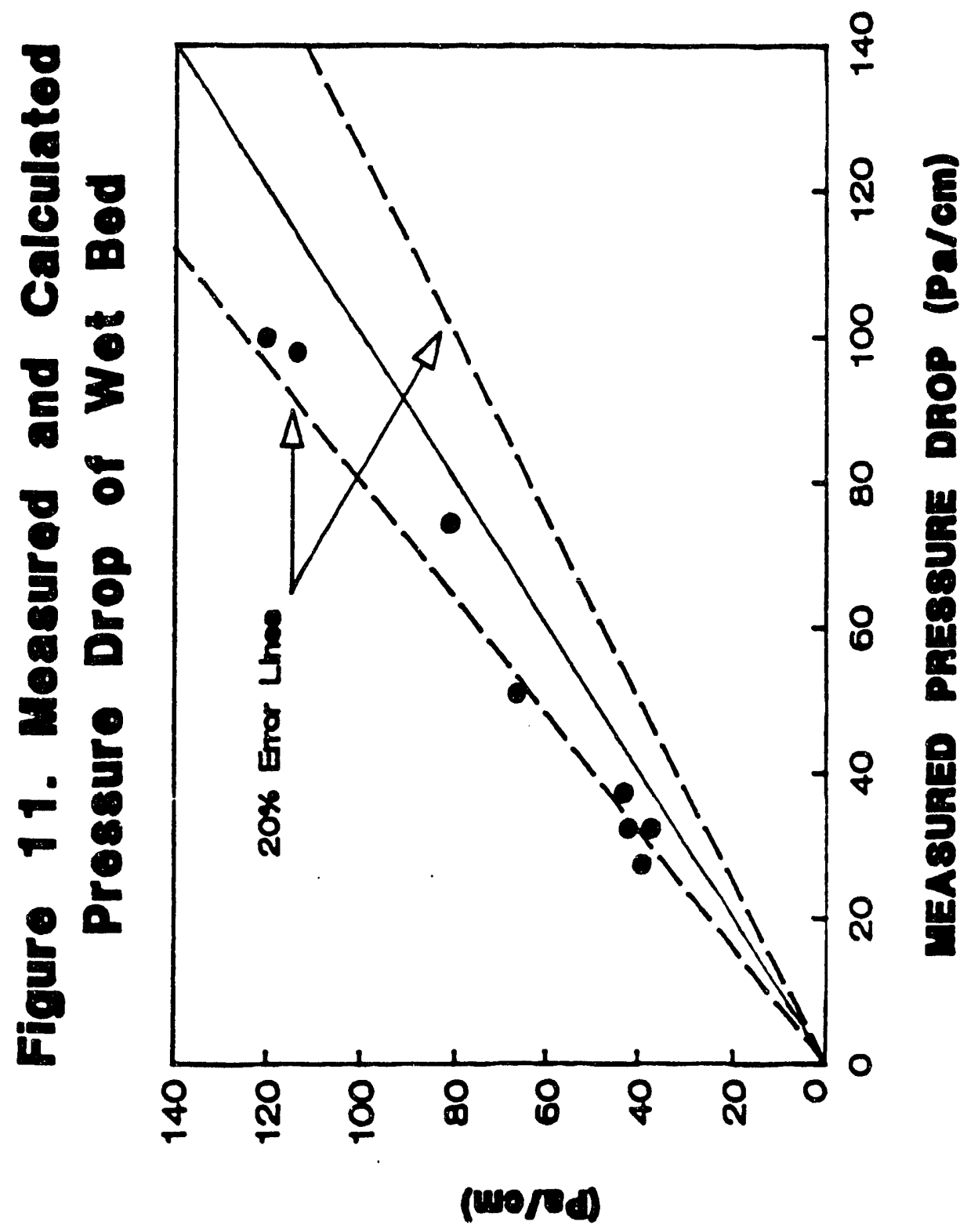

oyd EYnsogyd ordV7notyo 
the model still does not explain the lower pressure drop for the wet bed (compared to the dry bed). One possible speculation is that the liquid film exposed to the centrifugal field gets thin and behaves like a lubricant film, lowering the drag against the gas phase. Another plausible explanation is a shorter path length for the gas in the wet bed compared to the dry bed. The gas may possess smaller rotational speed in the presence of the liquid, as it enters and leaves the rotor, and as a result a lower pressure drop is observed in the wet bed.

The experimental results of pressure drop indicate that the characteristics of a rotating bed are different from those of a conventional packed bed. Therefore, the conventional analysis and known hydrodynamic relations of packed towers may not be easily applied to rotating beds.

\subsubsection{Comparison with Conventional Packed Beds:}

To evaluate further the pressure drop characteristics of rotating beds, the generalized isobaric plot of pressure drop correlation, the modified Sherwood's flooding correlation, was investigated by comparing the measured values of the pressure drop with the values obtained from the correlation (the plot) at different gas and liquid flowrates, and rotational speeds ( $g$, the gravitational field was replaced by $\omega^{2} r$, the rotational acceleration in the correlation). The rough comparison suggests that the measured values are orders of magnitude higher than the corresponding values from the curves. Figure 12 shows how these magnitudes vary at different rotational speeds. It is important to keep in mind that the values of the pressure drop in the rotating bed resulted from a variety of sources (in 


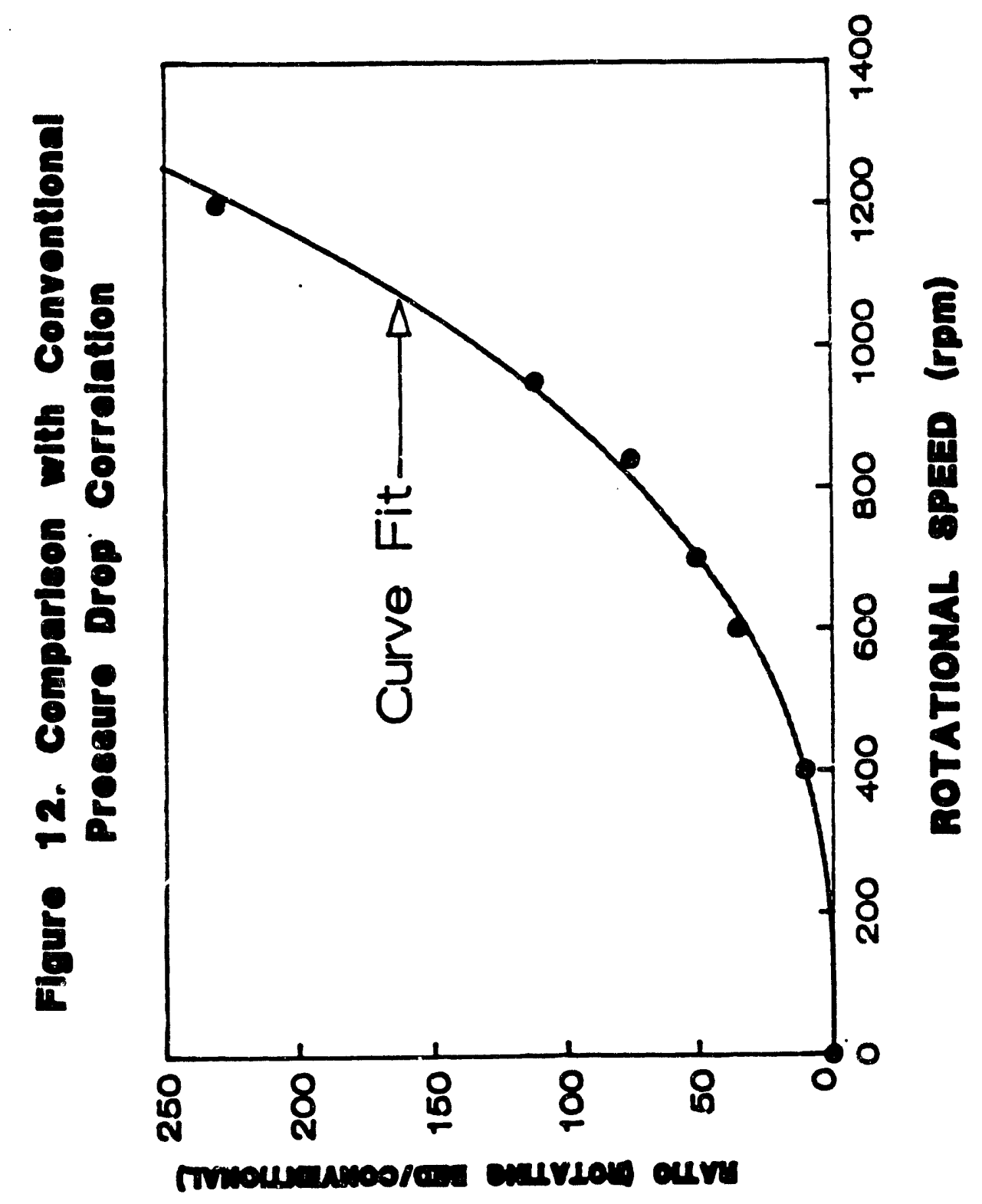


conventional columns the pressure drop is only a combination of drag forces). The air-water system was used for these experiments, and liquid flowrates ranged from 8.93 to $36 \mathrm{~kg} / \mathrm{sec}-\mathrm{m}^{2}$, and the vapor flowrate varied from 0.1 to $1.22 \mathrm{~kg} / \mathrm{sec}-\mathrm{m}^{2}$. The rotational speed varied from 600 to $1200 \mathrm{rpm}$ to achieve a range of acceleration fields of 50 to $200 \mathrm{~g}$. The packing specific surface area was $1476 \mathrm{~m}^{2} / \mathrm{m}^{3}$ with 0.92 porosity. 


\section{MASS TRANSFER CHARACTERISTICS OF ROTATING BEDS}

\subsection{Theory:}

The total height of a packed column is given by:

$$
\mathrm{H}=(\mathrm{NTU}) \times(\mathrm{HTU})
$$

where

$$
\begin{aligned}
& \mathrm{H}=\text { tower height } \\
& \text { NTU = number of transfer units } \\
& \text { HTU = height of a transfer unit }
\end{aligned}
$$

The derivation of the above equation rests on simple material balance: the rate of change of a component within a phase must be equal to the rate of transfer to the phase:

$$
d(\bar{L} \alpha)=k_{1}\left(\sigma-\sigma_{e}\right) d A
$$

Here, $\bar{L}$ is the liquid molar flowrate, $a$ is the liquid concentration, $\sigma_{\varepsilon}$ is the liquid concentration at equilibrium with the gas phase, $\mathrm{k}_{\mathrm{I}}$ is the liquid side mass transfer coefficient, and $\mathrm{dA}$ is the interfacial transfer area, which may be expressed as:

$$
d A=a_{e} S d H
$$

where $a_{e}$ is the interfacial area per unit volume of packing, $S$ is the empty tower cross-sectional area, and $\mathrm{dH}$ is the differential tower hieght. Equation (11) may 
be used to solve for the tower height required by integrating over the total change in concentration between the tower terminals:

$$
\int_{0}^{H} d H=\int_{\alpha_{0}}^{a_{q}} \frac{d(L \sigma)}{k_{1} a_{e} S\left(\alpha-\alpha_{e}\right)}
$$

Simplifications may be made by assuming that the phase flowrate, $\bar{L}$, is constant. This is a reasonable assumption for dilute systems. Since the volumetric mass transfer coefficient is a function of the this flowrate, the coefficient must be constant, providing physical properties do not vary appreciably. Thus,

$$
\int_{0}^{H} d H=\frac{\bar{L}}{k_{1} a_{e}} \int_{\delta_{0}}^{\sigma_{1}} \frac{d a}{\left(a-\alpha_{e}\right)}
$$

The integral term is equal to the total change of composition divided by the available driving force. This is defined as number of transfer units, NTU. The quantity outside the integral is called the height of a transfer unit, HTU. The tower height, $\mathrm{H}$, is the product of these two quantities as expressed in equation (10).

The above mathematical tools may be used to express the measured experimental results (for $\mathrm{Air}-\mathrm{CO}_{2}-\mathrm{Water}$ system) in terms of beight of a transfer unit for rotating packed beds, as follows: 


$$
N T U=\int_{\alpha_{0}}^{a} \frac{\mathrm{d} \alpha}{a-\alpha_{e}}
$$

where,

$a_{q}=\mathrm{CO}_{2}$ concentration in the rotor inlet

$\sigma_{0}=\mathrm{CO}_{2}$ concentration in the rotor outlet

$\varepsilon_{e}=\mathrm{CO}_{2}$ concentration in the liquid that would be at equilibrium

with $\mathrm{CO}_{2}$ concentration at the bulk gas phase

A material balance on carbon dioxide gives:

$$
\left[\left(x-\varepsilon_{0}\right)=G(y-y)\right.
$$

Here $\bar{L}$ and $\bar{G}$ are the molar water and air flowrates respectively, and $y$ is the concentration of $\mathrm{CO}_{2}$ in the rotor outlet. Assuming Henry'law to be valid for the dilute system:

$$
y=\dot{\mathrm{H}} \boldsymbol{x}
$$

where $\hat{H}$ is the Henry'law constant. Using equations (16) and (17) to solve for $\leftarrow$ :

$$
\alpha=\frac{1}{\dot{H}}\left\{\frac{\overline{\mathrm{L}}}{\bar{G}}\left(\alpha-\alpha_{0}\right)+\not \delta\right\}
$$


Substituting for from equation (18) in to equation (15), then equation (15) may be integrated to give:

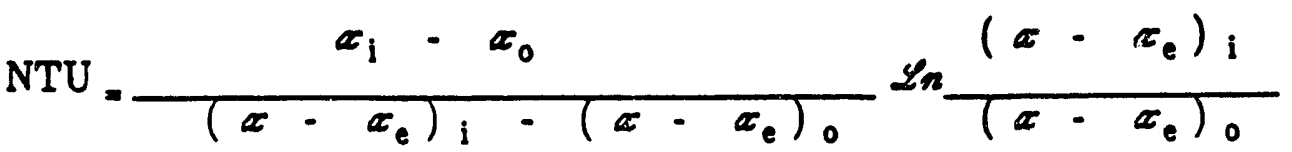

and finally to use the following expression to evaluate HTU:

$$
H T U=\left(r_{0}-r_{i}\right) / N T U
$$

Here, $r_{0}{ }_{x}$ and $r_{i}$ are the outside and inside radii of the rotating bed.

The number of transfer units is a function of the concentration of various components in the entering and exiting streams, and the phase equilibria properties of the components. The height of a transfer unit depends on liquid and gas flowrates, the nature of the packing, and the liquid and gas phase volumetric mass transfer coefficients. The number of transfer units for a given system is not changed by carrying out the separation in high gravitational fields, but the height of a transfer unit is drastically reduced because of its dependency on volumetric mass transfer coefficients, $k_{1} a_{e}, k_{1}$ is the liquid side mass transfer coefficients and $a_{e}$ is the effective gas-liquid interfacial area. The volumetric mass transfer coefficients increase because of the use of high surface area packing and the increase in the liquid side mass transfer coefficient. Dudukovic, et al showed [14] that the other contributing factor to enhance the volumetric mass transfer 
coefficients in a rotating packed bed is an increase in the effective gas-liquid contacting efficiency, $a_{e} / a_{p}$, where $a_{p}$ is the specific surface area of the packing.

Among theories proposed to describe the liquid mass transfer behavior in a packed column under conventional operation, the penetration theory appears most consistent with the picture existing in a packed tower. The penetration theory suggests that as the liquid flows over each piece of packing in laminar flow, it is completely mixed in passing from one packing piece to the next. Absorption is considered to take place during a series of brief contacts between the liquid and gas in which the dissolved gas diffuses only a short distance into the liquid film. The governing equation is:

$$
V_{i} \frac{\partial C}{\partial I} \cdot \frac{\mathscr{D}}{\partial x^{2} C}
$$

and the boundary conditions are:

$$
\begin{aligned}
& \mathrm{C}=0 \quad \mathrm{r}=0 \\
& C=0 \bigcirc x=\infty \\
& \mathrm{C}=\mathrm{C}_{0} @ \mathrm{x}=0
\end{aligned}
$$

Here, $\mathrm{C}$ is the concentration of the diffusing component, $\mathrm{V}_{\mathrm{i}}$ is the interfacial velocity of the liquid film flowing on the wall, $\mathscr{D}$ is the diffusion coefficient, $\mathrm{C}_{0}$ is the interfacial concentaration, $\mathrm{r}$ is the radial direction, and $\mathrm{x}$ is the axial direction (perpendicular to r direction).. 
Solving equation (21) under boundary conditions of (22) will result in the following expression for $k_{1}$, the liquid side mass transfer coefficient:

$$
k_{1}=\sqrt{49 / \pi t}
$$

where $t$ is the time during which the surface of the liquid is exposed to the gas and can be estimated by the following equation:

$$
\mathrm{t}=\pi \mathrm{Z} / 2 \mathrm{~V}_{\mathrm{i}}
$$

where $\mathrm{Z}$ is the packing length characteristic. The interfacial velocity, $\mathrm{V}_{\mathrm{i}}$, may be estimated by stream-line flow theory as

$$
V_{i}=\left(9 g \Gamma^{2} / 8 \mu_{1} \rho\right)^{1 / \beta}
$$

where $\Gamma$ is the liquid flowrate per unit width, $\mu_{1}$ is the liquid viscosity, and $\rho$ is the

- liquid density. Equation (25) is obta ned under assumptions of negligible interfacial shear and the complete mixing of the liquid at the junctions of packing pieces.

Substituting equation (25) into equation (24), and equation (24) into equation (23), results in the following expression for liquid side mass transfer coefficient: 


$$
\frac{k_{1} Z}{\mathscr{D}}=0.918\left(a_{p} / a_{e}\right)^{1 / 3} \mathrm{Sc}^{1 / 2} \operatorname{Re}^{1 / 3} \mathrm{Gr}^{1 / 6}
$$

where,

$$
\begin{aligned}
\mathrm{Sc} & =\text { Schmidt number }=\mu_{1} / \rho \mathscr{D} \\
\mathrm{Re} & =\text { Reynolds number }=\mathrm{L} / \mathrm{a}_{\mathrm{p}} \mu_{1} \\
\mathrm{Gr} & =\text { Grashof number }=\mathrm{Z}^{3} \rho^{2} \mathrm{~g} / \mu_{1}^{2}
\end{aligned}
$$

Here, $\mathrm{L}$ is the superficial liquid mass flowrate.

The liquid side mass transfer coefficient, $k_{1}$, may be evaluated using equation (26), after the effective gas-liquid contacting efficiency, $a_{e} / a_{p}$, is estimated. In estimating the effective gas-liquid contacting efficiency, $a_{e} / a_{p}$, it is important to keep in mind that the gas-liquid interfacial surface area is influenced by the body force. The fact that the interfacial area is sensitive to liquid flowrates, as most of the correlations in the literature predict, suggests it is likely that the interfacial area would be dependent on the body force. Dudukovic et al, showed that the effective surface area increases with the body force with 0.14 to 0.28 power [14]. Therefore, among the suggested correlations the one that takes into account the influence of the body force on the effective interfacial area should be used.

Among several formulae proposed to correlate interfacial areas, the most general one seems to be the equation by Onda et al [24] that predicts the wetted surface area and not only takes into account surface tension and the surface energy of the packing, but also has a gravity term built in to the correlation: 
$a_{e} / a_{p}=a_{w} / a_{p}=1-\exp \left\{-1.45\left(\sigma_{d} / \bar{\sigma}\right)^{0.75} \operatorname{Re}^{0.1} \mathrm{Fr}^{-0.05} \mathrm{We}^{0.2}\right\}$

where,

$a_{w}=$ wetted surface area of the packing

$\bar{\sigma}=$ surface tension of liquid

$\sigma_{c}=$ critical surface tension of packing material

$\mathrm{Fr}=$ Froude number $=\mathrm{L}^{2} \mathrm{a}_{\mathrm{p}} / \rho^{2} \mathrm{~g}$

$\mathrm{We}=$ Weber number $=\mathrm{L}^{2} / \rho \bar{\sigma} \mathrm{a}_{\mathrm{p}}$

Equation (17) combined with equation (18) may be used to evaluate liquid side volumetric mass transfer coefficient in rotating packed bed by replacing gravitational acceleration, $g$, with rotational acceleration, $a_{r}=\omega^{2} r_{i}$, and liquid flowrate per unit width with liquid flowrate per perimeter. The major assumption is that the interfacial surface area of the gas-liquid, $a_{e}$, is the same as the packing wetted area, $a_{w}$. The reported experimental values of $a_{e}$ by Dudukovic et al [14] may be estimated, within $7 \%$ to $10 \%$, using this correlation.

Then, the following equation will be used to estimate the value of height of a transfer unit, HTU:

$$
H T U=L / \rho k_{1} a_{e}
$$

Another conventional analysis that may be applied to rotating beds is the theoretical work made by Davidson [25]. Davidson was the first to consider a 
theoretical approach to evauate liquid side mass transfer coefficient by developing three statistical models to represent random packings. He used the penetration theory to make quantitative calculations for packed towers. In the vertical surface model, the packing was assumed to be made up of a large number of vertical surfaces of height $Z$ which were completely and equally wetted. The model provides an expression for HTU:

$$
\mathrm{HTU} / \mathrm{Z}=0.345 \mathrm{Sc}^{1 / 3} \mathrm{Re}_{1}^{2 / 3} \mathrm{Gr}^{-1 / 6}
$$

Here, $R e_{1}=4 \mathrm{~L} / \mu \mathrm{a}_{\mathrm{e}}$. He then extended this model to cases of random angle, and random angle and length, and he developed similar equations for all the cases, with different constants. This model may be used for rotating beds by replacing the appropriate terms as was suggested for the earlier model. Note that to evaluate $R e_{1}, a_{e}$, the effective gas-liquid interfacial area should be estimated. Equation (27) may be used to estimate $a_{e}$.

Both of these models give a very simple picture of what could be happening in a rotating bed; complications such as actual flow pattern of the fluids, Coriolis force, fluid distribbution, and packing shape are all neglected. On the other hand, this approach may provide some tools to reveal some of the significant differences between the rotating beds and conventional packed towers.

\subsection{EXPERIMENTAL RESULTS AND DISCUSSION: $\quad-\mathrm{CO}_{2}$ in $W_{\text {ate }}$}

Experiments are performed to investigate the effects of operating parameters, particularly the acceleration field, the packing specific surface area, 
and the liquid and gas loading, on HTU. About 75 experimental runs were made at varying operating conditions. The results of the mean values of HTU with the standard error of the mean are summarized in Table 4. The results of all the experiments are presented in the Appendix.

\subsubsection{Gravitational Effects:}

Experimental runs were made as the liquid and gas flowrates were beld constant at 36 and $1.0 \mathrm{~kg} / \mathrm{sec}-\mathrm{m}^{2}$, while the rotor speed of rotation was set at 600 , 850,1130 , and $1385 \mathrm{rpm}$ to achieve a centrifugal acceleration of $50,100,182$, and 272g, respectively (based on the inner radius of the rotor). All three beds were used. Tre influence of the acceleration field on HTU is shown in Figure 13 which indicates the dependency of HTU on $\mathrm{g}$ varies from -0.3 to -0.4 , within the range estimated by Dudukovic et al $[14,15]$ and Vivian et al $[6]$. Comparing the slopes of the lines for different packing surface areas suggests that the dependency of HTU on the acceleration field is more pronounced at higher surface area packings.

\section{2..2. Effect of Pacling Specific Surface Area:}

The contribution of packing specific surface area on enhanced volumetric mass transfer coefficients is shown in Figure 14. The liquid and gas flowrates for all the experimental runs were set at $36 \mathrm{~kg} / \mathrm{sec}-\mathrm{m}^{2}$ and $1.0 \mathrm{~kg} / \mathrm{sec}-\mathrm{m}^{2}$, respectively, while the rotational speeds were set at $600,850,1130$, and $1385 \mathrm{rpm}$ to achieve a centrifugal acceleration of $50,100,182,272 \mathrm{~g}$, respectively (based on the inner radius of the rotor). The measured volumetric mass transfer coefficients of $2952 \mathrm{~m}^{2} / \mathrm{m}^{3}$, in all different accelerations, are greater than the corresponding 
TABLE 4. EXPERDTENTAL DATA AT VARYTNG OPERATING CONDITIONS

\begin{tabular}{|c|c|c|c|c|c|}
\hline $\begin{array}{c}\text { Liquid Flow } \\
\left(\mathrm{kg} / \mathrm{sec}-\mathrm{m}^{2}\right)\end{array}$ & $\begin{array}{c}\text { Gas Flow } \\
\left(\mathrm{kg} / \mathrm{sec}-\mathrm{m}^{2}\right)\end{array}$ & $\# \mathrm{~g}$ & $\begin{array}{c}\mathrm{a}_{\mathrm{p}} \\
\left(\mathbf{m}^{2} / \mathrm{m}^{3}\right)\end{array}$ & $\begin{array}{c}\text { ETU } \\
(\mathrm{cm})\end{array}$ & $\begin{array}{c}\text { Standard } \\
\text { Error of Mean }\end{array}$ \\
\hline 18 & 1.0 & 50 & 1476 & 3.0 & 0.052 \\
36 & 1.0 & 50 & 1476 & 3.75 & 0.020 \\
36 & 1.0 & 50 & 2952 & 2.42 & 0.076 \\
36 & 1.0 & 50 & 656 & 2.74 & 0.019 \\
36 & 0.66 & 50 & 1476 & 2.63 & 0.088 \\
\hline 18 & 1.0 & 100 & 1476 & 2.24 & 0.051 \\
36 & 1.0 & 100 & 1476 & 2.94 & 0.177 \\
36 & 1.0 & 100 & 2952 & 1.97 & 0.038 \\
36 & 1.0 & 100 & 656 & 2.20 & 0.000 \\
36 & 0.66 & 100 & 1476 & 2.10 & 0.052 \\
\hline 18 & 1.0 & 182 & 1476 & 1.97 & 0.012 \\
36 & 1.0 & 182 & 1476 & 2.45 & 0.132 \\
36 & 1.0 & 182 & 2952 & 1.67 & 0.014 \\
36 & 1.0 & 182 & 656 & 2.10 & 0.050 \\
36 & 0.66 & 182 & 1476 & 1.92 & 0.020 \\
\hline 18 & 1.0 & 272 & 1476 & 1.79 & 0.003 \\
36 & 1.0 & 272 & 1476 & 2.0 & 0.000 \\
36 & 1.0 & 272 & 2952 & 1.30 & 0.095 \\
36 & 1.0 & 272 & 656 & 1.92 & 0.085 \\
36 & 0.66 & 272 & 1476 & 1.62 & 0.030 \\
\hline
\end{tabular}




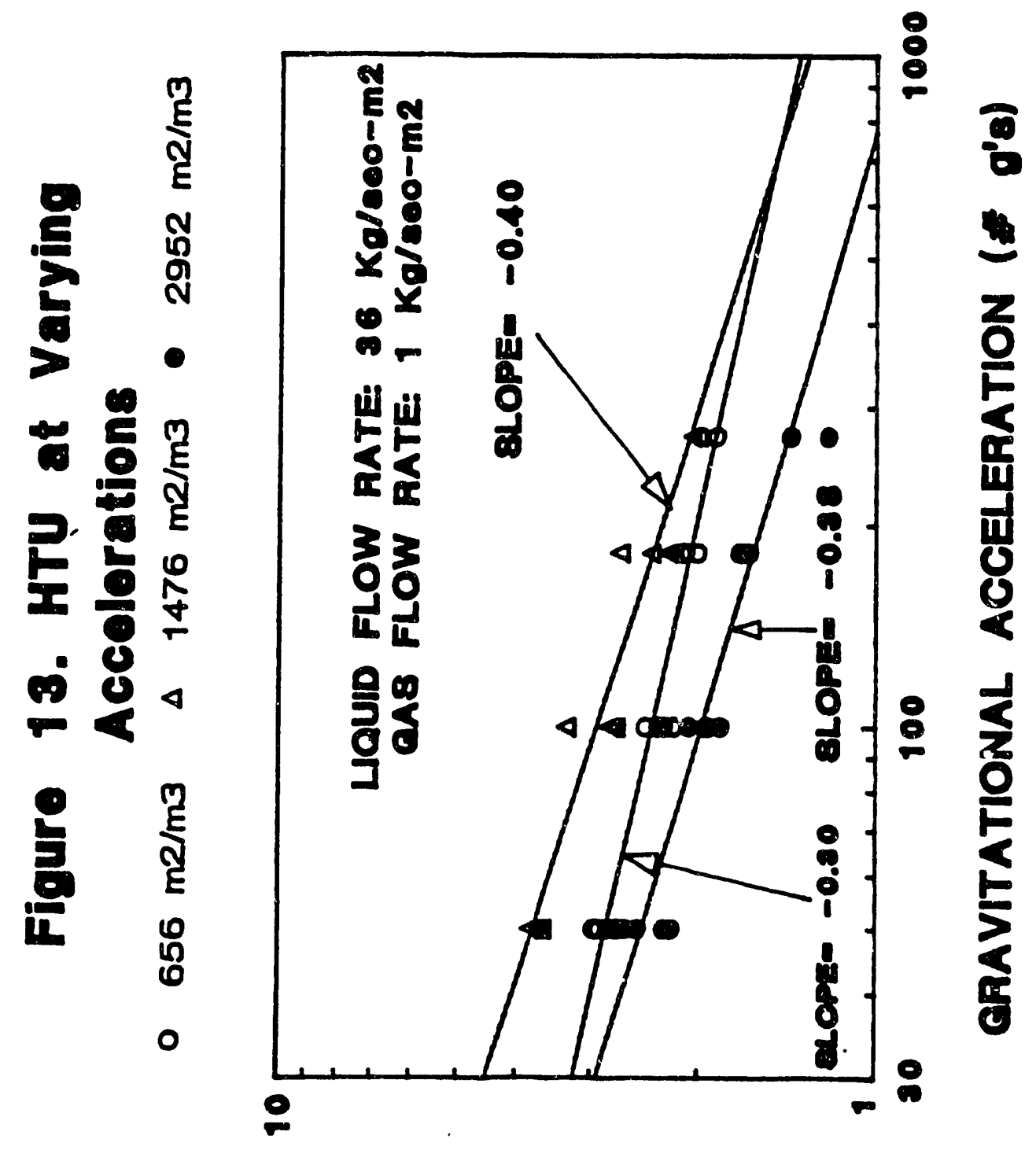

(no) nut 


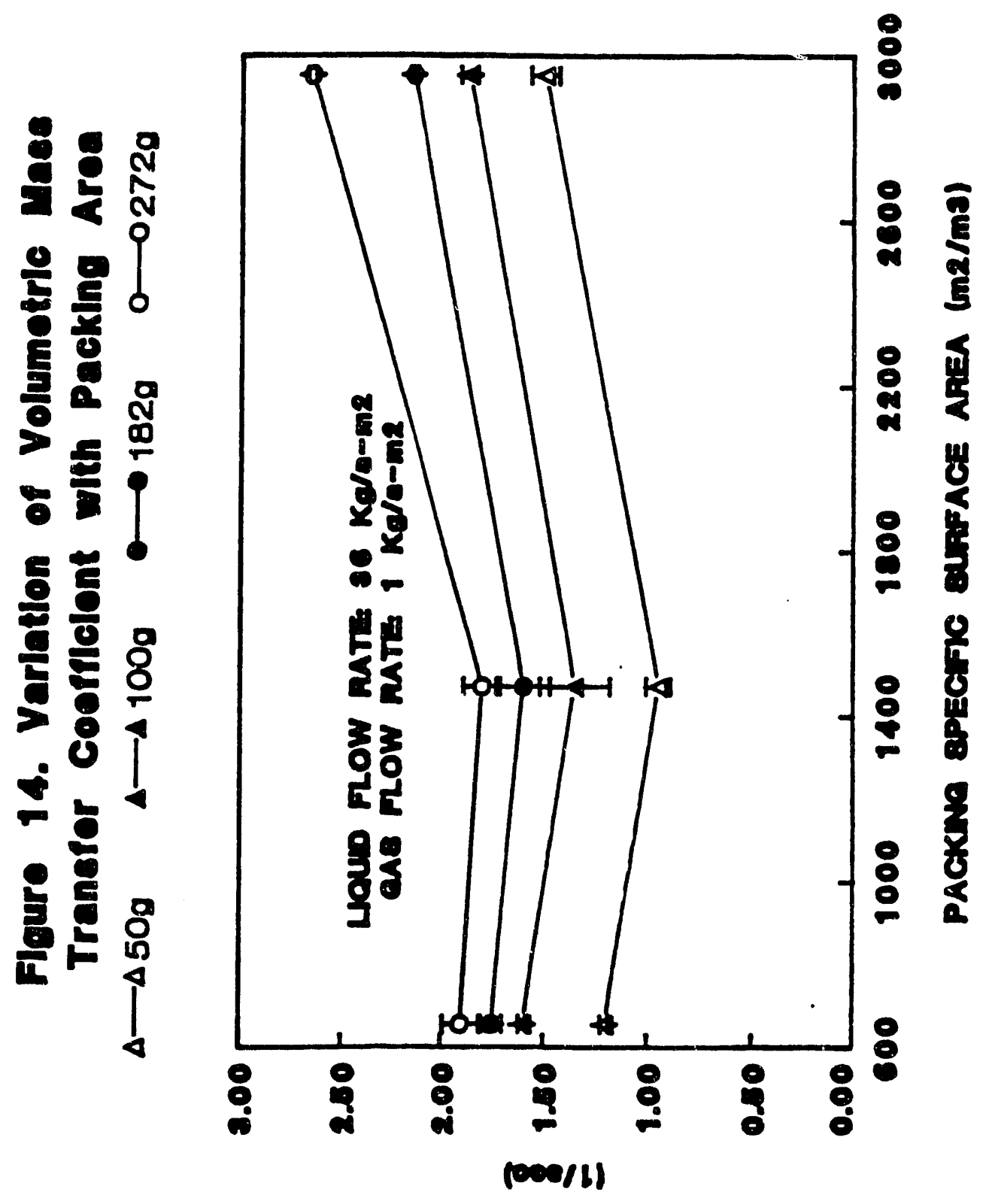

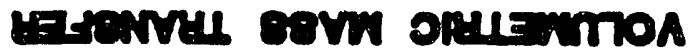


packing will result in higher volumetric mass transfer coefficients. However, as can be seen in Figure 14, the results for $656 \mathrm{~m}^{2} / \mathrm{m}^{3}$ packing do not show the same trend. The volumetric mass transfer coefficients in the case of $656 \mathrm{~m}^{2} / \mathrm{m}^{3}$ packing are slightly greater those of $1476 \mathrm{~m}^{2} / \mathrm{m}^{3}$ (or comparable within the experimental errors). This discrepancy may be attributed partly to end effects: the fact that the mass transfer cannot be confined to the packing alone. Some mass transfer may take place as the fine droplets leave the rotor and travel to the housing. Another factor which may contribute to the higher experimental volumetric mass transfer coefficients for $656 \mathrm{~m}^{2} / \mathrm{m}^{3}$ (compared to those of $1476 \mathrm{~m}^{2} / \mathrm{m}^{3}$ ) is that the liquid spread in the tangential direction due to the Coriolis force is more pronourced for $656 \mathrm{~m}^{2} / \mathrm{m}^{3}$ packing with larger pore size (allowing larger deviation from the the radial direction). This means that the wetted surface area, $a_{w}$, may be higher for $656 \mathrm{~m}^{2} / \mathrm{m}^{3}$ packing than that of $1476 \mathrm{~m}^{2} / \mathrm{m}^{3}$ packing, resulting in higher volumetric mass transfer coefficients. More work needs to be done to reinforce the above statements.

\subsubsection{Effect of Liquid Flowrate:}

To investigate the liquid flowrate effects, the HTU dependency on the acceleration fields was determined at liquid flowrates of $36 \mathrm{~kg} / \mathrm{sec}-\mathrm{m}^{2}$ and 18 $\mathrm{kg} / \mathrm{sec}-\mathrm{m}^{2}$. The gas flowrate was held at $1.0 \mathrm{~kg} / \mathrm{sec}-\mathrm{m}^{2}$, and the packing with specific surface area of $1476 \mathrm{~m}^{2} / \mathrm{m}^{3}$ was used. The results are presented in Figure 15. The slope of the best line drawn through these data, using least square fit technique, is -0.33 for higher liquid flowrate in comparison with the slope of -0.30 at the lower liquid flowrates. Note that higher values of HTU were obtained at higher liquid flowrate, something to be expected. 


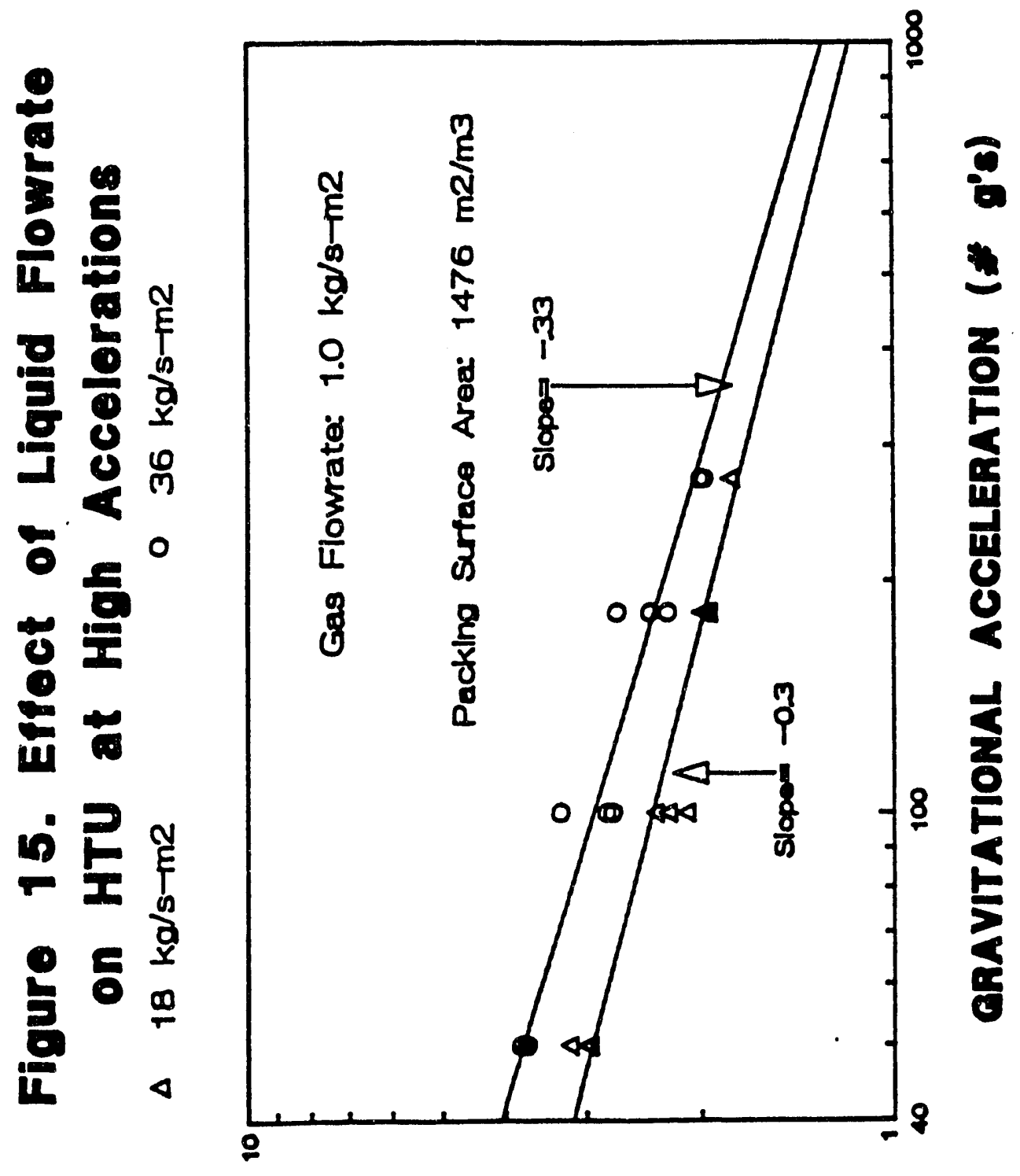

(ve) nLA 


\subsubsection{Effect of Gas Flowrate:}

Tests were also performed at gas flowrate of $0.66 \mathrm{~kg} / \mathrm{sec}-\mathrm{m}^{2}$ at varying centrifugal accelerations, while the liquid flowrate kept constant at $36 \mathrm{~kg} / \mathrm{sec}-\mathrm{m}^{2}$. The results of these tests are presented in Figure 16. Although lower values of volumetric mass transfer coefficients were measured for these experiments in comparison with the higher flowrate $\left(1.0 \mathrm{~kg} / \mathrm{sec}-\mathrm{m}^{2}\right)$, the power dependency of HTU on gravitational field turned out to be the same as before (slope of the line is -0.30). It is important to point out that the fact that HTU increases as the gas flowrate decreases is consistent with the trend observed in conventional columns.

\subsubsection{Effect of Viscosity:}

Increasing the liquid viscosity decreases mass transfer rate due to reduced diffusivity and mixing in the liquid phase. To investigate the effect of viscosity on mass transfer in rotating beds, a solvent-bevane-air system was studied, where the hexane was air stripped from the solvent at $70^{\circ} \mathrm{C}$ and 1 atm. The solvent viscosity was $3 \mathrm{cp}$ at $70^{\circ} \mathrm{C}$.

At rotational speeds of 700 to $1100 \mathrm{rpm}$, HTU ranges from $1.5-2.5 \mathrm{~cm}$ were achieved. The results are presented in Table 5. The packing with specific surface area of $1476 \mathrm{~m}^{2} / \mathrm{m}^{3}$ was used.

\subsection{Comparison with Models:}

The experimentally obtained values of HTU from all the performed experiments at varying gravitational fields, as well as different packing surface areas, and varying gas and liquid llowrates, are compared with the HTU values estimated from both models, using equations (26) to (29). The results are presented in Table 6. This comparison is illustrated in Figure 17. Figure 17 shows 


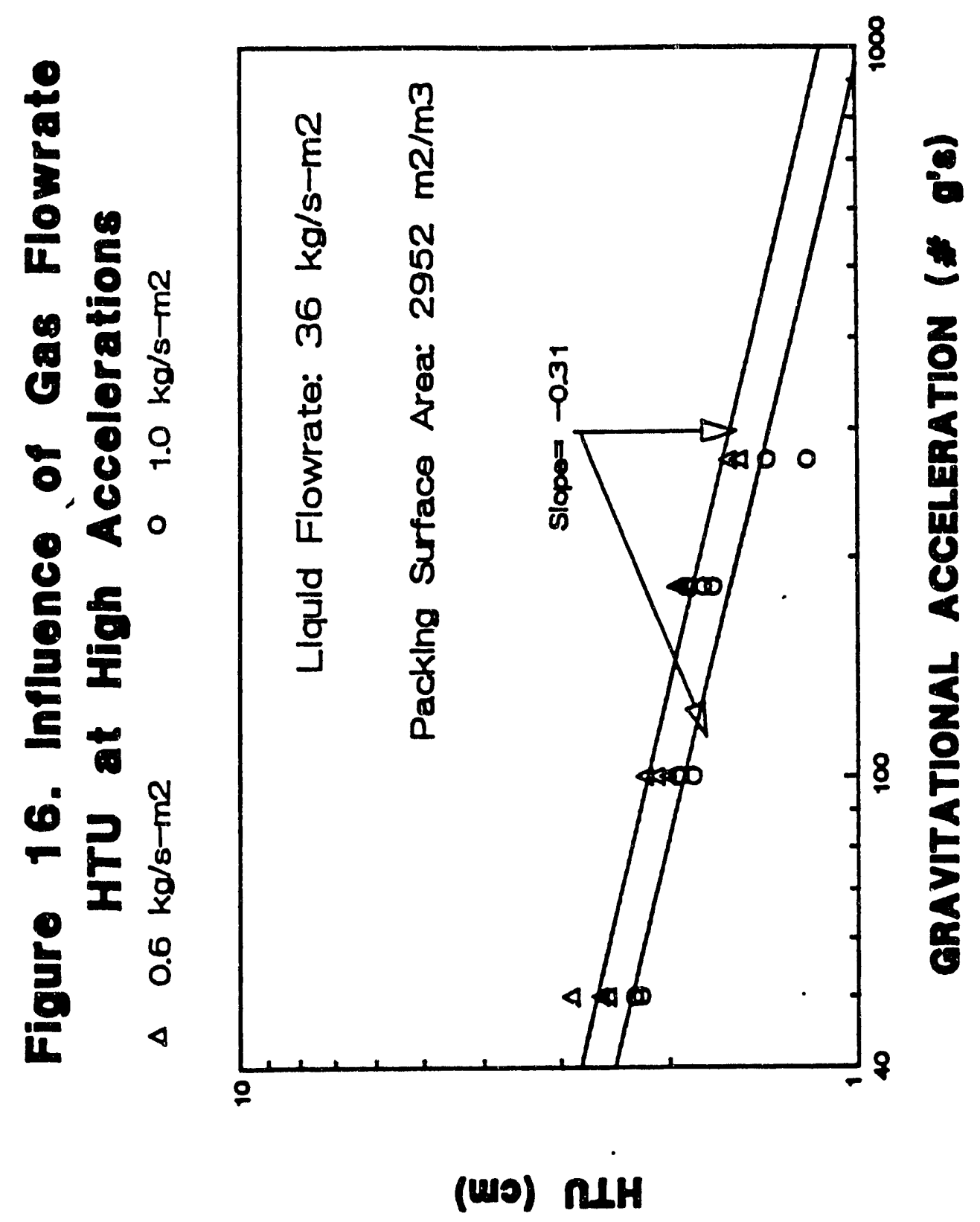


TABLE 5. EXPERTENTAL RESOLTS OP HAB VISCOSITY SOLVENT

\begin{tabular}{|c|c|c|c|c|c|}
\hline $\begin{array}{c}T_{\text {liquid }} \\
(0 \mathrm{C})\end{array}$ & $\begin{array}{l}T_{\text {gas }} \\
\left(\begin{array}{ll}0 & C\end{array}\right)\end{array}$ & $\begin{array}{c}\text { Liquid Flou } \\
\left(\mathrm{Xg} / \mathrm{s}-\mathrm{B}^{2}\right)\end{array}$ & $\begin{array}{l}\text { Gas Flow } \\
\left(\mathrm{Xg} / \mathrm{s}-\mathrm{n}^{2}\right)\end{array}$ & $\mathrm{fg}_{\text {ave. }}$ & stages \\
\hline $\begin{array}{l}79.3 \\
84.2 \\
87.0 \\
85.7 \\
84.7 \\
82.1 \\
86.2 \\
86.9 \\
90.5 \\
87.8 \\
85.2 \\
89.0 \\
93.0 \\
86.4\end{array}$ & $\begin{array}{l}92.8 \\
88.9 \\
93.9 \\
94.5 \\
88.9 \\
93.9 \\
94.4 \\
94.4 \\
94.4 \\
94.4 \\
93.9 \\
88.9 \\
88.9 \\
93.9\end{array}$ & $\begin{array}{l}28 \\
26 \\
26 \\
29 \\
26 \\
28 \\
29 \\
29 \\
29 \\
28 \\
28 \\
27 \\
27 \\
26\end{array}$ & $\begin{array}{l}1.10 \\
1.20 \\
1.12 \\
1.11 \\
1.11 \\
1.10 \\
1.12 \\
1.11 \\
1.11 \\
1.11 \\
1.12 \\
1.12 \\
1.12 \\
1.12\end{array}$ & $\begin{array}{r}75 \\
75 \\
75 \\
75 \\
165 \\
184 \\
208 \\
343 \\
447 \\
474 \\
388 \\
422 \\
539 \\
150\end{array}$ & $\begin{array}{l}4.15 \\
4.39 \\
4.13 \\
1.80 \\
6.18 \\
7.90 \\
10.14 \\
12.60 \\
14.0 \\
13.2 \\
6.50 \\
7.50 \\
12.50 \\
5.63\end{array}$ \\
\hline
\end{tabular}


TABLE 6. COYPARISON OF VEASURED AND GALCULATED VALLES OF HTU

\begin{tabular}{|c|c|c|c|}
\hline$\# g$ & $\begin{array}{c}\text { Measured HTU } \\
\text { (cm) }\end{array}$ & $\begin{array}{l}\text { Calculated ATU (ca) } \\
\text { Using Penetration Theory }\end{array}$ & $\begin{array}{l}\text { Calculated ITU (ca) } \\
\text { Davidson's Model }\end{array}$ \\
\hline \multicolumn{4}{|c|}{ LIQUID FLOH: $36 \mathrm{Kg} / \mathrm{s}-\mathrm{m}^{2}$, GAS FLOH: $1.0 \mathrm{Kg} / \mathrm{s}-\mathrm{m}^{2}, a_{\mathrm{p}}: 1476 \mathrm{~m}^{2} / \mathrm{m}^{3}$} \\
\hline \begin{tabular}{|r|}
50 \\
100 \\
182 \\
272 \\
\end{tabular} & $\begin{array}{l}3.67 \\
2.80 \\
2.30 \\
2.00\end{array}$ & $\begin{array}{l}3.29 \\
2.92 \\
2.50 \\
2.40\end{array}$ & $\begin{array}{l}3.60 \\
3.10 \\
2.80 \\
2.60\end{array}$ \\
\hline \multicolumn{4}{|c|}{ LIQUTD FLOH: $18 \mathrm{Kg} / \mathrm{s}-\mathrm{m}^{2}$, GAS FLOH: $1.0 \mathrm{Kg} / \mathrm{s}-\mathrm{m}^{2}, a_{\mathrm{p}}: 1476 \mathrm{~m}^{2} / \mathrm{m}^{3}$} \\
\hline 50 & 2.98 & 2.50 & 2.70 \\
\hline 100 & 2.25 & 2.19 & 2.39 \\
\hline 182 & 1.98 & 1.92 & 2.10 \\
\hline 272 & 1.79 & 1.83 & 2.00 \\
\hline \multicolumn{4}{|c|}{ LIQUTD FLOY: $36 \mathrm{Kg} / \mathrm{s}-\mathrm{m}^{2}$, GAS FLOH: $1.0 \mathrm{Kg} / \mathrm{s}-\mathrm{m}^{2}, a_{\mathrm{p}}: 2952 \mathrm{~m}^{2} / \mathrm{m}^{3}$} \\
\hline 50 & 2.22 & 1.58 & 1.71 \\
\hline 100 & 1.83 & 1.41 & 1.51 \\
\hline 182 & 1.67 & 1.22 & 1.33 \\
\hline 272 & 1.22 & 1.16 & 1.26 \\
\hline \multicolumn{4}{|c|}{ LIQUID FLOH: $36 \mathrm{Kg} / \mathrm{s}-\mathrm{m}^{2}$, GAS FLOH: $1.0 \mathrm{Kg} / \mathrm{s}-\mathrm{m}^{2}, a_{\mathrm{p}}: 656 \mathrm{~m}^{2} / \mathrm{m}^{3}$} \\
\hline 50 & 2.80 & 7.01 & 5.60 \\
\hline 100 & 2.19 & 6.09 & 5.00 \\
\hline 182 & 2.01 & 5.48 & 4.40 \\
\hline 272 & 1.96 & 5.18 & 4.2 \\
\hline
\end{tabular}




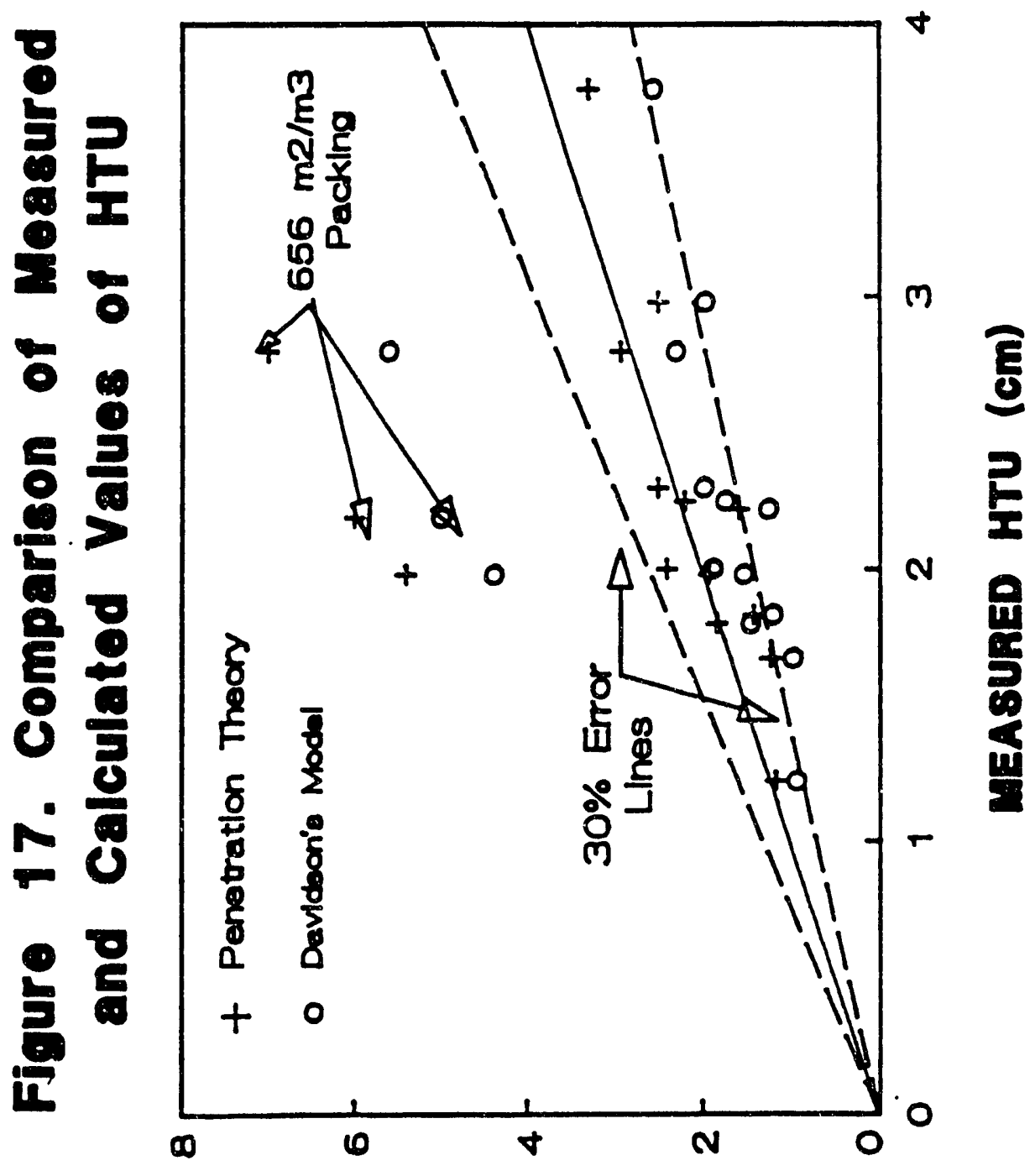

(wo) กLH aELYרก) 
the results are within \pm 30 , except for the results of $656 \mathrm{~m}^{2} / \mathrm{m}^{3}$ packing. It is interesting to observe that the estimated values agree fairly well with the experimental values. Neither model seems to show any superiority over the other within these range of experimental conditions. However, Davidson's model seems to underpredict the HTU values. The good agreement between the estimated and exper inental values is nut observed for the values corresponding to $656 \mathrm{~m}^{2} / \mathrm{m}^{3}$ packing because of fossible reasons discussed earlier. 


\section{POWER REQUIREMENT FOR ROTATING BEDS}

\subsection{THEORY:}

The power consumption arises from three sources: 1) the poiver required for the acceleration of the liquid to the tip speed of the rotor; 2) the power consumed in overcoming the frictional windage drag of the vapor; and 3) the power required for the bearing friction. In addition, there is a small power requirement associated with the flow of vapor between the rotating disk and the stationary housing, and the flow of vapor through the rotor bed.

\subsubsection{Bearing Friction:}

The power consumed by the bearing friction will be strongly dependent upon the type of the bearing used. It is reasonable to assume that the frictional loss of, bearing is directly proportional to speed of rotation. The proportionality may be evaluated experimentally.

\subsubsection{Windage Effect:}

Windage losses may be evaluated by studying the flow between two disks, one spinning and one stationary. There have been numerous studies of this type of flow in the literature [17-21]. In particular, the flow pattern of disk.in a housing with large gap between them (width of the gap compared with the radius of the disk) was investigated for the laminar and for the turbulent flow by Von F. Shultz-Grunow [20]. It was suggested that for a large gap, an additional boundary layer will be formed on the stationary housing, as well as on the rotating disk. The 
fluid in the boundary layer on the revolving disk is centrifuged outwards and this is compensated by a flow inwards in the boundary layer on the stationary housing. For the turbulent flow case, the tangential velocity was assumed to obey the 1/7-th-power law. The expressions for the torque both for the laminar and turbulent cases are as follows:

$$
\begin{array}{ll}
T=2.67 \rho_{\mathrm{g}} \omega^{2} R^{5}(R e)^{-1 / 2} & \text { Laminar } \\
T=0.0622 \rho_{\mathrm{g}} \omega^{2} R^{5}(R e)^{-1 / 5} & \text { Turbulent }
\end{array}
$$

Here, $T$ is the torque, $\rho_{g}$ is the gas density, $R$ is the disk radius, and $R e$ is Reynold's number defined as:

$$
\operatorname{Re}=\rho_{\mathrm{g}} \omega \mathrm{R}^{2} / \mu_{\mathrm{g}}
$$

where $\mu_{\mathrm{g}}$ is the gas viscosity.

The power is, of course, the product of the torque and and angular velocity:

$$
\text { Power }=\mathrm{T} \times \omega
$$

\subsubsection{Windage Effect with Inflow:}

The flow pattern for a rotating disk in a stationary housing is modified when there is inflow $[17,19]$. There is a significant influence of inflow on shear stresses 
at the wall and the disk [17]. The fluid rotates faster due to inflow, and the tangential shear stress is reduced at the rotating disk and increased at the stationary housing (similarly for the radial shear stresses). The influence of inflow on shear stresses is more pronounced for larger gap width. The windage loss may be defined as follows:

$$
\text { Power }=\int_{r_{\mathfrak{i}}}^{r_{0}} 2 \pi r^{2} \omega \tau_{t d} d r
$$

where $\tau_{\text {td }}$ is the tangential stress on the rotating disk, $r_{0}$ and $r_{i}$ are the outside and inside radii respectively, and $r$ is the radial direction. From equation (32) it can be seen that the power loss due to windage is reduced due to inflow since $\tau_{\text {td }}$ is reduced. Von. F. Shultz-Grunow [20] proposed the following expression for $\tau_{\text {to }}$ :

$$
\tau_{t d}=F_{t} \rho_{g} \frac{(r \omega-U)^{2}}{2}
$$

where $F_{t}$ is friction factor defined as:

$$
\begin{aligned}
& \mathscr{S}_{t}=0.0996(\mathrm{Re})^{-1 / 5} \times\left(\frac{1}{1-\mathrm{r} / \mathrm{r}_{0}}\right)^{2 / 5} \quad \text { Turbulent } \\
& \mathscr{S}_{t}=3.66(\mathrm{Re})^{-1 / 2} \times\left(\frac{1}{1-\mathrm{r} / \mathrm{r}_{0}}\right) \quad \text { Laminar }
\end{aligned}
$$


Here, $U$ is the tangential velocity. The knowledge of tangential velocity profile is needed in order to evaluate $\tau_{t d}$. The equation of motion in tangential direction may be written as follows:

$$
\rho_{g} V \frac{d U}{d r}-\rho_{B} \frac{U V}{I}=\left(\tau_{t d}-\tau_{t w}\right) / S
$$

Here, $\mathrm{V}$ is the radial velocity, $\mathrm{s}$ is the gap between the rotor and the stationary housing, and $\tau_{\text {tw }}$ is the tangential shear stress on the wall of stationary housing defined as follows:

$$
\tau_{t w}=\tau_{t} \rho_{8} \frac{U^{2}}{2}
$$

Equations (33) to (37) will be used to evaluate the tangential velocity, then the power may be estimated using equation (32). A $4^{\text {th }}$ order Runge-Kutta method was used to solve equation (36). Equation (32) was solved numerically using the composite trapezoidal rule.

\subsubsection{Liquid Acceleration:}

The largest contributor to power consumption is the acceleration of the liquid to the top speed of the rotor. The theoretical power required to bring the liquid to angular velocity of the rotor may be calculated as follows:

$$
\text { Power }=\text { Cbange in Kinetic Energy }=(\text { K.E. })_{\text {out }}-(\text { K.E. })_{\text {in }}
$$


Assuming that the liquid enters the rotor with no angular velocity, $(\text { K.E. })_{\text {in }}=0$, the power may be estimated as:

$$
\text { Power }=(\text { K.E. })_{\text {out }}=I \omega^{2} / 2
$$

Here, $I$ is the moment of inertia $=m r_{\text {out }}^{2}$, where $m$ is the liquid flowrate, and $r_{\text {out }}$ is the outside radius of the rotor. $\omega$ is the angular velocity of the rotor $=2 \pi \mathrm{N}$, where $\mathrm{N}$ is the rotational speed.

\subsection{Experimental Results and Discussion:}

Figure 18 shows the total power consumption as a function of liquid flowrate at three different accelerations.

In order to sort out the factors contributing to the power consumption, experiments were performed to isolate the contributors.

\subsubsection{Bearing Friction:}

To isolate the bearing contribution of power consumption, the rotor was dismantled from the shaft, and the power was measured as the shaft was allowed to rotate in the open housing. Figure 19 presents the results. It can be seen that it is reasonable to assume that the bearing frictional loss is directly proportional to rotational speed, Power $=0.2(\mathrm{rpm})$, for our equipment.

\subsubsection{Windage Effects:}

To investigatc the windage effects, the power measurements were made at varying acceleration fields (varying speed of rotation) with the liquid and gas flows off. The bearing power consumption was subtracted. The experimentally obtained values of the power were compared with the values estimated by 
Figure 18. Total Power as a Function of Liquid Flowrate + $600 \mathrm{rm}$ \ $840 \mathrm{~mm}$ - $960 \mathrm{~mm}$

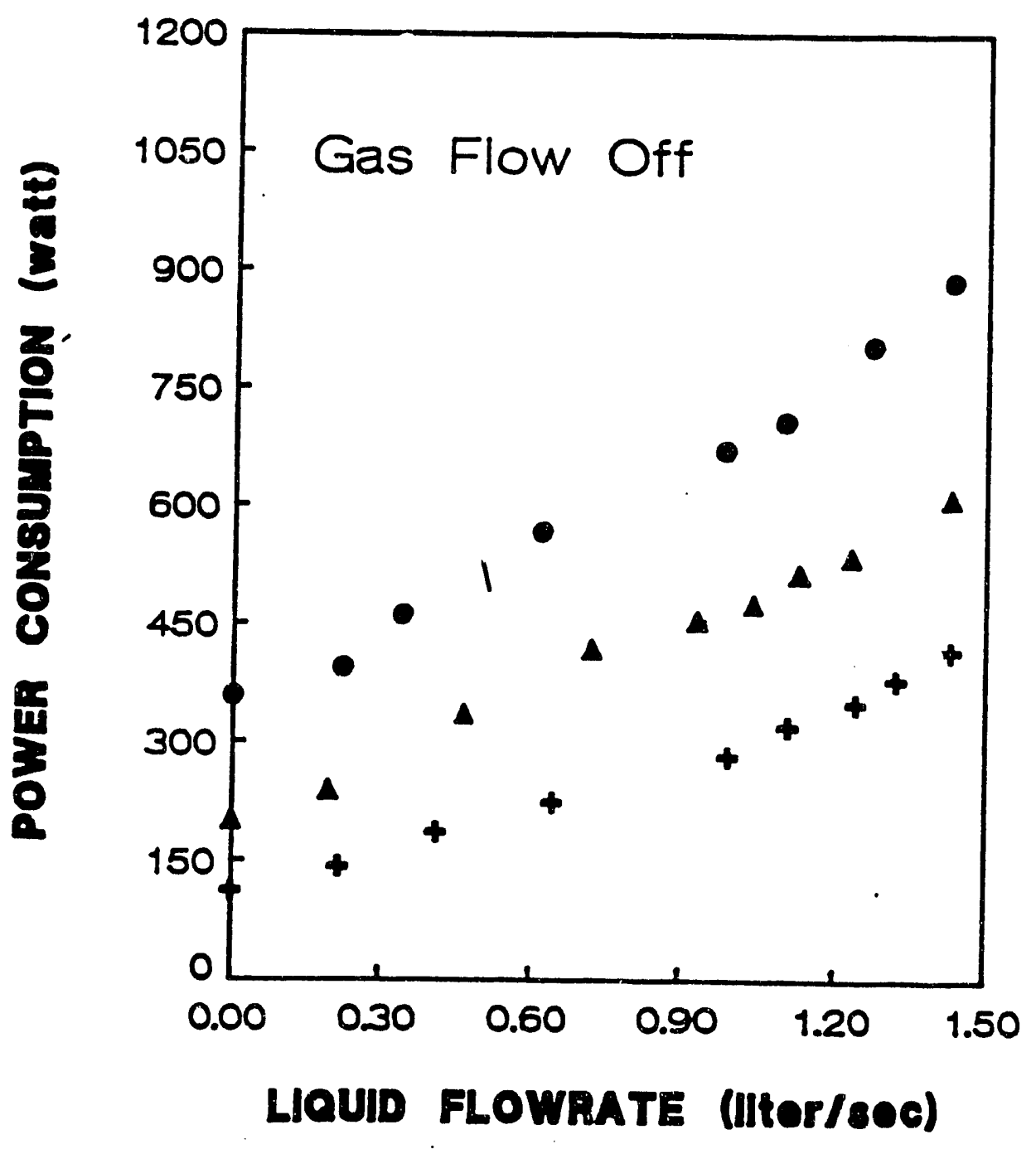


Flgure 10. Windage and Boaring Power Consumption ve Rotational speed
$\Delta$ Bearing
Effect
- Windage
Effect

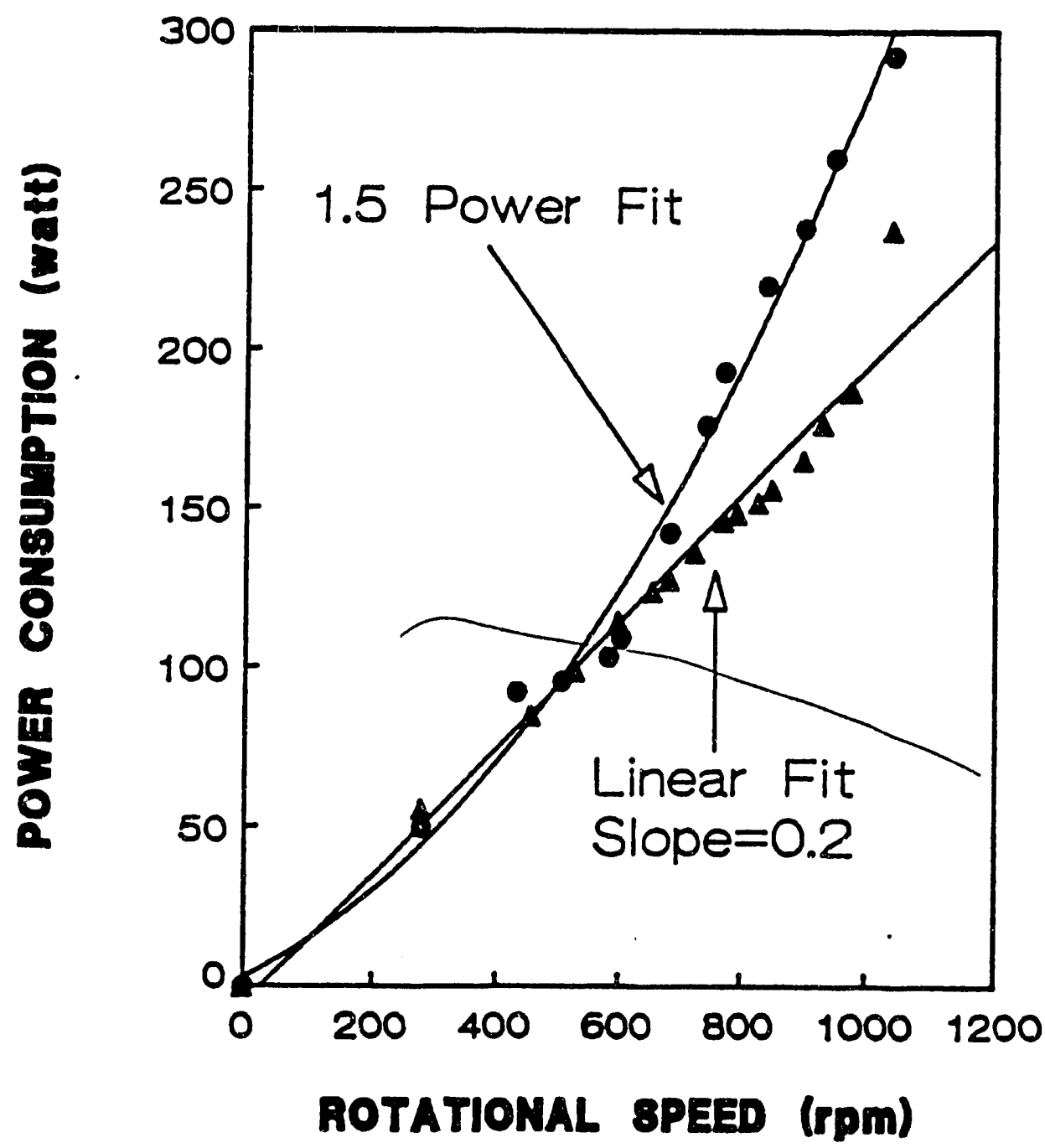


equations (20) and (21) proposed by Von. F. Shultz-Grunow [20]. The model predicts extremely low values for the windage effects compared with the experimental values (order of magnitude less at low speeds). The model predicts that the windage loss varies with the cube of rotational speed. However, Figure 19 shows a $3 / 2$ power dependency of power consumption on speed, Power $=8.79 \times 10^{-3}(\mathrm{rpm})^{1.5}$. One of the factors for the low prediction may come from the mismatch of the apparatus setup. Shultz-Grunow's disk was free within housing, while in our setup the rotor is engaged to a lip seal, which in turn, tends to increase the power consumption due to frictional loss.

\subsubsection{Windage Effect with Inflow:}

The power consumption was measured with gas flow-on and liquid flow-off at varying accelerations. The bearing power consumption was subtracted. These experimentally obtained values of power were compared with the estimated values of the windage effect with inflow as discussed in Section 6.1.3. The results are presented in Figure 20. The rotational speed was varied from 400 to $1200 \mathrm{rpm}$, and the gas flow rate ranged from 0.34 to $1.34 \mathrm{~kg} / \mathrm{sec}-\mathrm{m}^{2}$.

\subsubsection{Liquid Acceleration:}

Experiments were carried out to measure the power at varying rotational speeds at three different liquid flowrates, 1.40, 1.0, and $0.65 \mathrm{liter} / \mathrm{sec}$, and zero gas flowrate. The bearing and windage power consumption were subtracted. The results are shown in Figure 21. 


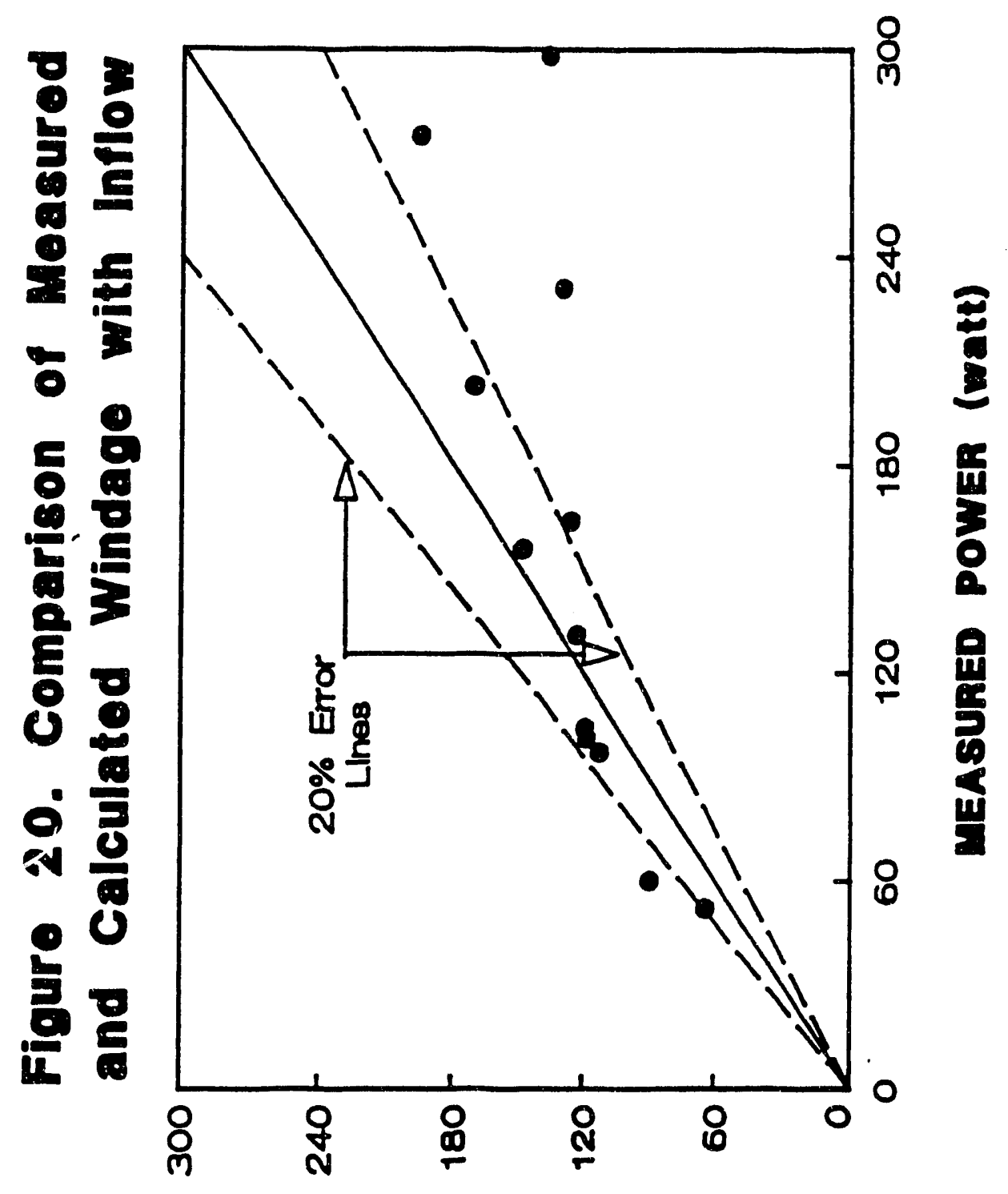

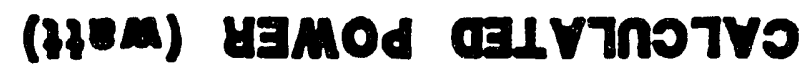


Figure 21. Power Consumed for Liquid Acceleration

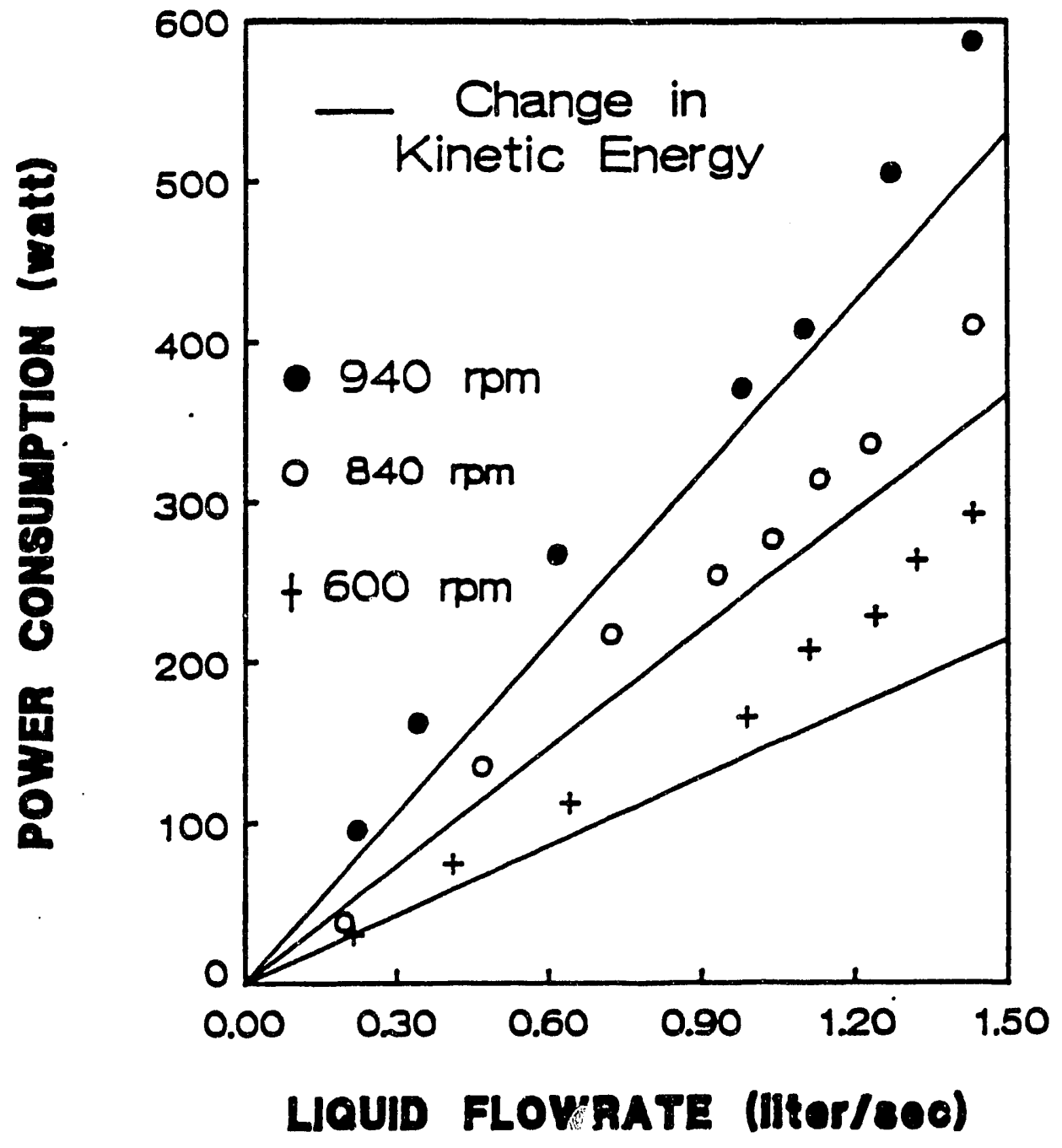




\subsubsection{Total Power Consumption:}

Based on the results of all the components contributing to the power consumption, the total power may be written as:

$$
\text { Total Power }=a w^{2}+b w^{1 \cdot 5}+c w
$$

where $a, b$, and $c$ are constants. The total power was calculated from the empirical equation derived for the bearing and windage effects, and the theoretical power required for the liquid acceleration ( change in kinetic energy $=I \omega^{2} / 2$ ). The calculated values are compared with the experimentally obtained values in Figure

22. The difference between the calculated and experimental values is, partly, the result of loss of energy through conversion of mechanical energy to beat. Note also that a reduction in total power consumption is obtained when the gas flows through the rotor. This norm is mainly a result of the inflow effect as discussed earlier. 
Figure 22. Total Power (contribution of all componenta)

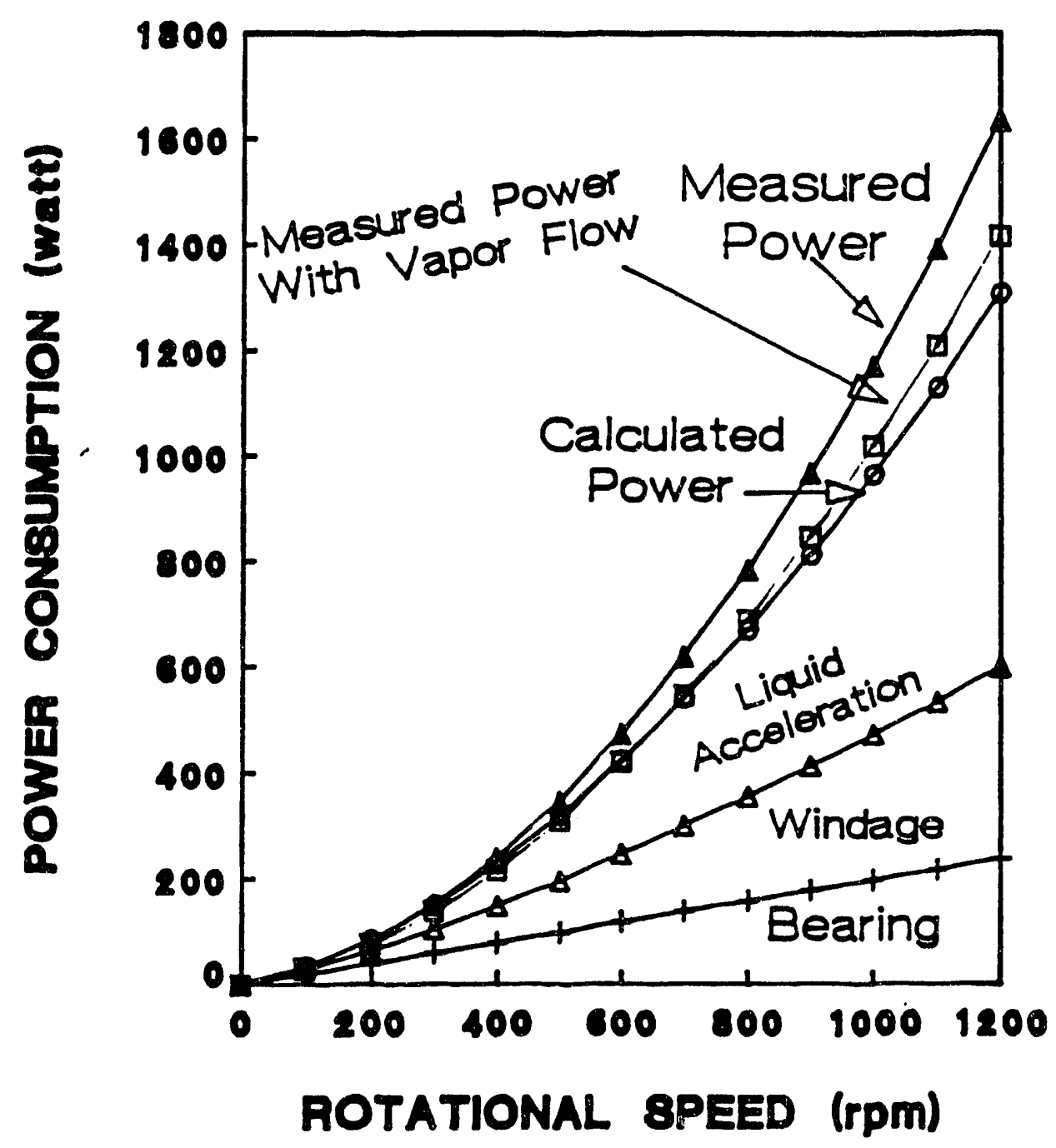




\section{Residence Time Distribution}

\subsection{Theory:}

Residence time distribution (RTD) analysis has been a valuable tool in obtaining information on the fluid motion and mixing in a continuous flow system $[25,26]$. The experimental technique is a stimulus-response technique. The stimulus is a tracer input into the fluid entering the vessel, whereas the response is a time record of the tracer leaving the vessel. A perfect tracer has exactly the same flow properties as the substance it represents, yet it is sufficiently different in some nonflow attribute that it can be detected by an analytical instrument. In aqueous systems the choice usually falls on electrolytes. When a slug of tracer enters the bed, velocity variations, molecular diffusion and turbulent mixing all tend to spread the measured response of the tracer at the bed outlet. The term dispersion is used to denote the combined action of ali the dispersive phenomena. Numerous models have been used in the literature to simulate systems with varying degrees of dispersion [26-32]. The proposed models can be divided into two groups: a dispersion model which has been used for empty tubes and packed beds, and a combined model in which complete mixing and plug flow regions are located in series and parallel arrangements. It has been shown in the literature $[30,31]$ that the dispersion model can successfully simulate the behavior of devices in which complex radial and axial flow and transport patterns exist.

\subsubsection{Dispersion Model:}

The dispersion model assumes a plug flow pattern with no radial variations in velocity and concentration. The dispersion occurs in the axial direction and the extent of the dispersion is sufficient to account for the combined effects of all 
dispersive phenomena (turbulent mixing, molecular diffusion, and nonuniform velocities) in the device. The spread in the impulse response takes place in the axial direction with an effective diffusivity, $D_{e}$. For $D_{e}=0$ the model reduces to ideal plug flow, indicating no axial dispersion. When $D_{e} \rightarrow \infty$, axial dispersion dominates and the model approaches to perfect mixer conditions.

The derivation of the model equations ranging from the most general to the most restricted are discussed in the literature [30-32]. The dimensional analysis of the dispersion model results in a dimensionless group: the Peclet number, defined as follows:

$$
\mathrm{Pe}=\frac{\overline{\mathrm{V}} \mathrm{L}_{0}}{\mathrm{D}_{e}}
$$

where $\bar{V}$ is superficial velocity, $L_{0}$ is a characteristic length of the device. The degree to which axial dispersion influences the performance of the device is determined by the value of the Peclet number, Pe. A high value of Pe corresponds to a low dispersed flow ( $\mathrm{Pe} \rightarrow \infty$, or $\left.\mathrm{D}_{\mathrm{e}} \rightarrow 0\right)$, while a low value of $\mathrm{Pe}$ represents a high dispersed flow $\left(P e-0\right.$, or $\left.D_{e} \rightarrow \infty\right)$.

Statistical techniques such as method of moments may be used for interpretation of the experimental data. The following mathematical expressions are used to characterize the data: 


$$
\begin{aligned}
& \bar{t}=\left(\int_{0}^{\infty} t C(t) d t\right) /\left(\int_{0}^{\infty} C(t) d t\right) \\
& \sigma^{2}=\left(\int_{0}^{\infty}(t-\bar{t})^{2} C(t) d t\right) /\left(\int_{0}^{\infty} C(t) d t\right)
\end{aligned}
$$

Here, $\bar{t}$ is the mean value or the centroid of the distribution, $\sigma$ represents the spread of the distribution, and $\mathrm{C}(\mathrm{t})$ is the measured response at the outlet of the device for the tracer impulse injected at the inlet. However, since it is not always possible to inject a tracer as a perfect impulse, an imperfect pulse method (also known as two peak method) may also be employed. The technique involves monitoring the concentration at two points in the test section (the bed inlet and outlet) instead of at a single point. The injection point may be located anywhere upstream of the monitoring stations. The convolution properties may be used to calculate the mean residence time and the variance of the device as follows:

$$
\begin{aligned}
& \bar{t}_{\text {bed }}=\bar{t}_{\text {outlet }}-\bar{t}_{\text {inlet }} \\
& \sigma_{\text {bed }}^{2}=\sigma_{\text {outlet }}^{2}-\sigma_{\text {inlet }}^{2}
\end{aligned}
$$

Once $\bar{t}$ and $\sigma^{2}$ are known for the bed, it can be shown that Peclet number, $\mathrm{Pe}$, may be evaluated by the following expression:

$$
\mathrm{Pe}=\frac{2 \bar{t}^{2}}{\sigma^{2}}
$$




\subsubsection{Liquid Holdup and Film Thicloness:}

One of the promising features of rotating beds is their short residence time and low liquid holdup. The residence time distribution data provides some indication of liquid holdup.

The liquid holdup in rotating beds may also be estimated using rotating disk analysis. The velocity profiles and the film thickness of liquid film on a rotating disk, a problem related to the spin coating procedure in the semiconductor industry, have been studied by several investigators [33-37]. Methods such as polynomial approximation of velocity components and the momentum integral method have been used to describe the liquid flow on a rotating disk. One of the interesting results of the momentum integral method is the analogy of the asymptotic solution of film thickness for a rotating disk with that of gravity liquid film flow along an inclined plane [22]. The asymptotic solution of liquid flow along a rotating disk has the following form:

$$
\delta=\left[3 Q^{\prime} \mu_{1} / \omega^{2} T \rho_{1}\right]^{1 / 3}
$$

Here, $\delta$ is the liquid film thickness, $Q^{\prime}$ is the liquid flowrate per unit width, $\mu_{1}$ is the liquid viscosity, $\rho_{1}$ is the liquid density, $\omega$ is the speed of rotation, and $r$ is the radial distance. In the gravity flow the centrifugal acceleration, the $\omega^{2} r$ term, is replaced with the gravitational acceleration, $g$.

The liquid film thickness, derived from the above analysis of a rotating disk, may be related to the liquid holdup in a rotating bed by the following expression: 


$$
a_{w} \times \delta=h_{0} / \dot{V}
$$

Here, $h_{0}$ is the liquid holdup, $\dot{V}$ is the total packing volume, and $a_{w}$ is the wetted surface area of the packing. The value of $a_{w}$ may be estimated using the equation (27). The estimated values of the liquid holdup, using above analysis, may be compared with the values evaluated from RTD experiments.

\subsection{EXPERIMENTAL RESULTS AND DISCUSSION:}

Typical stimulus-response curves for a rotating bed are shown in Figure23 (see the Appendix for all the experimental runs). The output trace is noisier than the input, which is not surprising given that the output electrode has a trough shape and collects only a thin non-uniform liquid film thickness. The electrode responses do not reveal any indications of channeling (extra peak as a result of bypassing liquid) or stagnancy (long tail because of a stagnant region). It is believed that the appearance of shallow tails in some of the responses may not be suggestive of a stagnant region in the bed; rather, they are caused partly as a result of slow wash-off of the salt solution on the electrodes surfaces. Proper design of the electrodes (use of ieflon material) eliminated these problems to a great extent. The data was smoothed, and the mean residence time was calculated from equations (40) and (42).

\subsubsection{Gravitational Effect:}

Figure 24 shows the dependency of mean residence time on rotational speed. As expected, the mean residence time decreases as the rotational speed increases (at fixed liquid nowrate and no gas now). The mean residence time varied from 


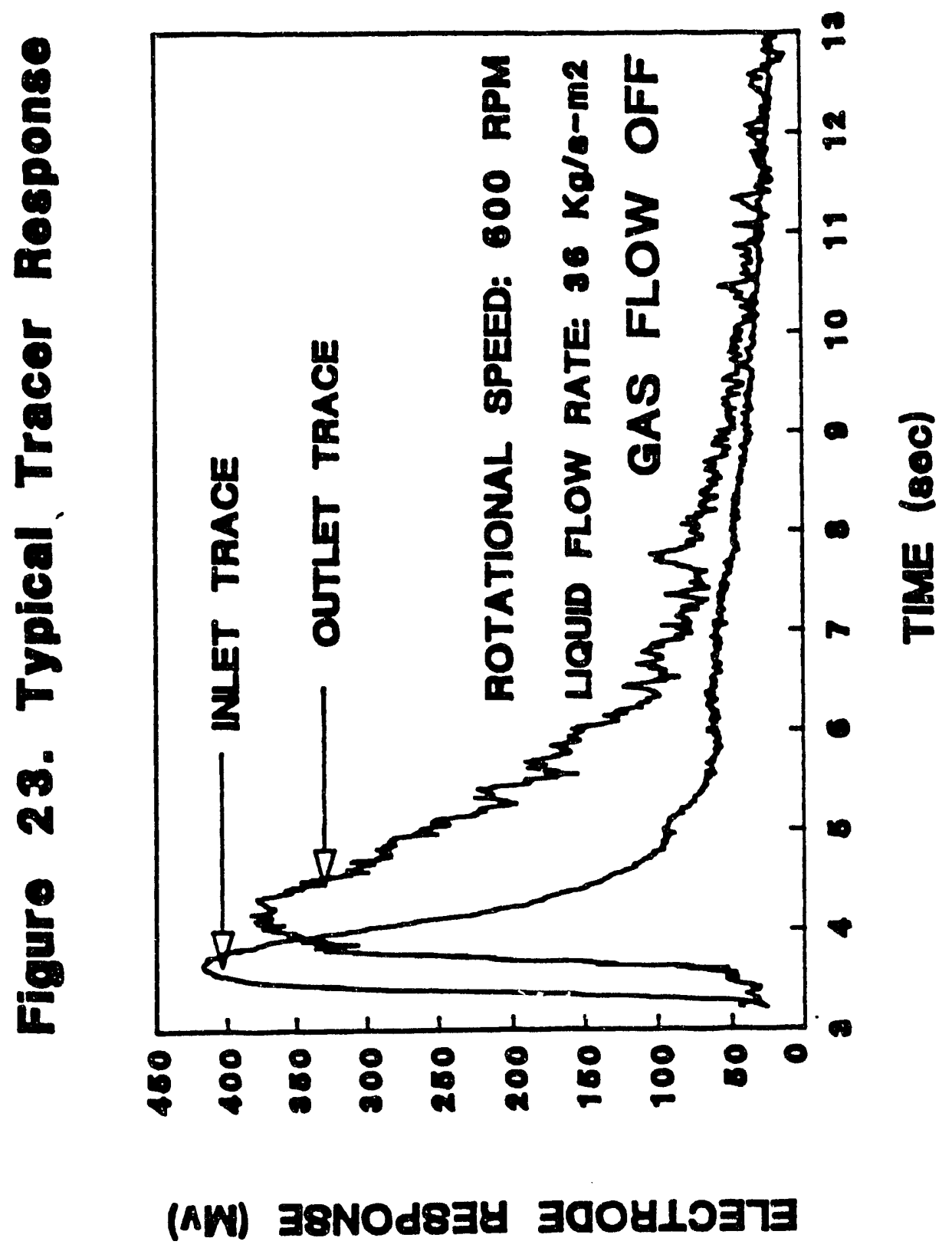




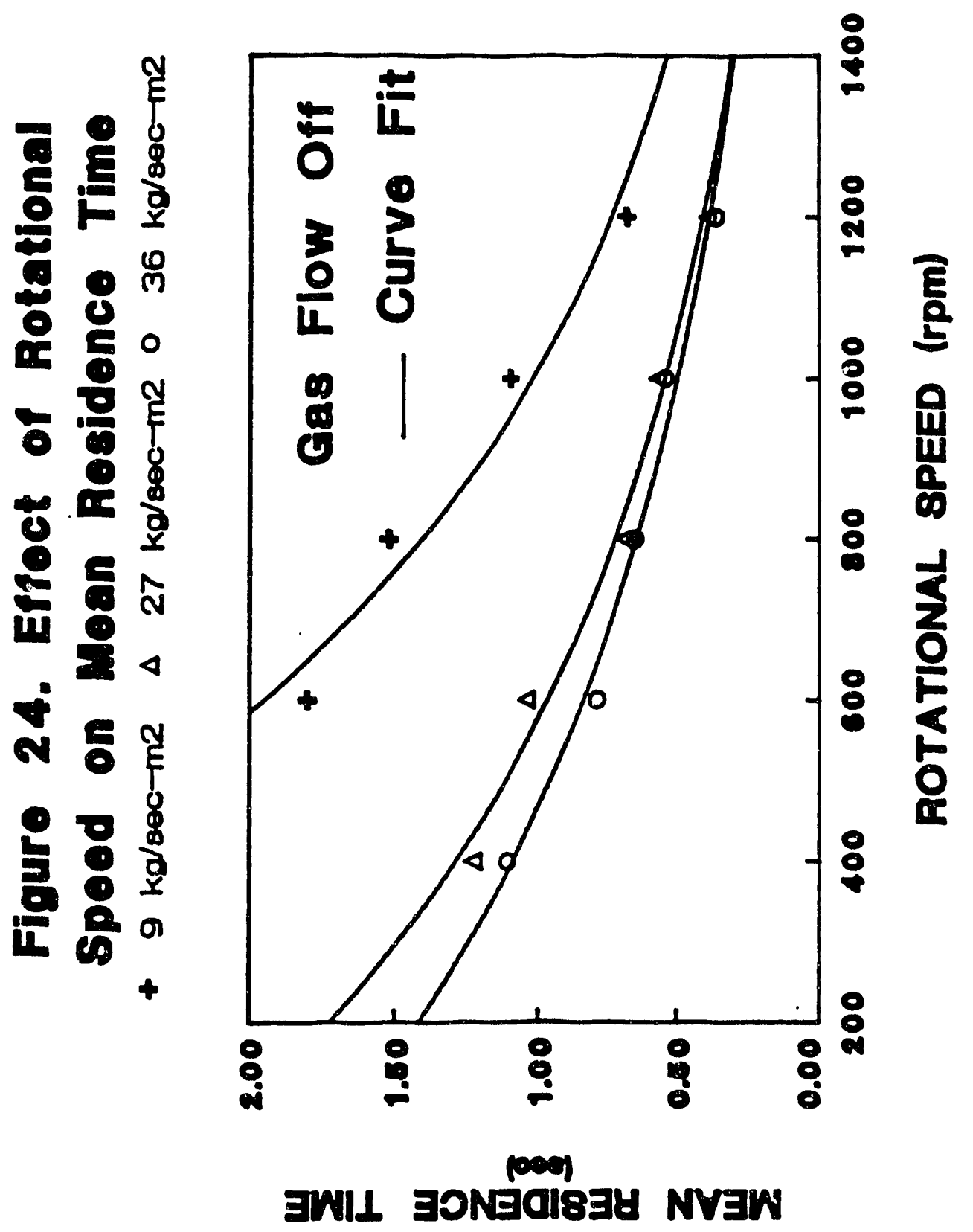


0.4 to 1.8 seconds. The outlet responses for high rotational speed were very noisy (particularly at 1000 and $1200 \mathrm{rpm}$ ). This is probably a result of more pronounced windage effects in higher rotational speeds, causing nonuniform liquid film over the trough shaped output electrode.

\subsubsection{Liquid and Gas Flowrates Effects:}

Figure 25 shows the dependency of mean residence time on liquid flowrate. At constant rotational speed and no gas flow, the mean residence time decreases as the liquid flowrate increases.

More tests were performed to investigate the effects of the gas flow on liquid residence time distribution. The results show that, statistically, the flowrate does not havesignificant effects on the distribution. However, the output response tends to be narrower in case of the gas flow-on, indicating shorter residence time. Figure 26 illustrates this effect.

\subsection{Comparison with Models:}

Equations (40) to (44) we:e used to evaluate Peclet number, Pe. Peclet numbers ranging from 0.1 to 3 were found, indicating highly dispersed flow conditions. Because of the large extent of backmixing (dispersion), as indicated by the low $\mathrm{Pe}$, it is unlikely that the assumptions and simplifications of the dispersion model will be satisfied [33] for the rotating bed analysis. The mixing patterns in rotating beds are too complex. However, attempts could be made to develop a mixing model for rotating beds based on the combined model. 


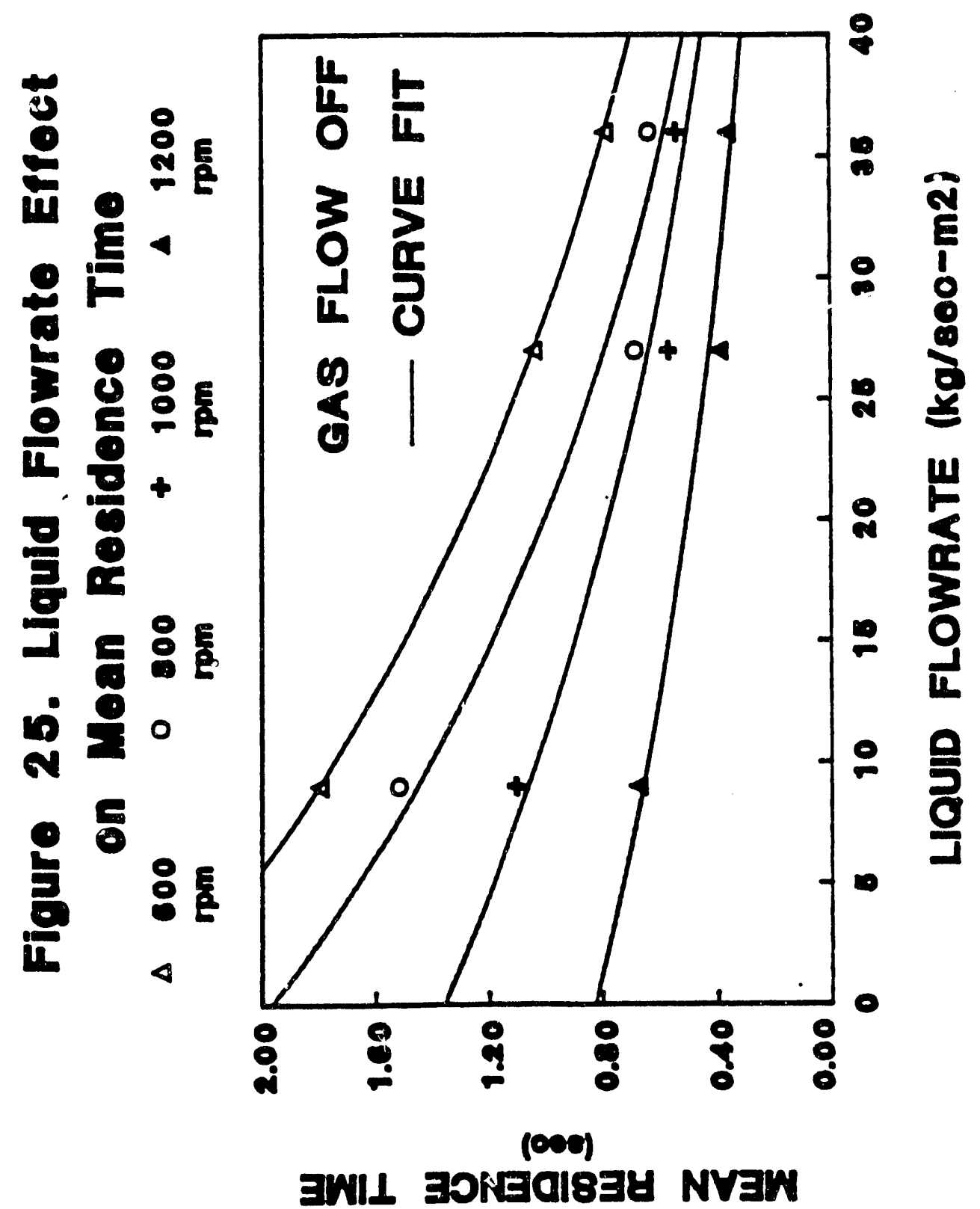




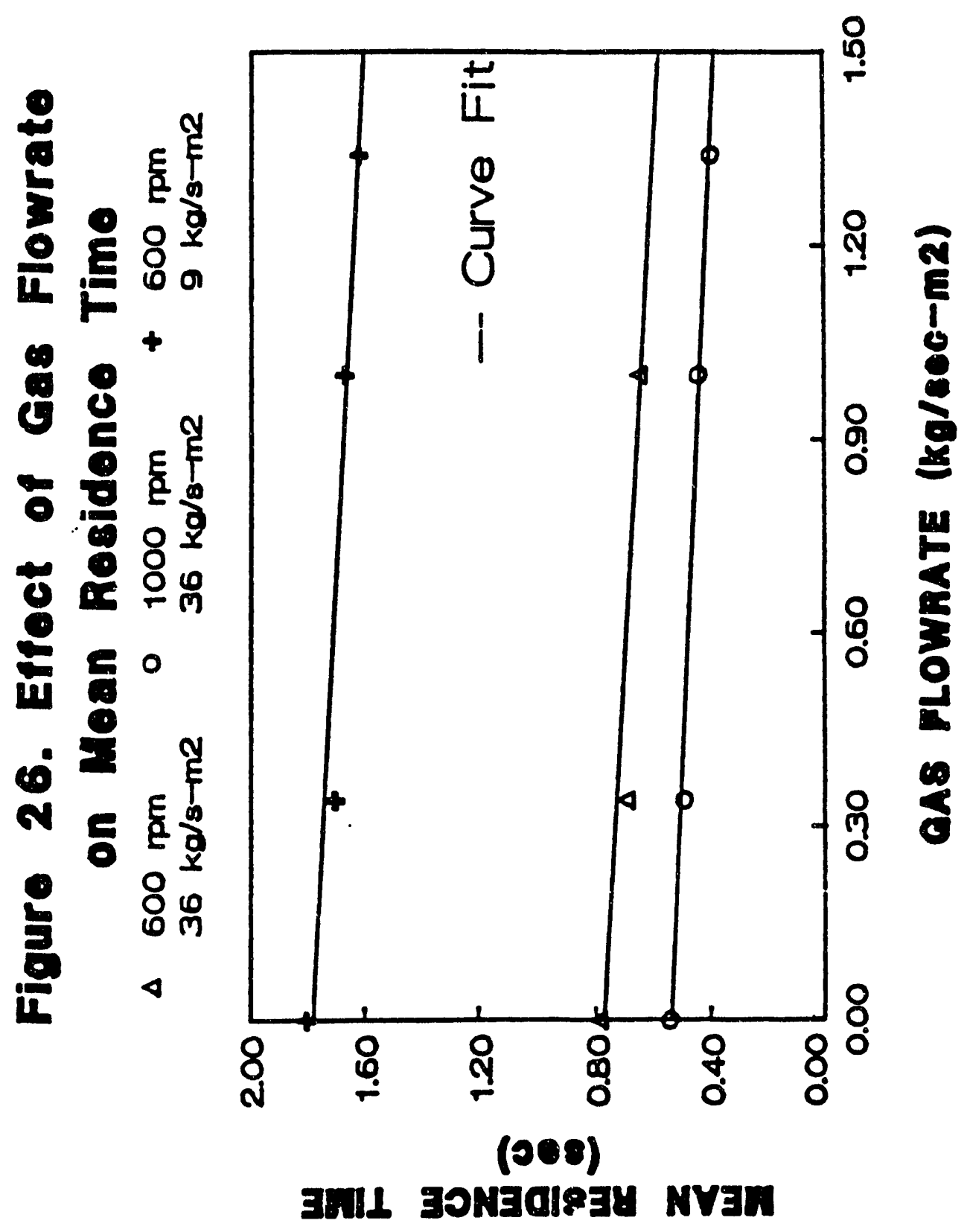


Equations (45) and (46) were used to estimate the liquid boldup. The residence time distribution data also provides some indication of liquid holdup. From the known liquid flowrate, the liquid holdup may be estimated the mean residence time multiplied by the flowrate gives the apparent holdup. These values are compared and plotted in Figure 27. The deviation at the higher speed (lower liquid film thickness) is because the asymptotic solution, equation (45), does not give a good prediction of liquid film thickness at high speed of rotation[14]. 


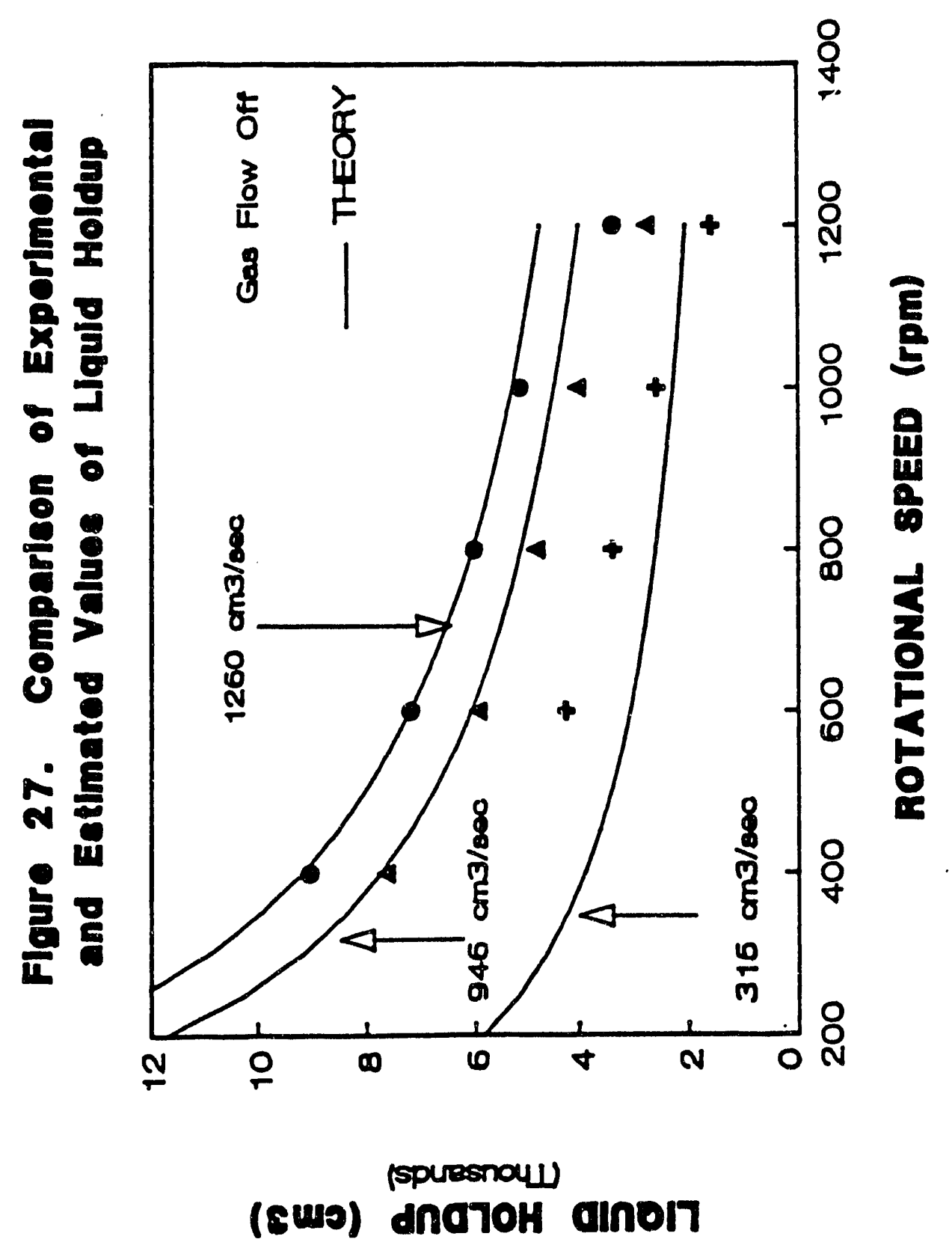




\section{CONCLUSIONS and RECOMMENDATIONS:}

In spite of some similar trends in pressure drop characteristics between a rotating packed bed and a conventional packed tower, the experimental results of the pressure drop indicate that they are some substantial differences in hydrodynamics behavior of gas-liquid in these two devices.

The total pressure drop is the sum of the pressure drop inside of the rotor and the pressure drop outside the rotor (between the rotor and the stationary housing). In this study, up to $10 \%$ of the total pressure drop is contributed by the pressure drop outside of the rotor. The agreement between the calculated values and experimental data for the pressure drop was within $\pm 20 \%$ error. It would also be very useful to measure the pressure at different positions along the housing to investigate the effects on the pressure drop measurements. The measurements may be improved if a five-hole Pitot tube is used to account for the static and dynamic pressure for the three-dimensional flow between the rotor and the hosing (making sure that the dynamic pressure component is not contributing to the total pressure drop measurements).

The experimentally measured height of a transfer unit decreases with the gravitational field with 0.30 to 0.4 power. This means that the volumetric mass transfer coefficient should rise with 0.6 to 0.7 power. This finding is in agreement with the data of Vivian et al [6] and within the range estimated by Dudukovic et al $[14,15]$. The height of a transfer unit, HTU, is found to be 13 to 30 times smaller in the rotating bed, as compared with that of conventional towers over the same range of liquid mass velocity. The magnitude of the end effects may be studied in 
more detail by estimating these effects in all the possible regions that contribute to the end effects. This may provide some information about the discrepancy observed in the measurements of volumetric mass transfer coefficients at different packing specific surface areas.

The predicted values of HTU, using the penetration theory modified for rotating beds, showed a reasonable agreement with the experimentally obtained values of HTU at varying operating conditions.

The required total power, in the range of the experimental operating conditions, was urjer 1 joule/gram of liquid. The largest contributor to power consumption is the acceleration of the liquid to the top speed of the rotor. The power consumption due to the bearing friction is proportional with the rotational speed. The introduction of the gas reduces the overall power required for rotating beds.

Mean residence times on order of 0.4 to 2 seconds were obtained for the rotating bed as the rotational speeds were varied fron 400 to $1200 \mathrm{rpm}$. The mean residence time decreases as the speed of rotation is increased, or the liquid flowrate is increased. The gas flowrate tends to reduce the liquid mean residence time at constant liquid flowrate and rotational speed. 


\section{NOMENCLATURE}

$a_{e}$

$a_{p}$

$a_{r}$

$a_{n}$

A

$\mathrm{B}$

C

C

$d_{p}$

$\mathscr{D}$ diffusion coefficient $\left(\mathrm{cm}^{2} / \mathrm{sec}\right)$

$f_{r} \quad$ friction factor in radial direction

$f_{t} \quad$ friction factor in tangential direction

g gravitational constant $\left(\mathrm{cm} / \mathrm{sec}^{2}\right)$

$\overline{\mathrm{G}}$ molar gas flowrate (gmole/sec)

b axial height of packing $(\mathrm{cm})$

$\mathrm{H}$ tower height $(\mathrm{cm})$

$\dot{\mathrm{H}} \quad$ Henry's constant $\left(\mathrm{atm} / \mathrm{gmole} / \mathrm{cm}^{3}\right)$

$b_{0} \quad$ liquid holdup $\left(\mathrm{cm}^{3}\right)$

HTU height of a transfer unit $(\mathrm{cm})$

I mcrnent of inertia $\left(\mathrm{g}-\mathrm{cm}^{2}\right)$

$k_{1} \quad$ liquid side mass transfer coefficient ( $\mathrm{cm} / \mathrm{sec}$ )

L superfacial liquid mass flowrate ( $\left.\mathrm{g} / \mathrm{sec}-\mathrm{cm}^{2}\right)$

$\overline{\mathrm{L}}$ molar liquid flowrate (gmole/sec) 
NTU number of transfer units

Q liquid volumetric flowrate $\left(\mathrm{cm}^{3} / \mathrm{sec}\right)$

$\mathrm{P} \quad$ pressure $(\mathrm{Pa})$

$\Delta \mathrm{P} \quad$ pressure drop $(\mathrm{Pa})$

I radial distance $(\mathrm{cm})$

$\mathbf{r}_{\mathbf{i}} \quad$ rotor inside diameter $(\mathrm{cm})$

$r_{0} \quad$ rotor outside diameter (cm)

$\mathrm{R} \quad$ disk radius (cm)

s

gap between the rotor and the stationary housing $(\mathrm{cm})$

S

empty tower cross section $\left(\mathrm{cm}^{2}\right)$

$\mathrm{t}+$ time $(\mathrm{sec})$

$\mathrm{T}$ torque (dyne-cm)

$\bar{t}$ mean residence time (sec)

U velocity in tangential direction $(\mathrm{cm} / \mathrm{sec})$

$\mathrm{V}$ velocity in radial direction $(\mathrm{cm} / \mathrm{sec})$

$\bar{V} \quad$ superficial velocity $(\mathrm{cm} / \mathrm{sec})$

$\hat{V} \quad$ packing volume $\left(\mathrm{cm}^{3}\right)$

$V_{i} \quad$ interfacial velocity $(\mathrm{cm} / \mathrm{sec})$

$x \quad$ axial direction $(\mathrm{cm})$

$\varangle \quad$ equilibrium concentration (gmole/ $\mathrm{cm}^{3}$ )

a liquid $\mathrm{CO}_{2}$ concentration in the rotor inlet (gmole $/ \mathrm{cm}^{3}$ )

$\sigma_{0}$ liquid $\mathrm{CO}_{2}$ concentration in the rotor outlet (gmole $/ \mathrm{cm}^{3}$ )

y $\mathrm{CO}_{2}$ concentration in the gas (gmole $/ \mathrm{cm}^{3}$ )

\# $\mathrm{CO}_{2}$ concentration in the gas inlet $\left(\mathrm{gmole} / \mathrm{cm}^{3}\right)$ 
2 axial direction

Z characteristic size of the packing $(\mathrm{cm})$

Greek Notations:

c packing void fraction

$\varphi_{S} \quad$ packing particle sphericity

$\rho_{1} \quad$ liquid density $\left(\mathrm{g} / \mathrm{cm}^{3}\right)$

$\rho_{\mathrm{g}} \quad$ gas density $\left(\mathrm{g} / \mathrm{cm}^{3}\right)$

$H_{l} \quad$ liquid viscosity ( $\left.\mathrm{g} / \mathrm{cm} \mathrm{sec}\right)$

$\mu_{\mathrm{g}} \quad$ gas viscosity $(\mathrm{g} / \mathrm{cm} \mathrm{sec})$

$\tau \quad$ shear stress $(\mathrm{Pa})$

$\omega \quad$, rotational speed (rpm)

$\sigma \quad$ variance of residence time distribution (sec)

$\bar{\sigma} \quad$ liquid surface tension (dyne $/ \mathrm{cm}$ )

$\sigma_{c} \quad c r i t i c a l$ surface tension of packing (dyne $/ \mathrm{cm}$ )

$\delta \quad$ liquid film thickness $(\mathrm{cm})$

Dimensionless Groups:

Fr $\quad$ Froude number $=L^{2} a_{p} / \rho^{2} g$

Gr Grashof number $=Z^{3} \rho^{2} g / \mu^{2}$

Re Reynolds number $=\mathrm{L} / \mathrm{a}_{\mathrm{p}} \mathrm{H}$

$\mathrm{Re}_{1} \quad$ Reynolds number $=4 \mathrm{~L} / \mu \mathrm{a}_{\mathrm{e}}$

Sc Schmidt number $=\mu / \rho \mathscr{D}$

We Weber number $=L^{2} / \rho \bar{\sigma} a_{p}$ 


\section{REFERENCES}

1. Podbielniak, W.J., "Continuous Centrifugal Vapor-Liquid Contactor", U.S. Patent 3,233,880 (1966).

2. Podbielniak, W.J., "Continuous Centrifugal Dcodorizer" Patent, 3,336,01 (1967).

3. Todd, D.B., "Multistage Vapor-Liquid Contacting", U.S. Patent , 3,486, (1969).

4. Todd, D.B., and D.C. Maclean, "Centrifugal Vapor-Liquid Contacting", British Chemical Engineering, 14, (11) p.1565, (1969).

5. Chambers, H.H., R.C. Walls, Trans. Instn. Chem. Engrs., 32, p.596, (1954).

6. Vivian, J.E., P.L.T. Brian, and V.J. Krudonis, "The Influence of Gravitational Force on Gas Absorption in a Packed Column", AIChE J., 1088 (1965).

7. Ramshaw, C., and R.H. Mallinson, "Mass Transfer Process", U.S. Patent $4,283,255$ (1981).

8. Ramshaw, C., "HIGEE Distillation- An Example of Process Intensification", The Chemical Engineer, pp.13-14, Feb. 1983.

9. Wen, J.W., "Centrifugal Gas-Liquid Contact Apparatus", U.S. Patent $4,382,045$ (1983).

10. Wen, J.W., "Centrifugal Gas-Liquid Contact Apparatus", U.S. Patent $4,382,900$ (1983).

11. Mohr, R.J., "The Role of HIGEE Technology in Gas Processing", Report GPA meeting in Dallas, (1985).

12. Basta, N., "Facelift for Distillation", Chem. Eng. Prog., 14-16, March 2,

13. Munjal S., M.P. Dudukovic, and P. Ramachandran, "Mass Transfer in Rotating Packed Beds. Part I: Development of Gas-Liquid-Solid Muss Transfer Correlations", Submitted for publication to Chemical Engineering Science (1987). 
14. Munjal S., M.P. Dudukovic, and P. Ramachandran, "Mass Transfer in Rotating Packed Beds. Part II: Experimental Results and Comparison with Theory and Gravity Flow", Submitted for publication to Chemical Engineering Science (1987).

15. Munjal, S., M.P. Dudukovic, and P. Ramachandran, "Mass I ransfer in a Rotating Packed Bed with Countercurrent Gas-Liquid Flow", Presented at the annual AIChE meeting in Cbicago, November 10-15, (1

16. Acrivos, A, B.D. Babcock, and R.L. Pigford, Chem. Eng. Sci., 10, 112-12 (1959).

17. Reshotko, E., and U. Rohatgi, "Analysis of Laminar Flow Between Stationary and Rotating Disks with Inflow", NASA Report \# CR-2356 (1974).

18. Cooper, P., "Turbulent Boundary Layer on a Rotating Disk

Calculated with an Effective Viscosity", AIAA Journah 9, No. 2, pp. 255, Feb. 1971.

19. Jimbo, H., "Investigation of the Interaction of Windage and Leakage Phenomena in a Centrifugal Compressor, A.S.M.E., Paper number $56-\mathrm{A} 47$.

20. Schultz-Grumow, F., Der Reibungwiderstand Rotierender Scheiben in Gehausen, Z. Angew. Math. Mech., 15, 191-204 (1935).

21. Reshotko, E., and R.L. Rosenthal, "Laminar Flow Between Two Infinite Disks, One Rotating and the other Stationary", Israel Journal of Technology, 9, p. 93, March 1971.

22. Bird, R.B., W.E. Stewart, and E.N. Lightfoot, Transport Phenomena, John Wiley \& Sons, New York, p.37, p.537, (1960).

23. Teutsch, T., Chem-Ing Tech, 36, No. 5, pp 496-503, (1964)

24. Onda, K., H. Takeuchi, and Y. Koyama, "Effect of Packing Materials on Wetted Surface Area", Chem. Eng. Japan, 31, 126-34 (1967).

25. Himmelldau, D.M., K.B. Bischoff, Process Analusis and Simulation, Chp. 4, Wiley: New York, 1974.

26. Seinfled, J.H., L. Lapidas, Mathematical Methods in Chemical Engineering, Vol. 3, Prentice-Hall: Englewood Cliffs, New Jersey, 1974. 
27. Nauman, E.B., and B.A. Buffham, Mixing in Continuous Flow Svstem, Chp. 1, John Wiley \& Sons Inc., N.Y. 1983.

28. Himmelldau, D.M., K.B. Bischoff, Process Analvsis and Simulation, Chp. 4, Wiley, New York, 1974.

29. Seinfeld, J.H., and L. Lapidas, Mathematical Methods In Chemical Engineering, Vol. 3, Prentice-Hall, Englewood Cliffs, New Jersey 1974.

30. Wen, C.Y., and L.T. Fan, Models for Flow Sustems and and Chemical Reactors, Marcel Dekker, Inc., New York (1975).

31. Levenspiel, O., and K.B. Bischoff, Patterns of Flow in Chemical Process Vessels, Advanced Chem. Eng., T.B. Drew, ed., 4, pp. 95-198 (1963).

32. Levenspiel, O., Chemical Reaction Engineering, 2nd Ed., Chap. 9, John Wiley \& Sons, Inc., New York (1972).

33. Matsuba, I.,and K. Matsumoto, "Transient Behavior of Spin-Coated Resist Film Thickness Based on Invariance of Viscous Fluid Under Simila Transformation", IEEE Transaction on Electron Devices, ED-33, No. 9, 1986.

34. Willus, C.A., and B. Fitch, "Flow Patterns in a Disc

Centrifuge", Chem. Eng. Prog. 69, No. 9, p. 73, 1973. .

35. Matsumoto, S., K. Saito, and Y. Takashima, "Thickness of a Viscous Liquid Film on a Rotating Disk", J. Chem. Engrg. of Japan, 6, 503-507, 1973.

36. Emslie, A.G., F.T. Bonner, and L.G. Peck, "Flow of a Viscous Liquid on a Rotating Disk", J. Appl Phys., 29, 858, 1958.

37. Marshall, JR. W.R, and E. Seltzer, "Principles of Spray Drying", Chem. Prog., 46, 501-509, 1950. 


\section{APPENDIX}

Section 11.1 shows the stress analysis made for the foam metal packing. Section 11.2 shows the calculations for the shaft design. Section 11.3 presents the the pressure transducers specifications and the calibration curves for the liquid and gas orifices. Section 11.4 presents the representative results of the mass transfer experimental runs.

\subsection{Stress Analysis for Aluminum Foam Metal Packing:}

When a circular disk rotates, the inertia forces can induce stresses that may become very large at high speed.

Consider a rotating disk with inner radius $r_{i}$ and outer radius $r_{0}$, and a rotational speed of $\mathrm{N}$. The maximum values of radial and tangential stresses can be calculated using the following equations:

$$
\begin{aligned}
& \left(\sigma_{\mathrm{r}}\right)_{\max }=\frac{\rho \mathrm{V}^{2}}{\mathrm{~g}} \times \frac{3+\nu}{8}\left(1-\frac{\mathrm{r}_{\mathrm{i}}}{\mathrm{r}_{0}}\right)^{2} \\
& \left(\sigma_{\mathrm{t}}\right)_{\max }=\frac{\rho \mathrm{V}^{2}}{\mathrm{~g}} \times \frac{3+\nu}{4}\left(1+\frac{1-\nu}{3+\nu} \times\left(\frac{\mathrm{r}_{\mathrm{i}}}{\mathrm{r}_{0}}\right)^{2}\right)
\end{aligned}
$$

where,

$\rho \quad$ is the density of the aluminum foam metal

$\mathrm{V}$ is the linear velocity $=2 \pi \mathrm{Nr}_{0}$

$\nu \quad$ is the Poisson ratio $=0$ for the aluminum foam metal 
For the our bed specifications, at $2000 \mathrm{rpm}, \sigma_{\mathrm{r}}$ and $\sigma_{\mathrm{t}}$ were $5 \mathrm{psi}$ and $\mathrm{i} 3 \mathrm{psi}$, respectively.

The permissible tensile stress for the aluminum foam metal, as reported by the manufacturer, is $165 \mathrm{psi}$. Thus, the packing is strong enough to withstand the applied stress at $2000 \mathrm{rpm}$ (gravitational field of $1000 \mathrm{~g}$, based on outside radius)..

\subsection{Shaft Design:}

Shaft design requires two steps: 1 -establishing the minimum shaft diameter, 2-determining the natural frequency of vibration for the shaft.

The bending moment, $M$, and the Torque, $T$, can act simultaneously; these loads can be combined and resolved into shear stress and tensile stress that can act on the shaft:

$$
\begin{aligned}
& \text { Torque }=T=\frac{\text { Power }}{\text { Rotat i onal Speed }}=\frac{P}{N} \\
& \text { Bending Moment }=M=\frac{\text { Power } \times \text { Shaft Length }}{\text { Rot ationaI Speed } \times \text { Rotor Diameter }}=\frac{P \times L}{N \times D}
\end{aligned}
$$

The minimum shaft diameter will be the greatest of the two values calculated from the following equations to meet the skear stress or tensile stress, respectively:

$$
d_{s}=\left(\frac{16 \times \sqrt{T^{2}+M^{2}}}{\pi \sigma_{s}}\right)^{1 / 3}
$$




$$
d_{t}=\left(\frac{16\left[M+\sqrt{T^{2}+M^{2}}\right]}{\pi \sigma_{t}}\right)^{1 / 3}
$$

Here, $\sigma_{s}$ and $\sigma_{\imath}$ are the allowable shear and tensile stresses.

For known rotor specifications, required power, and rotational speed, the diameter of the shaft may be determined. However, this value of the shaft also must meet the requirement of the natural frequency analysis, given by the following expression:

$$
N_{c}=\frac{d^{2} F_{b}}{L \times \sqrt{w \times(L+S b})}
$$

where,

$$
\begin{array}{ll}
N_{c} & \text { natural frequency } \\
d & \text { shaft diameter } \\
w & \text { weight of the rotor } \\
L & \text { shaft length } \\
S_{b} & \text { distance between the bearings } \\
F_{\text {m }} & \text { material properties factor }
\end{array}
$$

The operating speed of the shaft must not exceed the value of $\mathrm{N}_{c}$ calculated from the expression. 


\subsection{Calibration of the Transducers and Orifices:}

To measure the pressure drop across the orifices, differential pressure sensors (SenSym, SCX Series) were used. The sensitivity for the sensors used for the orifices is $6 \mathrm{MV} / \mathrm{psi}$. The full-scale span is $90 \mathrm{MV}$. The recorded data for various experimental runs were in range of $0-60 \mathrm{MV}$ for the gas orifice and $0-9 \mathrm{MV}$ for the liquid orifice.

The calibration of the gas orifice was carried out using: $i$ ) the orifice equation, ii) a rotameter, iii) a pitot tube. The calibration curves for these units are presented in Figures A1, A2, and A3, respectively. The calibration curves for (i) and (ii) cases have the second order feature, and they predict similar flowrate values (within 3\%). The calibration curve for the pitot tube does not show a second order functionality which is characteristic for orifice meters and the predicted flowrates are off by more than $50 \%$ compared to those of (i) and (ii) cases. A least square fit was employed for the obtained data, and the error analysis shows an error band of $\pm 0.9 \mathrm{ft}^{3} / \mathrm{min}$. Considering $\pm 5 \%$ full scale accuracy for the rotameter, the measured flowrates are accurate within $6 \%$ to $7 \%$. For this to be true, we assume that pressure transducer's inaccuracy is pressure independent; the $5 \%$ full scale accuracy for the rotameter is flow independent, and the error propagation is linear and can be superposed. Figure $4 \mathrm{~A}$ shows the linearity of the pressure transducer response in regard to the differential pressure.

Figure A5 shows the calibration of the pressure sensor used to measure the pressure drop across the bed. A monometer was used to calibrate this pressure transducer. This sensor has a sensitivity of $18 \mathrm{MV} / \mathrm{psi}$, and it bas a full scale span 
of $18 \mathrm{MV}$. The liquid orifice was calibrated by weighing the liquid output from the rotor for the periods of 20 second to 30 seconds. Figure $A 6$ shows the calibration curve for the liquid orifice. 


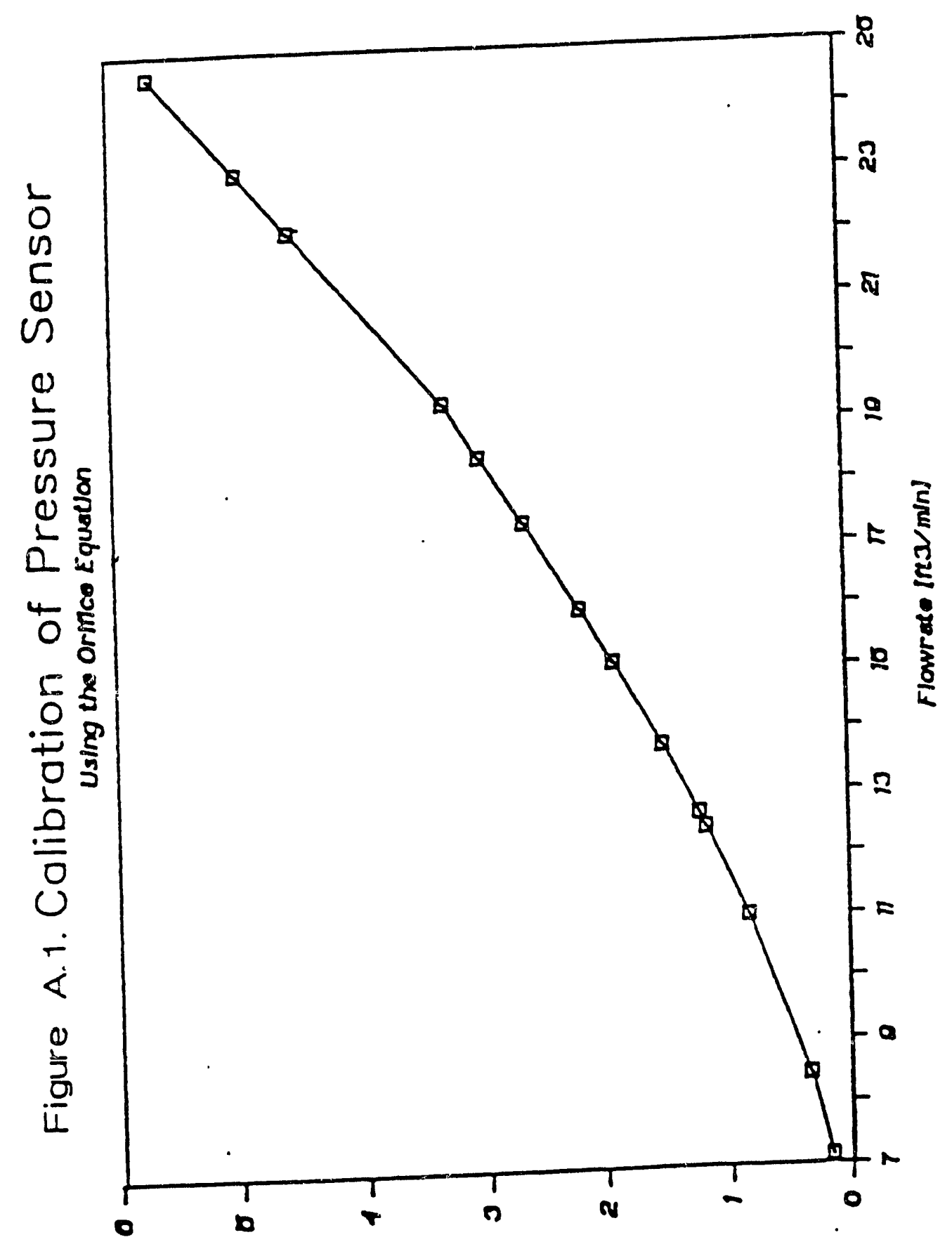

[HONIINU] OQRมด 


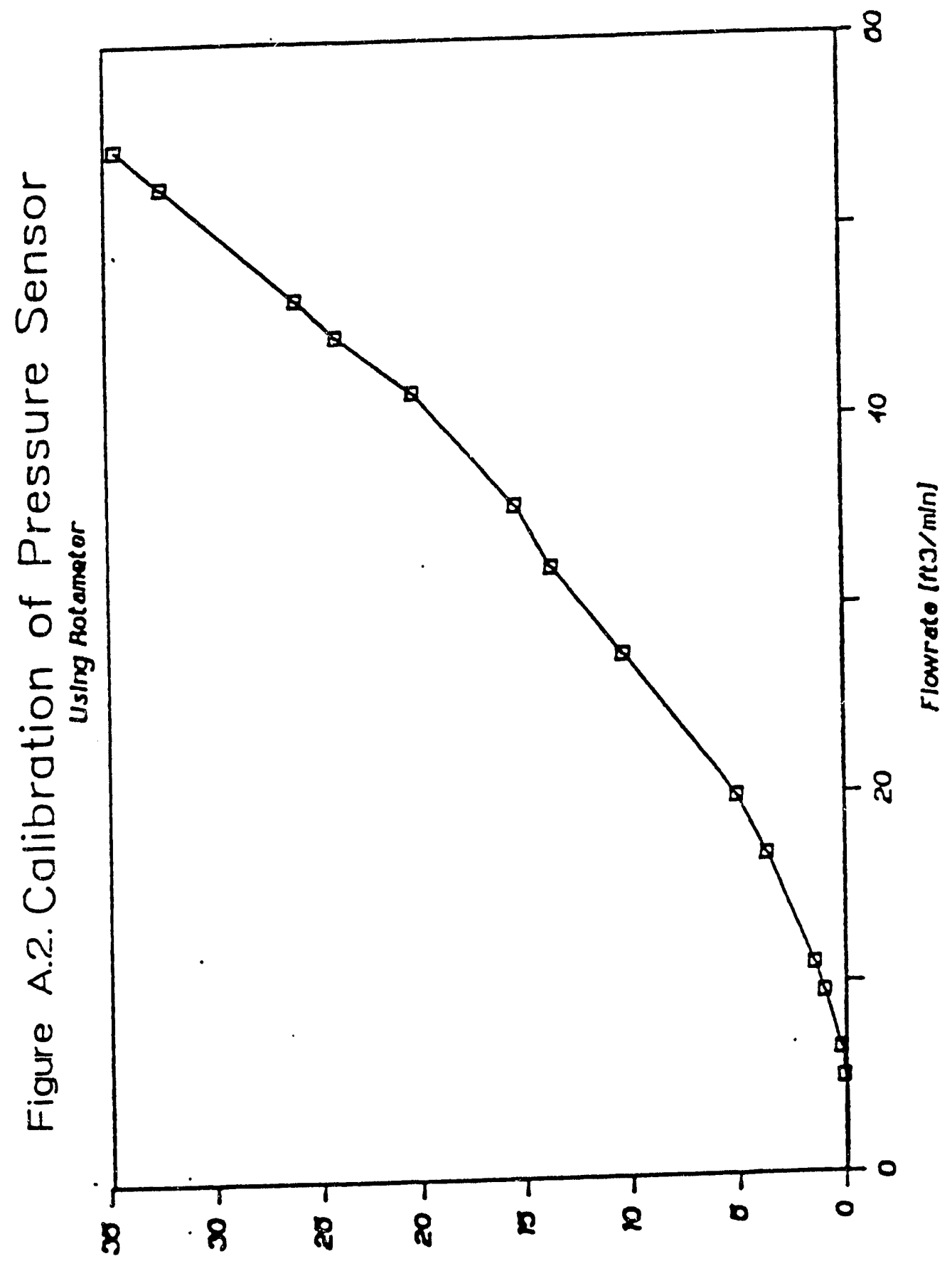

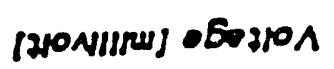




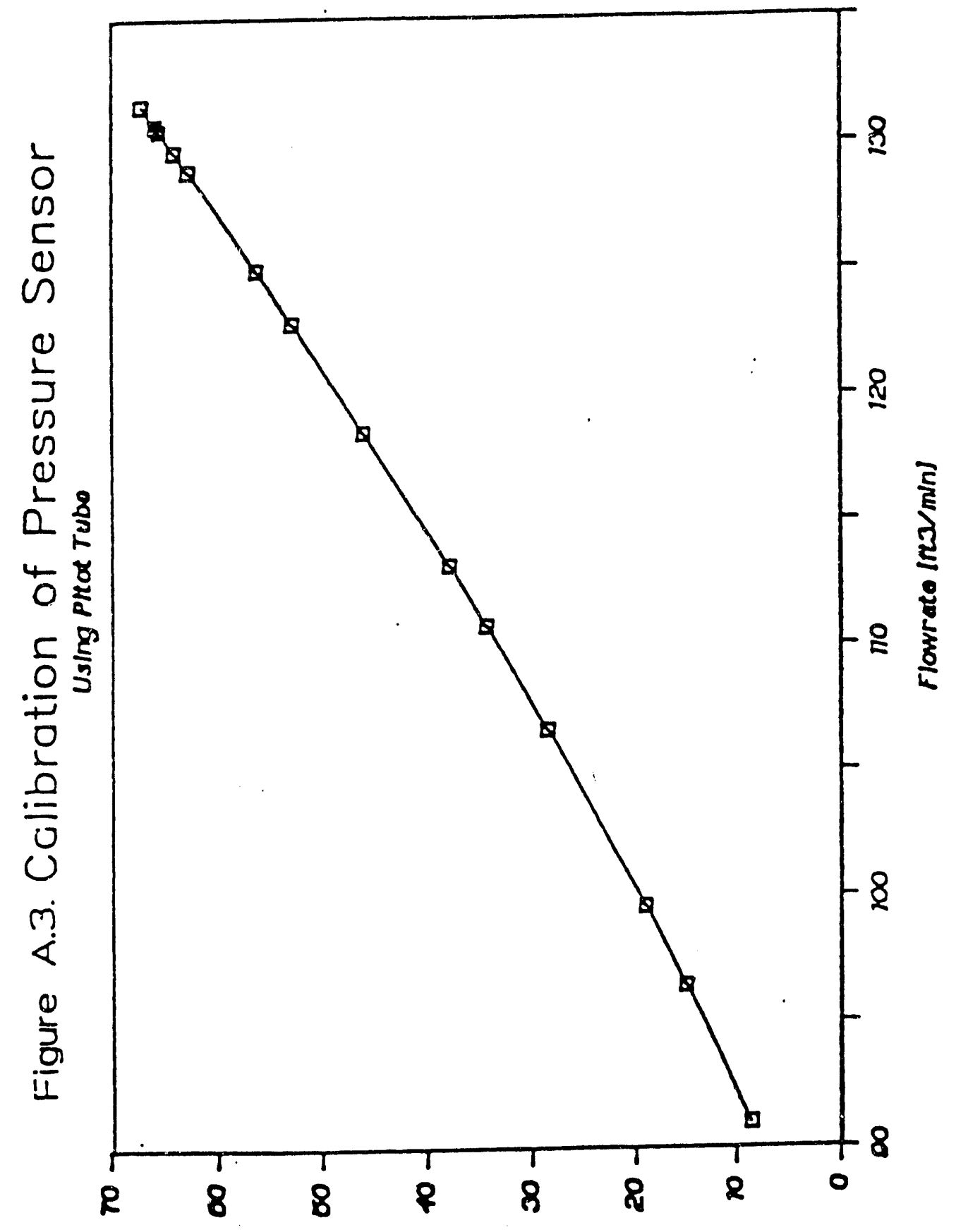

[20NIIR]] CBR 


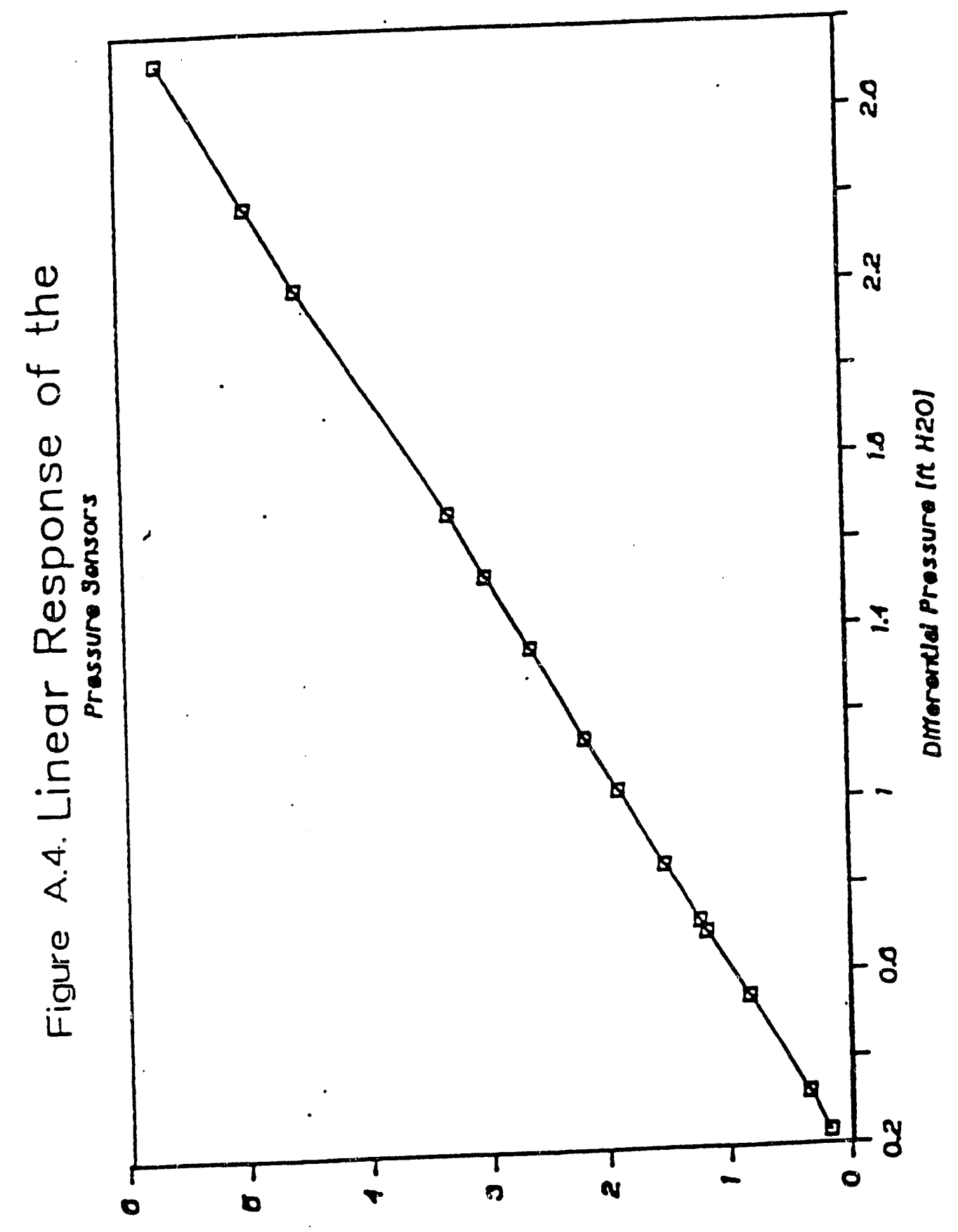

[HONIII)] -6\%นON 


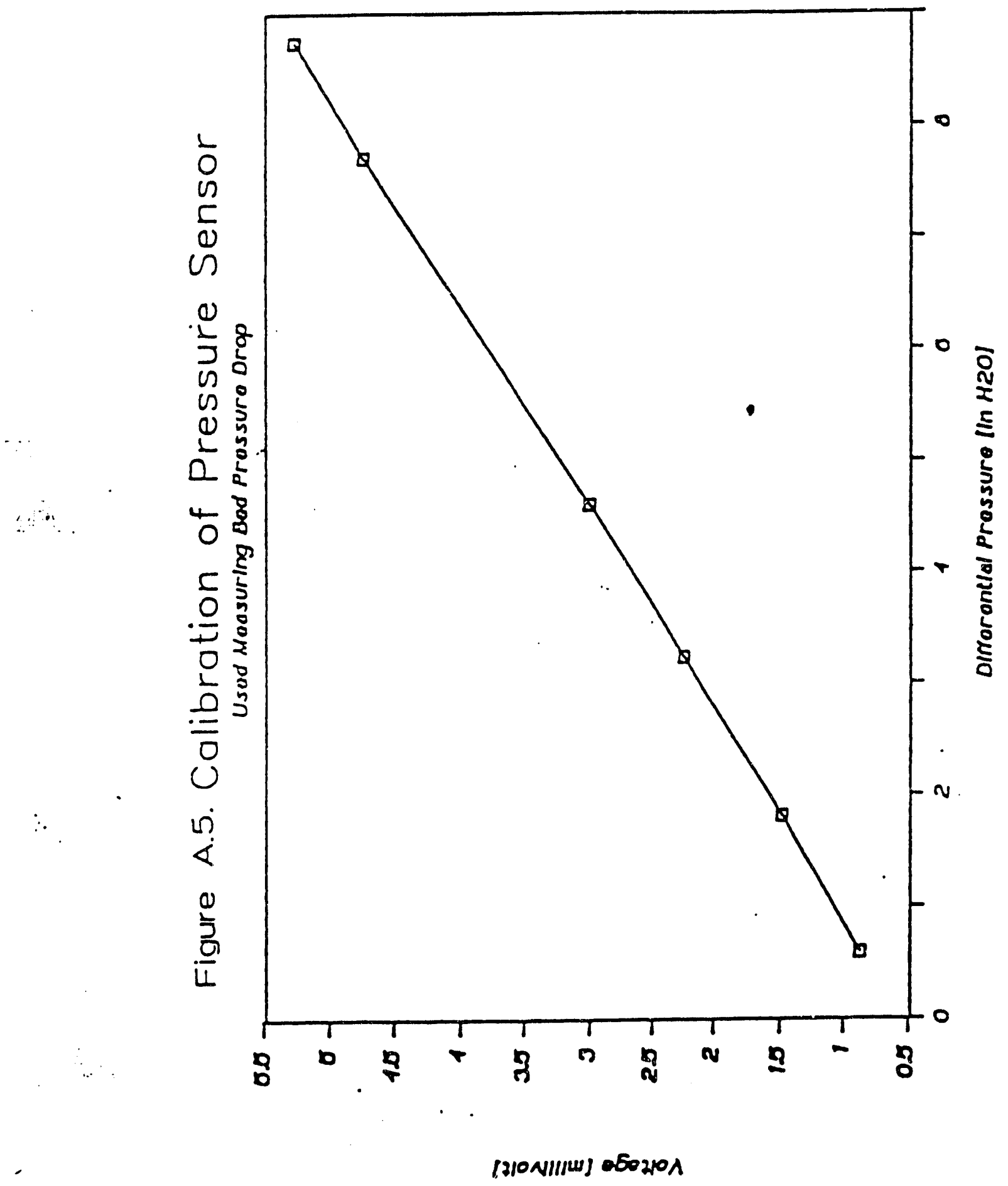




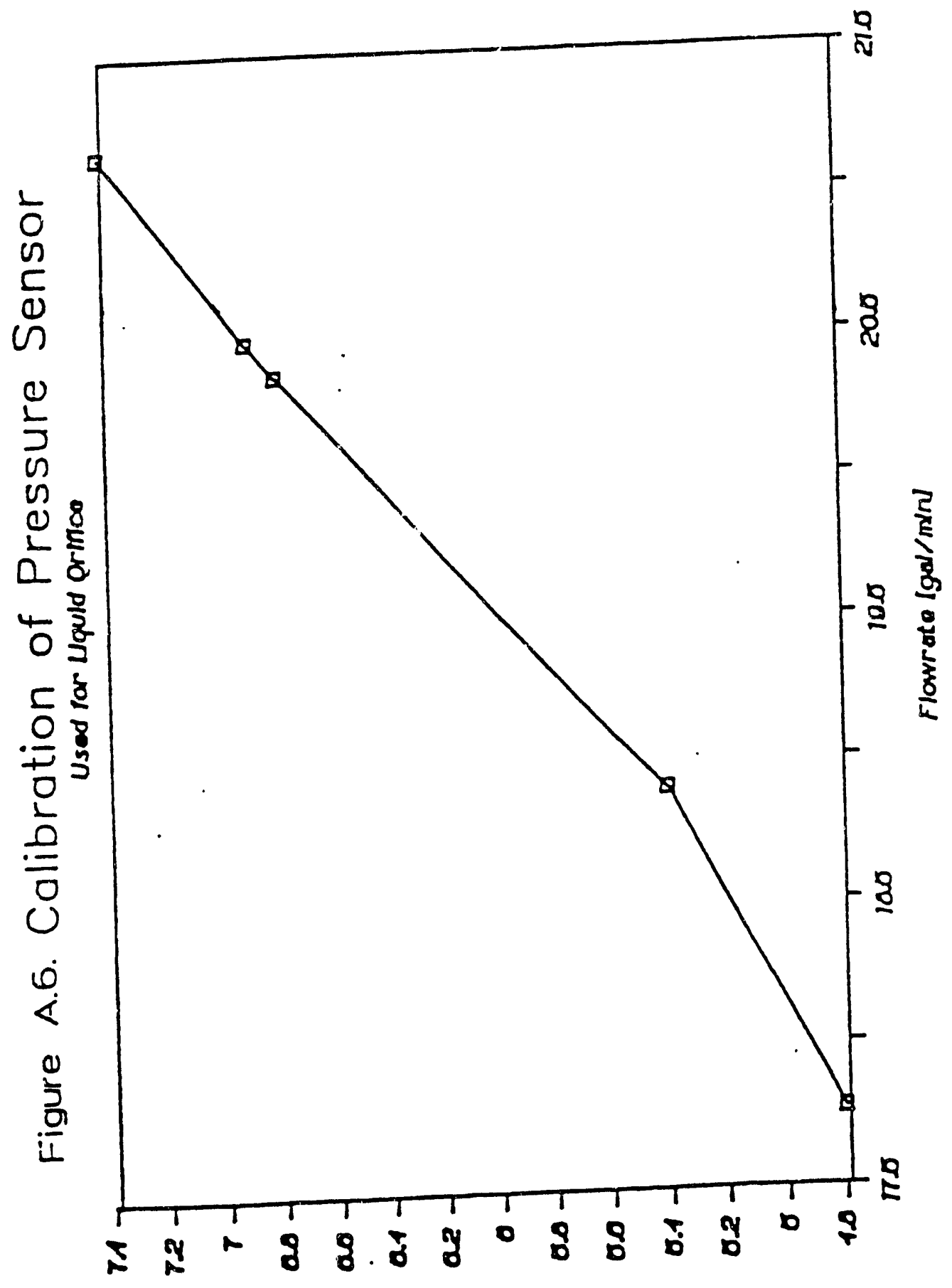

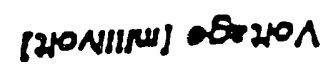




\subsection{Mass Transfer Experimental Runs:}

Desorption of carbon dioxide from water into air in rotating packed bed was studied. The carbon dioxide was dissolved in water by dissolving sodium bicarbonate in distilled water and then acidifying the solution to a pH of 5.0 (at $\mathrm{pH}$ between 4.8 and 5.2 all the carbonate and bicarbonate are converted to the CO2). Water containing discolved carbon dioxide was pumped from an overhead storage tank ( 760 liters capacity) through an orifice to the inner edge of the rotor.

The data from Dodds et al [AI] were employed for the solubility of $\mathrm{CO}_{2}$ in water. The accuracy of these data was not very significant in this investigation, since the liquid concentration in equilibrium with gas was quite small compared with the $\mathrm{CO}_{2}$ concentrations in the main body of the liquid. The equilibrium data was used to compute the hypothetical liquid concentration in equilibrium with the gas phase concentrations at the inlet and outlet of the rotor. In a few cases, gas analyses were taken for the purpose of material balance calculations, as a test of the accuracy of the data. The gas-phase concentration in the entering air was found by Gas Chromatograph to be about $5.5 \times 10^{-4}$ atmospheres, about twice that of the ordinary atmosphere $\left(3.3 \times 10^{-4}\right)$. 


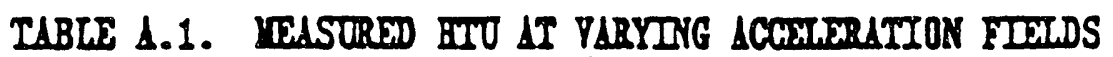
FOR $1476 \mathrm{n}^{2} / \mathrm{n}^{3}$ PACOMTiC

\begin{tabular}{|c|c|}
\hline LIQUID FLOYRATES: $36 \mathrm{Ig} / \mathrm{s}-\mathrm{m}^{2}$, GAS FLOTRATES: $1.0 \mathrm{Ig} / \mathrm{s}-\mathrm{m}^{2}$ \\
\hline BTO (cm) & $\mathrm{s}^{\prime} \mathrm{s}$ \\
\hline 3.76 & 50 \\
3.76 & 50 \\
3.80 & 50 \\
3.70 & 50 \\
\hline 2.74 & 100 \\
2.80 & 100 \\
3.30 & 100 \\
\hline 2.40 & 182 \\
2.25 & 182 \\
2.70 & 182 \\
\hline 2.00 & 272 \\
2.00 & 272 \\
\hline
\end{tabular}




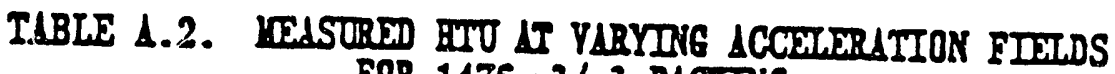
FOR $1476 \otimes^{2} / \mathrm{n}^{3}$ PLCONVG

\begin{tabular}{|c|c|}
\hline LIQUTD FLORRITE :18.0 $\mathrm{gg} / \mathrm{s}-\mathbf{s}^{2}$, GAS FLOYRITE: $1.0 \mathrm{Kg} / \mathrm{s}-\mathrm{m}^{2}$ \\
\hline ITO (cm) & $\mathrm{g}^{\prime} \mathrm{s}$ \\
\hline 2.98 & 50 \\
3.25 & 50 \\
3.00 & 50 \\
3.10 & 50 \\
2.98 & 50 \\
\hline 2.25 & 100 \\
2.11 & 100 \\
2.25 & 100 \\
2.36 & 100 \\
\hline 1.95 & 182 \\
1.98 & 182 \\
1.95 & .182 \\
2.00 & 182 \\
\hline 1.80 & 272 \\
1.79 & 272 \\
1.80 & 272 \\
\hline
\end{tabular}


TABLE 1.3. MTSASORID HIO IT YARYTYG ACCRTERATION FTRIDS FOR $2952 \mathrm{~m}^{2} / \mathrm{m}^{3}$ PACXING

\begin{tabular}{|c|c|}
\hline LIQUID FLOKRTE: $36 \mathrm{Ig} / \mathrm{s}-\mathrm{m}^{2}$ & , GAS FLOKRATE $: 1.0 \mathrm{gg} / \mathrm{s}-\mathrm{m}^{2}$ \\
\hline BTO (cm) & $\mathrm{g}^{\prime} \mathrm{s}$ \\
\hline 2.65 & 50 \\
2.71 & 50 \\
2.52 & 50 \\
2.22 & 50 \\
2.28 & 50 \\
2.28 & 50 \\
2.28 & 50 \\
\hline 1.94 & 100 \\
1.92 & 100 \\
2.07 & 100 \\
2.01 & 100 \\
1.83 & 100 \\
2.07 & 100 \\
\hline 1.70 & 182 \\
1.67 & 182 \\
1.70 & 182 \\
1.64 & 182 \\
\hline 1.40 & 272 \\
1.21 & 272 \\
1.22 & 272 \\
\hline
\end{tabular}




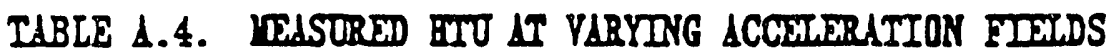
FOR $656 \mathrm{~m}^{2} / \mathrm{m}^{3}$ PACXNG

\begin{tabular}{|c|c|}
\hline LIQUID FLOKRATE: $36 \mathrm{Ig} / \mathrm{s}-\mathbf{n}^{2}$, GAS FLOVRATE $: 1.0 \mathrm{Ig} / \mathrm{s}-\mathrm{m}^{2}$ \\
\hline BTU (cm) & $\mathrm{g}^{\prime} \mathrm{s}$ \\
\hline 2.74 & 50 \\
2.74 & 50 \\
2.67 & 50 \\
2.74 & 50 \\
2.82 & 50 \\
2.74 & 50 \\
\hline 2.20 & 100 \\
2.20 & 100 \\
\hline 2.01 & 182 \\
2.00 & 182 \\
2.06 & 182 \\
\hline 1.95 & 272 \\
1.92 & 272 \\
1.86 & 272 \\
\hline
\end{tabular}


11.5. Residence Time Distribution Experimental Runs:

The normalized electrode responses for varying liquid and vapor loading, and rotational speed are presented in Figures 7A to 28A. The $\mathrm{Y}$-axis represents the electrode response in millivolts, while the $\mathrm{X}$-axis is time in seconds. The solid lines, drawn through the noisy part of the curves, indicates the effort made to smooth the data. A digitizer was used to transfer the smoothed points to a personal computer for the data analysis. 


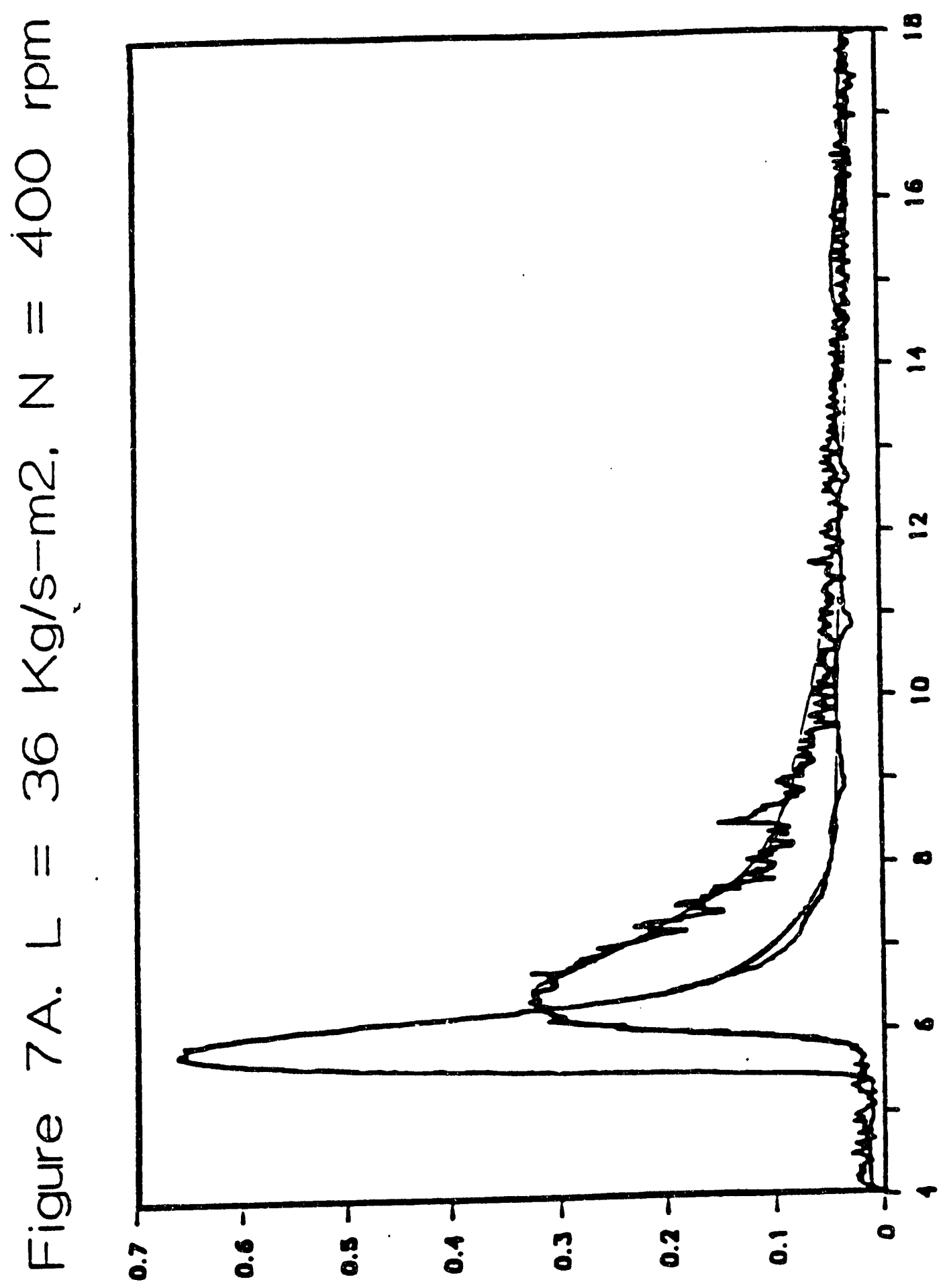




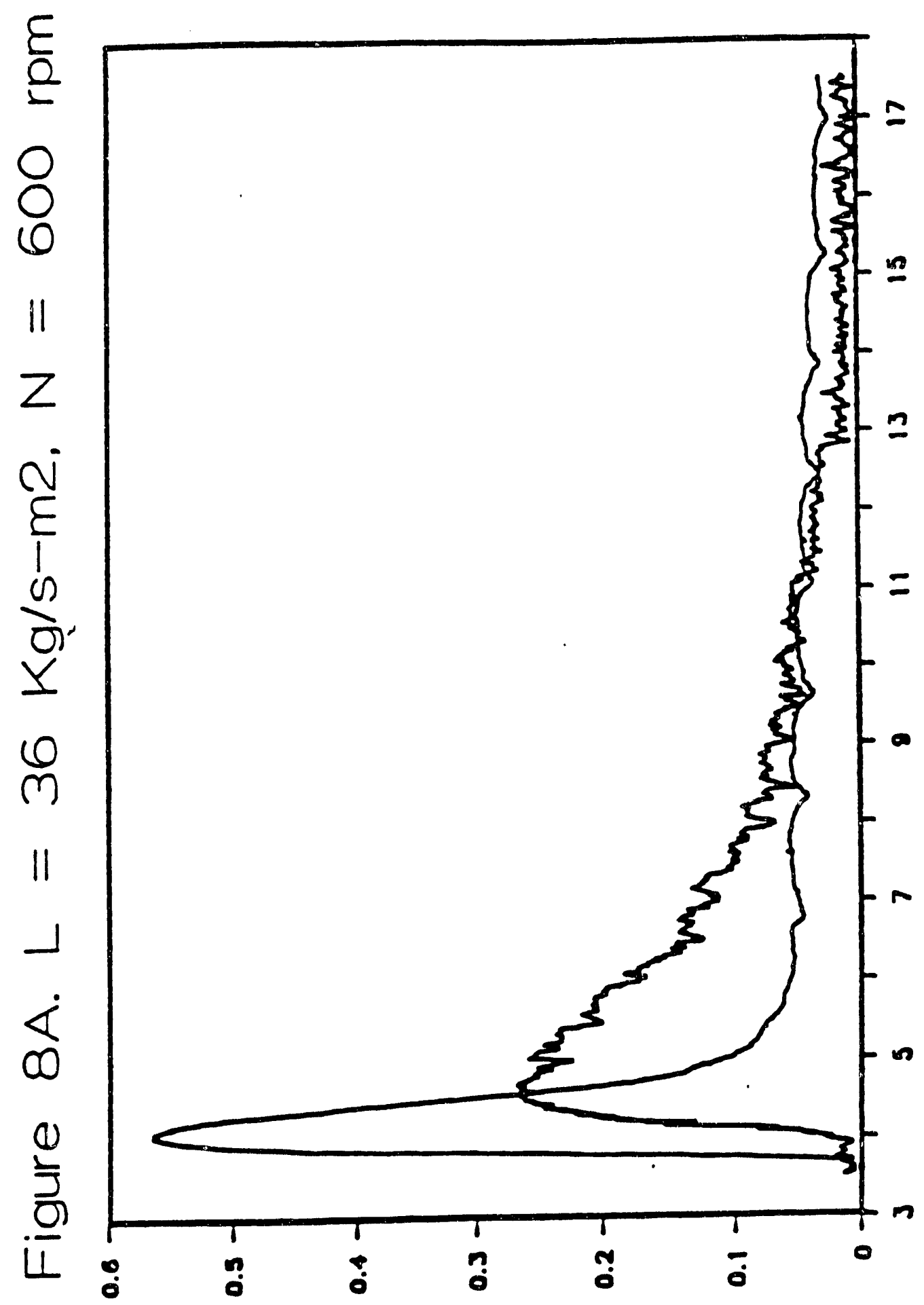




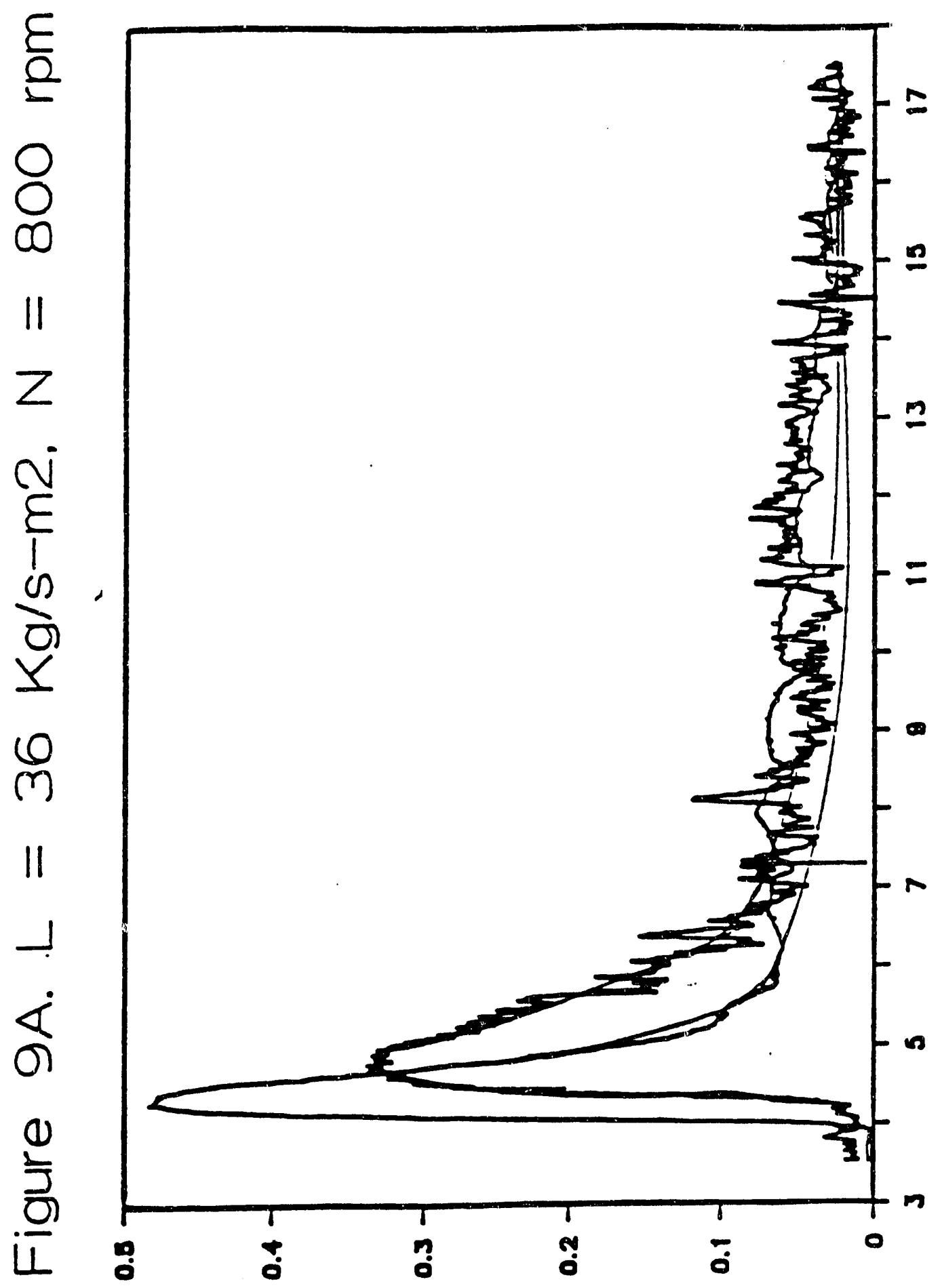




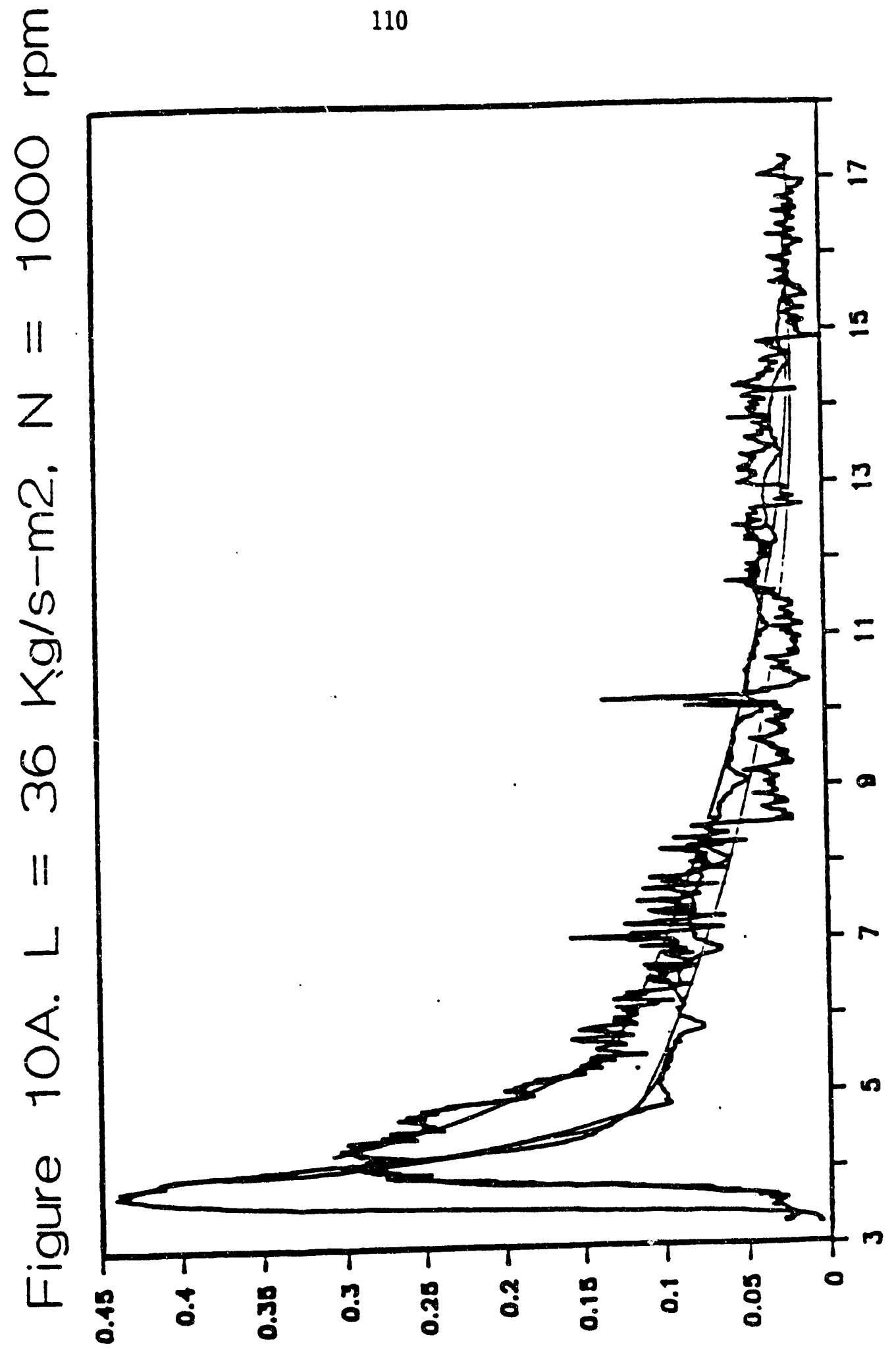




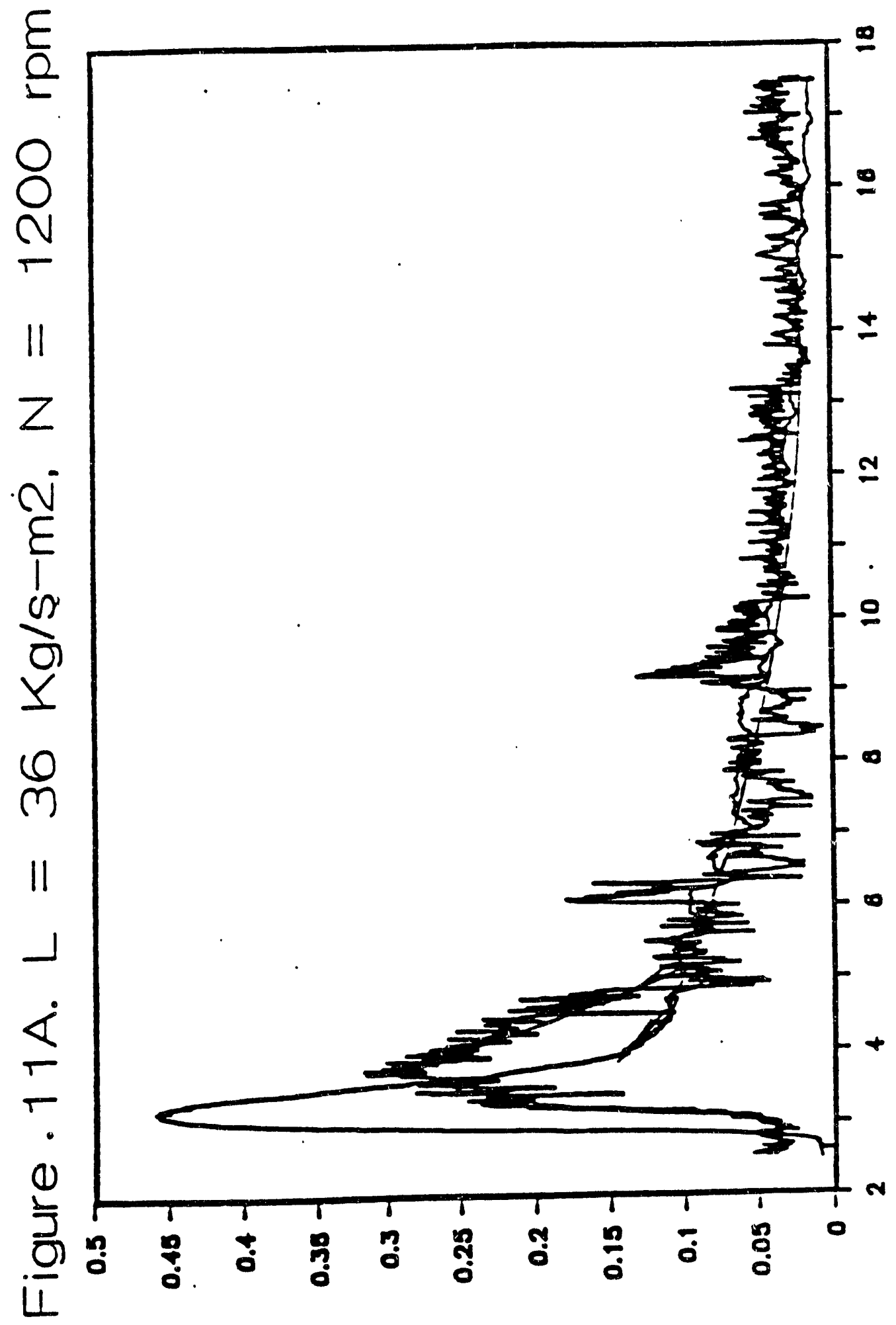




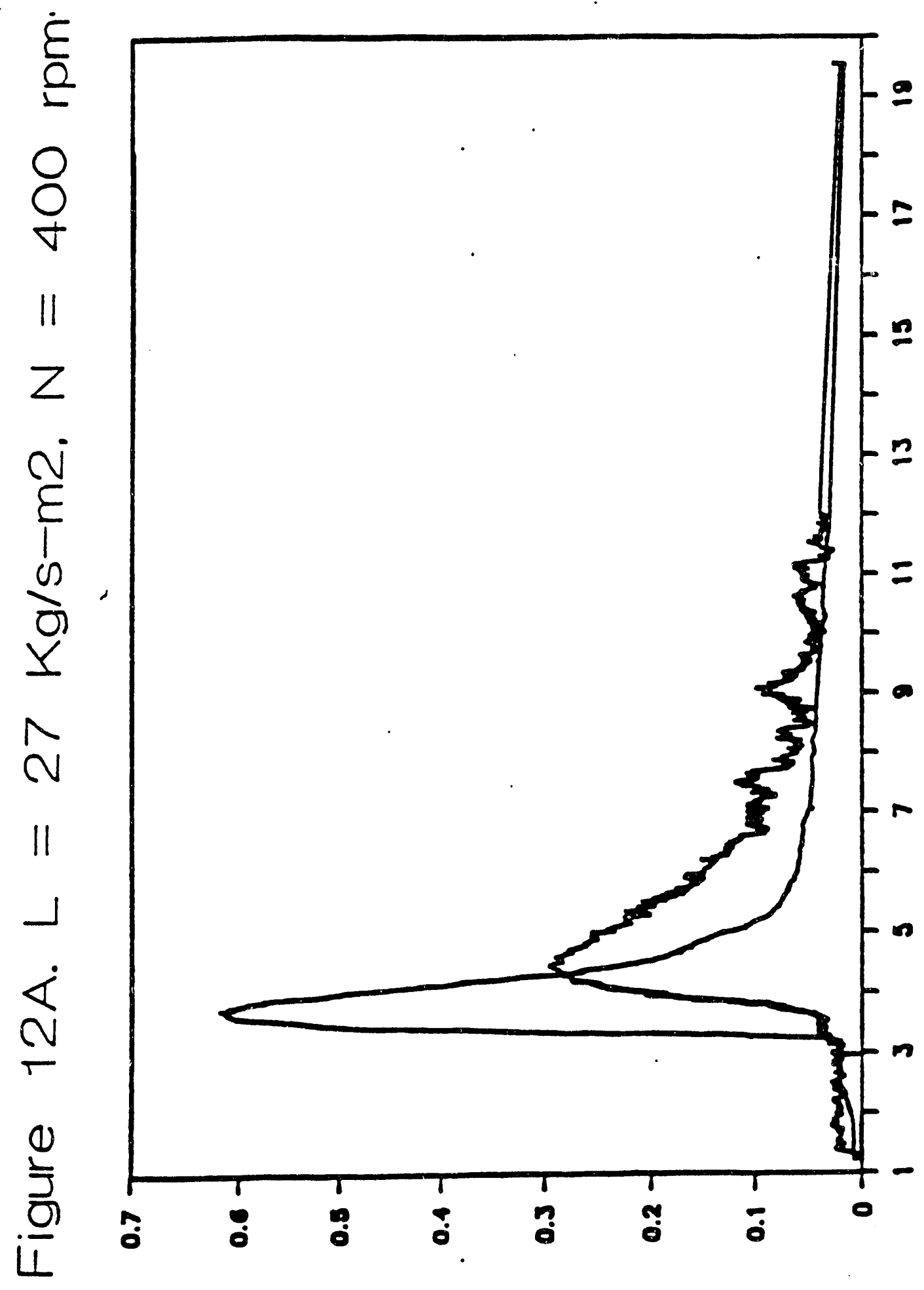




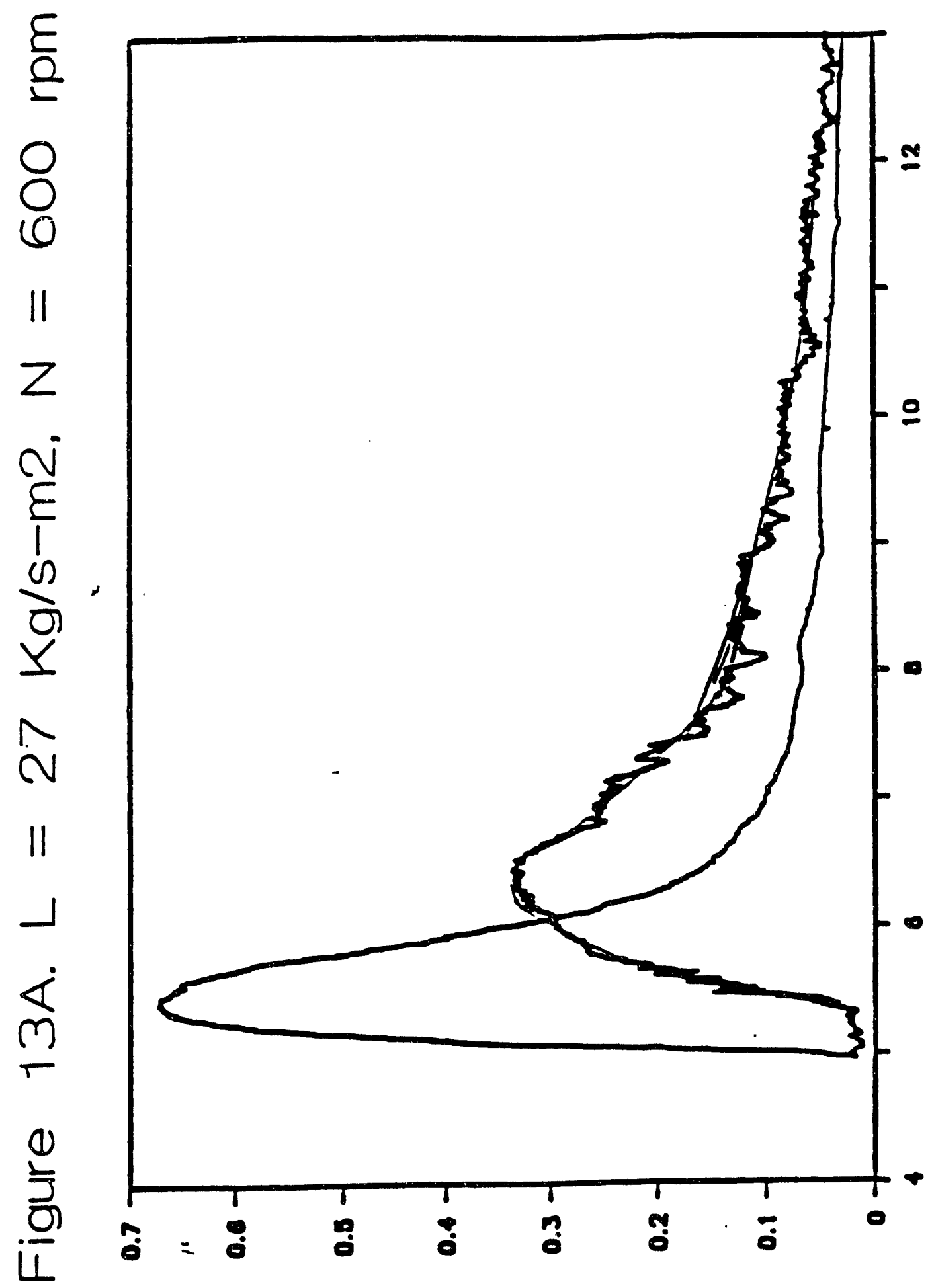




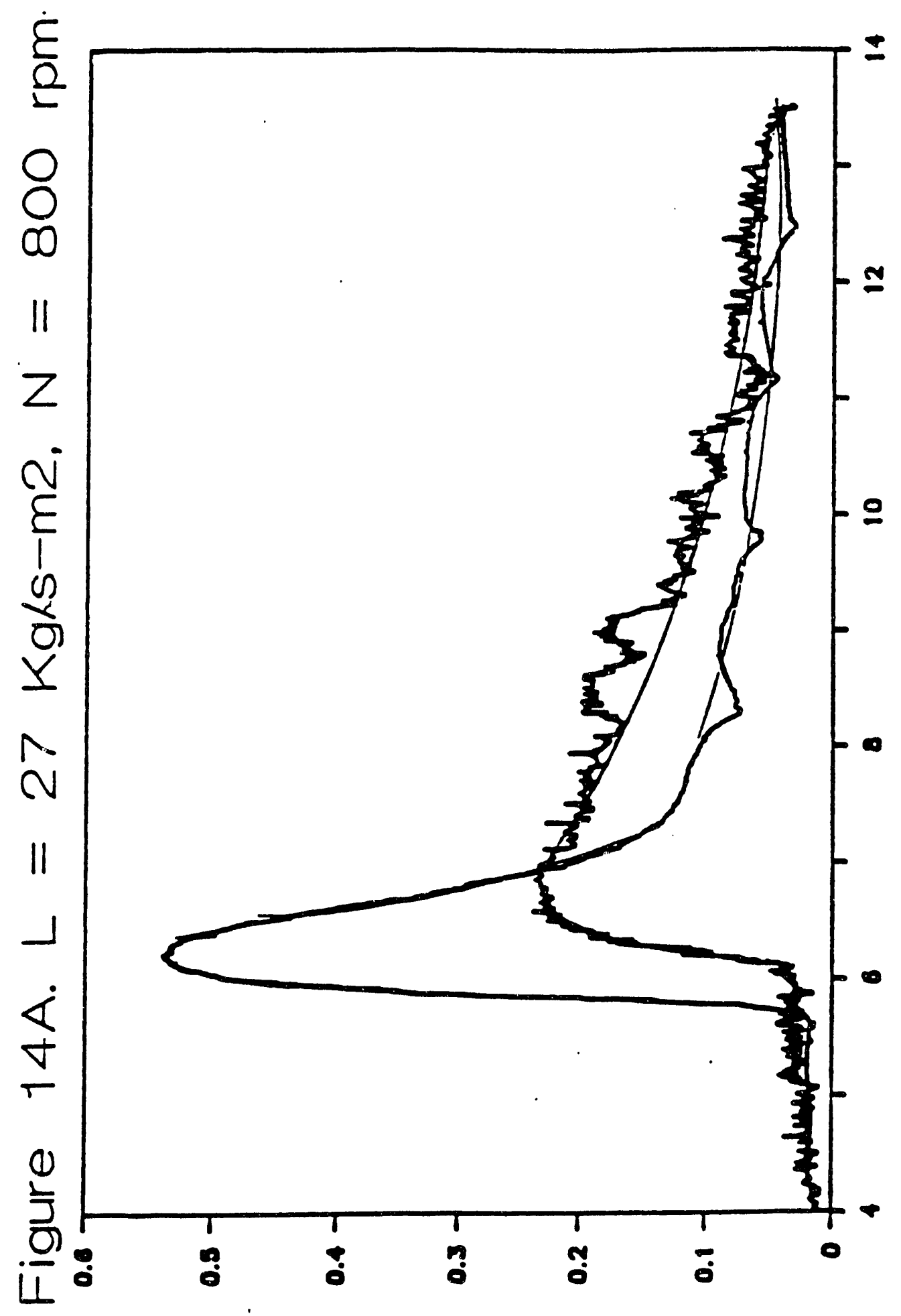




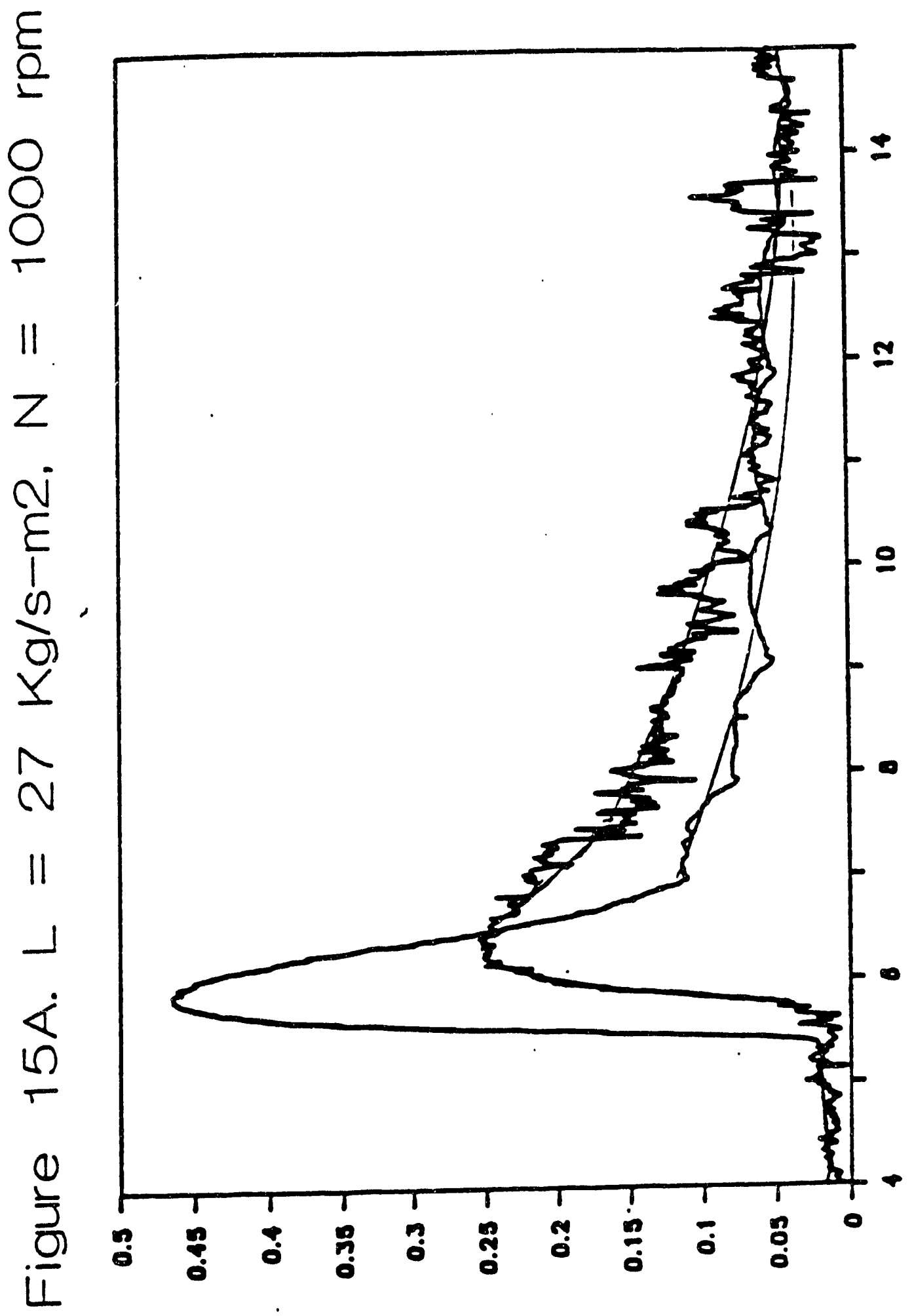




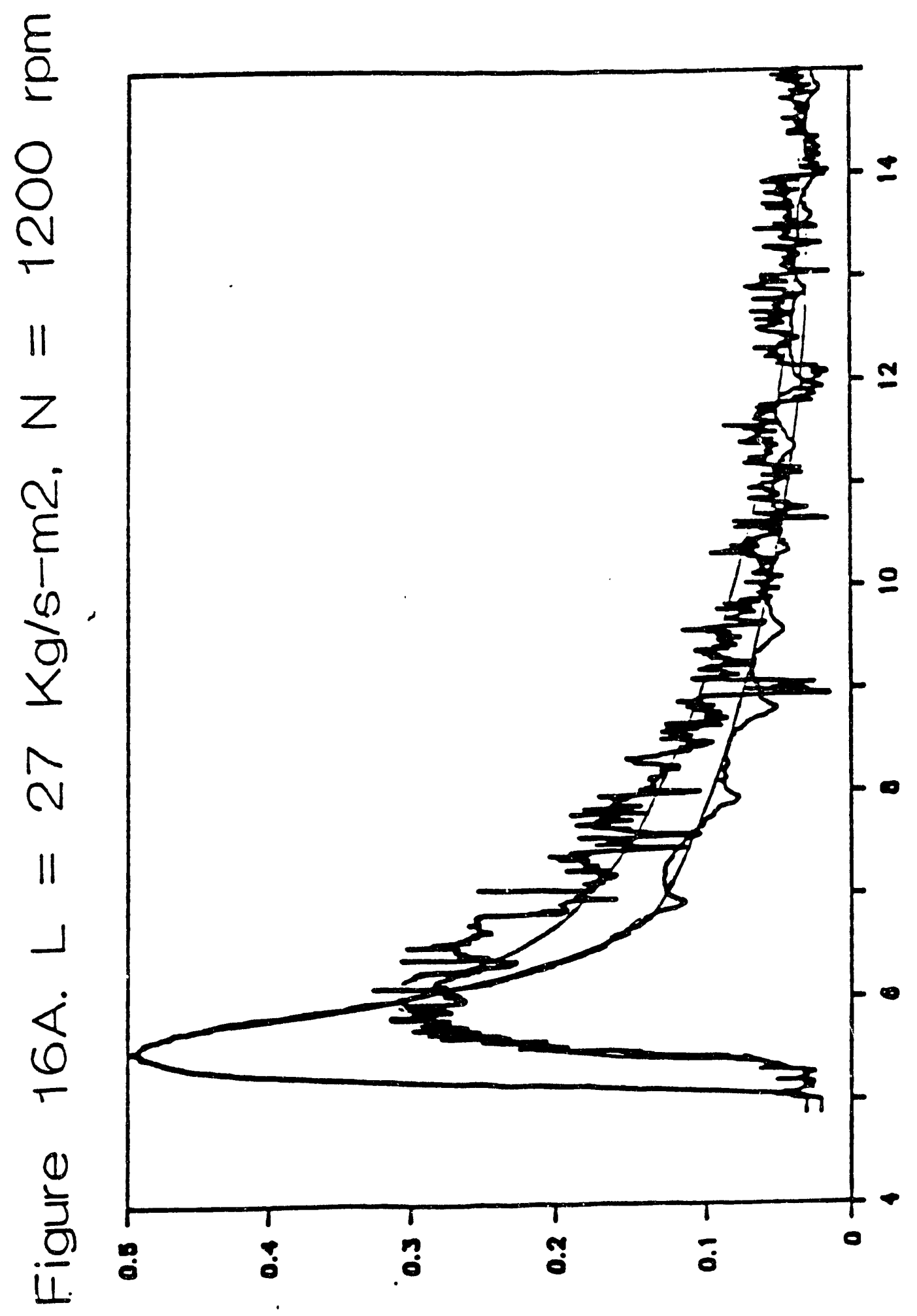




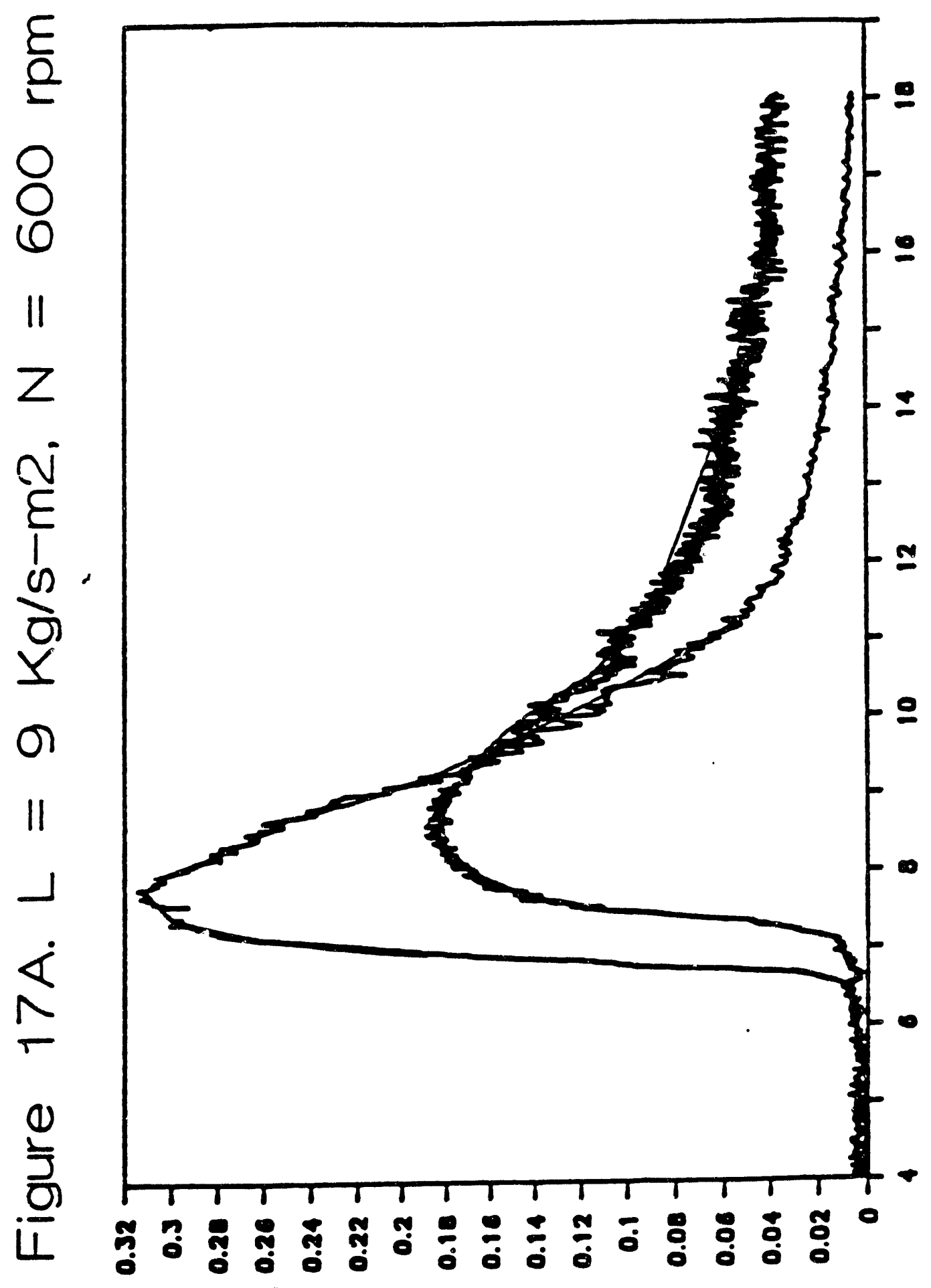




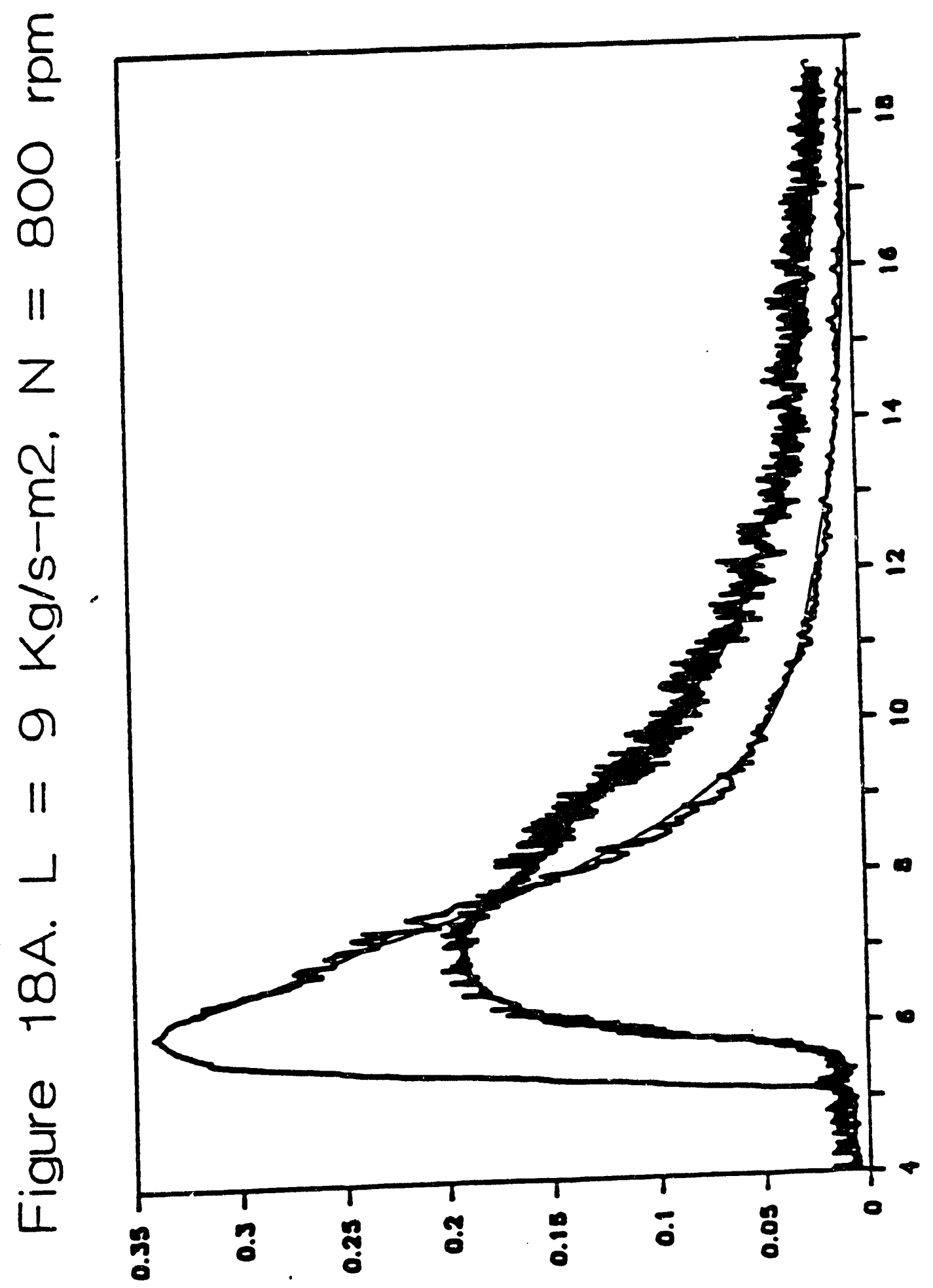




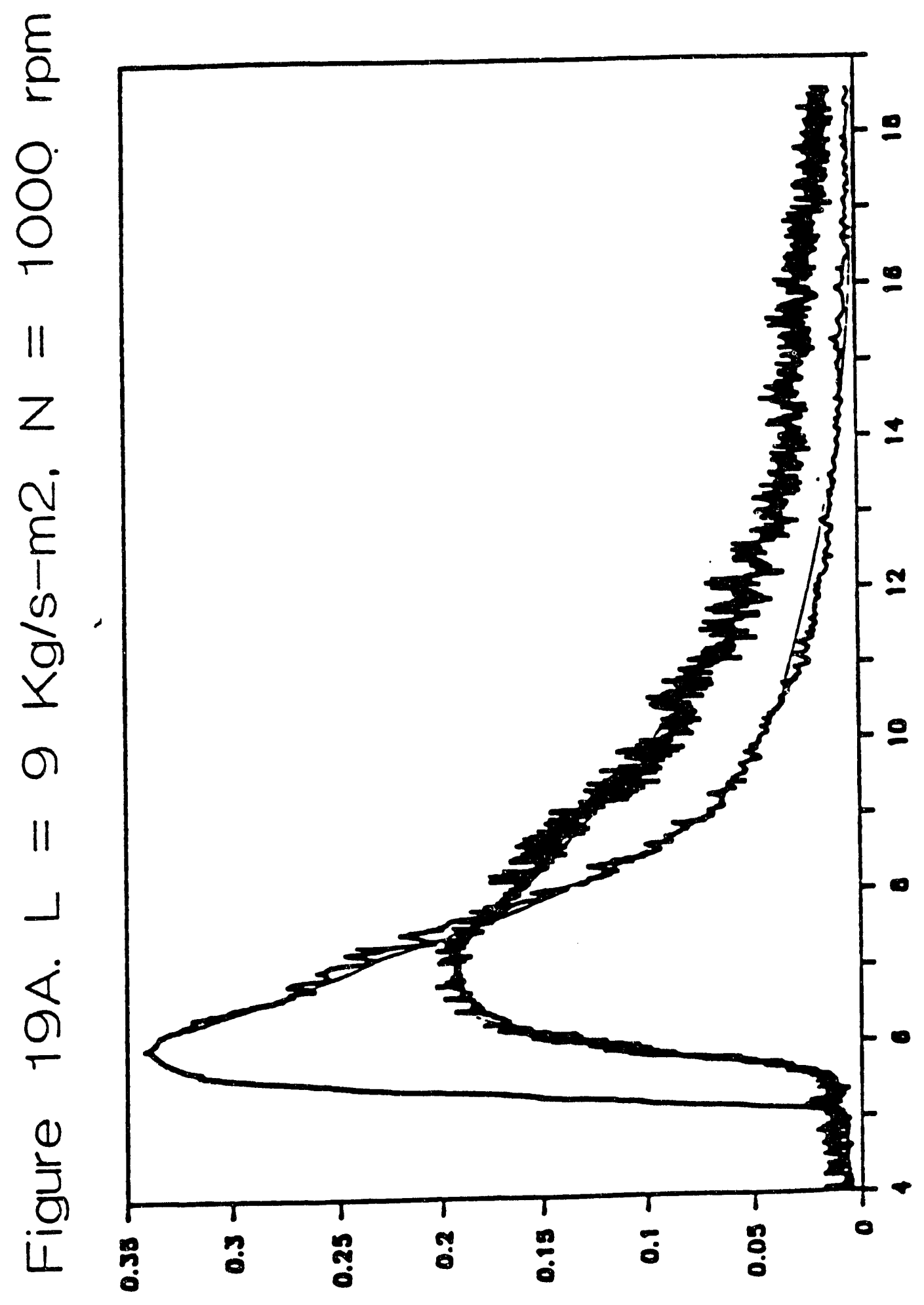




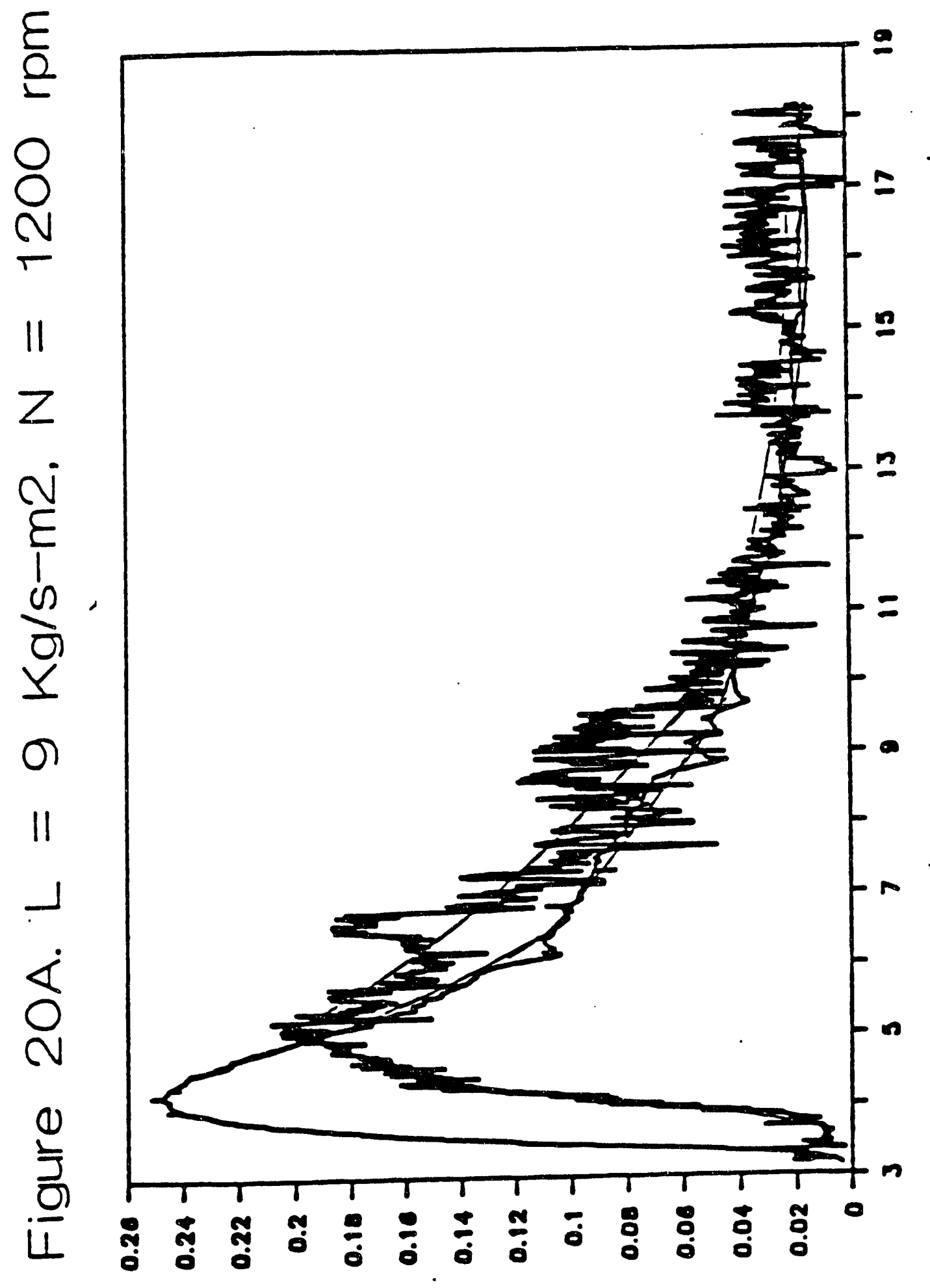




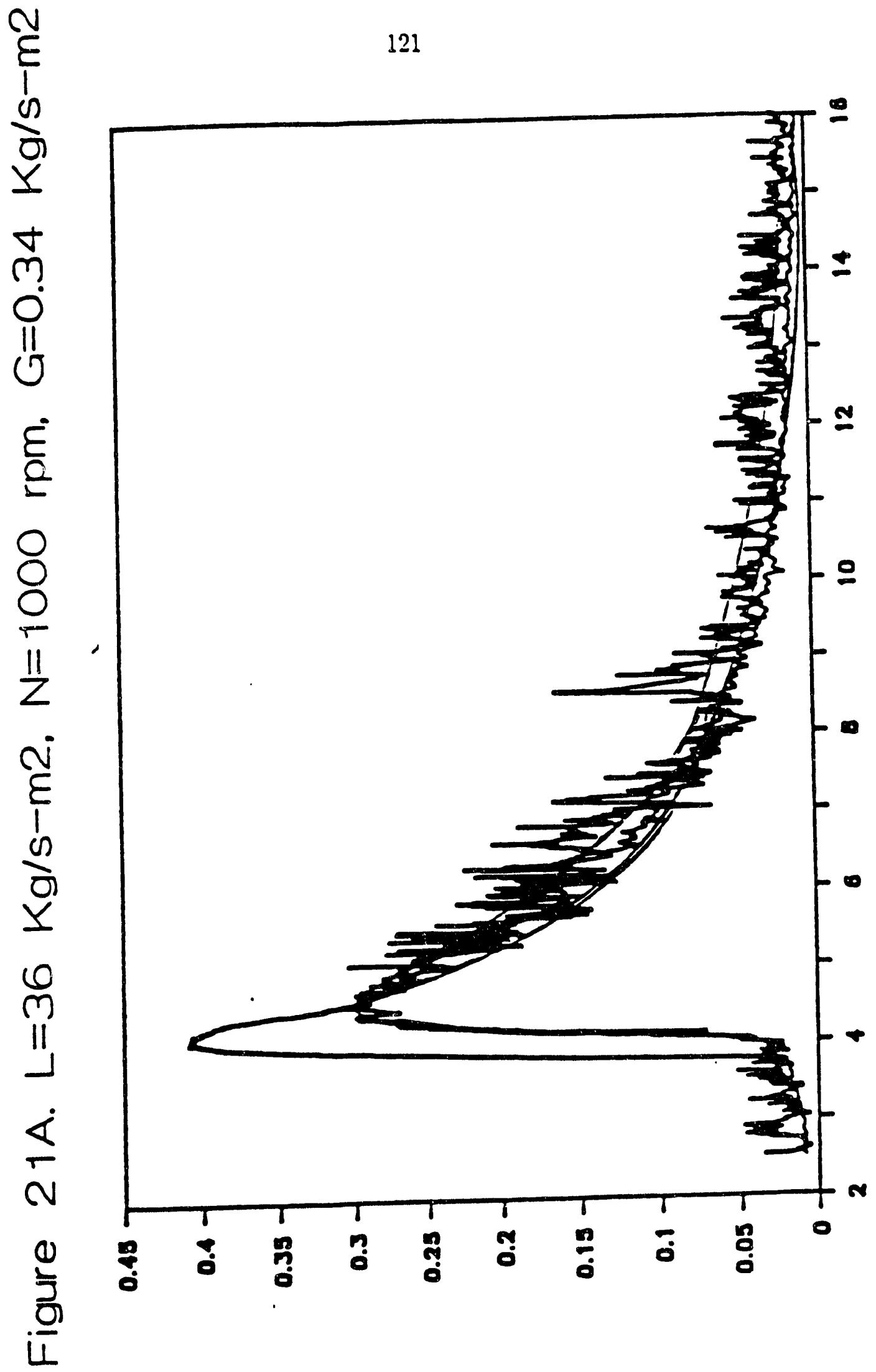




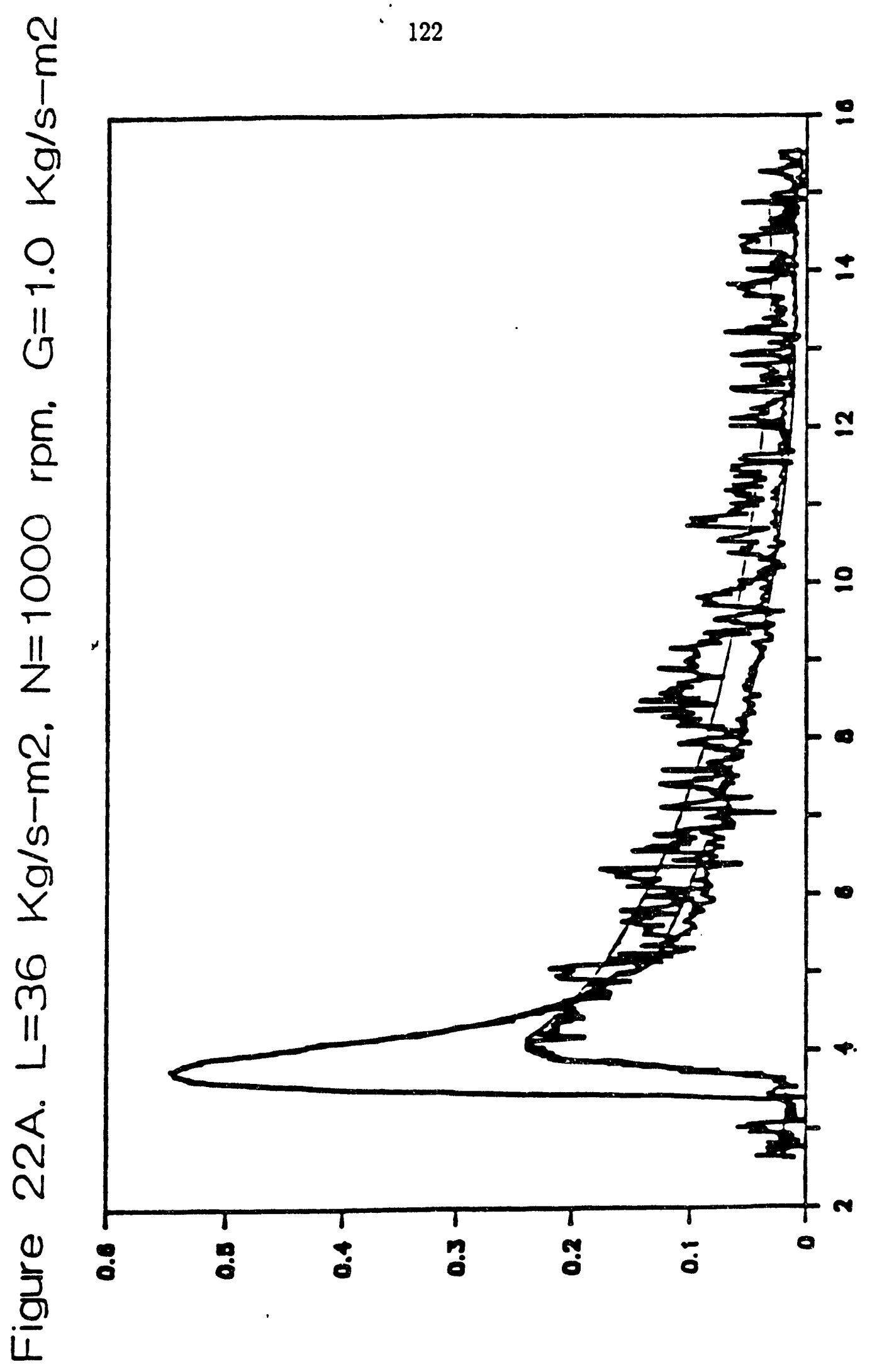




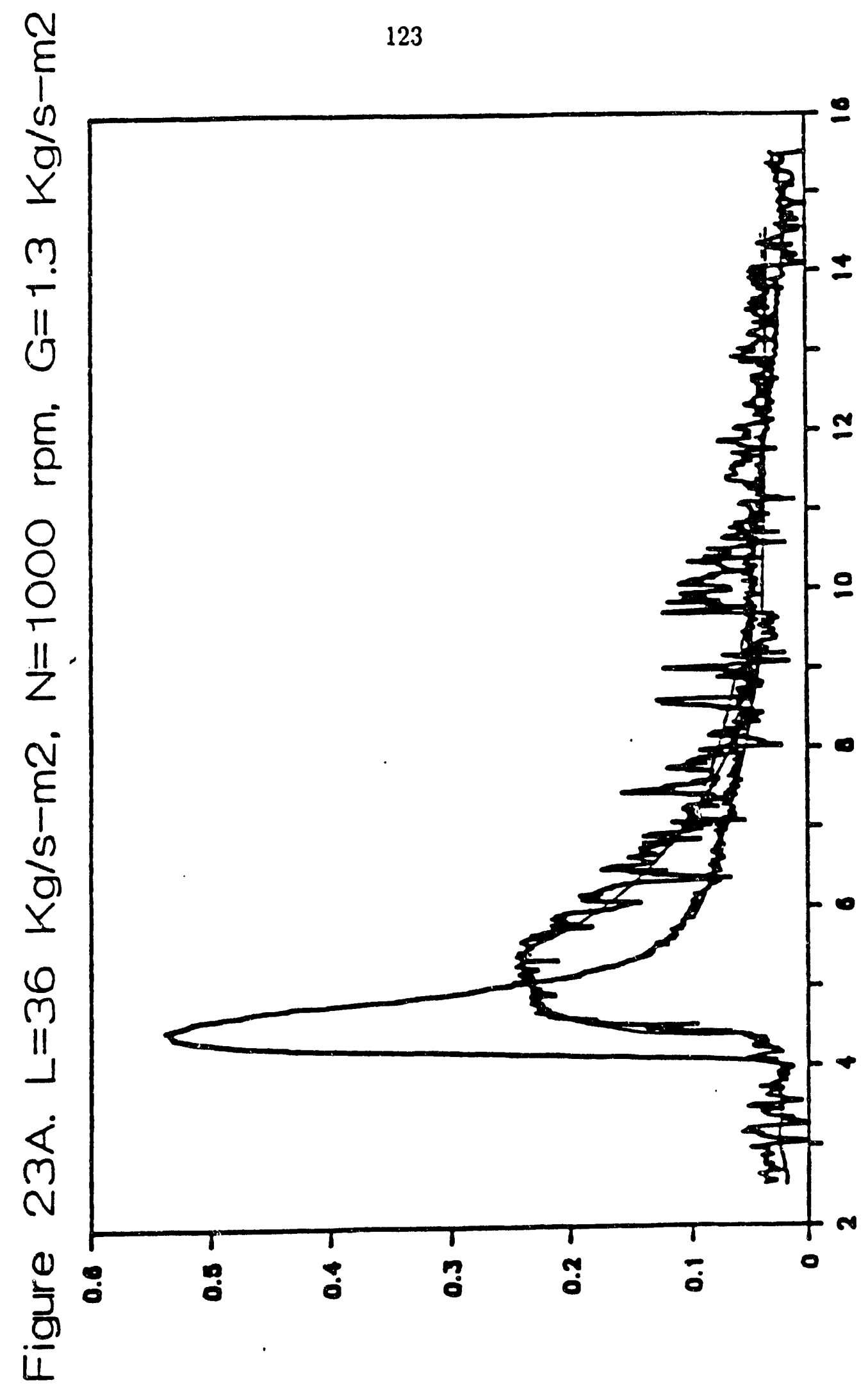




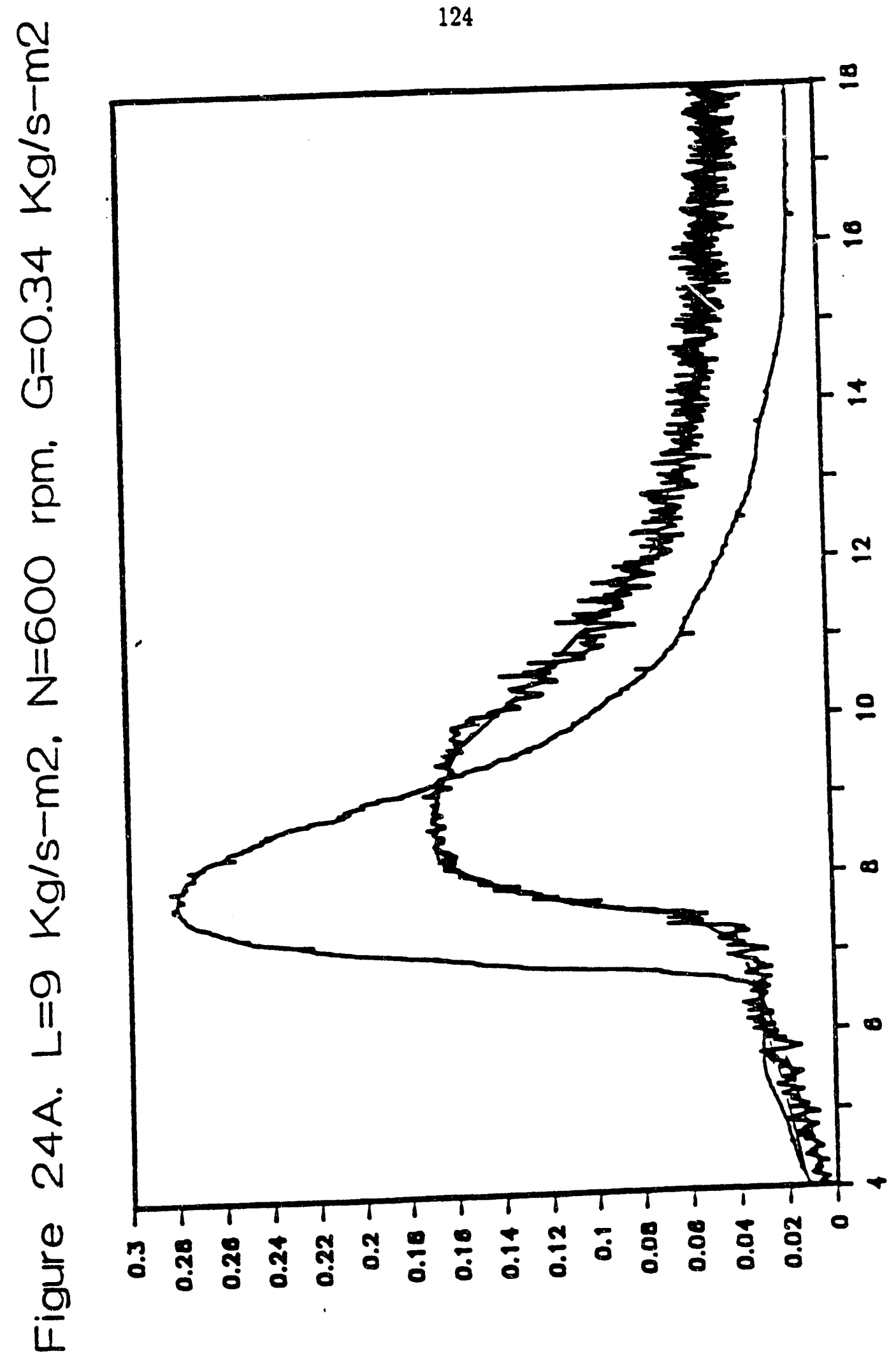




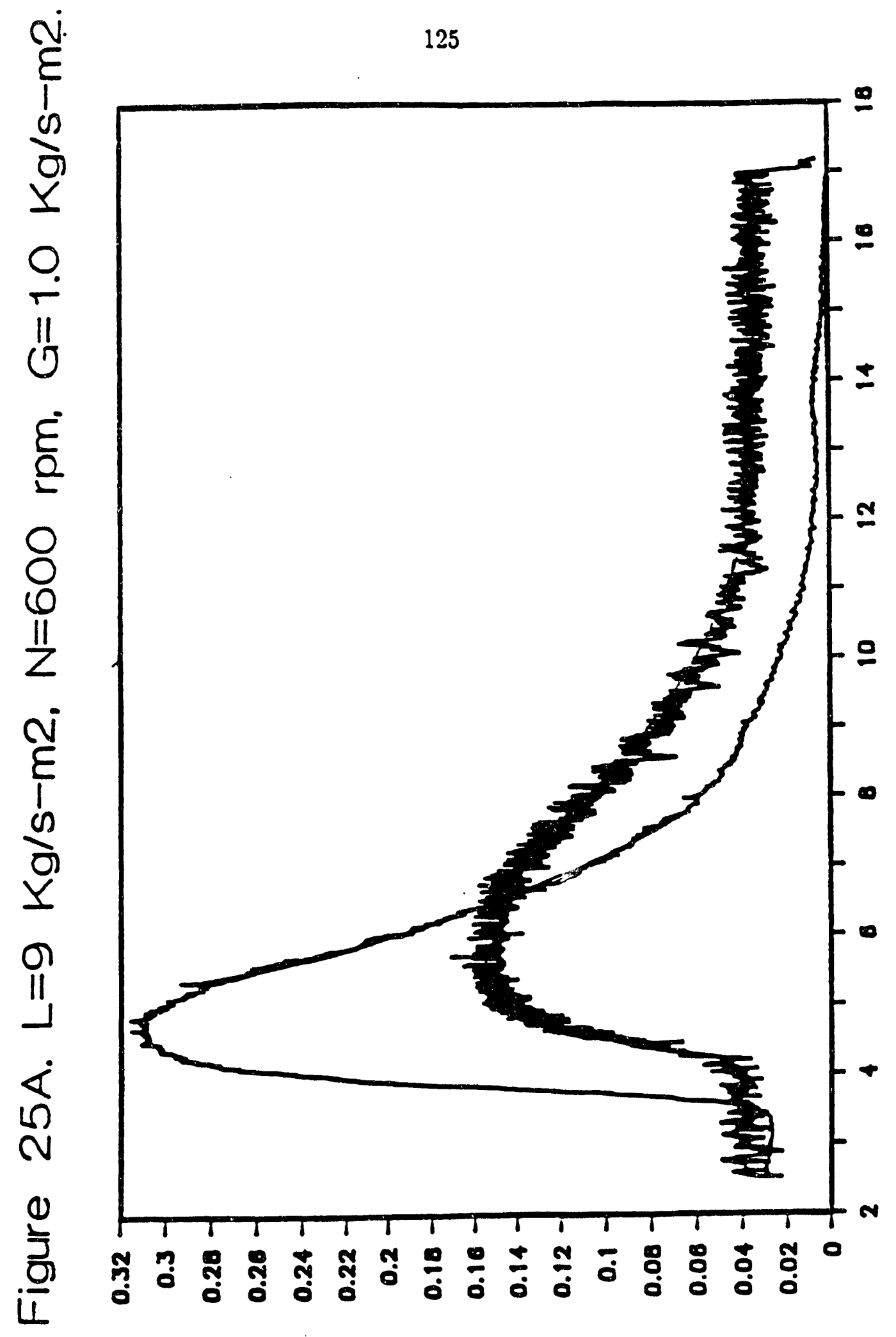




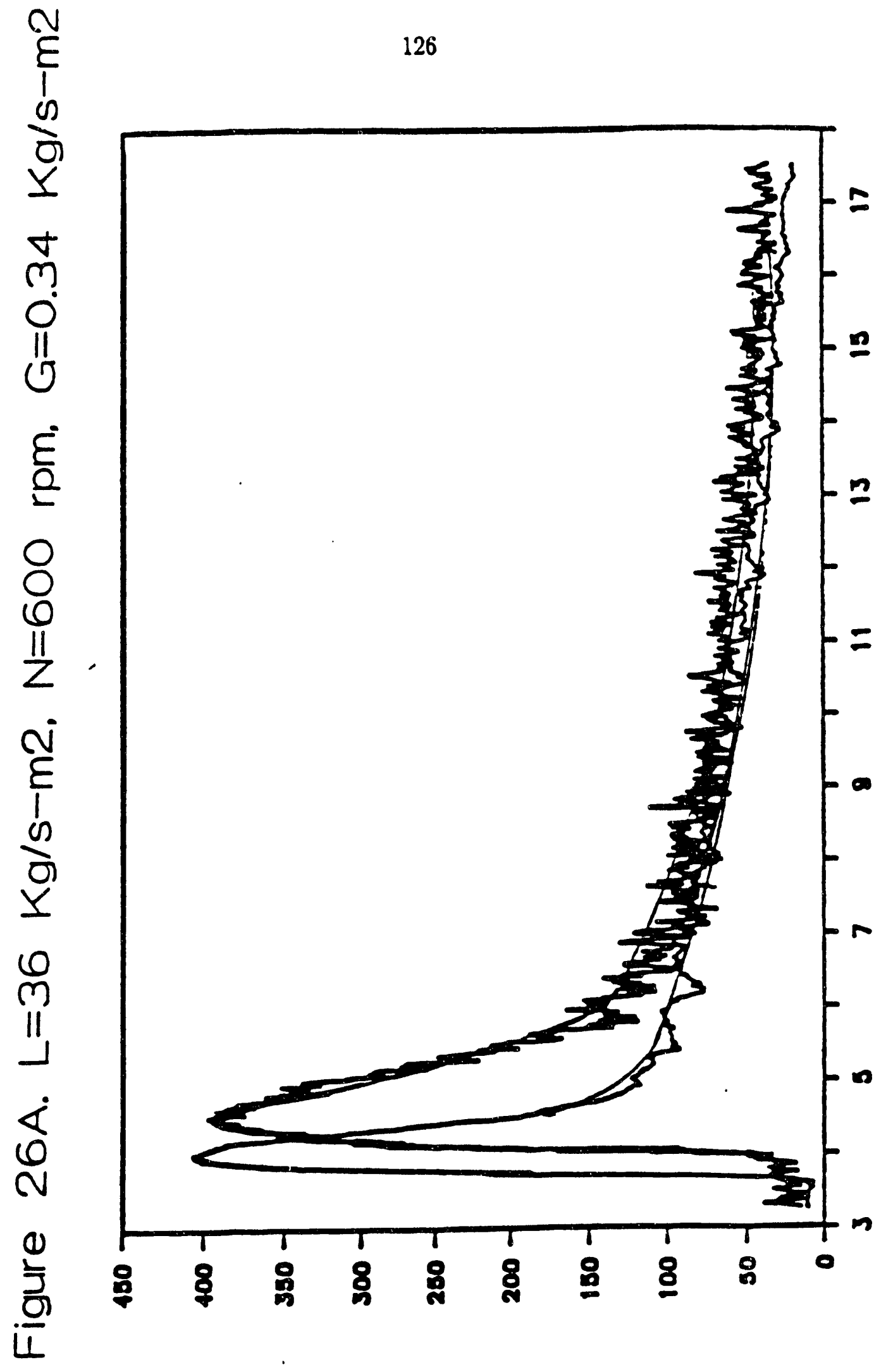




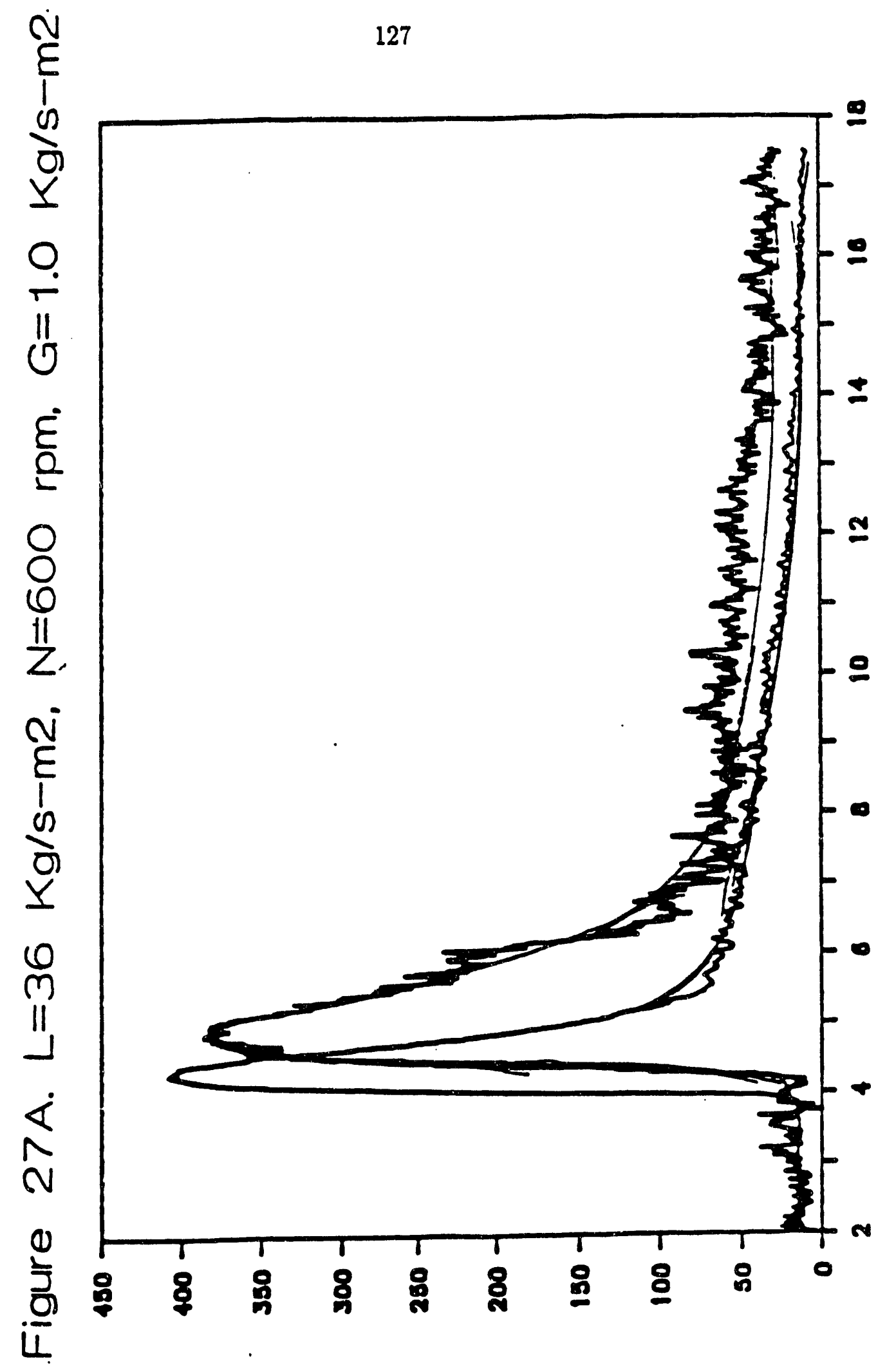




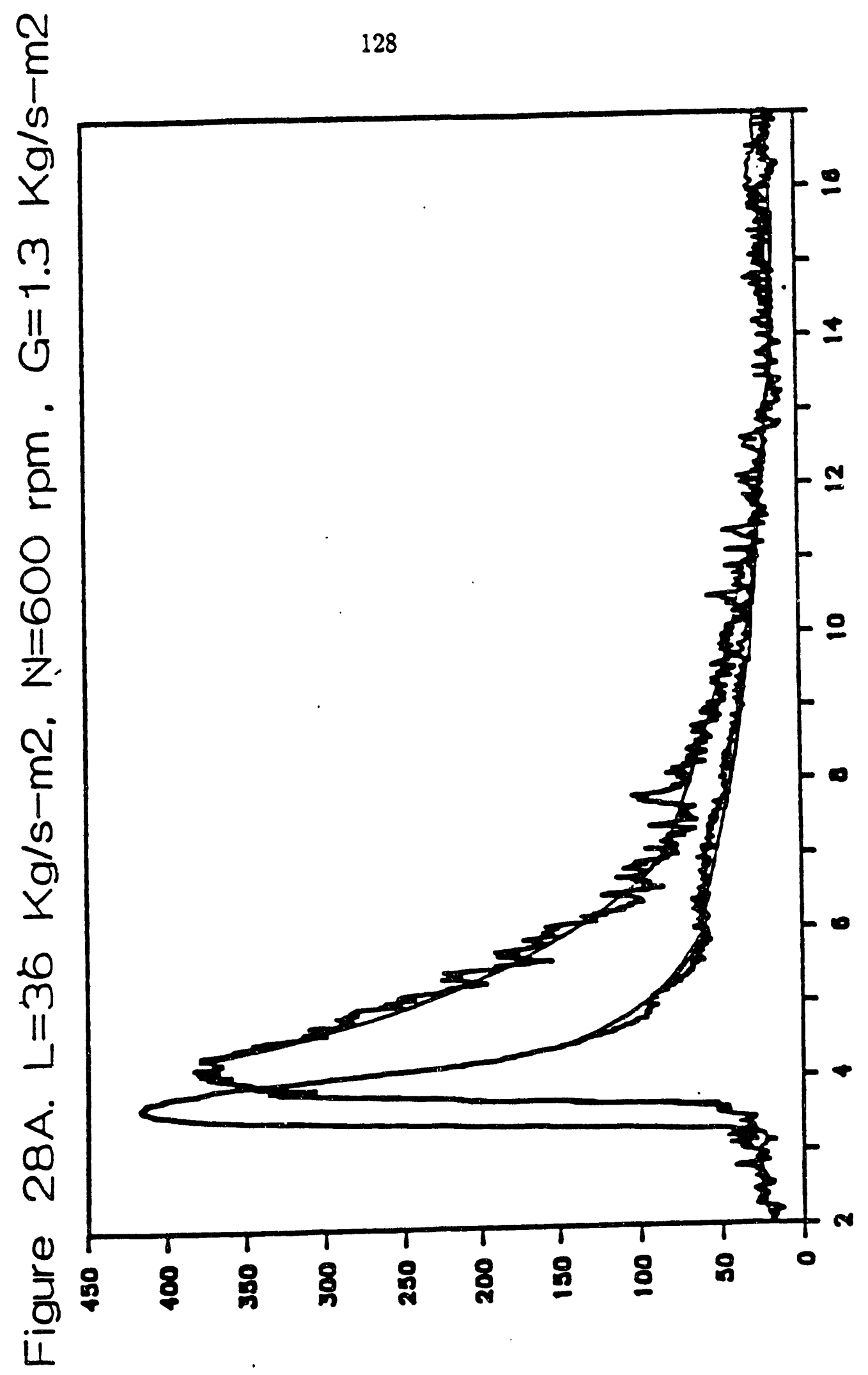


11.6. Selected Coordinate System for the Control Volume:

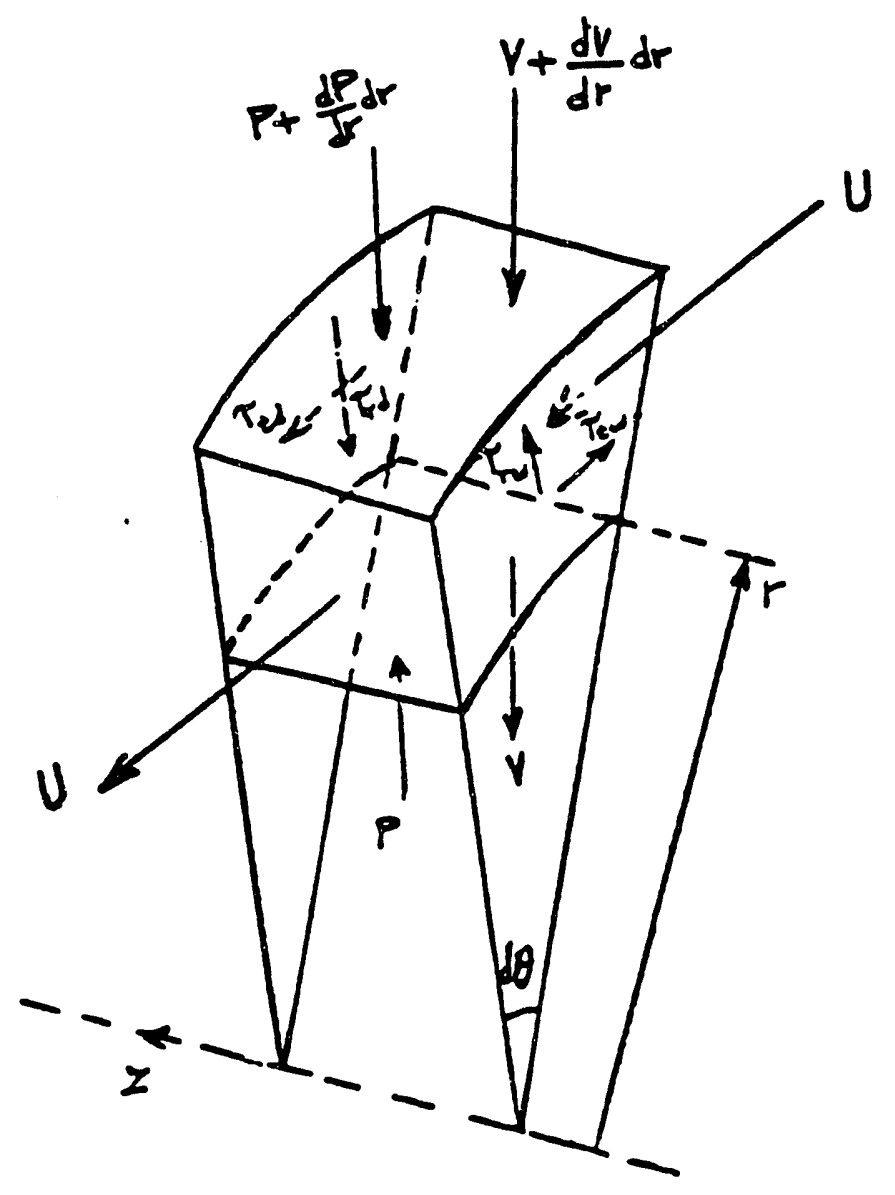




\section{PART II}

\section{ROTATING BED MASS TRANSFER STUDY OF SULFUR DIOXIDE SORPTION IN WATER-LIME SLURRIES}

BY

Adin Coskundeniz 


\begin{abstract}
The effect of gravitational force upon the overall volumetric mass-transfer coefficients in rotating packed bed has been studied for sulfur dioxide absorption in water and in lime slurry. $\mathrm{K}_{\mathrm{C}^{2}}$ is found to increase logarithmically with gravity for both system. The influence of gravity on $K_{\mathbf{C}^{2}}$ increases at lower liquid and higher gas flowrates for $\mathrm{SO}_{2}^{\circ}$-Air-Water system, varying between the power of 0.40 and 0.05 . In $\mathrm{SO}_{2}$-Air-Lime system gravity is found to be less effective as $K_{C^{a}} \propto g^{0.08-0.11}$ and not sensitive to liquid and gas flowrates. For both systems, the overall volumetric mass-transfer coefficients obrained in rotating packed bed are much higher than the corresponding values in conventional packed towers.
\end{abstract}




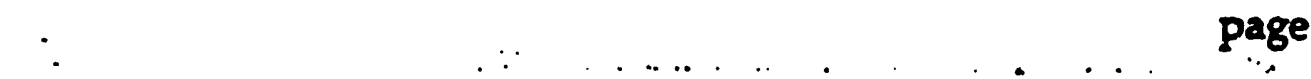

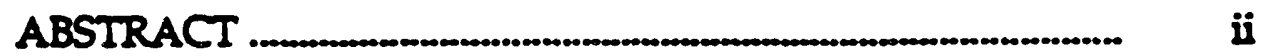

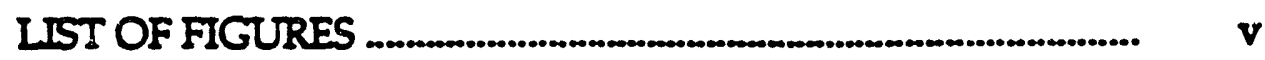

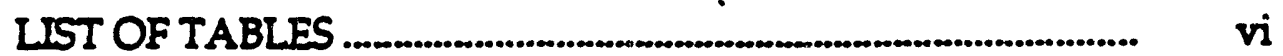

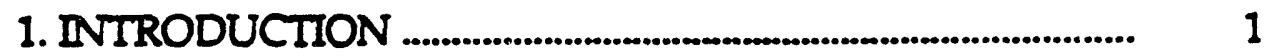

2. PREVIOUS WORK ................................................................. 3

3. EQUIPMENT AND PROCEDURE ............................................ 5

3.1. Schematic of the Rotor ........................................................... 5

3.2. Packing .......................................................................................... 5

3.3. Liquid Distributor ............................................................. 5

3.4. Rotor Assembly and Seals .....................................................

3.5. Flow Diagram ……………........................................................... 7

3.6. Measurements of Operating Parameters .................................. 7

3.7. Data acquisition ......................................................................... 9

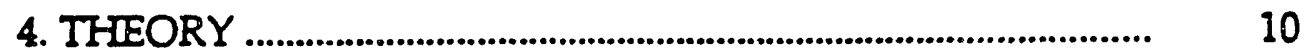

4.1. Two Film Theory ...................................................................... 10

4.2. Calculation of $\mathrm{K}_{\mathrm{L}} \mathrm{a}$ and $\mathrm{K}_{\mathrm{C}} \mathrm{a}$ in Rotating Bed ...................... 16

4.3. Chemistry and Absorption-Rate Steps ................................. 22

4.4. Calculation of $\mathrm{K}_{\mathrm{G}}$ f for $\mathrm{SO}_{2}$-Air-Lime System ...................... 25

4.5. Effect of High Gravitational Field on Absorption

with compared to Conventional Packed Towers .................. 26 


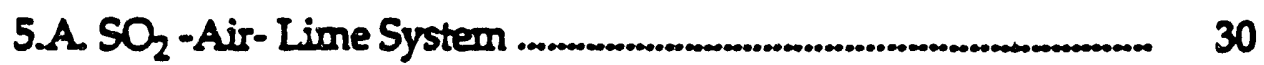

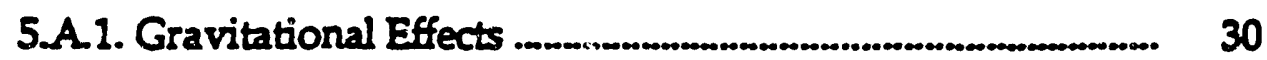

5.A.2 Effect of Liquid Flowrate …......................................... 33

5.A.3. Effect of Gas Flowrate ........................................................... 33

5.A4. Discussion ........................................................................... 33

5.B. $\mathrm{SO}_{2}$-Air-Lime System ......................................................... 37

5.B.1. Effect of Gravity ...................................................................... 37

5.B.2. Effect of Liquidflowrate .......................................................... 37

5.B.3. Effect of Gas Flowrate ............................................................ 42

5.B.4. Discussion …_................................................................. 42

6. CONCLUSION ……................................................................... 46

7. NOMENCLATURE .............................................................. 47

8. REFERENCES …..................................................................... 51

9. APPENDDX …....................................................................... 53 


\section{LIST OF FIGURES}

page

Figure 1. Schematic of Rotating Bed _ 6

Figure 2 Experimental Apparatus ................................... 8

Figure 3. Equilibrium Curve and Operating line

in a packed absorber

Figure 4. Countercurrent Absorption .................................... 17

Figure 5. Equilibrium Species in $\mathrm{SO}_{2}$ Absorption versus pH .. $\quad 23$

Figure 6. Pressure Drop and Flooding Correlations in a packed Tower

Figure 7. $\mathrm{K}_{\mathrm{C}}$ vs $\# \mathrm{~g}$ in $\mathrm{SO}_{2}$-Air-Water System

Figure 8. $\ln K_{c^{a}}$ vs $\ln \# g$

Figure 9. Effect of liquid Flowrate

Figure 10. Effect of Liquid Flowrate

Figure 11. Effect of Gas Flowrate

Figure $12 . \mathrm{K}_{\mathrm{C}^{\mathrm{a}}}$ vs $\# \mathrm{~g}$ in $\mathrm{SO}_{2}$-Air-Lime System

Figure 13. $\mathrm{K}_{c^{a}}$ vs $\#$

Figure 14. In $\mathrm{K}_{\mathrm{C}^{a}}$ vs $\ln \# \mathrm{~g}$

Figure 15. In $\mathrm{K}_{\mathrm{C}^{a}}$ vs $\ln \# \mathrm{~g}$

Figure 16. Effect of Liquid Flowrate

Figure 17. Reproducibility

Figure 18. Effect of Gas Flowrate 


\section{LIST OF TABLES}

Table 1. $\mathrm{SO}_{2}$ absorption in water, $\mathrm{G}=40 \mathrm{acfm}$ 53

Table $2 \mathrm{SO}_{2}$ absorption in water, $\mathrm{G}=20 \mathrm{actm}$. 53

Table 3. $\mathrm{SO}_{2}$ absorption in lime slurry, $\mathrm{G}=40 \mathrm{acfm}$. 54

Table 4. $\mathrm{SO}_{2}$ absorption in lime slurry, $\mathrm{G}=55 \mathrm{adm}$. 54 


\section{INTRODUCTION:}

A radical approach to reduce the capital cost of a chemical plant has been process intensification. Reaction and separation processes are enhanced by the use of high gravitational, electric, acoustic, or magnetic fields. In particular, heat and mass transfer can be intensified ty carrying out processes in high gravitational fields.

High gravitational fields are achieved in rotating packed beds in the form of a rigid metal foam in the shape of a disk with a hole in the center. In gas-liquid mass-transfer operations, liquid is sprayed into the center of the bed and flows outward through the bed because of the centrifugal force. The gas introduced at the outer surface of the packing is forced inward countercurrently to the liquid by the imposed pressure gradient. Intense mass-transfer occurs as high shear forces produce very thin liquid films, rapidly renewed surfaces and substantial turbulance, enhancing the volumetric mass-transfer coefficients. Rotating packed bed has a number of features which differ significantly from more conventional mass transfer devices. Benefits include:

- small size and weight

- reduced solvent inventory

- short residence time

- rapid attainment of steady state

Rotating packed bed has a potantial to be used in flue gas desulfurization (FGD) which is a broadly applied technology for limiting 
sulfur dioxide emissions from power plants, particularly utilizing the high-sulfur coals. Lime slurries is widely used in wet sçubbing methods. This study is primarily concerned with the effect of gravitational force upon the overall volumetric mass-transfer coefficients for sulfur dioxide absorption in water and lime slurry. 


\section{PREVIOUS WORK:}

Vivian et al. [1] presented a correlation for gas absorption in short wetted-wall columns:

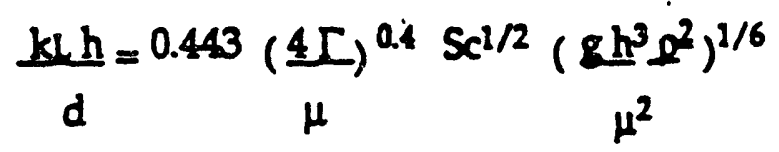

Later, Davidson [2] obtained an exponent of $1 / 6$ on the gravity group by theoretical considerations.

Onda et al. [3] proposed a dimensionless correlations for liquid-phase mass-transfer coefficients in Raschig ring columns. Although the interfacial area, $a$, is assumed to be independent of gravity in previous correlations, $k_{L}$ and $a$ are combined in the expression by Onda et al.

$$
\begin{aligned}
& \int(k L a) d^{2}=0.023 S c^{1 / 2}\left(g d^{3} R^{2}\right)^{0.33}(d L)^{1 / 2} \quad\left(1-1.02 \exp \left[-(0.15)(d L)^{0.4}\right]\right] \\
& \text { D } \\
& \mu^{2} \\
& \mu
\end{aligned}
$$

For the first time, Vivian et al.[4] experimentally studied the effect of gravity on liquid-phase volumetric mass-transfer coefficient for carbon dioxide desorption. In their study, a Raschig-ring cylindirical packed column which is mounted on a rotating centrifuge was used in 1 to 6.4 times the gravity force. Their experimental results showed that $k_{L}$ a varies with gravity to a power between 0.41 and 0.48 , the effect of gravity increasing as the liquid flow rate decreases.

ICI, Imperial Chemical Industries $[5,6]$ introduced the HIIGEE packed bed 
cperated at mean acceleration fields of 200 to $500 \mathrm{~g}$, and reported that volumetric mass transfer coefficient varied with the gravitational force to the 0.14 to 0.54 powers.

Duducovic et al $[7,8,9,10]$ studied the effect of rotational speed on gas-liquid interfacial area and the liquid-side gas-liquid mass-transfer coefficients, separately. They concluded that the volumetric mass-transfer coefficient increases with the gravitational force as a fractional power of 0.30 to 0.37 which is in agreement with the data of Vivian et al. and within the range estimated by ICI. It is shown that experimentally measured gas-liquid interfacial area increases with the gravity as a $\alpha \mathrm{g}^{0.14}$ to $\mathrm{g}^{0.21}$ depending on the packing type and liquid dow rate. The liquid-side gas-liquid mass-transfer coefficient, $k_{L}$ varies with gravity as $k_{L} \propto g^{0.16}$. Power dependency of $1 / 6$ also matches the results obtained from the theoretical predictions based on film flow on rotating disk and rotating blades.

Desorption of carbon dioxide from water into air in rotating packed bed was studied by Keyvani [11]. Results indicates that liquid-phase volumetric mass-transfer coefficient increases with 0.3 to 0.4 power of gravity. 


\section{EQUIPMENT and PROCEDURE:}

\subsection{Schematic of Rotor:}

A simplified schematic of the rotor is shown in Figure 1. The liquid entering from the inner pipe of the stationary double pipe (1) is distributed on the inside edge of the bed (2) and then is accelerated into the housing (3) by the effect of centrifugal force. Due to imposed pressure gradient, the gas entering the housing is flown inward countercurrently to the liquid through the rotating bed. The liquid collects in the bottom of the housing and serves as a gas trap. The gas exits on the inside edge of the bed through the double pipe (4).

\subsection{Packing:}

The rotating packed bed consists of a packing unit, made up of a nickel plated aluminum foam metal in the shape of a torous. The outside diameter is 18 inches and the thickness is 1.75 inches. The packed bed used in the experiments has specific surface area of $900 \mathrm{ft}^{2} / \mathrm{ft}^{3}$ and porosity of 0.92 . The number of pores per inch is $\mathbf{4 0 . 6}$ and pore size is 0.01 . Packing unit is dimensionally stable in the high gravitational fields.

\subsection{Liquid Distributor.}

The liquid distributor has been designed to eliminate the liquid entrainment problems and to provide even liquid distribution at the inner edge of the rotating bed where entrainment of liquid by gas is most probable to occur due to high gas and low liquid flow rates. Distributor consists of a 
6

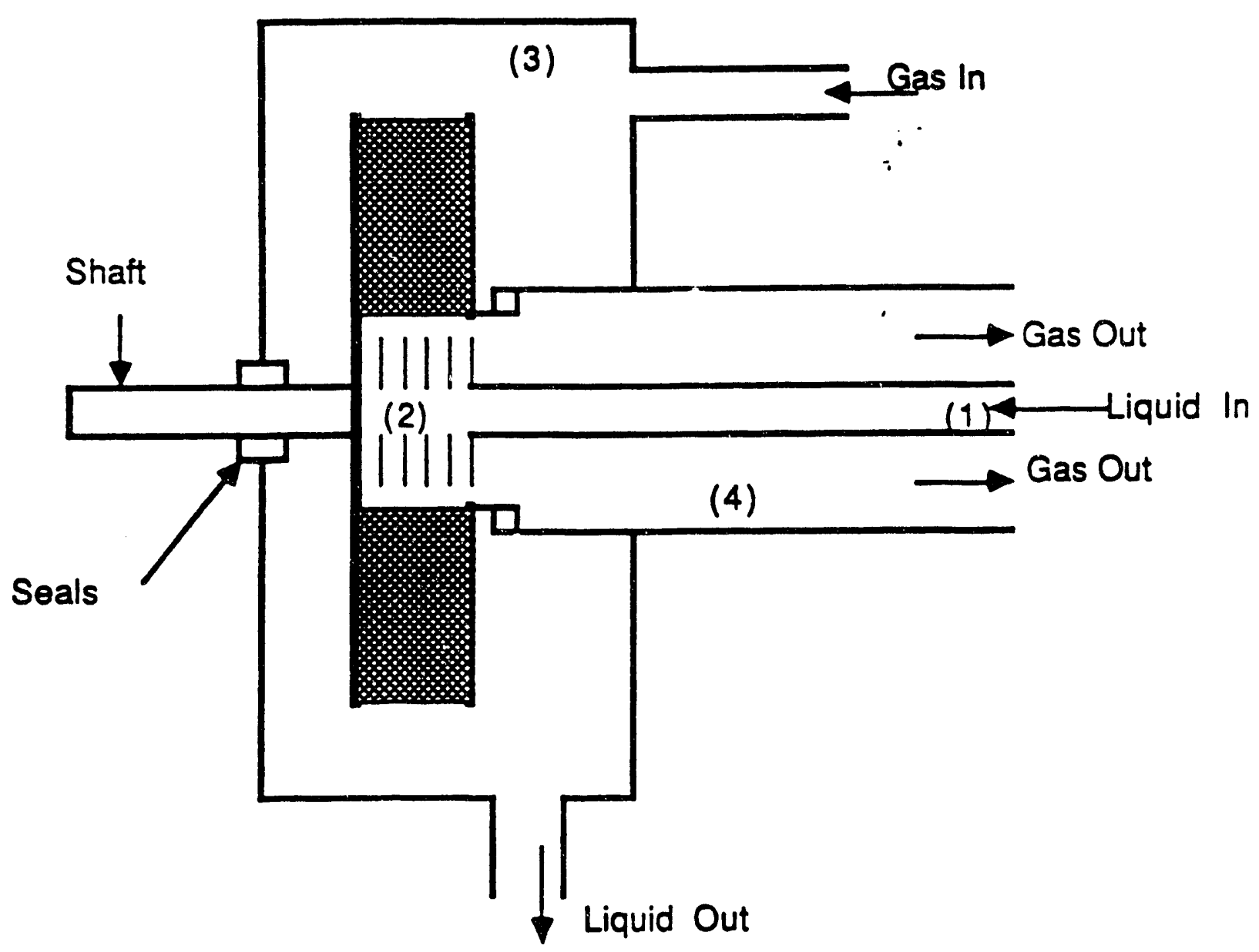

Figure 1. Schematic of Rotating Bed 
stainless steel pipe (3/4 " $\mathrm{D}, 3-1 / 2$ " " long) with a flattened end. About 1/16 "-thick liquid sheet is produced by using flattened end which forms a 1-1/2 " by $1 / 16$ " slit. The liquid distributor is placed $1 / 2$ " above the inner edge of the rotating bed.

\subsection{Rotor Assembly and Seals:}

The rotor assembly is holded by a 2 inches diameter-shaft which is supported by two bearings. The assembly is driven by a 5 hp-motor ( 3 phase, $230 \mathrm{~V}$ ) with a belt and a speed controller (Parajust Model M01712-A00).

Silicon rubber radial lip seals are placed in the shaft and the double pipe-gas exit assemly.

\subsection{Flow Diagram:}

Flow diagram of the experimental apparatus which have been uset in the absorption of $\mathrm{SO}_{2}$ both in water and lime slurry, is shown in Figure 2 The maximum liquid flow rate of $30 \mathrm{gpm}$ can be achieved by using the feed and recycle pumps. Up to $60 \mathrm{acfin}$ of air can be delivered to the system by the compressor used. A static mixer is placed in the piping system in order to provide a well-mixed $\mathrm{SO}_{2}$-Air mixture before gas inlet.

\subsection{Measurement of Operating Parameters:}

The gas flow rates were measured by correlating the pressure drop across restriction orifices which was determined by using differential pressure transducers. Pure $\mathrm{SO}_{2}$ flow rate is adjusted by a rotameter.

The liquid flow rate is determined by Magnetic Flowmeter (Magnavax 


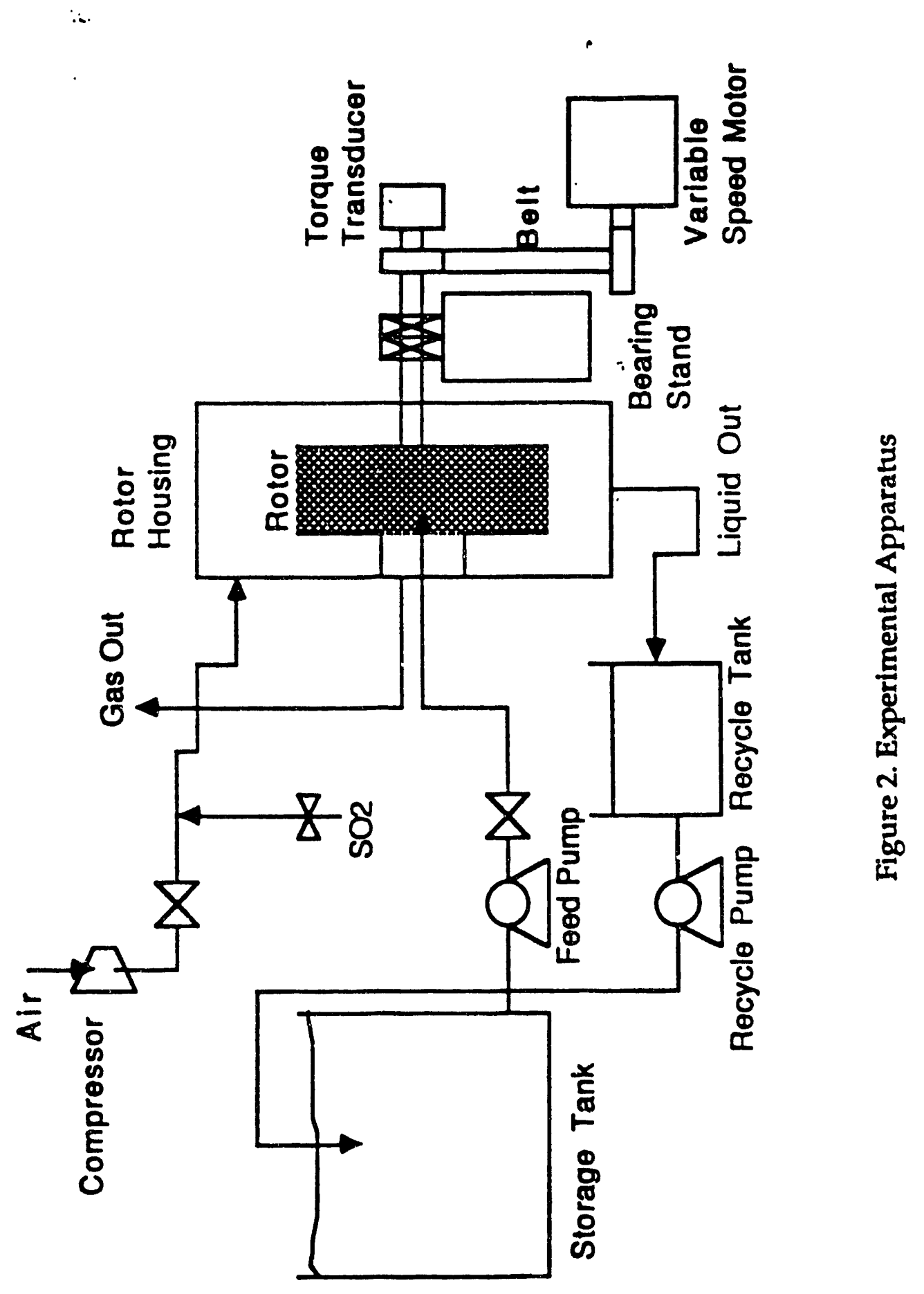


Model Mag 3570) which is capable of measuring flowrates up to $10 \mathrm{gpm}$. Orifice has not been used for measuring lime-slurry flow because of its highly abrasive nature.

A pressure gage was used to monitor the pressure of the liquid entering the rotating bed.

In order to measure pressure drop across the bed, probes for the transducers were placed at the inlet and outlet of the rotor as shown in Figure 1.

Thermocouples are placed in the inlet and outlet of the rotor to monitor the temperature across the bed which has great influence on the solubilty data.

The torque required for the rotating bed was measured by a sprocket/pulley torque transducer (Lebow Model 1388-102) which is connected to the end of the drive shaft. The speed of rotation was measured by a magnetic pick-up tachometer (Amtex Model 1736).

Sulfur dioxide concentrations in the gas mixture were determined by infrared $\mathrm{SO}_{2}$ analizer (Horiba Model VIA-300) which is continuously connected to the inlet and outlet of the rotor.

\subsection{Data Acquisition:}

The gas and liquid flow rates, pressure drop across the bed, rotational speed and torque are interfaced to a personel computer (IBM-XI). Labtech Notebook, a menu driven software package is used for data acquisition in actual units. 


\section{THEORY:}

\subsection{Two Füm Theory:}

The two film theory, originally proposed by Whitinan [12] is widely used to explain mass transfer operations, such as absorption. In this theory, the interface between the gas phase and the liquid phase is assumed to consist of a gas film adjacrnt to a liquid film. It is presumed that the main body of gas and liquid phase are completely mixed by turbulent flow, while the gas and liquid film are laminar or stagnant. There is no solute concentration gradient in the main body of either phase, but a solute concentration gradient exsist across both the gas film and the liquid film. At the interface, solute concentration in the gas film is assumed to be in equilibrium with the solute concentration in the liquid film. There is no resistance to mass transfer at the interface. In the absorption operations where solute is transferred from a vapor phase to a liquid phase, the solute concentration in the vapor phase should be greater than the equilibrium concentration in the liquid phase in order to drive the mass transfer. A solute in the main body of gas phase should first pass through the gas film, then through the liquid film, and finally dissolve into the main body of liquid.

The solute transferred per unit time from the main body of gas phase through the gas film is:

$$
N=A Z k_{C} a\left(p-p^{\prime}\right)
$$

Partial pressure of solute in the gas phase is related to mole fraction as follows:

$$
p=y P_{T}
$$


Equation (1) and (2) may be combined as:

$$
N=A Z k_{C} a P_{T}\left(y-y^{\prime}\right)
$$

Similarly, the solute transferred per unit time through the liquid film to the main body of liquid phase is given by equation (4):

$$
N=A Z k_{L} a\left(x^{\prime}-x\right)
$$

where $\mathrm{N}=$ solute transferred $(\mathrm{lb}-\mathrm{mol} / \mathrm{h})$

$A=$ column cross-sectional area $\left(f t^{2}\right)$

$Z=$ height of packed tower $(f t)$

$\mathrm{kC}=$ gas-phase mass-transfer coefficient ( $\left.\mathrm{b}-\mathrm{mol} / \mathrm{h} \cdot \mathrm{ft}^{2} \cdot \mathrm{atm}\right)$

$k_{L}=$ liquid-phase mass-transfer coefficient $\left(\mathrm{b}-\mathrm{mol} / \mathrm{h} \cdot \mathrm{ft}^{2} \cdot \mathrm{m} \cdot \mathrm{ol} / \mathrm{mol}\right)$

$a=$ interfacial area $\left(\mathrm{ft}^{2} / \mathrm{ft}^{3}\right)$

$P_{\mathrm{T}}=$ total system pressure (atm)

$\mathrm{y}=\mathrm{mol}$ fraction of solute in gas phase

$y^{\prime}=$ mol fraction of solute in the gas phase at interface

$x=$ mol fraction of solute in liquid phase

$x^{\prime}=$ mol fraction of solute in the liquid phase at interface

The interfacial area is considered to be the actual mass transfer area between liquid and gas phases. The product of $\mathrm{a}$ and $\mathrm{A} \mathrm{Z}$ gives the total interfacial area within the entire packed bed.

Since concentration differences, such as $\left(y-y^{\prime}\right)$, take place over extremely small distances, it is difficult to determine the values of $p^{\prime}$ and $x^{\prime}$ in practical cases. However, it is assumed that the concentrations in the gas and liquid 
phases are corelated by a linear equilibrium relationship over a small solute consentration change. In this case, the rate of mass transfer is proportional to the differences between the main body concentration in one phase and the concentration which would be equilibrium with the main body concentration in the second phase. Therefore, the solute transferred can be written in terms of overall mass-transfer coefficients $K_{C}$ and $K_{L}$ shown in equation (5) and (6).

$$
\begin{aligned}
& N=A Z K_{C^{a}} P_{T}\left(y-y^{*}\right) \\
& N=A Z K_{L} a\left(x^{*}-x\right)
\end{aligned}
$$

Here, $y^{*}$ is the solute mole fraction in the gas phase which would be in equilibrium with the liquid phase and similarly, $x^{*}$ is the the solute mole fraction in liquid phase which would be in equilibrium with the gas phase. In these equations, overall gas and liquid-phase mass transfer coefficients, $K_{C}$ and $K_{L}$, are combined with the interfacial area, $a$, to produce overall gas and liquid-phase volumetric mass-transfer coefficients, $\mathrm{K}_{\mathrm{C}^{a}}$ and $\mathrm{K}_{\mathrm{L}} \mathrm{a}$. Equilibrium curve and operating line in a packed absorber is shown in Figure 3. The equilibrium curve slope, $m$, is defined from Figure 3.

$$
m_{1}=\frac{y-y^{\prime}}{x^{*}-x^{\prime}} \quad \text { (n) or } m_{2}=\frac{y^{\prime}-y^{\prime \prime}}{x^{\prime}-x}
$$

Equation (3) can be rewritten as:

$$
y-y^{\prime}=\frac{N}{A Z k_{C} P_{T}}
$$




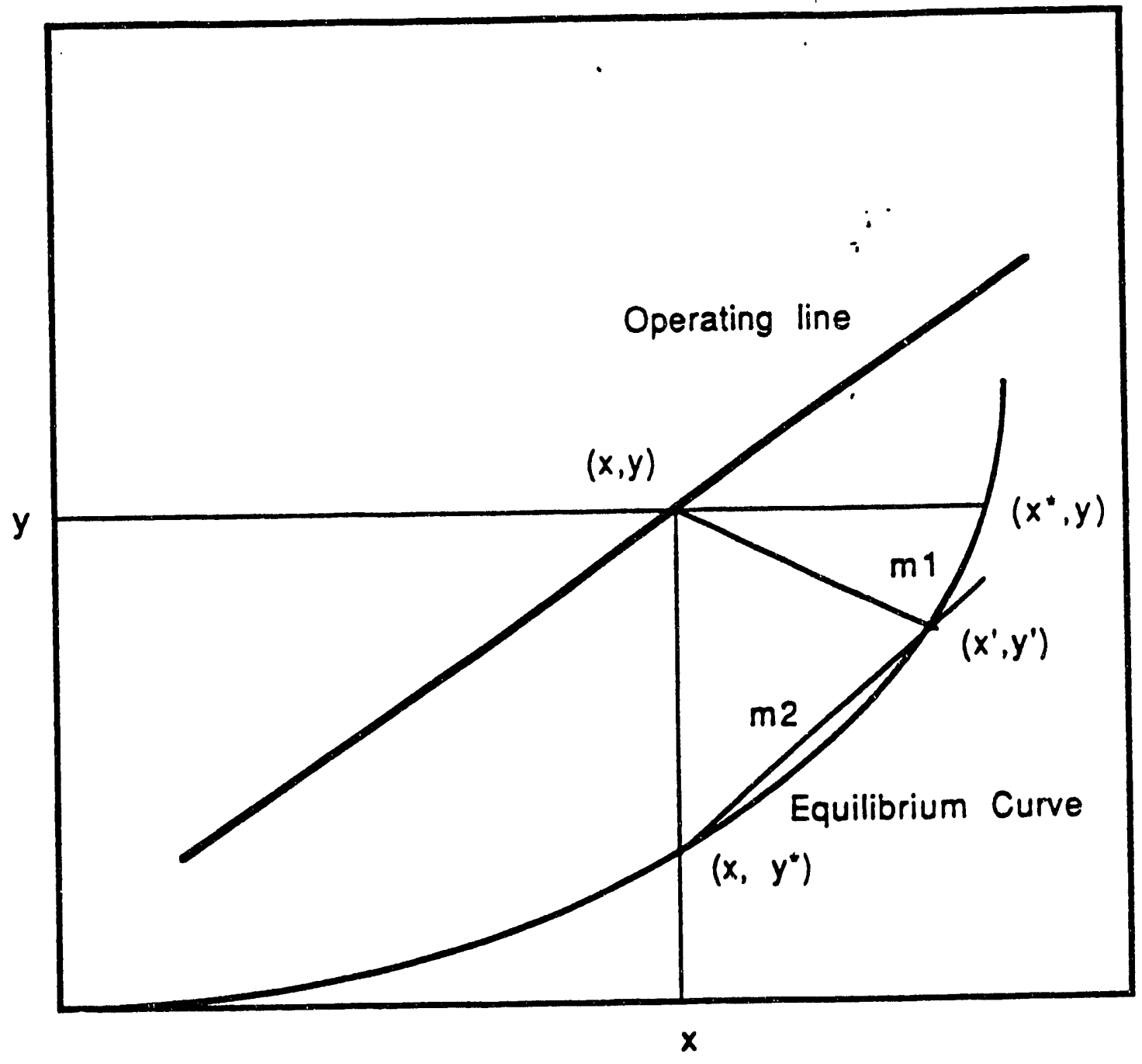

Figure 3. Equilibrium Curve and Operating Line in an Absorber 
Equation (8) is substituted into equation (4), so that

$$
y^{\prime}-y^{+}=\frac{m^{2} N}{A Z k_{L} a}
$$

and adding the driving forces yields: .

$$
y-y^{*}=y-y^{\prime}+y^{\prime}-y^{*}=\frac{N}{A Z k_{C} a P_{T}}+\frac{m_{2} N}{A Z k_{L} a^{\prime}}
$$

The interfacial concentration is eliminated, giving equation (12).

$$
y-y^{*}=\frac{N}{A Z}\left(\frac{1}{k_{G^{a}} P_{T}}+\frac{m z}{k_{L} a}\right)
$$

The substitution of equation (5) into equation (12) gives:

$$
\frac{1}{K_{C} a P_{T}}=\frac{1}{k_{C} a P_{T}}+\frac{m 2}{k_{L} a}
$$

Here, $\mathrm{K}_{\mathrm{C}} \mathrm{a}$ is overall gas-phase volumetric mass-transfer coefficient expressed in the units of $\left(\mathrm{lb}-\mathrm{mol} / \mathrm{h} \cdot \mathrm{ft}^{3} \cdot \mathrm{atm}\right)$. This equation shows how the overall gas-phase mass-transfer coefficient is related to the individual gas and liquid-phase coefficients. The reciprocals of mass-transfer coefficients in the above equation, are regarded as the resistances to mass transfer, consisting of a gas-phase resistance and a liquid-phase resistance in series.

Similarly, the overall resistance to mass transfer in terms of liquid phase can be found as follows:

$$
x^{*}-x=x^{*}-x^{\prime}+x^{\prime}-x
$$


Equation (4) and (7) can be substituted into equation (14).

$$
x^{*}-x=\frac{N}{m_{1} A Z k_{\sigma^{2}} P_{T}}+\frac{N}{A Z k_{L^{2}}}
$$

When equation (6) is taken into account, it becomes

$$
\frac{1}{K_{L} a}=\frac{i}{m_{1} k_{C^{a}} P_{T}}+\frac{1}{k_{L} a}
$$

Here, $\mathrm{K}_{\mathrm{L}} \mathrm{a}$ is the overall liquid-phase volumetric mass-transfer coefficient expressed in the units of $\left(\mathrm{lb}-\mathrm{mol} / \mathrm{h} \cdot \mathrm{ft}^{3} \cdot \mathrm{mol} / \mathrm{mol}\right)$.

When equilibrium line is linear in which the case of $\left(m=m_{1}=m_{2}\right)$ and $k_{G}$ is small compared to $k_{L}$ or $m$ is small (equilibrium line flat), solute is very soluble in the liquid. In equation (13), $m / k_{L} a$ term becomes minor and $1 / k_{C^{a}}$ term represents the major resistance. The absorption is considered to be gas-phase mass-transfer controlled in which $K_{C^{a}}{ }=k_{C}{ }^{a}$

Conversely, when $k_{L}$ is small compared to $k_{G}$ or $m$ is very large (equilibrium line vertical), solute is insoluble in the liquid. In equation (16), $1 / m k_{C} a P_{T}$ term can be neglected and the major resistance $\left(1 / k_{L} a\right)$ is considered to exist in the liquid- phase $\left(K_{L} a-k_{L} a\right)$. The absorption, having these characteristics are called liquid-phase controlled.

$K_{\sigma^{a}}$ is most useful in designs when composition of the gas phase is of primary interest and $\mathrm{K}_{\mathrm{L}}$ where composition of the liquid phase is of primary interest. 


\section{Calculation of $K_{L^{2}}$ and $K_{G^{a}}$ in Rotating Bed:}

Generally, height of a transfer unit is used to present data on absorption column performance Because of simplicity and similarity to plate-column calculation methods, design procedures are based on this concept. The total height of a packed bed is given by:

where $\begin{aligned} Z & =(\text { HTU }) \times(N T U) \\ Z & =\text { depth of packing }(\mathrm{ft}) \\ \text { HTU } & =\text { height of a transer unit }(\mathrm{ft}) \\ \mathrm{NTU} & =\text { number of transfer units }\end{aligned}$

Figure 4 shows the rotating packed bed together with the nomenclature that will be used in developing the equations.

When operating and equilibrium lines are linear over the concentration range of the column, it is practical to employ the use of a logarithmic mean driving force in the rate equations in which the overall volumetric mass-transfer coefficients are used.

The rate of interphase transfer can be expressed in terms of $\mathrm{K}_{\mathrm{L}} \mathrm{a}$ :

$$
N=G\left(y_{i}-y_{0}\right)=L\left(x_{0}-x_{i}\right)=K_{L} \text { aA } Z p\left(x^{*}-x\right)_{L M}
$$

where

$$
\left(x^{4}-x\right)_{L M}=\frac{\left(x^{4}-x\right)_{0}-\left(x^{4}-x\right)_{i}}{\ln \frac{\left(x^{4}-x\right)_{0}}{\left(x^{4}-x\right)_{i}}}
$$

Assuming that gas and liquid flowrates are constant and Henry's law is obeyed for the dilute system, 


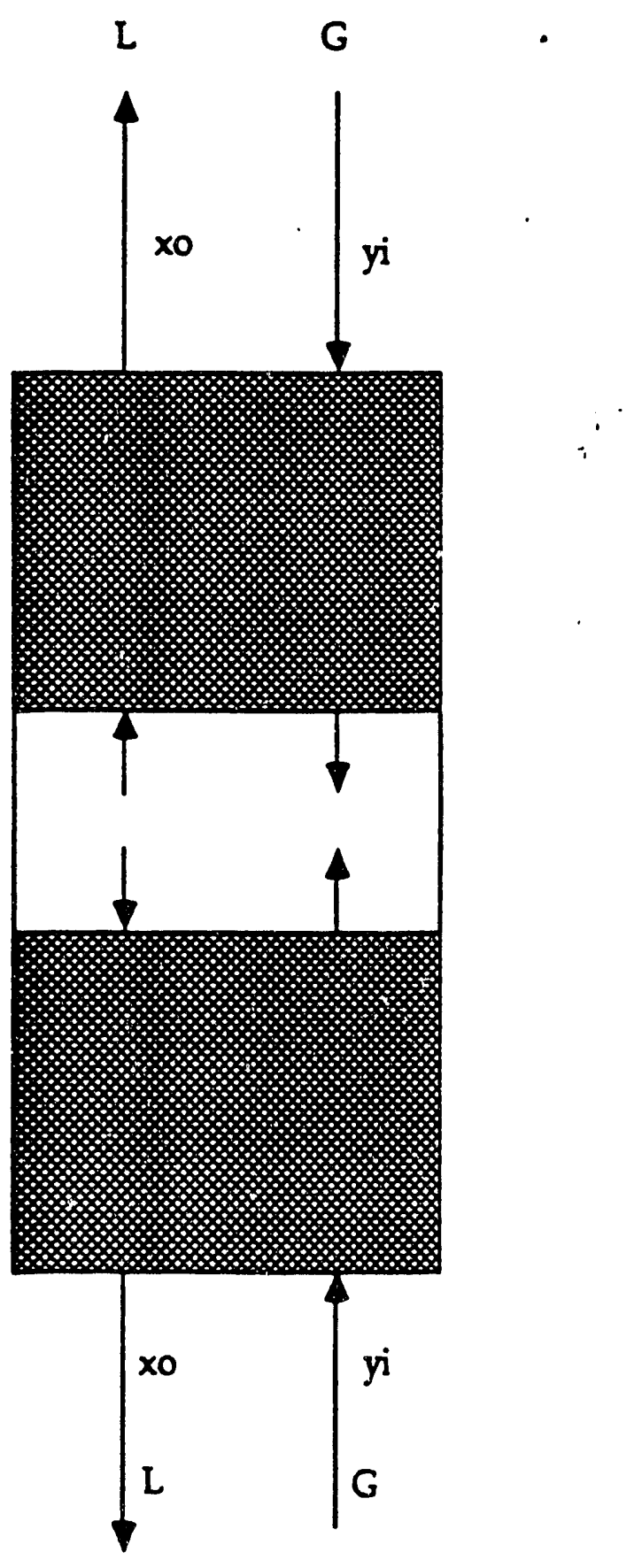

Figure 4. Countercurrent Absorption in Rotating Packed Bed 


$$
\mathrm{P}=\mathrm{HC} \quad \text { (20) }
$$

Here, $\mathrm{p}$ is solute partial pressure in gas phase (atm), $\mathrm{c}$ is solute concentration in liquid (lb-mol/ $\left.\mathrm{ft}^{3}\right), \mathrm{H}$ is Henry's law constant (atm/ $\left(\mathrm{bb}-\mathrm{mol} / \mathrm{ft}^{3}\right)$ ), and $\mathrm{Hr}$ is also Henry's law constant which is the reciprocal of $\mathrm{H}$.

Henry's law constant can be correlated to the slope of equilibrium line as:

$$
\mathrm{m}=\frac{\mathrm{H}_{\mathrm{O}}}{\mathrm{P}_{\mathrm{T}}} \quad(21)
$$

The ligutid phase solute concentration which is in equilibrium with gas phase in terms of $m$ is:

$$
x^{*}=y / m \quad(22)
$$

The depth of packing can be estimated by equation (23).

$$
Z=\frac{L}{K_{L} a A p} \frac{\left(x_{0}-x_{i}\right)}{\left(x^{4}-x\right)_{L M}}
$$

where

$$
\begin{aligned}
& H T U=\frac{L}{K_{L} a A \rho}=\frac{L}{K_{L} a \rho} \\
& N T U=\frac{\left(x_{0}-x_{i}\right)}{\left(x^{4}-x\right)_{0}-\left(x^{4}-x\right)_{i}} \ln \frac{\left(x^{4}-x\right)_{0}}{\left(x^{4}-x\right)_{i}}
\end{aligned}
$$

In the above equations, $\mathrm{N}$ is $\mathrm{SO}_{2}$ transferred from gas phase to liquid phase 
$(\mathrm{bb}-\mathrm{mol} / \mathrm{h}), \mathrm{G}$ is the molar gas flow rate $(\mathrm{bb}-\mathrm{mol} / \mathrm{h}), \mathrm{L}$ is molar liquid flow rate $(\mathrm{lb}-\mathrm{mol} / \mathrm{h}), \mathrm{L}$ ' is the average of the molar liquid flow rates per unit area based on the inner and outer cross-sectional area of rotating bed $\left(\mathrm{lb}-\mathrm{mnol} / \mathrm{ft}^{2} \cdot h\right), \mathrm{P}_{\mathrm{T}}$ is total system pressure (atm), $\mathrm{K}_{\mathrm{L}}$ is the overall liquid-phase mass-transfer coefficient $\left(\mathrm{lb}-\mathrm{mol} / \mathrm{h} \cdot \mathrm{ft}^{2} \cdot\left(\mathrm{lb}-\mathrm{mol} / \mathrm{ft}^{3}\right)\right)$ or $(\mathrm{ft} / \mathrm{h}), \mathrm{A}$ is packing cross-sectional area $\left(\mathrm{ft}^{2}\right), p$ is the average molar liquid density $\left(\mathrm{lb}-\mathrm{mol} / \mathrm{ft}^{3}\right), \mathrm{x}_{\mathrm{i}}$ is $\mathrm{SO}_{2} \mathrm{~mol}$ fraction in inlet liquid, $x_{0}$ is $\mathrm{SO}_{2}$ mol fraction in outlet liquid, and $x^{*}$ is the mole fraction of $\mathrm{SO}_{2}$ in the liquid phase in equilibrium with the gas phase.

The average liquid and gas flow rates per unit area, L' and $G^{\prime}$, are calculated from the inner and outer cross-sectional areas of rotating bed.

$$
L^{\prime}=L_{i}+L_{0} / 2
$$

$$
G^{\prime}=G_{i}+G_{0} / 2
$$

For liquid, $L_{i}=L / A_{i}$

where

$$
A_{i}=2 \pi r_{i} d
$$

$$
L_{0}=L / A_{0}
$$

$$
A_{0}=2 \pi r_{0} d
$$

Here, $A_{i}$ and $A_{0}$ are inner and outer cross-sectional areas, $r_{i}$ and $r_{0}$ are inner and outer radii of packed bed.

In rotating packed bed, $\mathrm{HTU}$ is defined as:

$$
H T U=\left(r_{0}-r_{i}\right) / N T U
$$

NTU can be found by measuring gas inlet and outlet $\mathrm{SO}_{2}$ concentrations and then by calculating hypothetical liquid concentrations in equilibrium with the gas phase at the inlet and outlet of the rotor from the equilibrium relation in equation (22) to be used in equation (25). Since packing depth, $Z\left(r_{0}-r_{i}\right)$ in 
rotating bed, and NTU are known, overall liquid-phase volumetric mass-transfer coefficient, $K_{L} a$, can de determined by equation (33)

$$
K_{L} a=\frac{L}{H T U A P}=\frac{L}{H T U P}
$$

Similarly, the rate of solute transferred from the gas phase can be expressed in terms of $K_{\sigma^{a}}$ and a logarithmic mean driving force.

$$
G\left(y_{T}-y_{0}\right)=K_{C^{a}} A Z P_{T}\left(y-y^{*}\right)_{L M}
$$

where

$$
\begin{aligned}
& \left(y-y^{*}\right) L M=\frac{\left(y-y^{*}\right) i-\left(y-y^{*}\right) e}{\ln \left(y-y^{*}\right) i} \\
& \left(y-y^{*}\right)_{0}
\end{aligned}
$$

Thus, packing depth:

$$
Z=\frac{G}{K_{C^{a}} A P_{T}} \frac{\left(y-y_{0}\right)}{\left(y-y^{*}\right)_{L M}}
$$

where

$$
H T U=\frac{G}{K_{G^{a}} A P_{T}}=\frac{G^{\prime}}{K_{C^{a}} P_{T}}
$$

and

$$
N T U=\frac{\left(y-y_{0}\right)}{\left(y-y^{*}\right)_{1}-\left(y-y^{*}\right)_{0}} \ln \frac{\left(y-y^{*}\right)_{j}}{\left(y-y^{*}\right)_{0}}
$$

Here, $K_{C} a$ is overall gas-phase volumetric mass-transfer coefficient 
(lb-mol/h.ft $\cdot a t m), y_{i}$ is $\mathrm{SO}_{2}$ mol fraction in gas inlet, $y_{0}$ is $\mathrm{SO}_{2}$ mol fraction in gas outlet, and $\mathrm{y}^{*}$ is $\mathrm{SO}_{2}$ mol fraction in gas phase that would be in equilibrium with the liquid phase.

In a similar manner, HTU in equation (37) may be obtain with a known packing depthi, $Z$, by using equation (32). NTU for gas phase can be estimated by equation (38), once $\mathrm{SO}_{2}$ concentrations in gas phase at the inlet and outlet of rotor and also the $\mathrm{SO}_{2}$ concentration in gas phase which is in equilibrium with the liquid phase are determined. Overall gas-phase volumetric mass-transfer coefficient, $K_{G^{a}}$, can be determined from equation (39).

$$
K_{G^{a}}=\frac{G^{\prime}}{H T U P_{T}}
$$

The relationship between $K_{L} a$ and $K_{C} a$ is expressed by:

$$
\frac{1}{K_{L} a}=\frac{1}{k_{L} a}+\frac{H}{k_{C^{a}}}=\frac{H^{\prime}}{K_{C^{a}}}
$$

Absorption of $\mathrm{SO}_{2}$ in wat?r has been studied by Whitney and Vivian [13] and Pearson et al. [14]. In their: studies it is shown that both liquid and gas film resistances exert an appreciable effect and absorption becomes both liquid and gas film controlled.

The equations in this section, are going to be used in determining $\mathrm{K}_{\mathrm{L}} \mathrm{a}$ and $\mathrm{K}_{\mathrm{C}}$ for $\mathrm{SO}_{2}$-Air-Water system in the rotating packed bed with a countercurrent design. 


\section{3. Chemistry and Absorption-Rate Steps:}

In the $\mathrm{SO}_{2}$ absorption, a portion of sulfur dioxide which is dissolved in water ionizes.

$\mathrm{SO}_{2}(\mathrm{~g}) \longleftrightarrow=\mathrm{SO}_{2}(\mathrm{aq})$

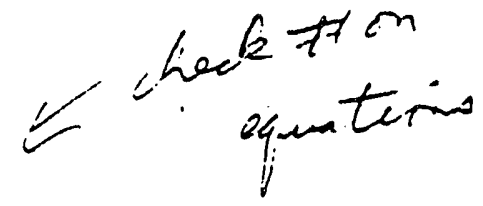

$\mathrm{SO}_{2}(\mathrm{aq})+\mathrm{H}_{2} \mathrm{O} \Leftrightarrow=\mathrm{H}^{+}+\mathrm{HSO}_{3}-$

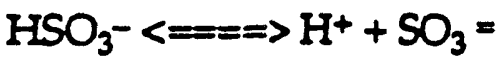

The absorptivity of sulfur dioxide in an aqueous system can be increased by reducing the hydrogen ion concentration or by removing $\mathrm{HSO}_{3}-$ or $\mathrm{SO}_{3}=$. When lime used, the main reaction steps may be summarized as follows: lime dissolution,

$\mathrm{Ca}\left(\mathrm{OH}_{2}(\mathrm{~s}) \Longleftrightarrow \mathrm{Ca}\left(\mathrm{OH}_{2}\right.\right.$ (aq)

$\mathrm{Ca}\left(\mathrm{OH}_{2}(\mathrm{aq}) \Leftrightarrow=\Rightarrow \mathrm{Ca}^{++}+2 \mathrm{OH}^{-}\right.$

reaction with dissolved $\mathrm{SO}_{2}$

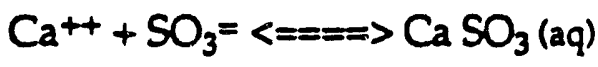

$\mathrm{CaSO}_{3}(\mathrm{aq})+1 / 2 \mathrm{H}_{2} \mathrm{O} \Leftrightarrow=\Rightarrow \mathrm{CaSO}_{3} \cdot 1 / 2 \mathrm{H}_{2} \mathrm{O}(\mathrm{s})$

oxidation,

$\mathrm{HSO}_{3}-+1 / 2 \mathrm{O}_{2}<=\Rightarrow \Rightarrow \mathrm{SO}_{4}=+\mathrm{H}^{+}$

$\mathrm{Ca}++\mathrm{SO}_{4}=\Longleftrightarrow \mathrm{CaSO}_{4}(2 \mathrm{q})$

$\mathrm{Ca} \mathrm{SO}_{4}(\mathrm{aq})+2 \mathrm{H}_{2} \mathrm{O} \Longleftrightarrow \mathrm{Ca} \mathrm{SO}_{4} \cdot 2 \mathrm{H}_{2} \mathrm{O}(\mathrm{s})$

Equilibrium species in $\mathrm{SO}_{2}$ absorption versus $\mathrm{pH}$ is shown in Figure 5.

In the removal of $\mathrm{SO}_{2}$ from tue gases by lime slurry, the following 


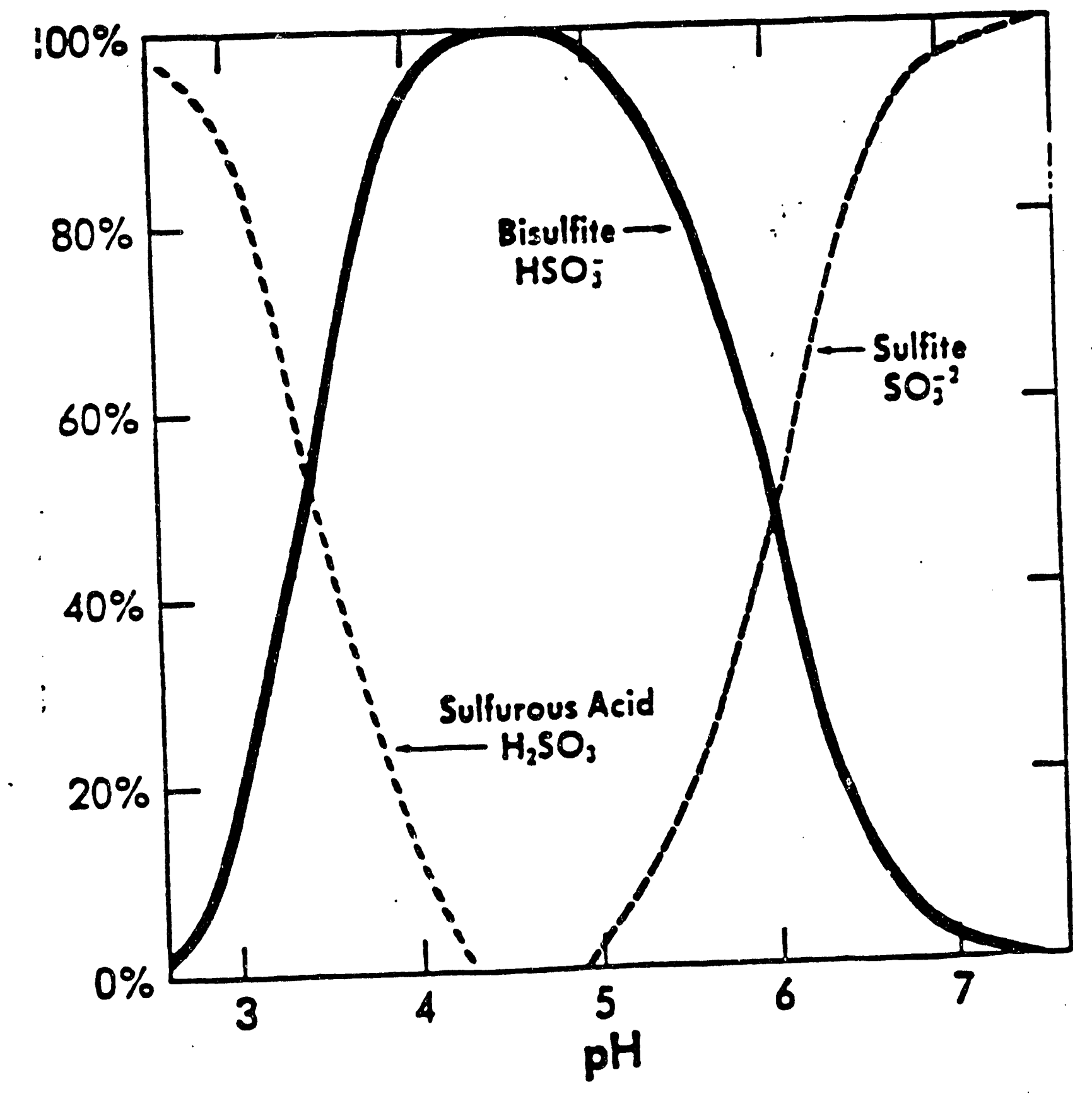

Figure 5. $\mathrm{SO}_{2}$ Absorption species versus $\mathrm{pH}$ 
individual steps take place.

1. Diffusion of $\mathrm{SO}_{2}$ to the gas/liquid interface through the gas film

2 Discolution of $\mathrm{SO}_{2}$ in water at the interface

3. Ionization of dissolved $\mathrm{SO}_{2}$ to $\mathrm{H}^{+}$and $\mathrm{HSO}_{3}-$

4. Dissociation of $\mathrm{HSO}_{3}-$ to form $\mathrm{SO}_{3}=$

5. Diffusion of $\mathrm{HSO}_{3}-, \mathrm{H}^{+}$, and $\mathrm{SO}_{3}=$ ions from the liquid/gas interface through liquid film into the liquid interior

6. Dissolving of $\mathrm{Ca}(\mathrm{OH})_{2}$

7. Ionization of dissolved $\mathrm{Ca}(\mathrm{OH})_{2}$ to $\mathrm{Ca}^{++}$and $\mathrm{OH}^{-}$ions

8. Reaction of $\mathrm{Ca}^{++}$with $\mathrm{SO}_{3}=$ to form $\mathrm{Ca} \mathrm{SO}_{3}$

9. Precipitation of $\mathrm{Ca} \mathrm{SO}_{3} \cdot 1 / 2 \mathrm{H}_{2} \mathrm{O}$

10. Oxidation of $\mathrm{SO}_{3}=$ to $\mathrm{SO}_{4}-$

11. Reaction of $\mathrm{Ca}^{++}$with $\mathrm{SO}_{4}=$ to form $\mathrm{CaSO}_{4}$

12. Precipitation of $\mathrm{Ca} \mathrm{SO}_{4} \cdot 2 \mathrm{H}_{2} \mathrm{O}$

Since ionic reactions are very fast, rate controlling steps can be step 1, gas phase mass transfer, step 5, liquid phase mass transfer and step 6, dissolution of $\mathrm{Ca}(\mathrm{OH})_{2}$. Because dissolution rate $\mathrm{Ca}(\mathrm{OH})_{2}$ is fast enough and $\mathrm{Ca}(\mathrm{OH})_{2}$ has high surface area, step 6 is not controlling. High solubility of $\mathrm{SO}_{2}$ in lime slurry due to chemical reaction makes step 5, liquid phase transfer, have little effect. Th a absorption of $\mathrm{SO}_{2}$ in lime slurry becomes gas phase mass transfer limiting. 


\subsection{Calculation of $\mathrm{K}_{\mathrm{G}^{2}}$ for $\mathrm{SO}_{2}$ Air-Lime system in Rotating Bed:}

$\mathrm{SO}_{2}$ being removed from the gas phase can be expressed in terms of $\mathrm{K}_{\mathbf{G}^{\mathrm{a}}}$

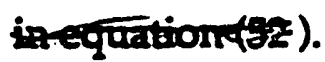

$$
N=G\left(y_{i}-y_{0}\right)=K_{\sigma^{2}} A Z P_{T}\left(y-y^{*}\right) / M .
$$

where

$$
\left(y-y^{*}\right) L M=\frac{\left(y-y^{*}\right)_{i-}\left(y-y^{*}\right)_{0}}{\ln \frac{\left(y-y^{*}\right)_{i}}{\left(y-y^{*}\right)_{0}}}
$$

$\mathrm{Ca}(\mathrm{OH})_{2}$ reacts rapidly with $\mathrm{SO}_{2}$ absorbed in the liquid phase. Therefore, the vapor pressure above the liquid phase is eliminated or substantially reduced, causing Henry's Law constant, $H$, to approach to zero. In this case, mass transfer driving force, $\left(p-p^{*}\right)$, is maximized. Assuming $y^{*}$ to be zero at the inlet and outlet of the rotor, equation (52) is reduced to the following term.

$$
G\left(y_{i}-y_{0}\right)=K_{C^{a}} \text { A } Z P_{T} \frac{\left(y_{i}-y_{0}\right)}{\ln \left(y_{i} / y_{0}\right)}
$$

Packing depth, $Z$, is:

$$
Z=\frac{G}{K_{C^{a}} A P_{T}} \ln \frac{y i}{\text { yo }}
$$

where $\quad H T U=\frac{G}{K_{C^{a} A P_{T}}}=\frac{G^{\prime}}{K_{G^{a} P_{T}}}$

(56) and $N T U=\ln \frac{y i}{y_{0}}$

Here, $\mathrm{N}$ is transfer rate of $\mathrm{SO}_{2}$ from the gas phase to liquid phase (lt-mol/h), G 
is molar gas flow rate $(\mathrm{lb}-\mathrm{mol} / \mathrm{h}), G$ is the average molar gas flow rate based on inner and outer cross-sectional area of rotating bed $\left(a b-m o l / f t^{2} \cdot h\right), y_{i}$ and Yo are the $\mathrm{SO}_{2}$ concentrations at the inlet and outlet gas, respectively.

By measuring the inlet and outlet $\mathrm{SO}_{2}$ concentrations in the gas phase for a known packing depth, HIU and then $K_{\sigma^{a}}$ can be calculated according to the equation below.

$$
H T U=\frac{Z}{N I U}=\frac{G^{\prime}}{K_{C^{a}} P_{T}}
$$

In equation (58) $\mathrm{K}_{\mathrm{G}^{\mathrm{a}}}$ is the overall gas-phase volumetric mass-transfer coefficient $\left(\mathrm{lb}-\mathrm{mol} / \mathrm{ft}^{2} \cdot \mathrm{h} \cdot \mathrm{atm}\right), \mathrm{a}$ is the interfacial area per unit volum of packing $\left(\mathrm{ft}^{2} / \mathrm{ft}^{3}\right)$.

For $\mathrm{SO}_{2}$-Air-Lime system in which $\mathrm{H}$ is very large, overall and individual mass-transfer cofficients $K_{-} a$ and $k_{C} a$ are equal to eachother according to equation (13).

4. 5. The Effect of High Gravitational Field on Absorption with compared to

\section{Conventional Packed Tower.}

The packed towers which are usually filled with randomly oriented packing material, are extensively used for absorption operations to provide a significant gas-liquid contact area. The liquiid phase is introduced over the top of the packed bed. The liquid trickles down and wets the packing which has a large surface area. The solute-containing gas phase enters the column below 
the packing at the bottom of the tower and flows upward through the packing elements countercurrent to the liquid flow and exits at the top of the tower. While liquid flows downward under the influence of gravity, the gas which flows upward must overcome the resistance caused by the tower packing elements.

The rate of mass transfer from the liquid to the gas phase can be increased by increasing the gas and liquid flow rates through the tower. The increased flow rates cause convection and turbulent mixing to be increased in both gas and liquid phases. However, an upper limit called flooding region exists for the gas flow rates in which the entrainment of liquid by gas is begone. Most packed towers is operated below the flooding region, resulting in a limitation in the mass transfer rate. In a packed tower relation for pressure drop and flooding is given by Sherwood correlation shown in Figure 6. In equations (24), (37) and (56), HTU is a function of gas and liquid flow rates, the characteristics of packing, gas and liquid-phase mass-transfer coefficients. NTU depends on concentration of solute in the inlet and outlet and phase equilibria properties of solute in equations (25), (38), (57)

When absorption is carried out in the high gravitational field by using rotating packed bed, higher gas and liquid flow rates, and higher surface area of packing can be used without flooding of the bed. Assuming that Sherwood equation is conservative in the rotating bed, this effect can be observed since gravitational constant, $g$, is included in the denominator.

Volumetric mass transfer coefficients are enhanced, resulting from the 


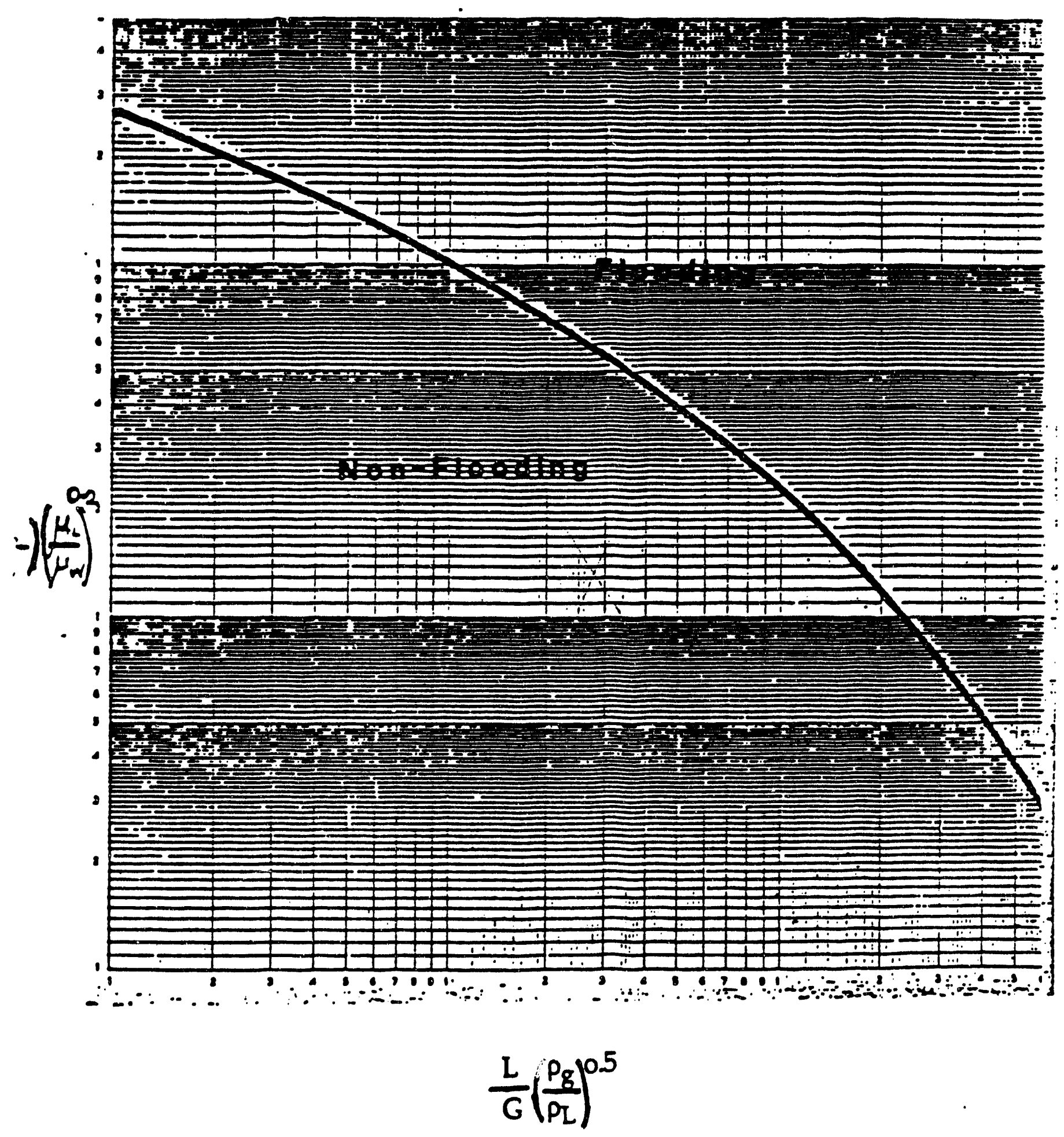

Figure 6. Sherwood Correlation 
use of high gas flow rate and high surface area. Other contributing factors to the higher coefficients are an increase in the effective gas-liquid contacting efficiency, $a_{e} / a$, and an increase in the liquid-phase mass-transfer coefficient, $k_{L}$. Increase in overall mass-transfer coefficients and interfacial area of packing reduces HTU. As a result, a significant reduction in absorber size can be achieved. 


\section{EXPERIMENTAL RESULTS AND DISCUSSIONS:}

The overall volumetric mass-transfer coefficients are determined in rotating packed bed for $\mathrm{SO}_{2}$ absorption in water and lime slurry. The effect of acceleration field, the liquid and gas flowrates on the overall volumetric mass-transfer coefficients were investigated for both system. Runs were repeated in the same conditions to determine experimental error and reproducibility. Experimental results are summarized in Appendix.

\section{A. $\mathrm{SO}_{2}$-Air-Water System:}

\section{A.1. Effect of Gravity:}

Experimental runs were made in constant gas flowrates of 20 and $40 \mathrm{acfm}$ and liquid flowrates of $2,3,4,5 \mathrm{gpm}$. $\mathrm{SO}_{2}$ inlet concentration in the gas mixture is kept constant at $1000 \mathrm{ppm}$. The rotor speed was set at $250,700,850$, $1000,1300,1500 \mathrm{rpm}$ to achieve a centrifugal acceleration of $124,97.3,143.5$, $198.7,335.8$, and $447 \mathrm{~g}$ respectively (arithmetical average of gravity based on the inner and outer radius of the rotor). Figure 7 depicts the influence of gravity on calculated $K_{G}$ a for different liquid flowrates. Overall volumetric mass-transfer coefficients increase logarithmically with the gravity. As shorm in Figure 8, at gas flowrate of $40 \mathrm{acfm}$ and liquid flowrate of $3 \mathrm{gpm}$, lineer regression analysis of the logarithm of the $K_{C^{a}}$ vs \#g data yields $K_{C^{2}} \alpha$ $G^{0.37-0.40}$ in agreement with Dudukovic and Vivian but regression coefficient for the exponent is poir $(0.86-0.92)$ indicating considerable scatter. It should be noted that agreement with Dudokovic's result is only valid for liquid 
S02-Alr-Water

$G=20 \mathrm{acfm}, y l=1000 \mathrm{ppm}$

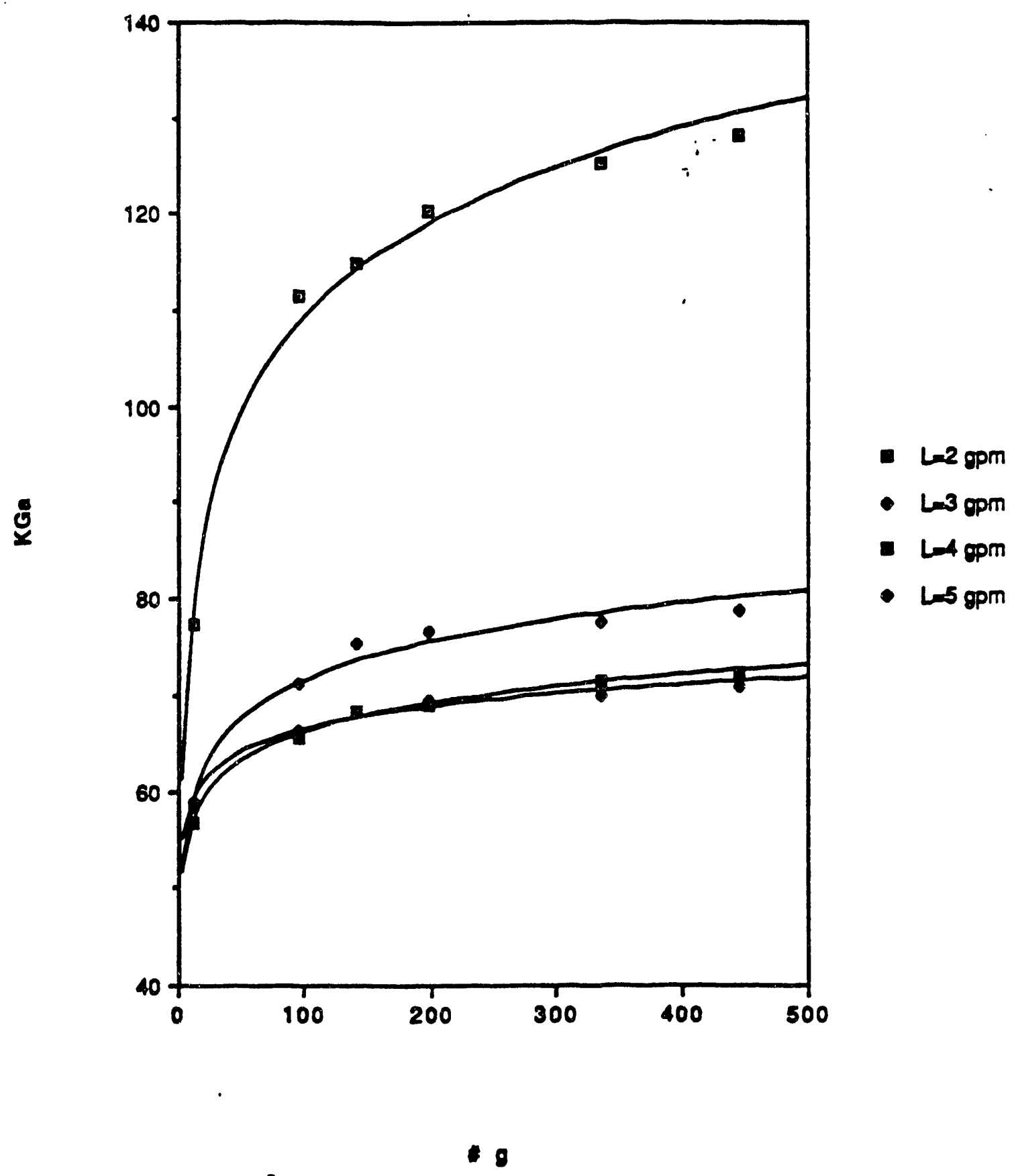

Figure 7 
$\mathrm{SO}_{2}-$ Air-Water System
$\mathrm{G}=40 \mathrm{acfm} \quad \mathrm{y}_{1}=1000 \mathrm{ppm}$

600

$\stackrel{\mathbb{8}}{\check{Y}}$

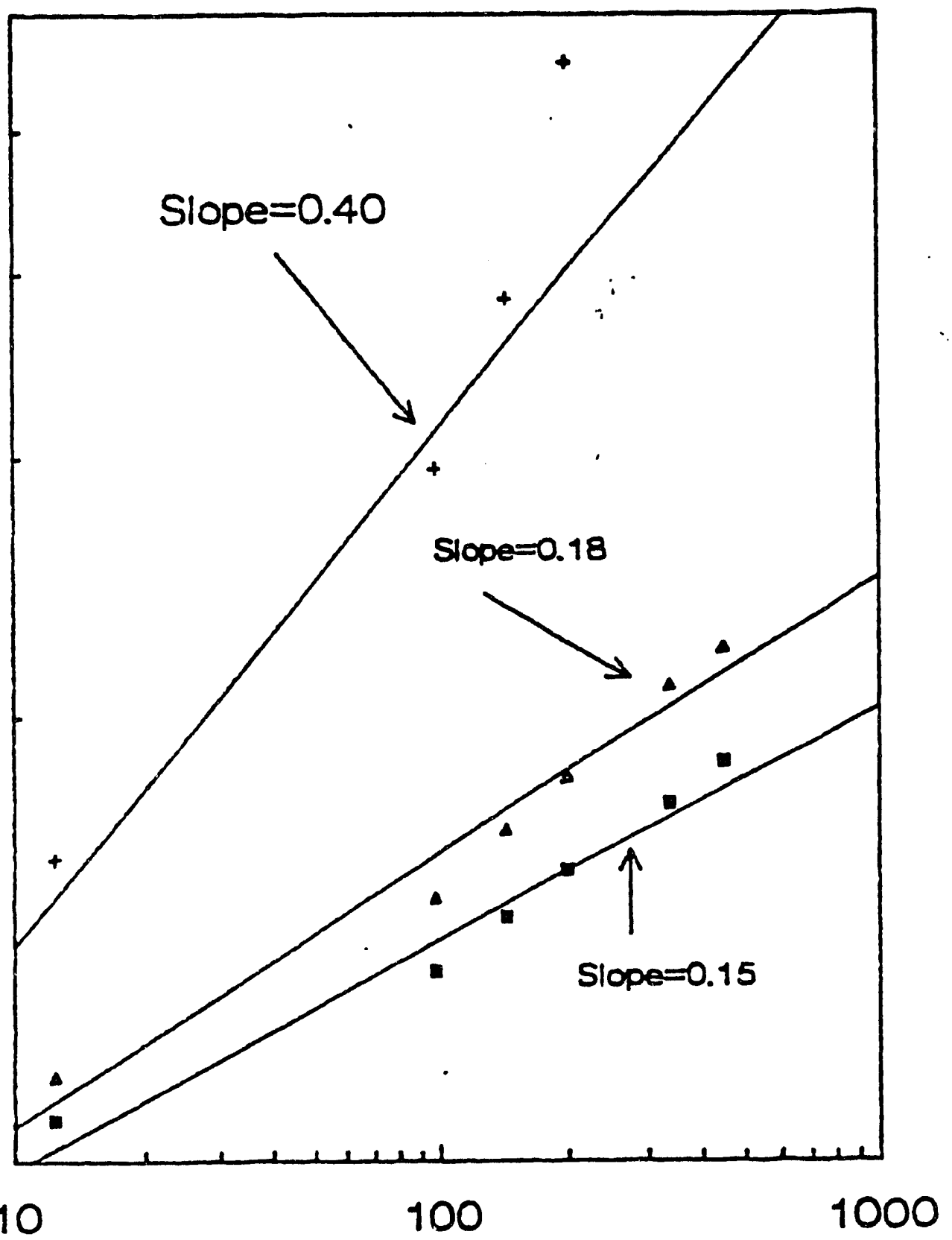

\# g

$+L=3 \mathrm{gom} \Delta \quad \mathrm{L}=4 \mathrm{amm} \quad L=5 \mathrm{com}$

Figure 8 
flowrate of 3 gpm.

5.2. Effect of Liquid Flowrate

At constant gas flowrate of $40 \mathrm{actm}$ and $20 \mathrm{acfm}$, the influence of liquid flowrates of $1-5$ gpm is investigated as shown in Figure 9 and Figure 10. The slopes of the best line drawn through these data vary considerably with liquid flowrates. Slopes vary between $0.4-0.10$ at $\mathrm{G}=40 \mathrm{acfm}$, and $0.14-0.053$ at $\mathrm{G}=20$ acfm.

\section{A.3. Effect of Gas Flowrate:}

At constant liquid flowrates, gas flowrates are changed from $20 \mathrm{acfm}$ to 40 acfm. For higher gas flowrates, higher volumetric mass-transfer coefficients are obtained. The influence of gravity on $K_{C^{a}}$ at lower gas flowrates was smaller. The same trend are observed for different liquid flowrates. Figure 11 shows the effect of gas flowrate on the influence of gravity for constant liquid flowrate of $5 \mathrm{gpm}$.

\section{A.4. Discussion:}

The observation of lowering gravity effect for higher liquid flowrates may be explained as follows. For liquid-phase mass-transfer controlling systems, the improvement of volumetric mass-transfer coefficient is achieved as liquid film gets thinner in high gravity. The chance of having thin liquid film on the surface of packing is reduced at higher liquid flowrates. Also higher gas flowrates help liquid be dispersed better and consequently reduce liquid film thickness. It should be noted that $\mathrm{SO}_{2}$ absorption in water is liquid 
$\mathrm{SO}_{2}$-Air-Water System
$\mathrm{G}=4$ Oacfm $y_{1}=1000 \mathrm{ppm}$

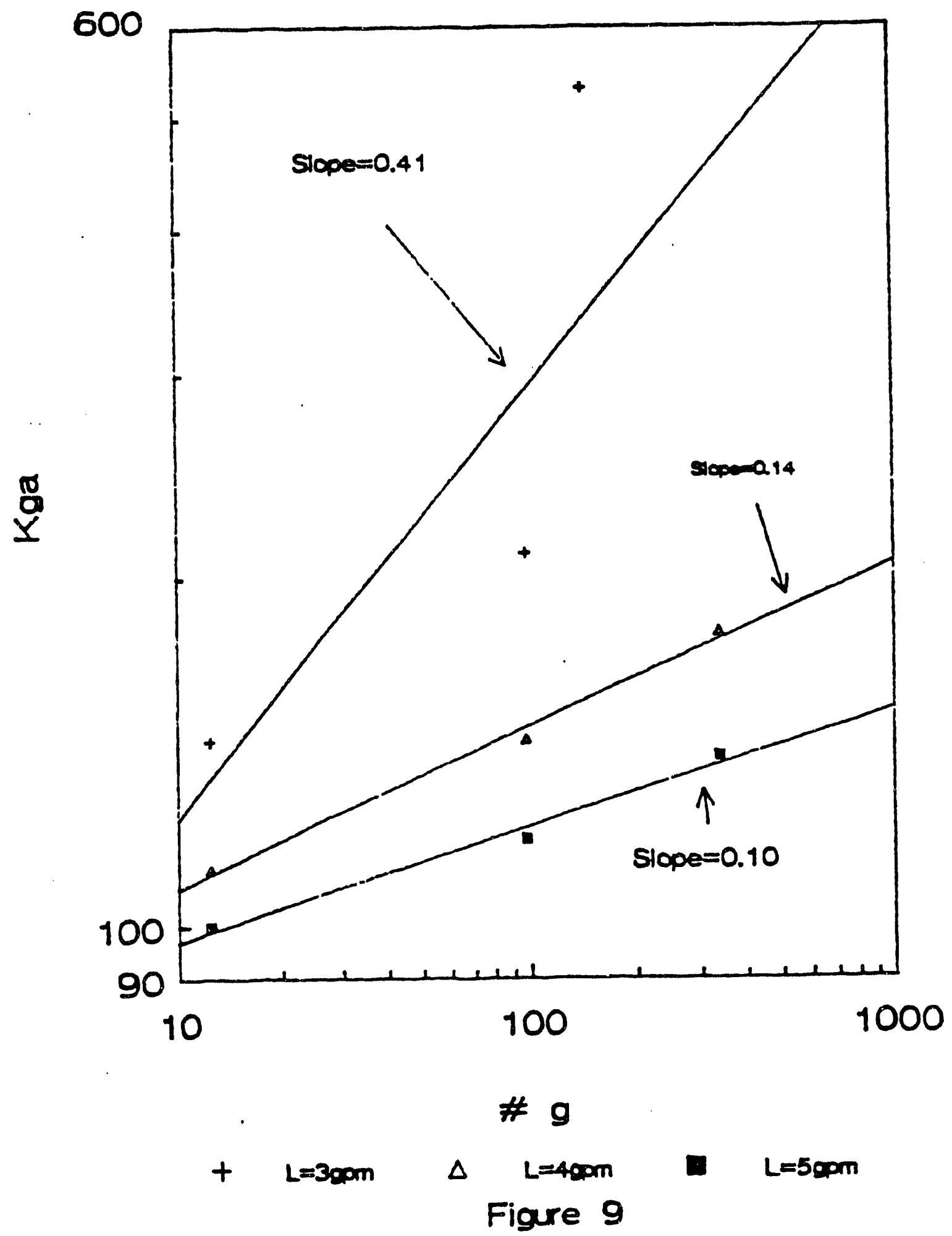


$\mathrm{SO}_{2}$-Air-Water System
$G=20$ acfm $y_{1}=1000 p p m$

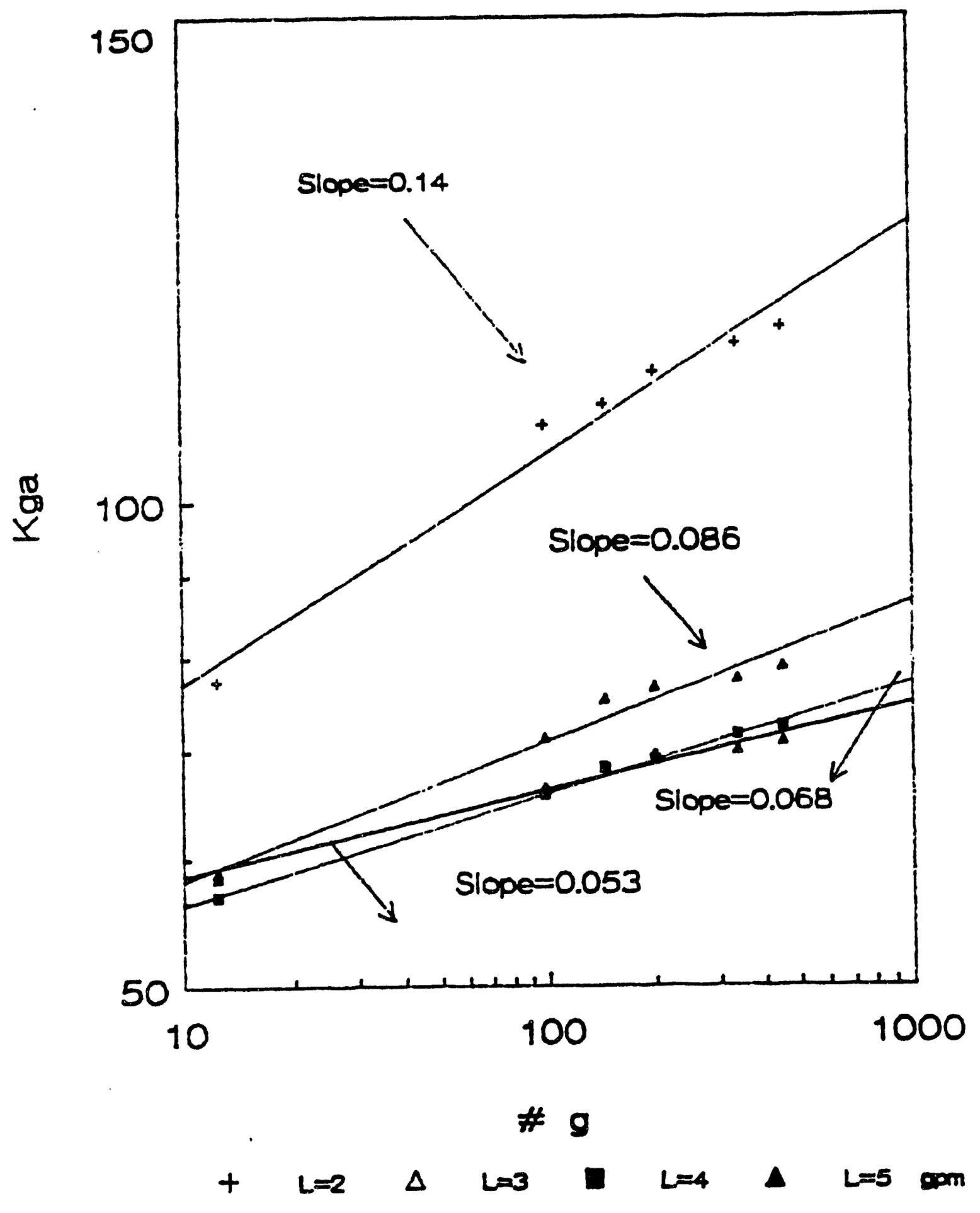

Figre 10 


\section{$\mathrm{SO}_{2}$-Air-Water System $L=5 \mathrm{gpm} \quad y_{1}=1000 \mathrm{ppm}$}

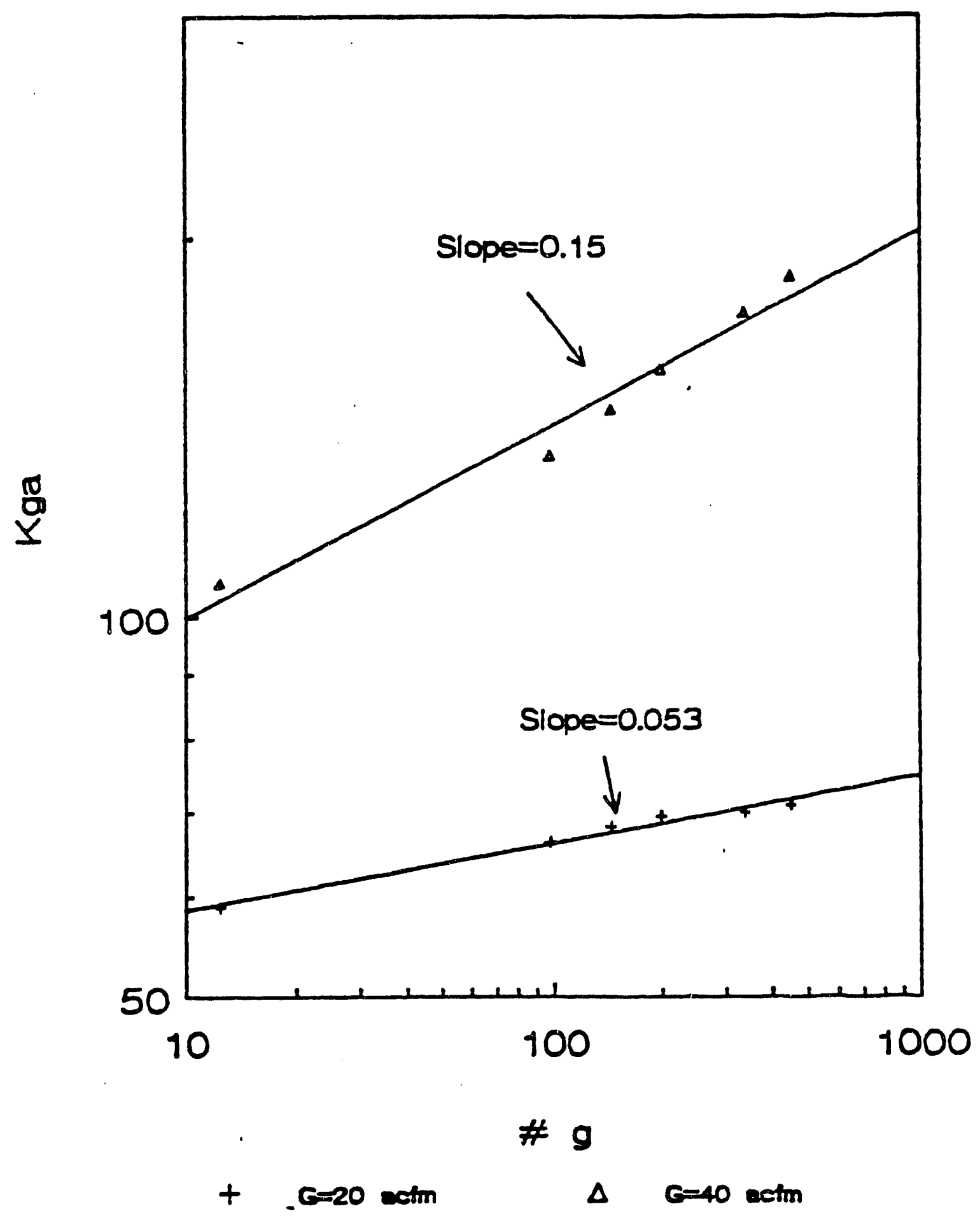

Fiare 11 
and gas-phase mass-transfer limiting, therefore calculated $K_{C} a$ contains liquid and gas-phase effects together.

\section{B. $\mathrm{SO}_{2}$-Air-Lime System:}

Tests were also performed for $\mathrm{SO}_{2}$ absorption in lime slurries. Hydrated lime cointaining $93 \% \mathrm{Ca}\left(\mathrm{OH}_{2}\right.$ and $2 \% \mathrm{Mg}(\mathrm{OH})_{2}$ is used. Stoichiometric ratio of 1.3 is used to provide $30 \%$ excess $\mathrm{Ca}(\mathrm{OH})_{2}$ in slurry. In this conditions gas-phase controls mass transfer. Overall volumetric mass-transfer coefficient, $K_{G} a$, is equal to individual gas-phase mass-transfer coefficient, $k_{C^{2}}$

\section{B.1 Effect of Gravity:}

Liquid and gas flowrates were held constant, while the rotor speed was set at $250,400,500,700,800,1000,1300$, and $1500 \mathrm{rpm}$ to achieve a centrifugal acceleration of $124,31.7,49.6,97.3,143.5,198.7,335.8$ and 447 g, respectively (g is calculated as the average centrifugal acceleration based on the inner and outer radius of the rotor). Figure 12 and 13 show the effect of gravity on $\mathrm{K}_{\mathrm{C}^{a}}$ at $L=2 \mathrm{gpm}$ and $G=40 \mathrm{acfm}$ : for two runs performed in the same conditions. $K_{G^{a}}$ values is observed to be logarithmically increasing with increasing numbers of gravity. Linear regression analysis of the logarithm of $\mathrm{K}_{\mathrm{G}^{\mathrm{a}}}$ vs \# $\mathrm{g}$

yields as $K_{C^{2}} \propto g^{0.0030003}$ in Figure 14 and 15.

\section{B.2. Effect of Liquid Flowrate:}

The $K_{C}$ dependency on acceleration fields was determined at liquid 
SO2-AIr-LIme

$G=40$ acfm, $L=2 \mathrm{gpm}, y l=1000 \mathrm{ppm}$

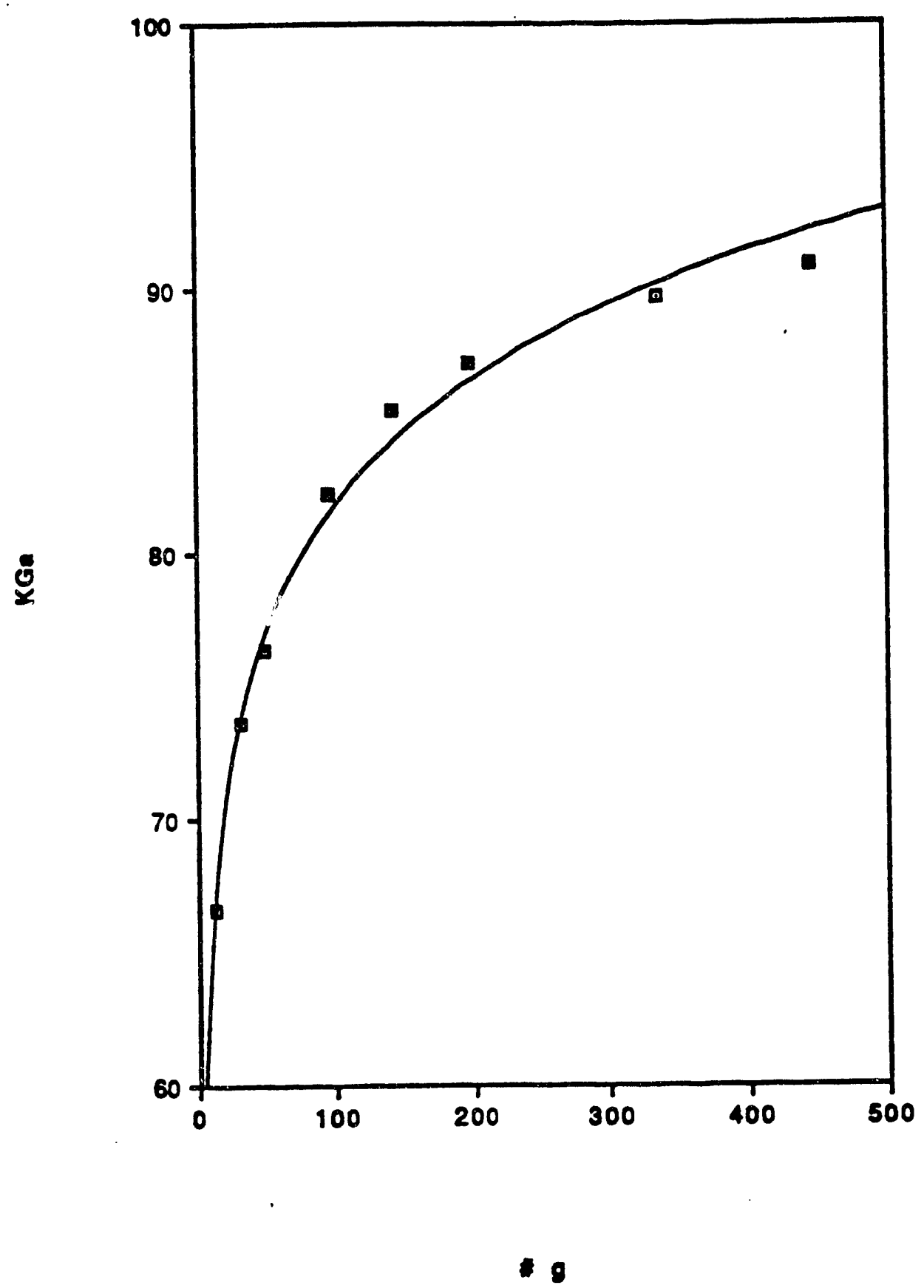

Figure 12 


\section{SO2-Alr-Lime}

$G=40$ acfm, $L=2 \mathrm{gpm}, y l=1000 \mathrm{ppm}$

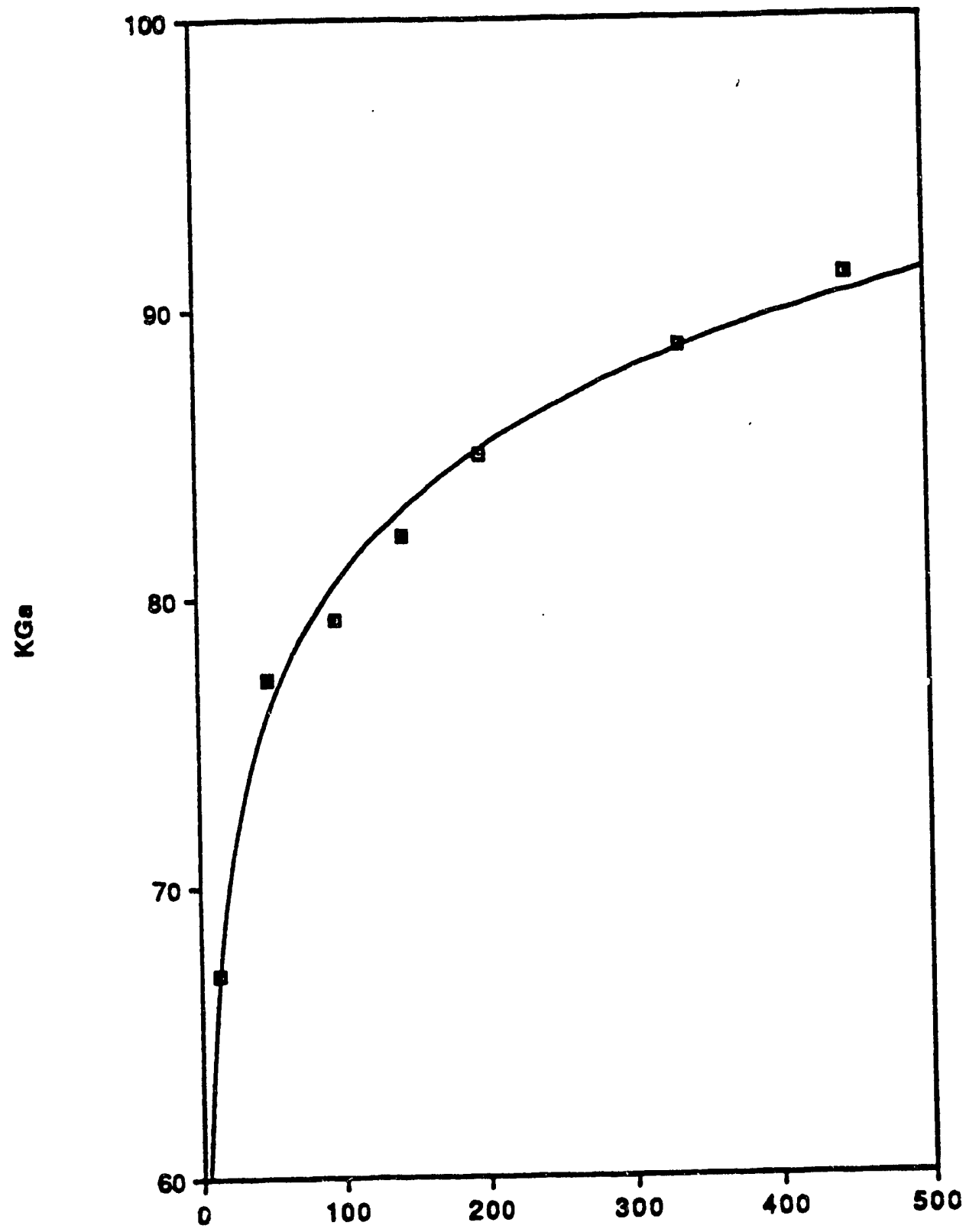

Figure 13 

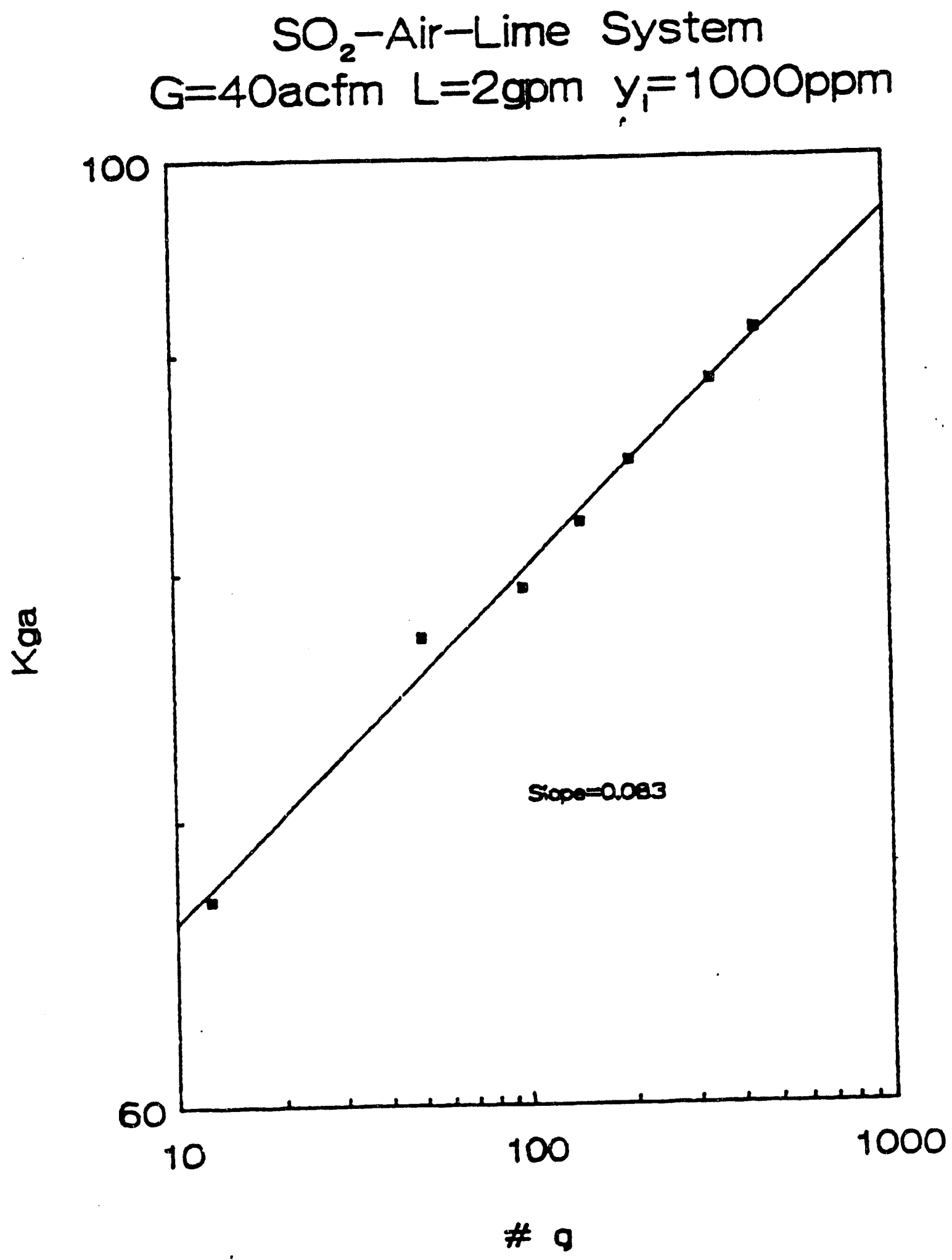

Figure 14 


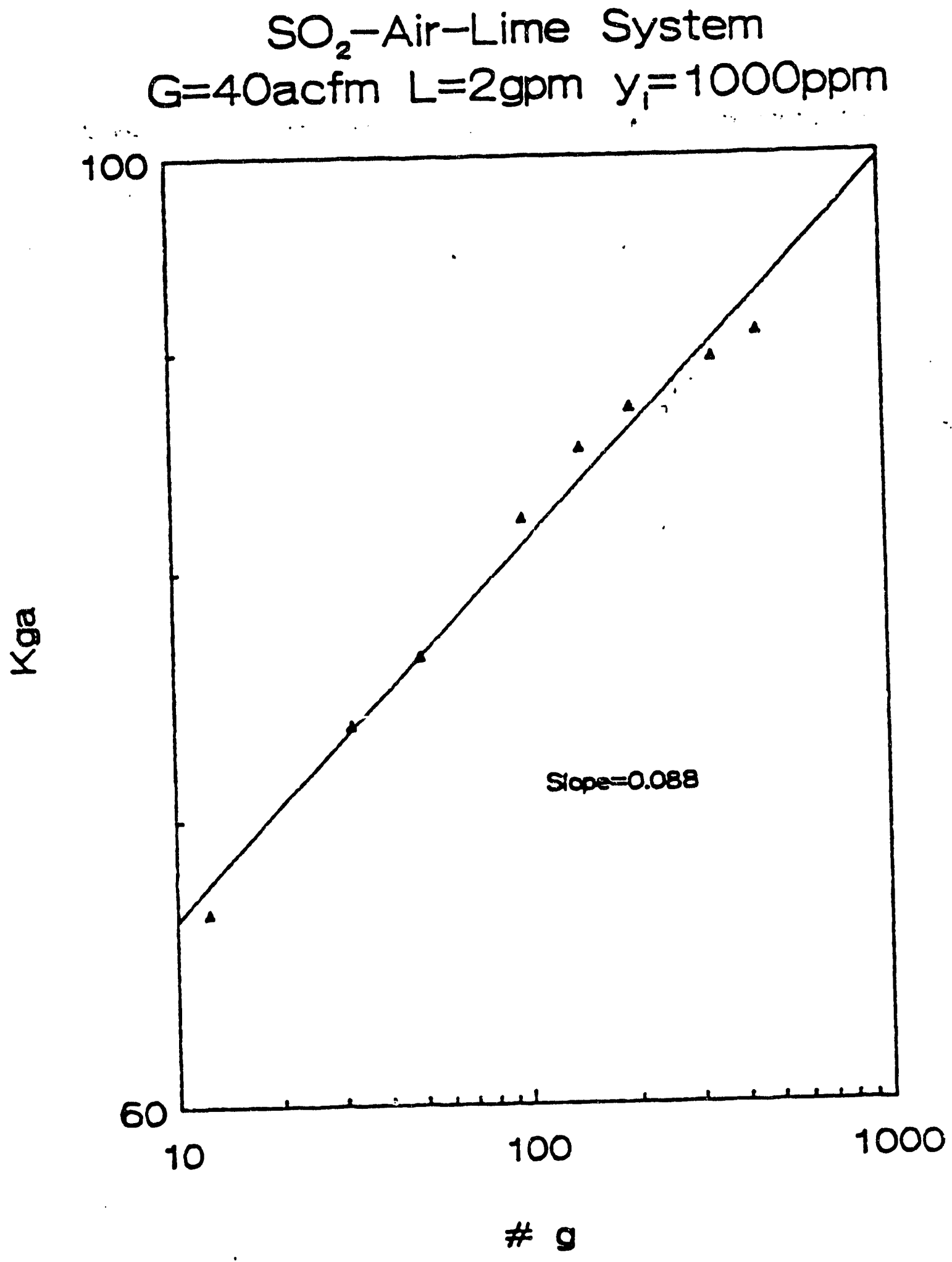

Figuie 15 
flowrates of 1 and $2 \mathrm{gpm}$, while gas flowrates are held constant at 55 acfon The slopes of the best line drawn through data, using least square technique are 0.115-0.09 for $1 \mathrm{gpm}$ and 0.078-0.073 for $2 \mathrm{gpm}$ in Figure 16. Figure 17 depicts the effect of liquid flowrate at $G=40$ acfm, giving slopes of 0.105-0.088. It turns out to be that the liquid flowrates does not affect the power dependency of $K_{C^{a}}$ on gravitational field. It can be recognized that differencies between slopes are within the range of experimental error.

\section{B.3. Effect of Gas Flowrate:}

Liquid flow rate of $1 \mathrm{gpm}$ is kept constant, and gas flowrates of 40 and 55 acfm are used. As Figure 18 illustrates, for higher gas flowrates, higher $K_{C^{a}}$ values are obtained but slopes do not change $(0.115-0.105)$ within the range of experimental error. It can be said that gas flowrates does not affect the power dependency of $K_{C^{a}}$ on gravitational field.

\section{B.4. Discussion:}

$\mathrm{SO}_{2}$ absorption in lime slurry is an example for the absorption accompanied by chemical reaction. Since $\mathrm{SO}_{2}$ molecules reacts on the gas-liquid interface, liquid film distance through which $\mathrm{SO}_{2}$ passes are greatly reduced. Therefore, the effect of gravity in terms of reducing the liquid film thickness is less pronounced for gas-phase mass-transfer limiting systems. 


\section{$\mathrm{SO}_{2}$-Air-Lime System \\ $\mathrm{G}=55 \mathrm{acfm} \quad \mathrm{y}_{1}=1000 \mathrm{ppm}$}

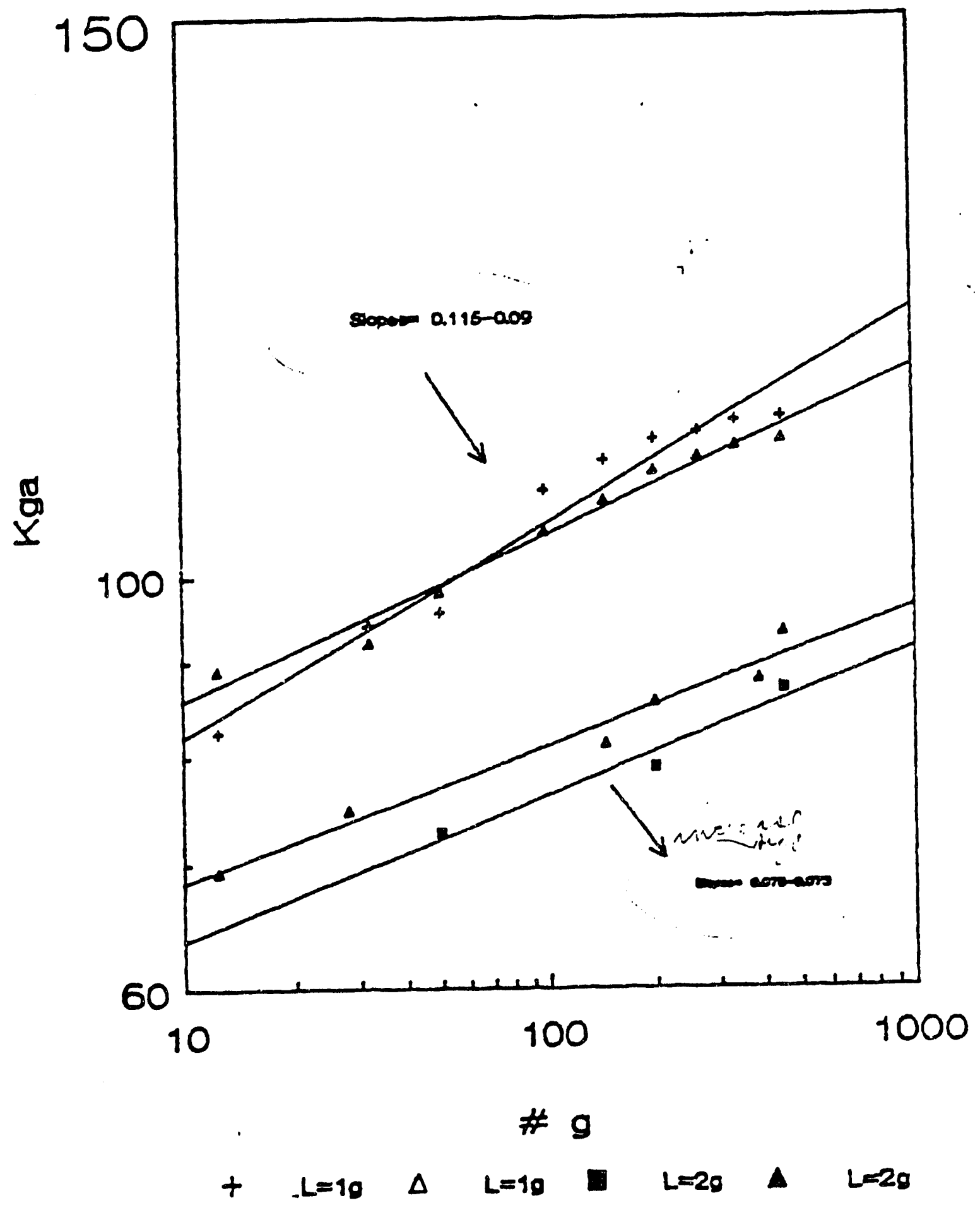

Figure 16 


$$
\begin{aligned}
& \mathrm{SO}_{2} \text {-Air-Lime System } \\
& \mathrm{G}=40 \text { acfm } y_{1}=1000 \text { ppm }
\end{aligned}
$$

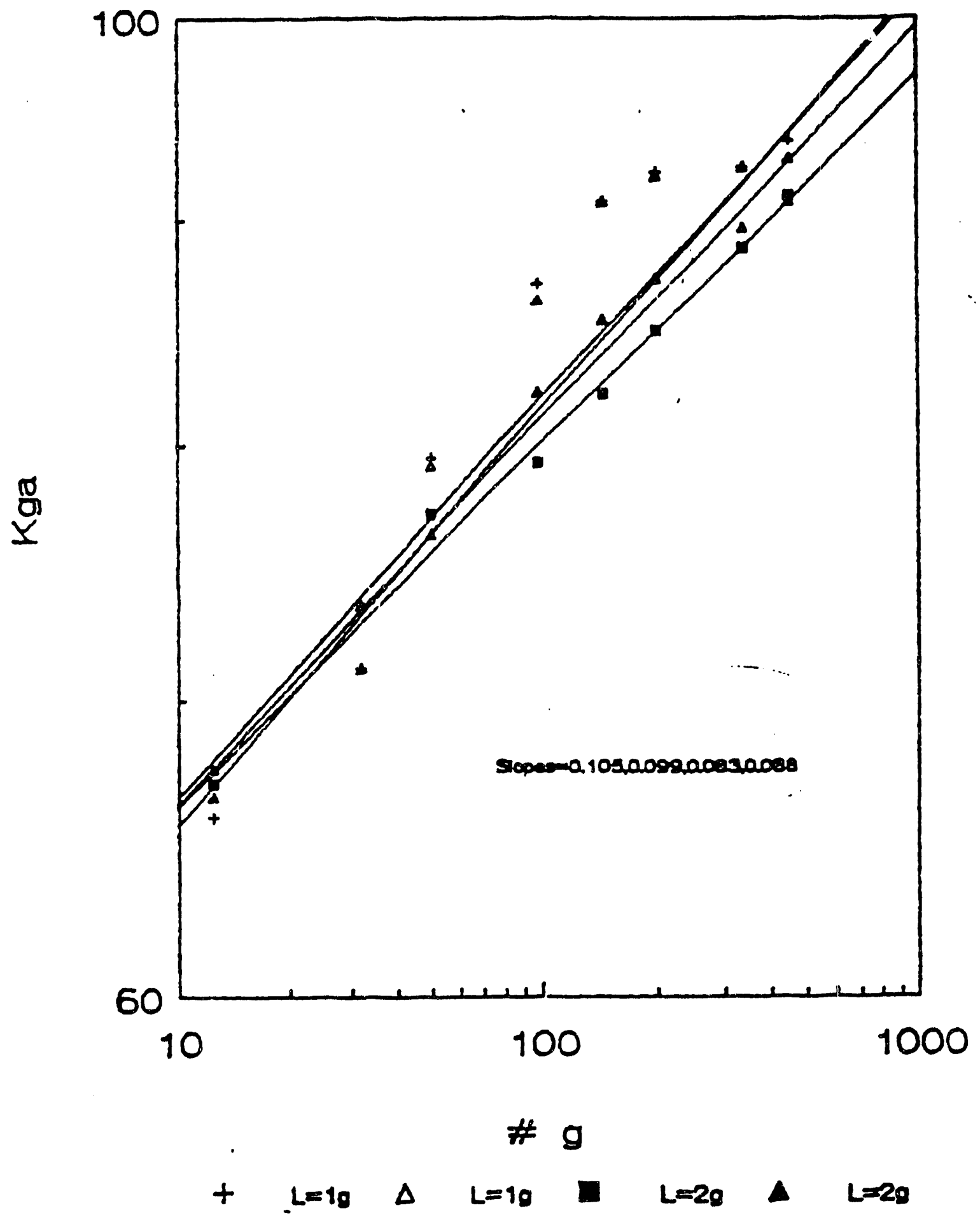

Figure 17 
$\mathrm{SO}_{2}$-Air-Lime System
$L=1 \mathrm{gpm} \quad y_{1}=1000 \mathrm{ppm}$

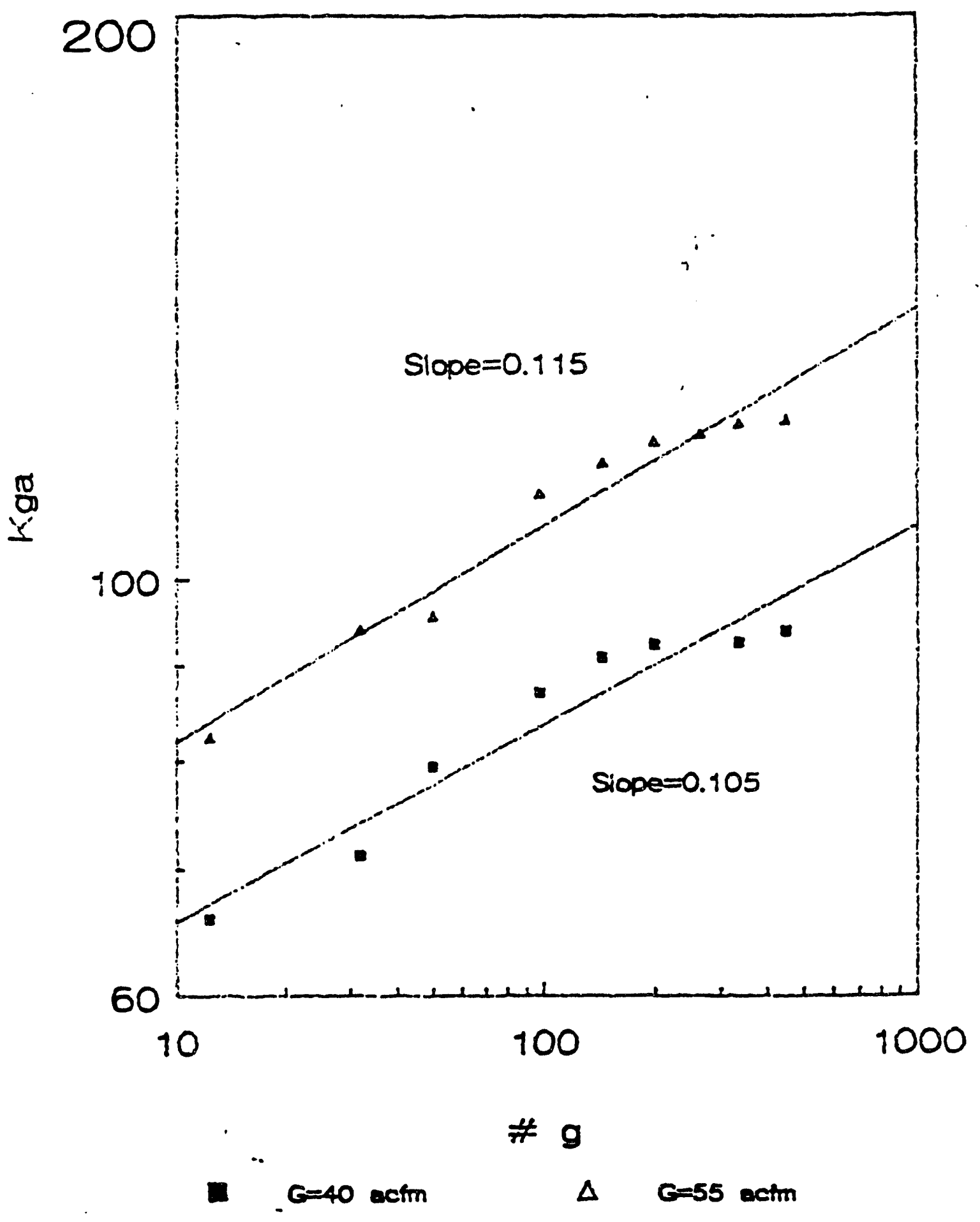

Figure 18 


\section{CONCIUSION:}

For $\mathrm{SO}_{2}$-Air-Water system, the overall volumetric mass-transfer coefficient, $K_{C}$, increases logarithmically with average gravity to a power between $0.40-0.15$. Liquid flowrate has great influence on the gravity effect. The effect of gravity increases as the liquid flowrate decreases. In higher gas flowrates higher $\mathrm{K}_{\mathrm{C}} \mathrm{a}$ values are obtained and gravity effect is increased.

In $\mathrm{SO}_{2}$-Air-Lime system volumetric mass-transfer coefficient, $\mathrm{K}_{\mathrm{C}^{\mathrm{a}}}$, is

found to increase logarithmically as $K_{-} a \propto g^{0.08-0.11}$. Gravity effect on $K_{G^{a}} a$ is less pronounced for gas-phase mass-transfer limiting system. The effect of gravity on $K_{C^{a}}$ is not found to be influenced by gas and liquid flowrates.

For both systems, the overall volumetric mass-transfer coefficients obtained in rotating bed are much higher than the corresponding values in conventional packed towers. 


\section{NOMENCLATURE:}

a. effective gas-liquid interfacial area $\left(\mathrm{ft}^{2} / \mathrm{ft}^{3}\right)$

a total packing surface area ( $\left.\mathrm{ft}^{2} / \mathrm{ft}^{3}\right)$

A packing cross-sectional area ( $\mathrm{ft}^{2}$ )

$A_{i}$ inner cross-sectional area of rotating bed ( $\mathrm{ft}^{2}$ )

$A_{0}$ outer cross-sectional area of rotating bed $\left(\mathrm{ft}^{2}\right)$ :

c $\mathrm{SO}_{2}$ concentration in liquid ( $\mathrm{lb}-\mathrm{mol} / \mathrm{ft}^{3}$ )

d diameter (or characteristic dimension) of packing ( $\mathrm{ft}$ )

d width of packed bed ( $f t$ )

D liquid-phase diffusion coefficient $\left(\mathrm{ft}^{2} / \mathrm{h}\right)$

8 gravitational acceleration ( $\mathrm{ft} / \mathrm{sec}^{2}$ )

G molar gas flowrate ( $\mathrm{lb}-\mathrm{mol} / \mathrm{h}$ )

G' average of molar gas flowrates per unit area based on inner and outer cross-sectional area of rotating bed $\left(\mathrm{lb}-\mathrm{mol} / \mathrm{ft}^{2} \cdot \mathrm{h}\right.$ )

$\mathrm{G}_{\mathrm{i}}$ molar gas flowrate per unit area based on inner cross-sectional area of rotating bed (lb-mol $\left./ \mathrm{ft}^{2} \cdot \mathrm{h}\right)$

Go molar gas flowrate per unit area based on outer cross-sectional area of rotating bed $\left(\mathrm{lb}-\mathrm{mol} / \mathrm{ft}^{2} \cdot \mathrm{h}\right)$

h. height of short wetted-wall column ( $f t$ )

H Henry's low constant ( atm/ (ab-mol/ft $\left.\mathrm{ft}^{3}\right)$ )

H' Henry's low constant ( (lb-mol/ft $\left.\left.\mathrm{ft}^{3}\right) / \mathrm{atm}\right)$ 
HIU height of a transfer unit ( $\mathrm{ft}$ )

k gas-phase mass-transfer coefficient ( Ib-mol/ h.ft $\cdot$ aton )

$k_{L} \quad$ liquid-phase mass-transfer coefficient ( $\mathrm{lb}$-mol/h- $\mathrm{ft}^{2} \cdot\left(\mathrm{bb}-\mathrm{mol} / \mathrm{ft}^{3}\right.$ ) )

$$
\text { or }(\mathrm{ft} / \mathrm{h})
$$

$K_{C^{a}}$ overall volumetric gas-phase inass-transfer coefficient

$$
\left(\mathrm{lb}-\mathrm{mol} / \mathrm{h} \cdot \mathrm{ft}^{3} \cdot \mathrm{atm}\right)
$$

$K_{L}$ a overall volumetric liquid-phase mass-transfer coefficient ( $\left.1 / h\right)$

L molar liquid flow rate ( $\mathrm{lb}-\mathrm{mol} / \mathrm{h}$ )

L' average of molar liquid flowrates per unit area based on the inner and outer cross-sectional area of rotating bed ( $\mathrm{lb}-\mathrm{mol} / \mathrm{ft}^{2} \cdot \mathrm{h}$ )

$\mathrm{L}_{\mathbf{i}}$ molar liquid flowrate per unit area based on the inner cross-sectional area of rotating bed $\left(\mathrm{lb}-\mathrm{mol} / \mathrm{ft}^{2} \cdot \mathrm{h}\right)$

Lo molar liquid flowrate per unit area based on the outer cross-sectional area of rotating bed $\left(\mathrm{lb}-\mathrm{mol} / \mathrm{ft}^{2} \cdot \mathrm{h}\right)$

m slope of equilibrium curve

N solute transferred ( $\mathrm{lb}-\mathrm{mol} / \mathrm{h}$ )

NTU number of transfer units

p partial pressure of solute (atm)

$P^{\prime} \quad$ partial pressure of solute at gas-liquid interface ( atm )

$\mathrm{p}^{*} \quad$ partial pressure of solute which is in equilibrium with liquid ( atm )

$P_{T} \quad$ total pressure ( atm )

$\mathbf{r}_{\mathbf{i}} \quad$ rotor inside radius $(\mathrm{ft}$ ) 
ro rotor outside radius ( $f t$ )

$\times \quad \mathrm{SO}_{2}$ mol fraction in liquid-phase

$x^{\prime}$ mol fraction of solute in the liquid-phase at interface

$x^{*} \mathrm{SO}_{2}$ mol fraction in the liquid-phase in equilibrium with the gas-phase

$x_{i} \quad \mathrm{SO}_{2}$ mol fraction in the rotor irilet

$x_{0} \mathrm{SO}_{2}$ mol fraction in the rotor outlet

y $\mathrm{SO}_{2} \mathrm{~mol}$ fraction in gas-phase

$y^{\prime} \quad$ mol fraction of solute in the gas-phase at interface

$y^{*} \quad \mathrm{SO}_{2}$ mol fraction in the gas-phase in equilibrium with the liquid-phase

$y_{\mathrm{i}} \quad \mathrm{SO}_{2}$ mol fraction in the rotor inlet

yo $\mathrm{SO}_{2}$ mol fraction in the rotor outlet

$Z$ depth of packing ( $\mathrm{ft}$ )

Greek Letters:

$\varepsilon \quad$ packing void fraction

$\Gamma \quad$ liquid flowrate per unit perimeter in wetted-wall colunm $(\mathrm{lb} / \mathrm{h} \cdot \mathrm{ft})$

H liquid viscosity $(\mathrm{lb} / \mathrm{h} \cdot \mathrm{ft})$

p liquid density ( $\left.\mathrm{lb}-\mathrm{mol} / \mathrm{ft}^{3}\right)$ or $\left(\mathrm{lb} / \mathrm{ft}^{3}\right)$

Dimensionless Groups:

Gr Grashof number $=g d^{3} p^{2} / \mu^{2}$ 
Reynols numbers: $d \mathrm{~L} / \mu$

Schmidt number $=\mu / \rho D$ 


\section{REFERENCES:}

1. Vivian, J. E., and D. W. Peaceman, A.IChE. J., 2, 437, (1956)

2 Davidson, J. F., Trans. Inst. Chem. Eng. (London), 37, 51 (1957)

3. Onda, K, E Sada, and Y. Murase, A.I.Ch.E. J., 5, 235 (1959)

4. Vivian, J.E, P.LT. Brian, and V. J. Kundonis, "The influence of gravitational Force on Gas absorption in a Packed Column", A.I.Ch.E J., 1088 (1965)

5. Ramshaw, C., "HIGEE Distillation-An Example of Proces Intensification", The Chemical Engineer, p. 13-14, Feb 1983

6. Ramshaw, C, and RH Mallinson, "Mass Transfer Process", U.S. Patent $4,283,255$ (1981)

7. Munjal S., M.P. Dudukovic, and P. Ramachandran, "Mass Transfer in Rotating Packed Beds Part 1: Development of Gas-Liquid-Solid Mass Transfer Correlations", Submitted for publication to Chemical Engineering Science (1987)

8. Munjal ., M.P. Dudukovic, and P. Ramachandran, "Mass-Transfer in a Rotating Packed-Bed with Countercurrent Gas-Liquid Flow", Presented at 77th Annual A.Ch.E. Meeting, Chicago.Illinois, November 10-15 (1985)

9. Munjal S., M. P. Dudukovic and P. Ramachandran, "Liquid Flow and Mass Transfer in Rotating Packed-Beds", presented at 2nd Yugoslav Chemical Engineers Congress, Dubrovnic, Yugoslavia, May 10-15 (1987)

10. Munjal S., MP. Dudukovic, and P. Ramachandran, "Mass-Transfer in Rotating Beds. Part 2: Experimental Results and Comparison with Theory 
52

and Gravity Flow", Submitted for publication to Chemical Engineering

Science", (1989)

11. Keyvani M., "Operating Characteristic of Rotating Beds", PhD. Thesis,

Case Western Reserve University, Cleveland, Ohio (1989)

12. Whitman, W. G., Chem. Met. Eng., 29, 4, 146, (1948)

13. Whitney, R P. and Vivian J. E., Chemical Engineering Progress, vol 45. No 5,p. 323 (1949)

14. Pearson D. et al., Chemical Engineering Progress, vol 47, no 5, p. 257 (1951) 


\section{APPENDD:}

TABLE 1

$\mathrm{SO}_{2}$-Air-Water $\quad \mathrm{G}=40 \mathrm{acfm} * \mathrm{y}_{\mathrm{i}}=1000 \mathrm{ppm} *$

$\begin{array}{lllllll}\# \mathrm{~g} & \mathrm{~L}=3 \mathrm{gpm}^{*} & \mathrm{~L}=3 & \mathrm{~L}=4 & \mathrm{~L}=4 & \mathrm{~L}=5 & \mathrm{~L}=5 \\ 12.41 & 144.15 & 160.17 & 111.86 & 114.11 & 106.58 & 99.79 \\ 97.36 & 210 & 295 & 144.77 & 150.86 & 134.42 & 118.71 \\ 143.56 & 530.43 & 384.41 & - & 167.81 & : 146.29 & - \\ 198.70 & - & 556.32 & - & 182.12 & 157.4 & - \\ 335.8 & - & - & 179.27 & 210.11 & 174.51 & 139.79 \\ 447 & - & - & - & 22282 & 186.37 & -\end{array}$

TABLE 2

$\mathrm{SO}_{2}$-Air-Water $G=20 \mathrm{acfm} \quad \mathrm{y}_{i}=1000 \mathrm{ppm}$

$\begin{array}{lcccc}\# \mathrm{~g} & \mathrm{~L}=2 & \mathrm{~L}=3 & \mathrm{~L}=4 & \mathrm{~L}=5 \\ 12.41 & 77.3 & 58.43 & 56.83 & 58.83 \\ 97.36 & 111.54 & 71.23 & 65.65 & 66.24 \\ 143.56 & 114.92 & 75.32 & 68.23 & 68.25 \\ 198.7 & 120.34 & 76.64 & 69.02 & 69.53 \\ 32.5 .8 & 12522 & 77.54 & 71.49 & 69.97 \\ 447 & 128.33 & 78.95 & 7236 & 70.87\end{array}$


TABLE 3

$\mathrm{SO}_{2}$-Air-Lime $\mathrm{G}=40$ acfm $y_{i}=1000 \mathrm{ppm}$

\begin{tabular}{lllll} 
\#g & $L=1$ & $L=1$ & $L=2 \cdot$ & $L=2$ \\
\hline 124 & 65.87 & 67.57 & 67.04 & 66.59 \\
31.79 & 71.22 & 71.22 & - & 73.62 \\
49.67 & 79.49 & 79.19 & 77.19 & 76.39 \\
97.36 & 87.05 & 86.33 & 79.29 \\
143.56 & 90.87 & 90.87 & 82.16 & 82.27 \\
198.7 & 92.27 & 92.10 & 84.92 & 85.41 \\
335.8 & 92.52 & 92.52 & 88.67 & 87.27 \\
447 & 93.80 & 92.94 & 91.11 & 89.64
\end{tabular}

TABLE 4

$\mathrm{SO}_{2}$-Air-Lime $\quad G=55 \mathrm{acfm} \quad \mathrm{y}_{\mathrm{i}}=1000 \mathrm{ppm}$

\begin{tabular}{lcccc} 
\#g & $\mathrm{L}=1$ & $\mathrm{~L}=1$ & $\mathrm{~L}=2$ & $\mathrm{~L}=2$ \\
\hline 12.41 & 82.41 & 89.06 & - & 69.29 \\
27.94 & - & - & - & 74.84 \\
31.79 & 94.02 & 92.11 & - & - \\
49.67 & 95.67 & 98.08 & 72.74 & - \\
97.36 & 111.2 & 105.63 & - & - \\
143.56 & 115.44 & 109.86 & - & 81.24 \\
198.7 & 118.48 & 113.99 & 78.86 & 85.55 \\
263.69 & 119.53 & 115.94 & - & - \\
335.8 & 121.14 & 117.45 & - & - \\
447 & 121.7 & 118.48 & 86.88 & 93.22
\end{tabular}


- acfmi actual cubic feet per minute ppm: part per million by volume

gpm: gallon per minute 

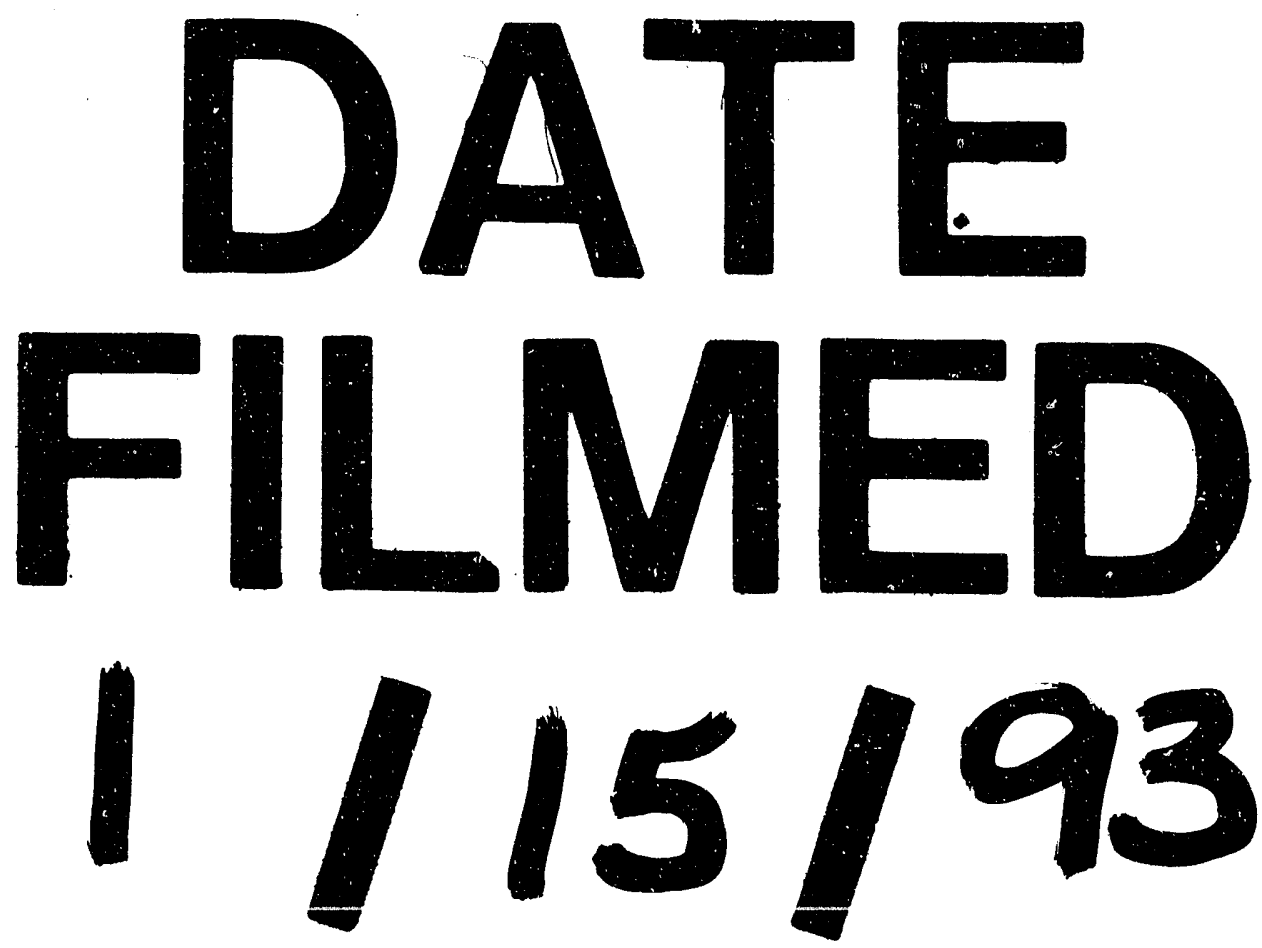
